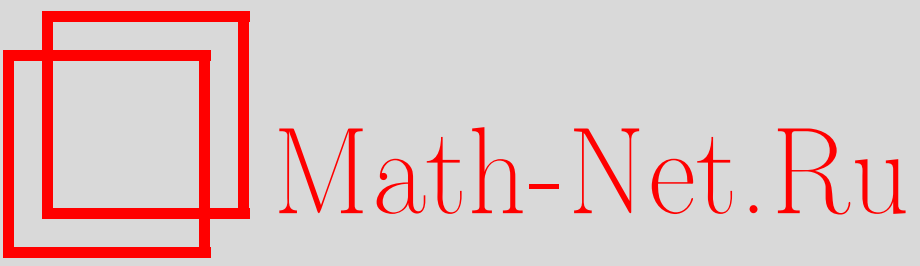

Е. В. Щепин, Арифметика теории размерности, УМH, 1998, том 53, выпуск 5, 115-212

DOI: https://doi.org/10.4213/rm72

Использование Общероссийского математического портала Math-Net.Ru подразумевает, что вы прочитали и согласны с пользовательским соглашением

http://www . mathnet.ru/rus/agreement

Параметры загрузки:

IP : 3.82 .47 .9

26 апреля 2023 г., 12:38:17 


\title{
АРИФМЕТИКА ТЕОРИИ РАЗМЕРНОСТИ
}

\author{
Е. В. ЩЕпин
}

\section{СОДЕРЖАНИЕ}

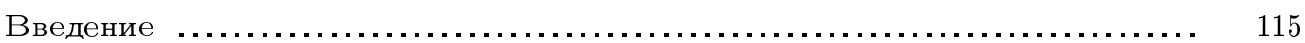

Глава 1. Алгебра .................................................... 134

$\S 1$. Тензорная классификация абелевых групп ....................... 134

$\S 2$. Кюннетовские алгебры полигрупп ............................ 143

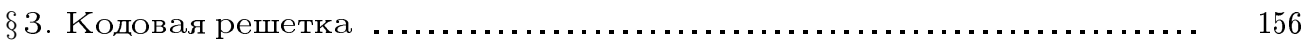

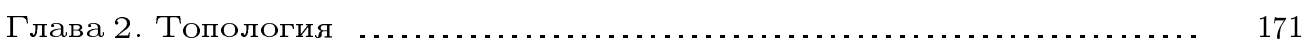

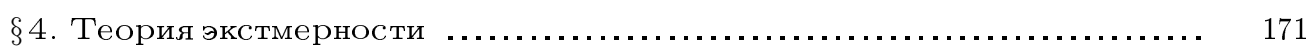

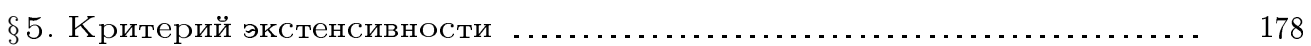

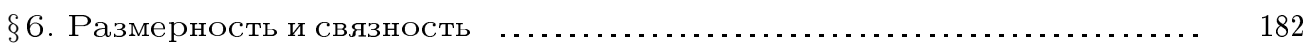

Глава 3. Геометрия ….......................................... 188

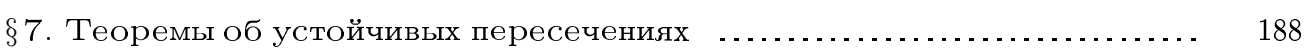

$\S 8$. Верхние оценки размерности пересечения $\ldots \ldots \ldots \ldots \ldots \ldots \ldots \ldots \ldots \ldots . . . \ldots \ldots$

$\S 9$. Нижние оценки размерности пересечения

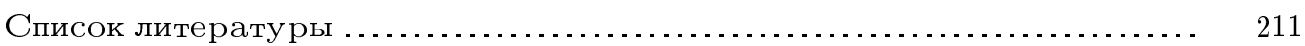

\section{Введение}

Три великих математика, А. Лебег, А. Пуанкаре и Г. Кантор, в начале века высказали идеи, легшие в основу теории размерности. А. Лебег дал определение размерности с помошью покрытий. А. Пуанкаре дал ей индуктивное определение. Г. Кантор определил кривую как нигде не плотный континуум.

Теорию размерности в первой половине столетия назьвали теорией размерности Брауэра-Урысона-Менгера. Л. Брауэр, доказав негомеоморфность различных евклидовых пространств - теорему об инвариантности числа измерений, показал принципиальную возможность построения топологической теории размерности. Построили же теорию размерности П. С. Урысон и К. Менгер.

Работа выполнена при поддержке Российского фонда фундаментальных исследований (грант № 96-01-01166a). 
Вопрос, приведший Урысона к созданию теории размерности, был поставлен перед ним его учителем Д. $\Phi$. Егоровым [1] и заключался в том, чтобы дать адекватное общее определение поверхности, т.е. множества двух измерений.

Тут читатель может спросить: а как же с определениями размерности Лебега и Пуанкаре? Разве они не были известны Егорову и Урысону? Тут мы сталкиваемся с двойным недопониманием. Во-первых, под определением размерности, как его понимали Лебег, Пуанкаре и Брауэр, имелось в виду определение размерности евклидова пространства, т.е. указание способов топологического определения геометрического числа измерений пространства. Конечно, всякое такое определение можно пытаться распространять на более или менее произвольные множества, но до Урысона этого никто не делал. Во-вторых, задача дать адекватное определение заключалась не столько в том, чтобы дать определение, сколько в том, чтобы доказать его адекватность. То есть продемонстрировать, что данное определение соответствует нашим интуитивным представлениям.

Если в отношении определения Лебега была ясность, как его распространить на любые множества, то в отношении определения Пуанкаре таковой не было. Проблема здесь заключалась уже в том, чтобы правильно определить значение термина "разбивает”. Урысон сумел это сделать, в то время как Л. Брауэр придал этому понятию такой смысл, на основе которого автор не берется доказать, что индуктивное определение Пуанкаре правильно определяет размерность евклидова пространства. Брауэр предлагал называть множество $A$ разбивающим пространство $X$ между точками $a$ и $b$, если всякий континуум в $X$, содержаший $a$ и $b$, пересекает $A$. Это определение двойственно определению, взятому на вооружение Урысоном, которое можно сформулировать так: дополнение к $A$ ретрагируется на двухточечное множество. Определение Брауэра “гомологично", определение Урысона "когомологично”. Здесь мы в простейшей форме сталкиваемся с преимушеством когомологий в теории размерности над гомологиями.

Важнейшим аргументом Урысона в доказательстве адекватности данного им общего определения кривой, поверхности и $k$-мерного многообразия является то, что определения размерности Лебега и Пуанкаре для произвольных множеств евклидова пространства дают одинаковый результат.

Современное доказательство адекватности определения топологической размерности могло бы заключаться в предъявлении ее аксиоматики. Она весьма проста и наглядна.

АКСИОМАТИКА АЛЕКСАНдРовА. ${ }^{1}$ Существует единственная функиия, сопоставляющая каждому непустому конечномерному компакту $X$ челое число $d(X)$ и удовлетворяющая следующим условиям:

(1) (аксиома инвариантности) если $X$ гомеоморфно $Y, \operatorname{mo~} d(X)=d(Y)$;

(2) (аксиома нормировки) $d\left(I^{n}\right)=n$ для $n$-мерного куба;

(3) (аксиома суммы) $d(X \cup Y)=\max \{d(X), d(Y)\}$;

(4) (аксиома границы) $d(\partial U)<d(X)$ для некоторого открытого $U \subset X$;

(5) (аксиома непрерьвности) $d\left(\lim X_{k}\right) \leqslant \lim d\left(X_{k}\right)$.

\footnotetext{
${ }^{1}$ Приведенная аксиоматика является небольшой модификацией оригиналшной аксиоматики П. С. Александрова из книги [2]. Так, аксиома непрерывности выступает в ней вместо аксиомы Брауэра.
} 
Определение размерности через покрытия, данное Лебегом, давно утвердилось в математике, как основное. Лебегова размерность является сейчас синонимом понятия топологическая размерность. Что же главное из того, что сумел доказать Урысон про лебегову размерность? Как сейчас представляется, главное - это то, что Урысон научился строить перегородки размерностей меньших, чем объемлюшее пространство. И сильнейшей формулировкой его достижений является следующая теорема о разложснии:

Теорема УРЫСОна. Если $\operatorname{dim} X=n$, mо для любого $k \leqslant n$ найдется такое $X^{\prime} \subset X$, чmo $\operatorname{dim} X^{\prime}=k u \operatorname{dim}\left(X \backslash X^{\prime}\right)=n-k-1$.

Эту теорему органически дополняет следующий результат. ${ }^{2}$

Hepabehство Урысона. $\operatorname{dim} X \cup Y \leqslant \operatorname{dim} X+\operatorname{dim} Y+1$.

Пространства, фигурирующие в этих теоремах, не предполагаются компактными, а множества замкнутыми. Именно поэтому урысоновская формула объединения отличается от аксиомы суммы. Сам Урысон считал неравенство объединения второстепенным результатом. Дело в том, что основным объектом его исследований выступают замкнутые множества евклидова пространства, а изучением незамкнутых множеств он занимался лишш постольку, поскольку это было необходимо для изучения замкнутых. Эта статья следует тому же принципу.

Гомологическая теория размерности. Эстафету развития теории размерности от П. С. Урысона принял П. С. Александров [4], инициировавший гомологический подход к теории размерности.

После Урысона главной проблемой теории размерности стало получение положи тельной, как пишет Л.С. Понтрягин (см. [5]), характеризации размерности. Теория Урысона страдала односторонностью, которая проявлялась в том, что она позволяла оценивать размерность пространств сверху, но была весьма неэффективна для оценок снизу.

Конкретная задача, которую в конце двадшатых - начале тридшатых годов пытались решить П. С. Александров и Л. С. Понтрягин, заключалась в том, чтобы найти в $n$-мерном компакте какой-нибудь негомологичньй нулю относительньй $n$-мерньй цикл. То есть доказать совпадение урысоновской размерности с гомологической, которая определялась как наибольшая размерность из имеюшихся в пространстве ненулевых относительных циклов.

Если какое-то подмножество в $\mathbb{R}^{n}$ имеет размерность $\geqslant k$, то оно, с одной стороны, не допускает малых сдвигов в $k$-мерные остовы мелких триангуляций пространства, а с другой, малыми сдвигами не снимается с $(n-k-1)$-мерных остовов мелких триангуляций. П. С. Александров и Л. С. Понтрягин в поисках цикла опирались на эти двойственные свойства размерности.

Л.С. Понтрягин, развивший двойственность Александера так, что она стала применимой к компактам, руководствовался невозможностью снятия с остовов дополнительной размерности. На этом пути [5] ему надо было найти цикл в дополнении ком-

\footnotetext{
2 Это неравенство в книге [2] названо неравенством Урысона-Менгера, но автор предпочитает для него сохранять название неравенство Урысона, которое было общепринято до появления книги [2]. История вопроса по этому поводу приведена в предисловии к книге [3] и в статье С.А. Богатого, содержащейся в этом же номере журнала.
} 
пакта, который локально был бы зацеплен с компактом. При этом ему было известно, что сфероид (элемент гомотопической групш), локально зацепленньй с компактом, сушествует. Для того чтобы это сделать, как мы знаем теперь, ему достаточно было, перевложив компакт, ликвидировать фундаментальную групу у дополнения и доказать теорему Гуревича об изоморфизме первой нетривиальной групшы гомотопий и гомологий. Как мы видим, программа весьма серьезная, учитывая то, что и групшы гомотопий в то время еще не были определены. Л.С. Понтрягин не справился с ней. Он выбрал ложный путь. Ему показалось, что сначала надо разобраться с гомотопической классификацией отображений сфер разных размерностей. В этой задаче он достиг выдающихся успехов, изобрел характеристические классы и многое другое.

В то же время П. С. Александрову [4], опиравшемуся на данную им же характеристику размерности посредством сдвигов в полиэдры, фактически потребовалось искать нетривиальньй коцикл (когомологий тогда тоже еще не было), в чем ему помог Хопф, доказавший по его просьбе знаменитую теорему Хопфа об отображениях $n$-мерного полиэдра в $n$-мерную сферу.

П. Урысон развил внутренний подход к определению размерности. Внешнее определение размерности заключается в определении размерности компакта через свойства его расположения в евклидовом пространстве. Так, внешним является определение канторовской кривой, столь впечатлившее П. С. Урысона, что основное его произведение по теории размерности [1] названо им "Мемуаром о канторовых многообразиях".

После того как внутреннее построение теории размерности было завершено, естественно встал вопрос о внешнем ее определении. То есть об определении размерности компакта через свойства его расположения в объемлющем пространстве и, в частности, через свойства его дополнения.

Проблема гомологической характеризации размерности и проблема ее внешнего определения были, по существу, разными формами одного и того же вопроса. Их эквивалентность вытекала из двойственности Александера-Понтрягина, согласно которой гомологии замкнутого множества двойственны гомологиям его дополнения. Поэтому нахождение цикла внутри компакта влекло существование локально зацепленного с ним цикла дополнительной размерности в его дополнении, а существование зацепленного цикла в свою очередь позволяло найти относительный цикл дополнительной размерности в компакте.

Таким образом, следуюшая теорема о зацеплениях является одной из форм ответа на рассмотренные вопросы.

ТЕОРЕМА АЛЕКСАНДРОВА. Замкнутое множество странства имеет размерность $k$ в том и только том случае, когда в дополнении к нему имеются локально зацепленные с ним $(n-k-1)$-мерные цикльи $и$ никакой иикл меньшей размерности нигде с ним не зачеплен.

Проблема размерности произведения. Интрига последующего развития теории размерности концентрируется вокруг проблемь размерности произведения. То, что натуральные числа делятся на простые и составные, не имело на первом этапе развития теории размерности никакого значения. Однако, как оказалось, это обстоятельство невозможно игнорировать, пытаясь научиться определять размерность произведения компактов. 
Так, из результатов Урысона легко получалось неравенство $\operatorname{dim} X \times Y \leqslant$ $\operatorname{dim} X+\operatorname{dim} Y$. Но уже тот факт, что при умножении на отрезок размерность увеличивается, не был доказан Урысоном и остается за пределами монографий, излагаюших теоретико-множественную теорию размерности. Этот факт был доказан П.С. Александровым [4] как одно из первых приложений гомологической теории размерности.

В первом приближении проблема размерности произведения понималась как доказательство логарифмического закона сложения размерностей, согласно которому размерность произведения равна сумме размерностей сомножителей. Намерение доказать этот закон сушествовало с самого начала теории размерности.

Эта проблема оказалось теснейшим образом связанной со следуюшей проблемой коэффичиентов. В определении гомологической размерности участвует група коэффициентов. Так, П. С. Александров предпочитал пользоваться гомологиями по модулю 2, чтобы "не путаться со знаками". И в первом приближении проблема коэффициентов заключалась в том, чтобы доказать, что определение гомологической размерности не зависит от групшы коэффициентов.

Обе эти проблемы были решены одновременно Л. С. Понтрягиным в 1930 году [6], которьй построил свои понтрягинские поверхности. Но Л. С. Понтрягин в этой работе не только опроверг логарифмический закон для топологической размерности, но и доказал его справедливость для размерностей по модулю простого числа.

Нарушение логарифмического закона делает осмысленным введение понятия размерностного типа компакта (впервые введенного только в 1994 году [7]). Будем говорить, что два компакта $X$ и $Y$ имеют одинаковый размерностный тип, если $\operatorname{dim} X \times Z=\operatorname{dim} Y \times Z$ для любого компакта $Z$. Размерностньй тип является более тонкой характеристикой, чем размерность, и обозначается DIM $X$. Множество размерностных типов естественным образом упорядочено, а именно, считаем, что $\operatorname{DIM} X \geqslant \operatorname{DIM} Y$, если $\operatorname{dim} X \times Z \geqslant \operatorname{dim} Y \times Z$ для всякого $Z$. И операция произведения компактов индуцирует операцию на множестве типов. Эту операцию мы будем обозначать знаком [+]. Таким образом, $\operatorname{DIM}(X \times Y)=\operatorname{DIM} X[+] \operatorname{DIM} Y$. Множество размерностных типов с описанными отношением частичного порядка и операцией будем называть размерностной алгеброй и обозначать $\mathfrak{D M}$. Ясно, что полное решение проблемы произведения должно включать в себя как критерий совпадения типов, так и описание размерностной алгебры.

С другой стороны, возможность несовпадения размерностей по различным группам коэффициентов делает осмысленным введение понятие (ко) гомологического размерностного типа. А именно, два компакта $X$ и $Y$ назьваются имеюшими одинаковьй (ко) гомологический размерностныи й тип, если $\operatorname{dim}_{G} X=\operatorname{dim}_{G} Y$ для любой абелевой групшы $G$.

После М.Ф. Бокштейна и А.Н. Дранишникова мы знаем, что понятие когомологического размерностного типа для конечномерных компактов оказалось тождественным понятию размерностного типа. А основные вопросы, которые возникли после работы Понтрягина и решение которых имело большое значение для полного решения проблемы произведения, были таковы: 
ПРОБЛЕМА РАЗМЕРНОЙ ПОЛНОЦЕННОСТИ. Охарактеризовать размерно-полноценные компакты $X$, т.е. такие, для которых равенство $\operatorname{dim} X \times Y=$ $\operatorname{dim} X+\operatorname{dim} Y$ выполнено при любом $Y$.

ПРОБЛЕМА КОЭФФИЦИЕНТНОГО БАЗИСА. Найти минимальный набор абелевых групп, знания размерностей по которьм достаточно, чтобь определить размерность компакта по любой группе.

Обе эти проблемы ставились П.С. Александровым, причем в постановке первой принимал участие А.Н. Колмогоров. После работы Понтрягина естественно было бы ожидать, что в качестве коэффициентного базиса выступают аддитивные группы полей (поля одинаковой характеристики дают одинаковые размерности). В таком случае топологическая размерность компакта определялась бы как максимум из его “полевых" размерностей. А равенство типов DIM $X=\operatorname{DIM} Y$ естественно было ожидать равносильным совпадению размерностей $\operatorname{dim}_{p} X=\operatorname{dim}_{p} Y$ для любого простого $p \geqslant 0$, здесь для компакта $X$ и числа $p$, простого или нуля, через $\operatorname{dim}_{p} X$ обозначается размерность по модулю $p$, а $\operatorname{dim}_{0} X$-это рациональная размерность, т.е. размерность с рациональными коэффициентами.

Этим ожиданиям, однако, не суждено было сбыться. В 1949 году В.Г. Болтянский, построив пример двумерного компакта с трехмерных квадратом [8], опроверг эти представления. Пример Болтянского имеет все полевые размерности равными единице. Построенньй пример позволил Болтянскому также и решить проблему характеризации размерно-полноценных компактов [9]. Оказалось, что размерная полноценность компактов характеризуется размерностями с коэффициентами в көазициклических әруппах $\mathbb{Q}_{p}$.

Теорема БолтянСКОГо. Компакт $X$ размерно-полноценен в том и только том случае, когда $\operatorname{dim}_{\mathbb{Q}_{p}} X=\operatorname{dim} X$ для любого простого $p$.

Из этой теоремы видно, что проблема коэффициентов и логарифмический закон были, по сушеству, разными формами одной и той же проблемы.

Проблема коэффициентного базиса была решена М. $\Phi$. Бокштейном $([10],[11])$. Оказалось, что для получения полной системь групп нужно к полям $\mathbb{Q}, \mathbb{Z}_{p}$ и квазициклическим групам $\mathbb{Q}_{p}$ добавить серию аддитивных груп локальных колец $\mathbb{Z}_{(p)}$. Кольцо $\mathbb{Z}_{(p)}$ является подкольцом поля $\mathbb{Q}$ рациональных чисел, образованным дробями $m / n$, со знаменателем взаимно простым с $p$. Решение этой проблемы позволило Бокштейну заодно решить и проблему определения размерности произведения. Первая Теорема Бокштейна предъявила счетное множество групп - систему Бокитейна, знание размерностей по которым позволяет эффективно, по алгоритму Бокитейна, определить размерность по любой группе коэффициентов. В частности, она позволяла определить и топологическую размерность, ибо последняя, согласно Александрову, также является гомологической. Помимо системы и алгоритма эта теорема Бокштейна, включает в себя еще и неравенства Бокштейна, которые представляют соотношения между размерностями по группам системы Бокштейна верные для любых компактных пространств.

Вторая Теорема Бокштейна показьвает, как ведут себя размерности по базисным группам при умножении. То есть она позволяет определить гомологические размер- 
ности произведения, а значит, и его топологическую размерность, через гомологические размерности сомножителей.

Тут мы оговоримся. Начиная с упомянутых работ Бокштейна когомологии вытесняют гомологии из теории размерности. И то, что называется теперь гомологической теорией размерности, правильнее было бы назьвать когомологической.

Проблема реализации. Таким образом, Вторая Теорема Бокштейна позволила доказать, что совпадение когомологического типа компактов влечет совпадение их размерностного типа. Верно ли обратное? Ответ на этот вопрос зависел от решения следуюшей проблемы реализачии, которая после результатов Бокштейна надолго стала одной из основных проблем теории размерности.

ПРОБЛЕМА БОЛТЯНСКОГО-БОКШТЕЙНА. Всякая ли комбинация размерностей по базисньм группам, удовлетворяющая неравенствам Бокштейна, является совокупностью размерностей некоторого компакта?

Важные результаты по реализации были получены Ю. Кодамой [12], [13] и В. И. Кузьминовым [14]. Кодама, в частности, построил первый пример компакта, у которого рациональная размерность отличалась от всех размерностей по простому модулю. Он также продублировал конструкцию Болтянского и ввел в употребление понятие пробного компакта. Пробные компакты Кодама тестировали размерную полноценность.

Кузьминов же проанализировал алгебру Бокштейна - алгебру размерных функций, которые группам системы Бокштейна ставили в соответствие натуральные числа так, чтобы вьполнялись неравенства Бокштейна, а “складывались” размерные функции по правилам, согласно которым определялись размерности произведения компактов. Кузьминов выделил в алгебре Бокштейна базис- основные функи,ии, через которые посредством операций взятия верхней грани и сложения получались все остальные. Задача реализации была таким образом редуцирована им к проблеме построения четырех серий основных компактов, реализуюших базисные размерные функции. Сам Кузьминов построил все базисные компакты размерности 3. Это позволило ему сушественно обобшить понятие пробного компакта и получить результат, в некоторой степени сводяший гомологическую теорию размерности к теоретико-множественной.

Теорема КУЗьмИновА. Для всякой абелевой группь $G$ и любого натурального $n$ существует такой компакт $T_{G}^{n}$, что для любого компакта $X$ размерности $\operatorname{dim} X \leqslant n$ имеет место равенство $\operatorname{dim}_{G} X=\operatorname{dim} X \times T_{G}^{n}-\operatorname{dim} X$.

Лишь десять лет назад А.Н. Дранишников [15] построил компакты, реализуюшие все комбинации когомологических размерностей, удовлетворяющих неравенствам Бокштейна, и тем самьм поставил точку в проблеме размерности произведения компактов. Таким образом, размерностная алгебра оказалась изоморфной алгебре Бокштейна. Реализационная теорема Дранишникова логически завершает второй - гомологический - этап в развитии теории размерности. Помимо проблемы Болтянского-Бокштейна им были решены другие важные реализационные проблемы. Это проблема Александрова о сушествовании бесконечномерного компакта конечной когомологической размерности и проблема Борсука о существовании размерно неполноценных локально стягиваемых компактов. Итог этому периоду подведен в обзорной статье [16]. 
Гомологическая теория, столь эффективная для определения размерностей произведения, оказалась беспомощной перед задачей о доказательстве гомологических аналогов неравенства Урысона. А вопрос о существовании перегородок, гомологическая размерность которых была бы меньше, чем гомологическая размерность исходного пространства, даже не поднимался. Причина последнего в том, что вопрос имел очевидньй отрицательньй ответ: если гомологическая размерность компакта равна единице, а топологическая больше единицы, то компакт нельзя разбить множеством гомологической размерности 0 , потому что гомологическая размерность 0 влечет топологическую размерность 0 . Однако для случая, когда гомологическая размерность пространства больше 1, ответ на этот вопрос, как мы увидим, положителен. Таким образом, гомологический подход к размерности оказался слаб как раз в том, в чем был так силен теоретико-множественньй, и наоборот, силен в проблеме произведения и внешней характеризации, где последний так слаб.

Проблема неустойчивого пересечения. Десять лет назад возникла проблема, изменившая весь облик теории размерности. Сейчас, когда очертания новой теории определились, можно ее квалифицировать как третий - гомотопический - этап развития теории размерности. Первьй теоретико-множественный этап имел дело в основном с внутренним строением компактов, не знал алгебры и породил формулу объединения. Второй, гомологический, основные достижения имел в алгебре и внешней характеризации размерности и породил формулу произведения. На третьем этапе, как мы увидим, в основе выступает взаимодействие внутреннего с внешним, а основная формула этого этапа - формула пересечения - синтетична по отношению к формулам объединения и произведения.

Началось с предпринятой $\Phi$. Анщелем попытки обратить теорему Нёбелинга-Понтрягина, которая утверждает, что всякое непрерывное отображение $n$-мерного компакта в $\mathbb{R}^{2 n+1}$ аппроксимируется вложениями. Вопрос Анцеля гласил: если всякое отображение компакта в $\mathbb{R}^{2 n}$ аппроксимируется вложениями, то можно ли утверждать, что размерность компакта меньше чем $n$ ? Или, другими словами, верно ли, что всякий компакт размерности $n$ можно отобразить в $\mathbb{R}^{2 n}$ с устойчивыми самопересечениями. Для полиэдров это верно. В работе 1989 года [17] Д. Мак-Каллох и Л. Рубин дали на этот вопрос положительный ответ и ошиблись. Это дало старт серии работ различных авторов, из которых выделим С. Спеша (см. [18]), приведших уже через год к полному решению этого вопроса. Оказалось, что всякое отображение компакта $X$ в евклидово пространство $\mathbb{R}^{2 n}$ аппроксимируется вложениями в том и только том случае, когда $\operatorname{dim} X^{2}<2 n$. А так как существуют компакты, у которых размерность квадрата меньше удвоенной размерности самого компакта (компакты В.Г. Болтянского [8]), то и ответ на вопрос Анцеля оказьвается отрицательньм. То есть эффект странного поведения компактов, наблюдающийся в этой задаче, оказался обусловлен все тем же нарушением логарифмического закона. Следуюшим естественньм вопросом оказался такой:

Верно ли, что компакты $X, Y$, имеющие размерность произведения меньше чем $n$, не имеют в $\mathbb{R}^{n}$ устойчивых пересечений?

Проблема эта последовательно решалась путем ослабления размерностных ограничений на компакты $X$ и $Y$ в соперничестве польской и русской школ (см. [18], [19]). Сильнейший на данный момент результат по этой проблеме принадлежит А.Н. Дра- 
нишникову [20], которьй получил положительное решение для компактов коразмерности 3. Для коразмерности 2 вопрос все еше открыт. Решение этой проблемы и потребовало от Дранишникова развития гомотопического подхода к теории размерности.

Отношение экстенсивности. На третьем этапе основную роль в теории размерности стали играть гомотопические методы. В фокус интересов попало введенное К. Куратовским отношение экстенсивности. Пространство $X$ назьвается әкстенсивным по отношению к пространству $Y$, пишем $X \tau Y$, если всякое непрерывное отображение любого замкнутого подпространства $X^{\prime} \subset X$ в $Y$ продолжается до непрерьвного отображения всего $X$. При этом пространство $Y$ называется коэкстенсивным пространству $X$. Это отношение позволяет охарактеризовать как топологическую, так и когомологические размерности. Так, теорема Александрова в форме Гуревича гласит: $\operatorname{dim} X \leqslant n$, если и только если $X \tau S^{n}$. А экстенсивность $X$ по отношению к комплексу Эйленберга-Маклейна $K(G, n)$ равносильна неравенству $\operatorname{dim}_{G} X \leqslant n$.

Гомотопические аналоги известных ранее теорем теории размерности оказались имеющими доказательства столь же элементарные, как их теоретико-множественные аналоги, и даюшими результаты более сильные, чем их гомологические аналоги. Причем во многих случаях гомологические результаты были впервые получены именно гомотопическим путем.

В основе гомотопического подхода к теории размерности лежит следующий критерий экстенсивности:

ТЕОРЕМа ДРАНИШНИКОВА [21]. Следующие условия равносильны для конечномерного компакта $X$ и односвязного комплекса $K$ :

(1) $X \tau K$;

(2) $\operatorname{dim}_{H_{k}(K)} X \leqslant k$ при любом $k \geqslant 1$;

(3) $\operatorname{dim}_{\pi_{k}(K)} X \leqslant k$ при любом $k \geqslant 1$

(через $H_{k}(K)$ обозначены приведенные целочисленные гомологии комплекса $K$, а через $\pi_{k}(K)$ - гомотопические группы).

Доказательство критерия экстенсивности опирается на такие достижения гомотопической топологии, как теория локализаций и пополнений Сулливана и теорема Дольда-Тома о бесконечной симметрической степени.

Критерий экстенсивности осуществляет связь между гомотопическим и гомологическим подходами к теории размерности. Он сконщентрировал в себе все неэлементарное, что есть в гомотопическом подходе.

Отметим, что из критерия экстенсивности вытекает такое следствие.

СлЕДСТВИЕ. При $n>1$ для компакта $X$ әкстенсивность по отношению $\kappa$ комплексу Эйленберга-Маклейна $X \tau K(G, n)$ равносильна әкстенсивности по отношению к комплексу Мура $X \tau M(G, n)$.

Действительно, это непосредственно вытекает из критерия экстенсивности Дранишникова, ведь гомологии комплекса Мура как раз совпадают с гомотопиями комплекса Эйленберга-Маклейна. Важным нерешенным вопросом (поставленным А.Н. Дранишниковьм) является вопрос о справедливости этого следствия для $n=1$. 
ПРОБЛЕМА НЕОДНОСВЯЗНОСТИ. Верно ли, что для любого компакта $X$ и любой счетной группь $G$ равносильны условия $X \tau M(G, 1)$ и $X \tau K(G, 1)$ ?

Экстмерность. Основные достижения третьего этапа теории размерности связаны с именем А.Н. Дранишникова, которьй и ввел в обиход термин extensional dimension для возникшей теории. Мы будем именовать ее теория әкстмерности. При этом под әкстмерностью компакта мы будем подразумевать класс гомотопических типов всех коэкстенсивных ему комплексов и обозначать ее $\mathfrak{D i m} X$. И договоримся использовать знак неравенства для экстмерностей вместо противоположного знака включения. То есть неравенство $\mathfrak{D i m} X \leqslant \mathfrak{D i m} Y$ равносильно включению $\mathfrak{D i m} Y \subset \mathfrak{D i m} X$, а неравенство $\mathfrak{D i m} X \leqslant K$ расшифровывается как включение $K \in \mathfrak{D i m} X$. Тогда неравенство $\operatorname{dim} X \leqslant n$ оказьвается равносильным такому: $\mathfrak{D i m} X \leqslant S^{n}$.

Решение вопроса о справедливости неравенства Урысона для когомологических размерностей является яркой иллюстрацией продуктивности новых идей. Сначала, с одной стороны, как применение теории пересечений компактов, был построен пример [22], опровергающий неравенство Урысона для некоторых груп. А с другой, Л. Рубин [23] доказал это неравенство для целых чисел. При этом в работе Рубина, впервые в контексте этой задачи, была применена конструкция джойна, сьгравшая в дальнейшем решаюшую роль. Полное решение этой проблемы, полученное Ю. Дыдаком [24], [25], может быть сформулировано так:

$$
\mathfrak{D i m}(X \cup Y) \leqslant \mathfrak{D i m} X * \mathfrak{D i m} Y \text {. }
$$

Здесь * используется для обозначения операции джойна. Доказательство Дыдака элементарно и умешается на полстраницы. Эта формула обобщает неравенство Урысона в силу вьшеупомянутой теоремы Александрова. В то же время эта теорема Урысона-Дыдака с помошью критерия экстенсивности Дранишникова позволяет доказать справедливость неравенства Урысона для когомологической размерности над любым кольцом.

Другая проблема когомологической теории размерности, долго не поддававшаяся решению и нашедшая решение в рамках теории экстмерности, - проблема пополнения. Теорема Ольшевского [26] гласит: для всякого сепарабельного метрического пространства $X$ найдется объемлющее его полное пространство $\widetilde{X}$ той же экстмерности.

Экстраординарные размерности. До появления теории экстмерности топологи даже не подозревали, что гомологические размерности почти удовлетворяют аксиоме гранищы. Проблемы о разбиении гомологии находились в центре внимания, но решались плохо (см. [27]). Лишш в 1966 году Е. Г. Скляренко [28] опубликовал безупречное доказательство теорем о разбиении гомологий и когомологий, которые давно предполагались П. С. Александровым. При этом условия на разбивающее множество формулировались в терминах топологической, а не гомологической размерности. Оказывается, что для любой группы $G$ всякий компакт $X$ размерности $\operatorname{dim}_{G} X>1$ имеет открытую базу топологии с границами $G$-размерности меньшей $\operatorname{dim}_{G} X$. Этот факт можно получить из следуюшей теоремы разложения Дранишникова, являюшейся экстмерным аналогом теоремы Урысона. 
Теорема УРЫСОНА-ДРАнИшнИковА. Если $\mathfrak{D i m} X \leqslant K * L$, mo $X$ представляется в виде облединения $X=X_{1} \cup X_{2}$ подпространств әкстмерностей $\mathfrak{D i m} X_{1} \leqslant$ $K u \mathfrak{D i m} X_{2} \leqslant L$.

Действительно, если $\operatorname{dim}_{G} X=n$, то $X \tau K(G, n)$, и потому $X \tau M(G, n)$. Но $M(G, n)=\Sigma M(G, n-1)=S^{0} * M(G, n-1)$. В силу теоремы о разложении получаем $X=X_{0} \cup X_{1}$, где $X_{0} \tau S^{0}$ и $X_{1} \tau M(G, n-1)$. Из второго условия вытекает $X_{1} \tau K(G, n-1)$, откуда $\operatorname{dim}_{G} X_{1} \leqslant n-1$. А из первого $-\operatorname{dim} X_{0}=0$. Пусть $U \subset X_{0}$ - открыто-замкнуто в $X_{0}$. Пусть $U^{\prime}$ - открытое подмножество в $X$, для которого $U^{\prime} \cap X_{0}=U$. Тогда граница $U^{\prime}$ лежит в $X_{1}$ и, следовательно, имеет $G$-размерность меньше $n$.

Доказанное утверждение позволяет интерпретировать когомологические размерности как әкстраординарные. По аналогии с теорией гомологий и когомологий, будем назьвать экстраординарной теорией размерности всякую размерностную функцию (функцию, сопоставляюшую компактам натуральные числа), удовлетворяющую всем аксиомам Александрова за исключением аксиомы нормировки. Тогда всякая абелева группа порождает экстраординарную теорию размерности $d_{G}$, определяемую по следуюшему правилу: $d_{G}(X)=\operatorname{dim}_{G} X-1$, если $\operatorname{dim}_{G} X \geqslant 1$, и $d_{G}(X)=0$, если $\operatorname{dim}_{G} X=0$. И всякий комплекс $K$ порождает экстраординарную теорию размерности по следуюшему правилу: $\operatorname{dim}_{K} X=n$, если $n$ - наименьшее число, для которого $X$ экстенсивен по отношению к $n$-кратной надстройке $\Sigma^{n} K$. Сам комплекс $K$ назьвается классифицирующим пространством для порожденной им экстраординарной теории размерности.

Теорию размерности с коэффициентами в неабелевой групе $G$ можно определить как экстраординарную теорию размерности, классифищируюшим пространством которой служит комплекс Эйленберга-Маклейна $K(G, 1)$.

Если $G$ совершенна, то $\operatorname{dim}_{G}$ принимает всего два значения, поскольку $\Sigma K(G, 1)$ стягиваемо. Теории размерности с некоммутативными коэффициентами посвяшены работы [30], [31]. В них, в частности, доказано, что компакт $X$, экстенсивньй по отношению к $K(G, 1)$ для любой совершенной группы $G$, является топологически двумерным.

Экстмерное решение проблемы реализации. Теория экстмерности позволила дать качественно иное решение проблемы реализации. Проблема неустойчивого пересечения была сведена в работе [7] к следуюшей проблеме промежуточных размерностей:

Верно ли, что $\mathbb{R}^{n}$ содержит компакты всех размерностных типов, размерности $\leqslant n-2$ ?

Решив эту проблему, Дранишников [20] заново решил проблему реализации. Данное им решение опиралось на экстмерную версию теоремы Эйленберга и двойственность Ситникова. Данное в $\S 6$ решение проблемы реализации еше проще. Оно опирается лишш на теоремы Урысона-Дранишникова и Урысона-Дыдака. При этом результат получается сильнее. Оказьвается, что проблема промежуточных размерностей имеет положительное решение, даже если $\mathbb{R}^{n}$ заменить на любой $n$-мерньй размерно-полноценньй компакт. 
Гомологическая теория связности. Двойственной к когомологической теории размерности выступает гомологическая теория связности. Такой теории не значится в рубриках реферативных журналов. В этой статье она упоминается впервые, хотя изоморфная ей теория содержится в статье Дранишникова-Дыдака [29]. И нет в литературе даже специального обозначения для того, что мы будем назьвать индексом связности комплекса.

Индекс связности бывает гомотопический и гомологический. Гомологическим индексом связности комплекса $K$ назьвается наименьшая из размерностей его негомологичных нулю целочисленных циклов; обозначение: $\operatorname{cin} K$ (cin = connectivity index). Гомотопический индекс связности естественно определить как размерность первой нетривиальной групшы гомотопий. Для односвязных комплексов гомотопический индекс совпадает с гомологическим в силу теоремы Гуревича. Это верно также для гомотопически простых комплексов, которые мы, следуя М. М. Постникову [32], будем называть абелевыл ми. Мы в дальнейшем будем заниматься в основном гомологическим индексом, назьвая его просто индексом связности. Хотелось бы называть индекс сөязности еще проще - сөязностью. Это, однако, может привести к путанице, так как в устоявшейся терминологии $n$-связным называют пространство, у которого нулевые все групшы гомотопий включая $n$-ю.

Поскольку мы рассматриваем приведенные гомологии, естественно работать в категории пунктированных комплексов, что мы и делаем в дальнейшем, не акцентируя на этом внимания. Произведением в категории пунктированных пространств является приведенное произведение, обозначаемое $M \wedge N=M \times N / M \vee N$ (smash-product), а суммой в категории пунктированных пространств является букет $M \vee N$. Поскольку $\operatorname{cin} S^{n}=n$ и $S^{n} \wedge S^{m}=S^{n+m}$, возникает естественное предположение, что индекс связности удовлетворяет логарифмическому закону $\operatorname{cin} M \wedge N=\operatorname{cin} M+\operatorname{cin} N$. Следующая "естественная" гипотеза заключается в том, что гомологический индекс связности не зависит от группы коэффициентов.

Эти проблемы, так же как их аналоги для компактов, имеют отрицательное решение. Причем уже первый шаг конструкции понтрягинских поверхностей опровергает как логарифмический закон для связностей, так и гипотезу о равносильности коэффициентов. А именно, если лист Мёбиуса по модулю $p$ обозначить $M_{p}$, то $\operatorname{cin} M_{p}=1$ для любого $p$ и $\operatorname{cin}\left(M_{p} \wedge M_{q}\right)=\infty$ при $p \neq q$. После этого по аналогии с компактами можно было бы ввести понятие связно-полноценного комплекса и поставить вопрос о критерии связной полноценности. Критерий получается такой же, как у Болтянского: $\operatorname{cin}_{\mathbb{Q}_{p}} M=\operatorname{cin} M$ для любого $p$. И доказьвается, так же как у Болтянского, на основе примера комплекса Мура $M\left(\mathbb{Q}_{p}, 1\right)$, вьполняющего в категории комплексов роль компакта Болтянского, для которого $\operatorname{cin} M\left(\mathbb{Q}_{p}, 1\right)=1$ и $\operatorname{cin} M\left(\mathbb{Q}_{p}, 1\right) \wedge$ $M\left(\mathbb{Q}_{p}, 1\right)=3$. Связно-полноценные типы находятся во взаимнооднозначном соответствии с натуральными числами и представляются сферами.

После этого можно было бы задаться вопросом о коэффициентном базисе. Им оказывается та же самая система Бокштейна. Потом получить аналоги теорем Бокштейна. Все это легко можно сделать, и все это сделано в первой главе.

По аналогии с теорией размерности определим сөязностный тuп комплекса. А именно, два комплекса $K, L$ называем имеюшими одинаковый связностный тип и пишем CIN $K=\operatorname{CIN} L$, если $\operatorname{cin} K \wedge M=\operatorname{cin} L \wedge M$ для любого комплекса $M$.

Множество связностных типов естественно упорядочено и имеет операцию, инду- 
цированную операцией приведенного произведения. Операция эта обозначается $\langle+\rangle$. Таким образом, $\operatorname{CIN}(M \wedge N)=\operatorname{CIN} M\langle+\rangle \operatorname{CIN} N$. Упорядоченное множество размерностных типов с этой операцией назьвается связностной алгеброй и обозначается $\mathfrak{C N}$. В отличие от размерностной алгебры, проблема реализации решается в связностной алгебре без труда. А именно, комплексы Эйленберга-Маклейна $K(G, n)$ при $G$ из системы Бокштейна образуют базис этой алгебры, и всякий комплекс имеет связностньй тип букета таких комплексов (см. [29]).

Двойственность Дранишникова. Двойственность между размерностью и связностью проявляется и в рамках двойственности Александера-Понтрягина, и в обобщающей ее двойственности Спеньера-Уайтхеда. Собственно, уже канторовское определение кривой как замкнутого нигде не плотного множества плоскости, так вдохновившее Урысона, представляет собой определение размерности замкнутого множества через связностные свойства его дополнения (множество, имеющее непустую внутренность, можно трактовать как локально зацепленное в размерности -1 ).

Рассмотрение отношения экстенсивности привело Дранишникова к открытию новой двойственности между компактами и комплексами, выходяшей за рамки двойственности Спеньера-Уайтхеда и вписьвающейся в категорную двойственность Экмана-Хилтона. Назовем әкстсвязностью комплекса $K$ множество топологических типов экстенсивных по отношению к нему компактов (обозначение: $\mathfrak{C i n} K$ ). Тогда оказывается, что для всякого компакта $X$ среди всех коэкстенсивных ему комплексов существует имеющий наименьшую экстсвязность (может быть несчетный). Назовем его экстсвязность двойственной экстмерности компакта $X$. И для всякого комплекса $K$ среди экстенсивных по отношению к нему компактов сушествует имеющий наименьшую экстмерность (возможно, неметризуемый). Назовем его экстмерность двойственной экстсвязности комплекса $K$. Тогда описанные отображения двойственности взаимно обратны и устанавливают сохраняющее порядок взаимнооднозначное соответствие между решетками экстмерности и экстсвязности [29]. Мы будем назьвать ее әкстенсивной двойственностью или двойственностью, Дранишникова.

В связи с этой двойственностью имеется следующая важная проблема, являющаяся аналогом проблемы нахождения счетного коэффициентного базиса, решенной Бокштейном.

ПРОБЛЕМА СЧЕТНОСТИ. Всякий ли комплекс имеет экстсвязность счетного?

Этой проблеме посвяшена статья Дранишникова [33], содержашаяся в этом же номере журнала. Если при определении экстенсивной двойственности ограничиться рассмотрением конечномерных компактов и гомотопически простых комплексов, то она превратится в гомологическую размерностно-связностную двойственность. А именно, отношение экстенсивности индуцирует отношение между размерностными и связностными типами, что следует из критерия экстенсивности Дранишникова (который верен не только для односвязных, но и для абелевых комплексов). И это отношение порождает отображение двойственности $\tau: \mathfrak{D M} \rightarrow \mathfrak{C N}$ так, что размерностному типу соответствует наименьший связностньй тип, содержащий комплекс, коэкстенсивньй компактам этого типа.

Отображение $\tau$ индуцирует биекцию размерностной и связностной алгебр, сохраняюшую порядок. Естественно было предполагать, что оно сохраняет и операции. Свойство сохранения операций посредством $\tau$ являлось бы экстмерньм вариантом 
логарифмического закона. Но и в этой форме логарифмический закон не состоялся. Это обстоятельство было открыто Дранишниковым [34], и именно оно стимулировало алгебраические исследования автора, которьй долго не мог в это поверить.

Двойственность Дранишникова склеивает размерностную алгебру со связностной. Получившийся объект мы называем размерностно-связностной алгеброй и обозначаем $\mathfrak{D C}$. Размерно-полноценным типам при этом соответствуют связно-полноценные. Эти “дважды полноценные” элементы размерностно-связностной алгебры отождествляются с натуральньми числами.

Операцию, индуцированную на $\mathfrak{D C}$ из $\mathfrak{D M}$, обозначаем [+], а операцию, индуцированную из $\mathfrak{C N}$, обозначаем $\langle+\rangle$. Эти операции нередко совпадают. Например, для любого $x \in \mathfrak{D C}$ имеем $x\langle+\rangle x=x[+] x$ и $x[+] n=x\langle+\rangle n$ для любого натурального $n$. И для любых $x$ и $y$ выполняется неравенство $x[+] y \leqslant x\langle+\rangle y$. Это неравенство оправдьвает выбор названия минорная для операции $[+]$ и мажсорная для операшии $\langle+\rangle$.

Автору не удалось обнаружить в алгебре такого типа структуры, которую имеет размерностно-связностная алгебра. И он дал ей имя биаддитивной решетки.

Тензорные типы. Начинается статья с реинтерпретации основных результатов Бокштейна [10], [11]. Этим результатам, чисто алгебраическим по содержанию, удалось найти и чисто алгебраическую форму. При этом оказалось, что вся теория Бокштейна касается лишь тензорных произведений абелевых групп. А ее связь с гомологической алгеброй и топологией осуществляется через формулы универсальных коэффициентов и Кюннета.

Вопрос, на которьй дает ответ Первая Теорема Бокштейна, - это нахождение условий коэффичиентной эквивалентности абелевых групп.

Следующие два условия оказываются равносильными для абелевых групп $G$ и $H$ :

1) для любого компакта $X$ и любого натурального $n$ групшы когомологий $H^{n}(X, G)$ и $H^{n}(X, H)$ или обе тривиальны, или обе нетривиальны;

2 ) для любого комплекса $L$ и любого натурального $n$ группы гомологий $H_{n}(L, G)$ и $H_{n}(L, H)$ или обе тривиальны, или обе нетривиальны.

Групшы, удовлетворяюшие этим условиям, называются коэффициентно равносильжылм. Ясно, что как когомологические размерности компактов, так и гомологические связности комплексов относительно коэффициентно-равносильных групп совпадают.

Ключевую роль в наших рассмотрениях играет понятие тензорного типа абелевой групшы. А именно, мы говорим, что абелевы группы $G$ и $H$ имеют одинаковый тензорный mun, если при тензорном умножении этих групп на любую третью получаются либо две тривиальные, либо две нетривиальные группы. Для групп без кручения и для периодических групп коэффициентная равносильность оказьвается равнозначна тензорной. Для смешанных групп (т.е. груп, имеюших элементы как конечного, так и бесконечного порядков) ответ немного сложнее.

На множестве тензорных типов есть естественное отношение порядка. А именно, мы говорим, что группа $G$ тензорно сильнее группы $H$, если сумма $G \oplus H$ тензорно равносильна $G$. Тензорный тип назьвается неразложимымм, если никакую группу этого типа нельзя представить в виде суммы груп других тензорных типов. Ясно, что всякий минимальньй тип неразложим.

Порядковая структура множества неразложимых тензорных типов такова. Три- 
виальный тип представлен одной единственной тривиальной группой 0 и является наименьшим тензорным типом. Все множество типов делится на три непересекаюшиеся категории: минимальных, максимальных и полевых. Тензорньй тип назьвается минимальньм, если не существует типа тензорно более слабого, чем он. Минимальность автоматически влечет неразложимость типа. Оказьвается, что минимальные типы находятся во взаимнооднозначном соответствии с квазициклическими группами, т.е. каждая квазициклическая група имеет минимальньй тип, различные квазищиклические групшы имеют различные типы и всякий минимальньй тип является типом квазициклической групшы. Неразложимьй тензорный тип называется максимальнblм, если не сушествует более сильного неразложимого типа, чем он. Максимальные типы однозначно соответствуют кольцам $\mathbb{Z}_{(p)}$.

И, наконец, тензорный тип аддитивной группы поля называется полевым. Аддитивные групшы полей имеют одинаковый тензорный тип в том и только том случае, когда у них совпадают характеристики. Таким образом, полевые типы находятся в естественном соответствии с множеством простых чисел, дополненным нулем. Тензорньй тип поля нулевой характеристики назьвается рачиональным. Прочие полевые типы соответствуют полям вычетов $\mathbb{Z}_{p}$. Таким образом, неразложимые тензорные типы оказьваются находящимися во взаимнооднозначном соответствии с группами системы Бокштейна, и мы поэтому будем в дальнейшем отождествлять тензорные типы групп с группами системы Бокштейна.

Рациональньй тип сильнее всех минимальных и слабее всех максимальных типов. На множестве остальных типов транзитивным является отношение сравнимости. Оно разбивает остальные типы на тройки $\mathbb{Q}_{p}, \mathbb{Z}_{p}, \mathbb{Z}_{(p)}$, выписанные в порядке возрастания тензорной силы. Каждой такой тройке естественно соответствует простое число, назьваемое ее факторностью. Факторность $\mathbb{Q}$ считается равной нулю.

Коэффициентньй тип групы $G$ определяется на основе ее $p$-факторов Бокштейна. $p$-фактор Бокштейна групш $G$ определяется как тензорньй тип $p$-локализации $G \otimes \mathbb{Z}_{(p)}$, от которого “отшеплен” рациональный фактор. То есть, в случае, когда из локализации выделяется $\mathbb{Q}$ в качестве прямого слагаемого, в качестве $p$-фактора нужно рассмотреть тензорньй тип ее кручения. $\sigma_{p} G$ либо тривиально, либо имеет тип одной из бокштейновских групп $\mathbb{Z}_{(p)}, \mathbb{Q}_{p}, \mathbb{Z}_{p}$.

Нетривиальность бокштейновского $p$-фактора $\sigma_{p} G$ обусловлена нарушением делимости на $p$ в группе $G$. Нарушения делимости бывают трех типов, первый - когда группа содержит элемент бесконечного порядка, не деляшийся на $p$, второй - когда она содержит элемент конечного порядка, не делящийся на $p$, и третий - когда все элементы групшы делятся на $p$, но деление неоднозначно. Этим трем случаям как раз и соответствуют три тензорных типа $\sigma_{p} G$.

Оказьвается, что справедливо следующее утверждение.

КРИТЕРИЙ КОЭФФИЦИЕНТНОЙ РАВНОСИЛЬНОСТИ. Абелевы грynnы $G$ u әффициентно равносильны в том и только том случае, когда они имеют совпадающие бокштейновские р-факторы при любом $p \geqslant 0$.

Из этого критерия вытекает, что всякая группа коэффициентно эквивалентна сумме своих $p$-факторов. А поскольку когомологии и гомологии аддитивны по отношению 
к коэффициентам, постольку знание размерностей компакта по отношению к неразложимьм тензорньм типам позволяет определить размерности по отношению к любым. Таким образом, этот критерий отвечает на те же вопросы, что и Первая Теорема Бокштейна.

Алгебры Кюннета. Вопрос, на который отвечает Вторая Теорема Бокштейна, о вычислении размерностей произведения - также, оказывается, может быть отделен не только от топологии, но и от гомологической алгебры.

Последовательность абелевых груш $\left\{G_{0}, G_{1}, G_{2}, \ldots\right\}$ назьвается полигруппой. ${ }^{3}$ Почленное сложение превращает множество полигрупп в абелев моноид. Всякое умножение на группах индуцирует умножение на полигруппах. А именно, $n$-й член произведения полигруп $\left\{G_{i}\right\}$ и $\left\{H_{j}\right\}$ задается посредством формулы свертки как сумма произведений $G_{i} H_{j}$ для $i+j=n$. Так, тензорное и периодическое произведения групп индуцируют тензорную и периодическую свертки на полигруппах. А те умножения, которые нас интересуют в этой статье, являются суммой тензорной и сдвинутой на единицу периодической сверток. Поскольку сдвигать на единицу можно как вверх, так и вниз, постольку возникают две операции, назьваемые верхним и нижним произведениями Кюннета. Верхнее произведение соответствует формуле Кюннета для когомологий, а нижнее - для гомологий.

На множестве полигрупп имеются две естественные градуировки. Высотой или размерностью полигрупшы назьвается номер ее наибольшего нетривиального члена, а глубиной или связностью - наименьшего. Эти градуировки, подобно размерности и связности, позволяют определить высотный и глубинный типы полигрупп. Причем высотньй тип определяется посредством операции верхнего произведения, а глубинньц̆ - нижнего.

Вся теория Бокштейна, по сушеству, заключается в описании верхней алгебры Кюннета - алгебры высотных типов полигрупп. Описание Бокштейна заключается в следуюшем: высотный тип полигрупшы $\mathscr{G}$ однозначно определяется функциями Бокштейн $а$, которые каждой группе $C$ из системы Бокштейна ставят в соответствие высоту произведения Кюннета полигрупшы $\mathscr{G}$ и полигруппы $\{C, 0,0, \ldots\}$ (=размерность с коэффициентами в $C$ ). И Вторая Теорема Бокштейна дает формулу для вычисления функции Бокштейна кюннетовского произведения полигруп.

Альтернативное описание алгебр Кюннета, предложенное в $§ 2$ настояшей работы, заключается в том, чтобы вместо размерностей относительно коэффициентов использовать тензорные типы групп. А именно, оказьвается, что высотный тип полигрупшы $\left\{G_{n}\right\}$ однозначно определяется значениями ее высших р-факторов Бокштейна, т.е. тензорным типом $\sigma_{p} G_{n}$ для наибольшего $n$, при котором он нетривиален. А высшие $p$-факторы произведения полигрупп вычисляются через таблицу умножения факторов размером $3 \times 3$.

В $\S 6$ настоящей статьи показано, что размерностная алгебра изоморфна верхней алгебре Кюннета, а связностная - нижней. В случае связностной алгебры изоморфизм задается обычньм функтором гомологий. В случае размерностной алгебры он осушествляется тотальными когомологиями $\mathscr{H}^{*}(X)$, определяемыми для компакта $X$ как сумма целочисленных когомологий пар $H^{*}(X, A)$ по всем замкнутым подмножествам $A \subset X$.

\footnotetext{
${ }^{3} \mathrm{~B}$ литературе принят термин градуированная группа.
} 
Триплетные кодировки. Для факторного описания полигрупп мы кодируем их посредством так назьваемых триплетов. Триплетнылми кодами или, кратко, триплетами мы назьваем элементы последовательности

$$
\mathbb{N}^{ \pm}=\left\{0,1,1^{+}, 2^{-}, 2,2^{+}, \ldots, n^{-}, n, n^{+}, \ldots, \infty\right\}
$$

Триплеты делятся на три типа: со знаком минус назьваются минорнылми, со знаком плюс мажсорными, а без знака регулярнылми или натуральнылми. Последних мы отождествляем с натуральными числами. Натуральное число, получающееся при отбрасьвании знака триплета, назьвается его основой.

Размерностньй тип компакта кодируется последовательностью триплетов - кодов высших $p$-факторов его тотальных когомологий. Возникаюшие при этом триплетные последовательности удовлетворяют следующим трем условиям:

1) если хотя бы один член последовательности равен нулю, то и все члены последовательности нули;

2) начальньй член последовательности (соответствующий 0-фактору) является натуральным числом;

3) если какой-то член последовательности является натуральным числом, то он совпадает с начальным членом.

Триплетные последовательности, удовлетворяюшие этим трем условиям, назьваются корректными кодировками. Отметим, что постоянная кодировка обязательно принимает натуральное значение. Постоянные кодировки отождествляются с натуральными числами. Множество корректных кодировок упорядочивается в соответствии с порядком, имеюшимся на триплетах (которые написаны вьше в порядке возрастания). А именно, одна кодировка считается больше либо равной другой, если при любом $p$ соответствуюший член первой кодировки больше либо равен соответствующего члена второй (почленное сравнение).

На триплетах вводятся две операции сложения - минорная $[+]$ и мажсорная $\langle+\rangle-$ следуюшим образом: $n[+] m^{ \pm}=(n+m)^{ \pm}=n\langle+\rangle m^{ \pm}, n^{ \pm}[+] m^{ \pm}=(n+m)^{ \pm}=$ $n^{ \pm}\langle+\rangle m^{ \pm}, n^{ \pm}[+] m^{\mp}=(n+m)^{-}$и $n^{ \pm}\langle+\rangle m^{\mp}=(n+m)^{+}$. То есть основы триплетов при сложении складьваются. Операции сложения на триплетах порождают аналогичные операции на кодировках. Минорная и мажорная суммы кодировок вычисляются почленно.

Совокупность ограниченных кодировок с описанньм вьше отношением порядка и операцией минорного сложения оказьвается изоморфной размерностной алгебре. Для компакта $X$ кодируюшую его высшие когомологические факторы последовательность обозначаем $\operatorname{Dim} X$. А через $\operatorname{Dim}_{p} X$ обозначаем член этой последовательности, кодируюший $p$-фактор. Основа триплета $\operatorname{Dim}_{p} X$ совпадает с размерностью по модулю $p$. А тип триплета $\operatorname{Dim}_{p} X$ определяется тензорным типом соответствующего $p$-фактора. Таким образом, аддитивность основ триплетов выражает логарифмический закон Понтрягина. Неравенство DIM $X \geqslant \operatorname{DIM} Y$ оказьвается равносильным неравенству $\operatorname{Dim} X \geqslant \operatorname{Dim} Y$, и имеет место формула произведения

$$
\operatorname{Dim}(X \times Y)=\operatorname{Dim} X[+] \operatorname{Dim} Y .
$$


А совокупность кодировок с тем же самым отношением порядка, но с мажорной суммой оказывается изоморфной связностной алгебре. Изоморфизм осуществляется посредством кодирующего отображения $\mathrm{Cin}$, которое получается посредством кодирования низиих гомологических $p$-факторов Бокштейна.

Замечательно простой вид приобретает в результате кодирования критерий экстенсивности Дранишникова:

$$
X \tau K \Longleftrightarrow \operatorname{Dim} X \leqslant \operatorname{Cin} K .
$$

Неравенство Урысона-Дыдака приобретает вид

$$
\operatorname{Dim}(X \cup Y) \leqslant \operatorname{Dim} X\langle+\rangle \operatorname{Dim} Y+1 \text {. }
$$

Под единицей в данной формуле подразумевается единичная кодировка, а знак + поставлен без скобочек, потому что минорная и мажорная суммы совпадают в случае, когда одно из слагаемых натурально. Формула джойна комплексов вьплядит так:

$$
\operatorname{Cin}(K * L)=\operatorname{Cin} K\langle+\rangle \operatorname{Cin} L+1
$$

И, наконец, главная формула этой статьи - формула пересечения - вьглядит так:

$$
\operatorname{Dim}(f(X) \cap g(Y)) \leqslant(\operatorname{Dim} X[+] \operatorname{Dim} Y)\langle->n .
$$

Эта формула справедлива для почти всех ${ }^{4}$ отображений компактов $X, Y$ в $\mathbb{R}^{n}$ в случае, если $\operatorname{dim} X, \operatorname{dim} Y \leqslant n-2$. В последней формуле фигурирует мажорная разность. Вот ее определение: $C\langle-\rangle=\inf \{B \mid B\langle+\rangle C \geqslant D\}$. Парная ей минорная разность определяется так: $\mathrm{C}[-] D=\sup \{B \mid B[+] C \leqslant D\}$. Используя понятие мажорной разности, можно сформулировать такой вариант теоремы Урысона-Дранишникова: для любой кодировки $D$ в компакте $X$ найдется такое сигма-компактное $X^{D}$, что $\operatorname{Dim} X^{D} \leqslant D u \operatorname{Dim} Y \leqslant \operatorname{Dim} X\langle-\rangle(D+1)$ для любого компакта $Z \subset X \backslash X^{D}$.

Размерности пересечений. Кодовая алгебра $\mathfrak{C D}$ определяется как множество корректных кодировок с введенным выше отношением порядка и парой операций сложения [+], $\langle+\rangle$. Эти две операции сложения, как будет показано в статье, при любых $B, C, D$ удовлетворяют следующему неравенству парной ассоциативности:

$$
D[+](C\langle+\rangle B) \leqslant(D[+] C)\langle+\rangle B .
$$

Оказывается, что знания этого неравенства достаточно для того, чтобы проводить вычисления в кодовой алгебре. Таковые вычисления в значительном количестве приходится проводить в третьей главе, посвященной теории пересечений компактов.

Размерность пересечения компактов $X_{1}, X_{2}, \ldots, X_{k}$, находяшихся в общем положении в $n$-мерном евклидовом пространстве, как показано в этой статье, выражается следующей формулой

$$
\operatorname{dim}\left(X_{1} \cap X_{2} \cap \cdots \cap X_{k}\right)=\operatorname{dim}\left(X_{1} \times X_{2} \times \cdots \times X_{k}\right)-(k-1) n .
$$

\footnotetext{
${ }^{4} \mathrm{~B}$ этой статье почти все значит дополнение имеет первую категорию.
} 
Для $k=2$ этот результат доказан в статье [19]. Но из результата для $k=2$ нельзя вывести результат для $k=3$, потому что он дает неполную информацию о размерностном типе пересечения. Полная информация должна в себя включать когомологические размерности пересечения по всем бокштейновским группам. В “бокштейновских координатах" размерностный тип пересечения записьвается ненамного сложнее размерностного типа произведения. Но и бокштейновское представление размерностного типа произведения не отличается простотой. И сложность выражения размерностей по бокштейновским группам для многократного пересечения растет с ростом кратности.

Триплетный формализм является, альтернативньм бокштейновскому, координатным представлением размерностно-связностной алгебры. Но оказывается, что вычисления в этой алгебре можно проводить, вообе не прибегая к координатам.

Так, формула для пересечения трех компактов получается из формулы парного пересечения следующей выкладкой:

$$
\begin{aligned}
\operatorname{Dim}\left(X_{1} \cap X_{2} \cap X_{3}\right) & =\left(\left(\operatorname{Dim}\left(X_{1} \cap X_{2}\right)\langle->n)[+] \operatorname{dim} X_{3}\right)\langle-\rangle n\right. \\
& =\left(\left(\left(\operatorname{Dim} X_{1}[+] \operatorname{Dim} X_{2}\right)\langle->n)[+] \operatorname{Dim} X_{3}\right)\langle-\rangle n\right. \\
& =\left(\operatorname{Dim} X_{1}[+] \operatorname{Dim} X_{2}[+] \operatorname{Dim} X_{3}\right)\langle->2 n .
\end{aligned}
$$

Доказательство последнего равенства занимает три строчки, оно проводится “бескоординатно" и использует обшие свойства операций сложения $[+],\langle+\rangle$ и вычитания $[-],\langle-\rangle$, а также то обстоятельство, что $n[-] D=n\langle-\rangle D$ для кодировки размерности $\leqslant n-1$. Последнее обстоятельство и приводит к ограничению на коразмерность компактов. Причина, по которой эти выкладки не проходят для компактов коразмерности 1 , заключается в отсутствии $1^{-}-$минорной единицы - среди триплетов. Таким образом, формула для размерности пересечения нескольких компактов выводится из формулы пересечения для двух.

Все вычисления, проведенные в этой статье при получении основных результатов по теории пересечений компактов, имеют такой бескоординатный характер. Понятие размерности по группе коэффициентов почти не применяется при таком подходе к изложению когомологической теории размерности.

Структура статьи. Статья начинается с алгебры. Для понимания ее алгебраической части от читателя требуется лишь элементарное знакомство с понятием тензорного и периодического произведения абелевых групп. Знание теории Бокштейна не предполагается, она полностью переизложена в алгебраической части. Третий параграф можно читать независимо от двух предыдущих.

Начинать чтение статьи можно также со второй главы. Четвертый параграф̆ не зависит от алгебры. Для его понимания от читателя требуются лишш элементарные знания из теории непрерывного продолжения отображений. В этом параграфе изложены все основные результаты теории экстмерности. И он может служить введением в эту теорию.

Завершаюшая глава посвящена теории пересечений. Эта геометрическая глава опирается на все предыдушие результаты. Но $\S 7$ совершенно элементарен и может быть прочитан независимо от всего остального. Для понимания $\S 8$ и $\S 9$ необходимо 
владение размерностными кодировками. Хотя понятие ручного компакта и появляется начиная с $\S 8$, применения теории М. А. Штанько удается избежать вплоть до заключительного раздела $\S 9$.

Параграф, посвященный триплетному кодированию (§3), хотя и опирается на результаты первой главы, может быть прочитан независимо. В этом параграфе необходимо научиться технике вычислений в кодовой решетке. Эта техника необходима для понимания последней главы.

Наименее элементарен пятьй параграф, посвященньй доказательству критерия экстенсивности Дранишникова. От читателя здесь требуется как минимум владение теорией препятствий.

\section{Глава 1. Алгебра}

\section{§ 1. Тензорная классификация абелевых групп}

Терминология. Через $\mathfrak{P}$ и $\mathfrak{P}_{0}$ обозначаются множество простых чисел и простых чисел с нулем. Для простого $p$ и группы $G$ ее $p$-компонентой назьвается подгруппа $\operatorname{Tor}_{p} G \subset G$, состоящая из элементов, порядок которых является степенью числа $p$. Подгрупа кручения $\operatorname{Tor} G$, составленная всеми элементами конечного порядка, является прямой суммой $p$-компонент: Tor $G=\bigoplus_{p \in \mathfrak{P}} \operatorname{Tor}_{p} G$.

И мы назьваем факторгруппу $G$ / Tor $G$ ее 0-компонентой и обозначаем $G^{0}$. Число $p$ (нуль или простое) будем называть периодом группы $G$, если $p$-компонента $G$ нетривиальна. Множество всех периодов группы обозначается $\mathscr{T}(G)$ и назьвается ее периодическим спектром. Группу, периодический спектр которой состоит ровно из одного числа, будем назьвать монопериодической. Монопериодическая группа периода $p$ совпадает со своей $p$-компонентой и называется $p$-группой при $p>0$.

Простое число $p$ назьваем делителем групшы $G$, если $G=p G$, т.е. каждый элемент группы $G$ делится на $p$. Группа, имеющая $p$ делителем, назьвается $p$-делимой. Множество всех делителей групш $G$ обозначается $\mathscr{D}(G)$.

Групп, состоящую из одного элемента, назьваем тривиальной или нулевой и обозначаем 0. Нулевая группа 0 характеризуется отсутствием периодов, так что $\mathscr{D}(0)=\mathfrak{P}$ и $\mathscr{T}(0)=\varnothing$.

Группа называется делимой, если $\mathscr{D}(G)=\mathfrak{P}$. Напомним, что $p$-группа делима на любое простое $q \neq p$, поэтому ее делимость равносильна $p$-делимости.

Основные группы. Аддитивные группы целых и рациональных чисел обозначаются $\mathbb{Z}$ и $\mathbb{Q}$. Циклическая группа порядка $p$ обозначается $\mathbb{Z}_{p}$. Несократимая дробь $m / n$ назьвается $p$-целым числом, если ее знаменатель взаимно прост с $p$. Через $\mathbb{Z}_{(p)}$ обозначается подгрупа рациональных чисел, состоящая из $p$-целых чисел. Через $\mathbb{Q}_{p}=\mathbb{Z}_{(p)} / \mathbb{Z}$ обозначается квазициклическая $p$-група.

Совокупность групп $\left\{\mathbb{Q}, \mathbb{Q}_{p}, \mathbb{Z}_{p}, \mathbb{Z}_{(p)}\right\}_{p \in \mathfrak{P}}$ обозначается $\sigma$ и назьвается системой Бокштейна. Групшы $\mathbb{Q}_{p}, \mathbb{Z}_{p}, \mathbb{Z}_{(p)}$ будем назьвать бокштейновскими факторнос$m u$, а рациональные числа - факторности 0.

Для бокштейновских групп нетрудно проверить следуюшие свойства:

$$
\begin{aligned}
& \mathscr{D}\left(\mathbb{Z}_{(p)}\right)=\mathscr{D}\left(\mathbb{Z}_{p}\right)=\mathfrak{P} \backslash p, \quad \mathscr{D}(\mathbb{Q})=\mathscr{D}\left(\mathbb{Q}_{p}\right)=\mathfrak{P}, \\
& \mathscr{T}\left(\mathbb{Z}_{(p)}\right)=\mathscr{T}(\mathbb{Q})=\varnothing, \quad \mathscr{T}\left(\mathbb{Z}_{p}\right)=\mathscr{T}\left(\mathbb{Q}_{p}\right)=\{p\} .
\end{aligned}
$$


Тензорная сила. Индексом тривиальности групшы $G$ будем назьвать число, обозначаемое через $\operatorname{triv} G$ и равное нулю, если группа $G=0$, и единице в противном случае.

Мы говорим, что тензорная сила группы $G$ не уступает тензорной силе групшы $H$, и записьваем это отношение $G \succ H$, если $\operatorname{triv} G \otimes F \geqslant \operatorname{triv} H \otimes F$ для любой $F$. Групшы равной тензорной силы назьваются тензорно әквивалентнылми, и отношение тензорной эквивалентности обозначается так: .

Из дистрибутивности операции тензорного произведения по отношению к прямой сумме груп непосредственно вытекает соотношение

$$
\operatorname{triv}\left(\left(G \oplus G^{\prime}\right) \otimes H\right)=\max \left\{\operatorname{triv}(G \otimes H), \operatorname{triv}\left(G^{\prime} \otimes H\right)\right\} .
$$

Прямая сумма индуцирует идемпотентную операцию $(G \oplus G \sim G)$ на множестве тензорных типов групп, согласованную с тензорньм порядком, так что неравенство $G \succ G^{\prime}$ равносильно равенству $G \oplus G^{\prime} \sim G$. Тензорное произведение также индуцирует операщию на множестве тензорных типов, как вытекает из следуюшего утверждения.

О ПРОИЗВЕДЕНИИ ТИПов. Если $G \sim G^{\prime} u H \sim H^{\prime}$, mo $G \otimes H \sim G^{\prime} \otimes H^{\prime}$.

ДокАЗАТЕльство заключается в цепочке равенств: $\operatorname{triv}(G \otimes H) \otimes F=\operatorname{triv} G \otimes$ $(H \otimes F)=\operatorname{triv} G^{\prime} \otimes(H \otimes F)=\operatorname{triv}\left(G^{\prime} \otimes F\right) \otimes H=\operatorname{triv}\left(G^{\prime} \otimes F\right) \otimes H^{\prime}=\operatorname{triv}\left(G^{\prime} \otimes H^{\prime}\right) \otimes F$.

Тензорно-периодическая последовательность. Всякая тройка групп $G^{\prime} \subset$ $G, H$ порождает следуюшую точную последовательность [35]

$$
0 \rightarrow G^{\prime} * H \rightarrow G * H \rightarrow G / G^{\prime} * H \rightarrow G^{\prime} \otimes H \rightarrow G \otimes H \rightarrow G / G^{\prime} \otimes H \rightarrow 0,
$$

гомоморфизмы которой являются тензорными и периодическими произведениями тождественного $\operatorname{id}_{H}: H \rightarrow H$ на соответствуюшие гомоморфизмы включения или факторизации пары $G^{\prime}, G$.

Тензорно-периодическая последовательность естественно делится на две половины, левую - периодическую и правую - тензорную, которые, вообще говоря, не являются точными. Периодическая последовательность точна слева, а тензорная - справа. Из этих обстоятельств непосредственно вытекают следуюшие соотношения между тензорной и периодической силами групшы, подгрупшы и фактор-групшы, которые мы будем именовать свойствами монотонности тензорного произведения по факторгруппам и периодического - по подгруппам.

СвойствА монотонности. Для любых групn $G^{\prime} \subset G, H$ справедливы неравенства: $\operatorname{triv}(G \otimes H) \geqslant \operatorname{triv}\left(G / G^{\prime} \otimes H\right) u \operatorname{triv}\left(G^{\prime} * H\right) \leqslant \operatorname{triv}(G * H)$.

Обращение в нуль последнего члена периодической части тензорно-периодической последовательности является, очевидно, необходимым и достаточным условием точности ее тензорной части, что и фиксирует следующее предложение.

УСЛОВИЕ ТЕНЗОРНОЙ ТОчностИ. Если $H * G / G^{\prime}=0$, то точна последовательность $0 \rightarrow G^{\prime} \otimes H \rightarrow G \otimes H \rightarrow G / G^{\prime} \otimes H \rightarrow 0$.

Это условие вьполнено, когда $H$ не имеет кручения. Таким образом, мы получаем такое следствие. 
Тензорная 0-монотонность. $\operatorname{triv} G^{\prime} \otimes H \leqslant \operatorname{triv} G \otimes H$, ecлu Tor $H=0$.

ТЕНЗОРНАЯ 0-НЕВЫРОЖДЕННОСТЬ. Тензорное произведение нетривиальных зрупп без кручения нетривиально.

ДокАЗАТЕЛЬСтво. Пусть $G$ и $H$ - две групшы без кручения. Тогда $G$ содержит подгруппу $G^{\prime}$, изоморфную $\mathbb{Z}$. И поскольку $G^{\prime} \otimes H=H \neq 0$, постольку в силу 0 -монотонности получаем $G \otimes H \neq 0$.

ОтШЕПлЯЕмость НУля. $G \sim G^{0} \oplus$ Tor $G$ для любой гpynnы $G$.

ДоКАЗАТЕльстВо. Поскольку $G^{0}$ не имеет кручения, постольку для любой группы $H$ точна последовательность

$$
0 \rightarrow \operatorname{Tor} G \otimes H \rightarrow G \otimes H \rightarrow G^{0} \otimes H \rightarrow 0
$$

поэтому $\operatorname{triv}(G \otimes H)=\max \left\{\operatorname{triv}(\operatorname{Tor} G \otimes H), \operatorname{triv}\left(G^{0} \otimes H\right)\right\}$ определяется той же формулой, что и для $G^{0} \oplus \operatorname{Tor} G$.

\section{Свойства делителей групп}

Помимо тензорно-периодической последовательности есть два основных простых соображения, используемых при вычислениях тензорных произведений. Мы будем именовать их приниипом уничтожения и приниипом сохранения.

ПРИНцИП УНИчТожЕНИя. Если $g$ делится на порядок $h$, mо $g \otimes h=0$.

ДОКАЗАТЕЛЬСТВО. Если $n h=0$ и $g=n g^{\prime}$, то $g \otimes h=g^{\prime} \otimes n h=0$.

ПРИНЦИП СОХРАНЕНИЯ. Если группа $G$ является модулем над кольиом с единицей $R$, то соответствие $g \rightarrow g \otimes 1$ индуцирует изоморфизм группь $G$ на тензорное произведение аддитивной группы кольиа $G \otimes R$.

ДоКАЗАТЕЛЬСТво. Обратный гомоморфизм из $G \otimes R \rightarrow G$ задается модульньм умножением $(r \otimes g) \rightarrow r g$.

Поскольку всякая $p$-группа делима на любое простое $q$, отличное от $p$, из принципа уничтожения получаем такое следствие:

Тензорное произведение $p$-группь на q-группу тривиально, если $p \neq q$.

Во всякой $p$-группе $G$ уравнение $n x=g$ имеет единственное решение, если $n$ не делится на $p$. Таким образом, возможно определить умножение $p$-целых рациональных чисел на элементы групшы, т.е. превратить ее в модуль над кольцом $\mathbb{Z}_{(p)}$. Тензорное произведение $G \otimes \mathbb{Z}_{(p)}$ назьвается $p$-локализацией групшы $G$.

ЛЕМма о ЛОКАЛИЗАцИИ. Если $G^{0}=0, m o G \otimes \mathbb{Z}_{(p)}=\operatorname{Tor}_{p} G$.

ДокАЗАТЕльствО. Поскольку $\mathbb{Z}_{(p)}$ делится на любое простое $q$, отличное от $p$, постольку $\mathbb{Z}_{(p)} \otimes \operatorname{Tor}_{q} G=0$ в силу принципа уничтожения. И $\mathbb{Z}_{(p)} \otimes \operatorname{Tor}_{p} G=\operatorname{Tor}_{p} G$ в силу принципа сохранения. Откуда и следует нужное утверждение.

КРИТЕРИЙ ДЕЛИМОСТИ. $p \in \mathscr{D}(G)$, если и только если $G \otimes \mathbb{Z}_{p}=0$. 
ДокАЗАТЕЛЬСТво. Это так ввиду изоморфизма $\mathbb{Z}_{p} \otimes G=G / p G$ (см. [35]).

Из этого критерия вытекает, что свойство $p$-делимости является инвариантом тензорного типа. То есть групш, имеюшие одинаковьй тензорньй тип, имеют одинаковые множества делителей.

ТЕОРемА О ДЕЛИТЕЛЯХ. Для любъх әрупп $G$ u

$$
\mathscr{D}(G \otimes H)=\mathscr{D}(G) \cup \mathscr{D}(H), \quad \mathscr{D}(G \oplus H)=\mathscr{D}(G) \cap \mathscr{D}(H) .
$$

ДокАЗАТЕЛЬСТВО. Мы ограничимся доказательством формулы для делителей произведения, оставив читателю формулу для делителей суммы. Если $G$ делится на $p$, то $G \otimes \mathbb{Z}_{p}=0$, и, значит, $G \otimes H \otimes \mathbb{Z}_{p}=0$, поэтому $G \otimes H$ делится на $p$ в силу критерия делимости. Пусть $G \otimes H$ делится на $p$, тогда $0=G \otimes H \otimes \mathbb{Z}_{p}=G \otimes \mathbb{Z}_{p} \otimes \mathbb{Z}_{p} \otimes H$. В группе $G \otimes \mathbb{Z}_{p}$ все элементы имеют порядок $p$, и она является $\mathbb{Z}_{p}$-модулем, т.е. векторньм пространством над полем $\mathbb{Z}_{p}$ и, следовательно, изоморфна прямой сумме груп $\mathbb{Z}_{p}$. То же самое относится и к группе $H \otimes \mathbb{Z}_{p}$. Поэтому предположение, что обе они отличны от нуля, приводит к противоречию с предположением, что они имеют нулевое тензорное произведение. Значит, одна из них ноль и, следовательно, делится на $p$.

КРИТЕРИЙ $(p \otimes p)$-ТРИВИАЛЬНОСТИ. Тензорное произведение двух р-групп тривиально тогда и только тогда, когда одна из них делима на р.

ДокАЗАТЕльство. Если одна из них делится на $p$, то произведение групп тривиально в силу принципа уничтожения. Если же обе не делятся на $p$, то и произведение не делится на $p$ в силу теоремы о делителях.

КРИТЕРИЙ $(0 \otimes p)$-ТРИВИАЛЬНОСТИ. Тензорное произведение р-группы $G$ на группу $Н$ без кручения равно нулю в том и только том случае, когда $p \in \mathscr{D}(H)$.

ДокАЗАТЕЛЬСтво. Если $H$ делима на $p$, то $G \otimes H=0$ в силу принщипа уничтожения. Если $H$ не делима на $p$, то $H \otimes \mathbb{Z}_{p} \neq 0$. А так как всякая $p$-группа содержит $\mathbb{Z}_{p}$ в качестве подгруппы, то свойство 0-монотонности тензорного произведения влечет $H \otimes G \neq 0$.

КРИТЕРИЙ ДЕЛИМОСТИ 0-КОМПОНЕНТЫ. Произведение $G \otimes \mathbb{Q}_{p}$ тривиально, если и только если $G^{0}$ делится на р.

ДокАЗАТЕльство. Если $G^{0}$ не делится на $p$, то $G^{0} \otimes \mathbb{Q}_{p} \neq 0$ в силу критерия $(0 \otimes p)$-тривиальности. А значит, и $G \otimes \mathbb{Q}_{p} \neq 0$ в силу монотонности тензорного произведения по факторгруппам. Если же $G^{0}$ делится на $p$, то $G^{0} \otimes \mathbb{Q}_{p}=0$ и Tor $G \otimes$ $\mathbb{Q}_{p}=0$ в силу критериев $(p \otimes q)$ - и $(p \otimes p)$-тривиальности. Отсюда $G \otimes \mathbb{Q}_{p}=0$ в силу леммы об отшеплении нуля.

\section{Классификация монопериодических групп}

Следуюшее предложение решает проблему тензорной классификации для монопериодических групп.

КРИТЕРИЙ ДЕЛИТЕЛЕЙ. Монопериодические группь $G$ и $H$ тензорно әквивалентны $G \sim H$, если и только если $\mathscr{D}(G)=\mathscr{D}(H)$. 
ДокАЗАТЕЛЬСТвО. То, что тензорно эквивалентные группы имеют совпадаюшие множества делителей, уже отмечалось вьше (см. критерий делимости). Предположим теперь, что $G$ и $H$ являются монопериодическими групами периода $p$, возможно равного нулю, у которых совпадают множества делителей. Докажем их тензорную эквивалентность.

Если $p=0, G \neq 0$ и группа $F$ такова, что $F \otimes G=0$, тогда $F^{0}=0$ в силу 0 -невырожденности тензорного произведения и леммы об отщеплении 0-компоненты. Но тогда $F$ является прямой суммой своих компонент кручения. Следовательно, $G \otimes$ $\operatorname{Tor}_{q} F=0$ для всякого простого $q$. Последнее в силу критерия $(0 \otimes p)$-тривиальности означает, что $\mathscr{T}(F) \subset \mathscr{D}(G)$. Но тогда и $\mathscr{T}(F) \subset \mathscr{D}(H)$, и согласно тому же критерию $H \otimes F=0$.

Если $p>0, G \neq 0$ и $p \notin \mathscr{D}(G)$, а группа $F$ такова, что $F \otimes G=0$, то $F$ делится на $p$, и потому тензорное произведение групшы $F$ на любую $p$-группу тривиально и, в частности, $F \otimes H=0$.

Если $p>0, G \neq 0$ и $p \in \mathscr{D}(G)$, а группа $F$ такова, что $F \otimes G=0$, то как $G$, так и $H$ являются делимьми группами, и потому $H \otimes \operatorname{Tor} F=0$. Условие же $F^{0} \otimes G=0$ в силу критерия $0 \otimes p$-тривиальности равносильно делимости $p \in \mathscr{D}\left(F^{0}\right)$. И на основании этого же критерия $F^{0} \otimes H=0$, откуда и $F \otimes H=0$.

Поскольку множество делителей группы $\mathbb{Z}_{(p)}$ представляет собой все простые числа за исключением $p$, постольку из формул сложения для делителей и только что доказанного критерия делителей немедленно вытекает такое следствие:

ТЕНЗОРНАЯ КЛАССИФИКАЦИЯ ГРУПП БЕЗ КРУЧЕНИЯ. Если Тor $G=0$ u $\mathscr{D}(G) \neq$ $\mathfrak{P}$, mo $G \sim \bigoplus_{p \notin \mathscr{D}(G)} \mathbb{Z}_{(p)}$. Ecлu Tor $G=0$ u $\mathscr{D}(G)=\mathfrak{P}$, mo $G \sim \mathbb{Q}$.

А поскольку для $p$-групшы ( $p>0)$ все простые, за исключением, быть может, $p$, являются ее делителями, постольку из критерия делителей вытекает такое следствие:

ПРИМАРНАЯ ТЕНЗОРНАЯ КЛАССИФИКАЦИЯ. Всякая р-группа $G$ тензорно әквивалентна либо ииклической $\mathbb{Z}_{p}$, либо квазичиклической $\mathbb{Q}_{p}$ әруппе.

Приведенных вьше соображений хватает для вычисления тензорных произведений всех бокштейновских групп. Таблища таких произведений для групп факторности $p$ приведена ниже.

Тензорная таблица умножения

\begin{tabular}{|c|c|c|c|}
\hline & $\mathbb{Z}_{(p)}$ & $\mathbb{Z}_{p}$ & $\mathbb{Q}_{p}$ \\
\hline $\mathbb{Z}_{(p)}$ & $\mathbb{Z}_{(p)}$ & $\mathbb{Z}_{p}$ & $\mathbb{Q}_{p}$ \\
\hline $\mathbb{Z}_{p}$ & $\mathbb{Z}_{p}$ & $\mathbb{Z}_{p}$ & 0 \\
\hline $\mathbb{Q}_{p}$ & $\mathbb{Q}_{p}$ & 0 & 0 \\
\hline
\end{tabular}

Произведение бокштейновских групп различных факторностей нулевое, если только обе они не являются группами без кручения, и равно $\mathbb{Q}$, если они таковыми являются. Изоморфизм $\mathbb{Z}_{(p)} \otimes \mathbb{Z}_{(q)}=\mathbb{Q}$ устанавливается отображением $x \otimes y \rightarrow x y$. 
Периодическая классификация групп. Известно [35], что периодическое произведение любой группы $G * \mathbb{Z}_{p}$ на циклическую изоморфно подгрупе $G$, состояшей из элементов порядка $p$. Этого соображения хватает для заполнения следуюшей таблищы:

Периодическая таблища умножения

\begin{tabular}{|c|c|c|c|}
\hline & $\mathbb{Z}_{(p)}$ & $\mathbb{Z}_{p}$ & $\mathbb{Q}_{p}$ \\
\hline $\mathbb{Z}_{(p)}$ & 0 & 0 & 0 \\
\hline $\mathbb{Z}_{p}$ & 0 & $\mathbb{Z}_{p}$ & $\mathbb{Z}_{p}$ \\
\hline $\mathbb{Q}_{p}$ & 0 & $\mathbb{Z}_{p}$ & $\mathbb{Q}_{p}$ \\
\hline
\end{tabular}

НЕВЫРОЖДЕННОСТЬ ПЕРИОДИЧЕСКОГО ПРОИЗВЕДЕНИЯ. Периодическое произведение ненулевьх р-групп нетривиально.

ДокАзАТЕльство. Так как $\mathbb{Z}_{p} * \mathbb{Z}_{p} \cong \mathbb{Z}_{p}$, а всякая $p$-группа содержит $\mathbb{Z}_{p}$ как подгруппу, то $\operatorname{triv} G * H \geqslant \operatorname{triv} \mathbb{Z}_{p} * H \geqslant \operatorname{triv} \mathbb{Z}_{p} * \mathbb{Z}_{p}=1$ в силу отмеченной выше монотонности периодического произведения по подгруппам.

Две группы $G$ и $H$ называем периодически эквивалентными, если $\operatorname{triv}(G * F)=$ $\operatorname{triv}(H * F)$ для любой групшы $H$.

КРИТЕРИЙ ПЕРИОДИЧЕСКОЙ ЭКВИВАЛЕНТНОСТИ. Групnы $G$ u $H$ nериодически әквивалентны, если и только если $\mathscr{T}(G)=\mathscr{T}(H)$.

ДокАЗАТЕЛЬство. Как известно, периодическое произведение $p$-групшы на $q$-группу при различных простых $p \neq q$ тривиально [35]. Поэтому нужное нам утверждение вытекает из доказанной выше леммы о нетривиальности периодического произведения.

\section{Факторная классификация}

Число $(G, H)=\max \{3 \operatorname{triv}(G \otimes H), 2 \operatorname{triv}(G * H)\}$ назовем индексом кофакторности групп $G$ и $H$. Групшы, имеюшие нулевой индекс кофакторности, назьваются взаимно простыми. Как нетрудно видеть, взаимно простые групшы не могут иметь обших нетривиальных $p$-компонент, что оправдьвает этот термин.

Будем назьвать групшы $G$ и $H$ изофакторнымми, если $(G, F)=(H, F)$ для любой групшы $F$, и писать $G \approx H$. Если же для любой групшы $F$ выполняется неравенство $(G, F) \leqslant(H, F)$, то будем говорить, что группа $H$ факторно доминирует групу $G$, и писать $G \ll H$. Факторное доминирование, очевидно, влечет за собой тензорное. А тензорное и периодическое доминирование вместе влекут за собой факторное.

Неравенство $H \ll G$ расшифровывается следуюшим образом: если $H \otimes F \neq 0$, то и $G \otimes F \neq 0$, если же $H \otimes F=0$ и $H * F \neq 0$, то $(G \otimes F) \oplus(G * F) \neq 0$.

Дистрибутивность операций тензорного и периодического произведений по отношению к прямой сумме влечет следующее свойство дистрибутивности индекса кофакторности по отношению к прямой сумме. 
ДиСТРИБУТИВНость ИНДЕКСА КОФАКТорности. $\left(G, \bigoplus H_{n}\right)=\max \left(G, H_{n}\right)$.

Из этой дистрибутивности вытекает, что операция прямой суммы индуцирует операцию сложения на факторных типах групп. Так как 0-компонента никак не влияет на периодическое произведение, то равенство $G \sim G^{0} \oplus$ Tor $G$ влечет $G \approx G^{0} \oplus$ Tor $G$. Таким образом, имеет место следующее

КОМПОНЕНТНОЕ РАСШЕПЛЕНИЕ ФАКТОРНОГО ТИПА. Всякая группа факторно әквивалентна прямой сумме своих р-компонент $(p \geqslant 0)$.

Элементы $\mathscr{T}(G)$, входяшие в $\mathscr{D}\left(G^{0}\right)$, назьваются существенными периодами. Множество всех сушественных периодов обозначается через $\mathscr{T}^{0}(G)$. Тройку $\left\{\mathscr{D}(G), \mathscr{D}\left(G^{0}\right), \mathscr{T}^{0}(G)\right\}$ мы назьваем факторным спектром группы $G$. Для факторного спектра имеем равенство

$$
\mathscr{D}\left(G^{0}\right)=\mathscr{D}(G) \cup \mathscr{T}^{0}(G)
$$

Перейдем к факторной классификации абелевых групп. Для монопериодических груп факторная классификация совпадает с тензорной. Разница между этими понятиями проявляется лишш при сравнении смешанных групп. Для начала научимся сравнивать периодические группы с группами без кручения.

КРИТЕРИЙ СМЕШАННОГО СРАВНЕНИЯ. Если $G$ не имеет кручения, а $Н$ является р-группой и $p \notin \mathscr{D}(G)$, то $H \ll G$, если жее $p \in \mathscr{D}(G)$, то $G$ тензорно не сильнее чем $H$.

ДоКАЗАТЕльство. Если $p$ является делителем групшы $G$, то $G \otimes \mathbb{Z}_{p}=0$ и $H \otimes$ $\mathbb{Z}_{p} \neq 0$, поэтому неравенство $G \succ H$ не вьполнено. Если же $p$ не является делителем для $G$, то $G \succ G \otimes \mathbb{Z}_{(p)} \sim \mathbb{Z}_{(p)}$ в силу критерия делителей. Но $\mathbb{Z}_{(p)} \succ \mathbb{Z}_{(p)} \otimes H \sim H$, откуда $G \succ H$. Если же $G \otimes F=0$, то $\mathbb{Z}_{(p)} \otimes F=0$, поэтому $F$ является периодической группой без $p$-компоненты. Но тогда $F * H=0$ для $p$-группы $H$. Тем самьм доказано факторное доминирование $H \ll G$.

$p$-компоненту группы $G$ назовем несущественной, если $\operatorname{Tor}_{p} G \ll G^{0}$. Из критерия смешанного сравнения получаем

КРИТЕРИЙ СУЩЕСТВЕННОСТИ. р-компонента существенна тогда и только тогда, когда $p \in \mathscr{D}\left(G^{0}\right)$.

КОМПОНЕНТНЫЙ КРИТЕРИЙ ИЗОФАКТОРНОСТИ. Необходимым и достаточнымм условием изофакторности групп является совпадение тензорных типов всех сушественных компонент:

$$
G \approx H \Longleftrightarrow \operatorname{Tor}_{p} G \sim \operatorname{Tor}_{p} H \quad \forall p \in \mathscr{D}\left(G^{0}\right) \cup \mathscr{D}\left(H^{0}\right) u G^{0} \sim H^{0}
$$

ДоКАЗАТЕЛЬСТВо. Прежде всего, заметим, что для монопериодических груп тензорная эквивалентность совпадает с факторной. Поэтому тензорная эквивалентность существенных компонент влечет их факторную эквивалентность, а значит, и факторную эквивалентность их сумм, что в свою очередь влечет изофакторность самих групп. 
Обратно, пусть $G \approx H$. В силу критерия $(0 \otimes p)$-тривиальности тензорное произведение $G \otimes \mathbb{Q}_{p}=0$ в том и только том случае, когда $p$ является делителем 0-компоненты. Следовательно, $\mathscr{D}\left(G^{0}\right)$ является инвариантом тензорного, а значит, и факторного типа. То есть $G \sim H$ влечет $\mathscr{D}\left(G^{0}\right)=\mathscr{D}\left(H^{0}\right)$, но последнее влечет $G^{0} \sim H^{0}$ в силу критерия делителей.

Пусть $p$ является делителем нуль-компонент. Тогда результат тензорного умножения групп $G$ и $H$ на $p$-групшы определяется лишь их $p$-компонентами. Поэтому, тензорно умножая на $\mathbb{Z}_{p}$, мы можем доказать, что $\operatorname{Tor}_{p} G$ имеет ту же делимость, что и $\operatorname{Tor}_{p} H$. А в случае делимости обеих компонент индекс кофакторности с $\mathbb{Z}_{p}$ покажет нам совпадение индексов тривиальности компонент. А это уже влечет их тензорную эквивалентность в силу примарной классификации.

СПЕКТРАЛЬНЫЙ КРИТЕРИЙ ИЗОФАКТОРНОСТИ. Группы факторно әквивалентны в том и только том случае, когда совпадают их факторные спектры.

ДоКАЗАТЕЛЬСТВО. Если $G \sim H$, то $\mathscr{D}(G)=\mathscr{D}(H)$ в силу критерия делимости. Далее, включение $p \in \mathscr{D}\left(G^{0}\right)$ проверяется тензорным умножением на $\mathbb{Q}_{p}$. А именно, из критерия тривиальности для $p$-групп вытекает, что $G \otimes \mathbb{Q}_{p}=0$ если и только если $p \in \mathscr{D}\left(G^{0}\right)$. Поэтому тензорная эквивалентность групп влечет совпадение множеств делителей их 0-компонент. Принадлежность нуля к множеству периодов проверяется умножением на $\mathbb{Q}$. Для доказательства совпадения множеств сушественных периодов потребуется использовать изофакторность групп. А именно, если $p$ является сушественным периодом группы $G$, то наличие кручения $\operatorname{Tor}_{p} G$ проверяется индексом кофакторности $\left(G, \mathbb{Z}_{p}\right)$, который в этом случае должен быть положителен (равняется трем, если $\operatorname{Tor}_{p} G \sim \mathbb{Z}_{p}$, двум, если $\operatorname{Tor}_{p} G \sim \mathbb{Q}_{p}$, и нулю, если $\left.\operatorname{Tor}_{p} G \sim 0\right)$. Поскольку для $H$ мы в этом случае имеем те же индексы, то, зная делимость $H^{0}$ и положительность $\left(H, \mathbb{Z}_{p}\right)$, можем сделать вьвод о наличии $\operatorname{Tor}_{p} H \neq 0$. Таким образом, установлено, что факторные спектры являются инвариантами факторного типа.

Поскольку тензорньй тип монопериодической групш определяется множеством ее делителей, постольку группы с одинаковыми факторными спектрами имеют тензорно эквивалентные сушественные компоненты и являются факторно эквивалентньми в силу компонентного критерия изофакторности. Теорема полностью доказана.

\section{Теорема Бокштейна о базисе}

Для любой группы $G$ и простого числа $p$ определим группу $\sigma_{p}(G)$, назьваемую бокштейновским р-фактором групшы $G$, следующим образом: $\sigma_{p}(G)=\mathbb{Z}_{(p)}$, если $p \notin \mathscr{D}\left(G^{0}\right), \sigma_{p}(G)=\mathbb{Z}_{p}$, если $p \in \mathscr{D}\left(G^{0}\right) \backslash \mathscr{D}(G), \sigma_{p}(G)=\mathbb{Q}_{p}$, если $p \in \mathscr{T}^{0}(G) \cap \mathscr{D}(G)$, и $\sigma_{p}(G)=0$, если $p \in \mathscr{D}(G) \backslash \mathscr{T}(G)$.

И положим $\sigma_{0}(G)=\mathbb{Q}$, если $G^{0} \neq 0$, и $\sigma_{0}(G)=0$, если $G^{0}=0$. Из этого определения, зная поведение факторных спектров при тензорном произведении, нетрудно получить следующее, нужное нам в дальнейшем утверждение.

ЛЕММА О ФАКТОРЕ ЛОКАЛИЗАЦИИ. $\sigma_{p} G=\sigma_{p}\left(G \otimes \mathbb{Z}_{(p)}\right)$.

Следующее тождество нетрудно проверить с помошью спектрального критерия. Именно оно, как мы скоро увидим, и представляет собой основное содержание Первой 
Теоремы Бокштейна. И мы будем назьвать его разложением Бокштейна.

$$
G \approx \bigoplus_{p \in \mathfrak{P}_{0}} \sigma_{p}(G)
$$

Тензорный тип группы $G$ называется неразложимылм, если равенство $G \sim G_{1} \oplus G_{2}$ влечет $G \sim G_{1}$ или $G \sim G_{2}$. Таковы типы, представленные бокштейновскими группами, что нетрудно получить из спектрального критерия. Из доказанной теоремы вытекает, что и наоборот, всякий неразложимьй тензорньй тип является бокштейновским, т.е. является тензорным типом бокштейновской группы.

Неравенства Бокштейна. При том изложении теории Бокштейна, которая приводится в этой статье, по существу, отпадает потребность рассмотрения так называемых неравенств Бокштейна. Однако, они также могут быть естественно рассмотрены и при этом подходе.

Так, полученные результаты дают следующую таблищу сравнений бокштейновских групш:

$$
\mathbb{Q}_{p} \ll \mathbb{Q} \ll \mathbb{Z}_{(p)} \gg \mathbb{Z}_{p} \gg \mathbb{Q}_{p} .
$$

При этом бокштейновские группы различных положительных факторностей между собой несравнимы. Эти сравнения соответствуют следующим четырем неравенствам Бокштейна.

$$
\operatorname{dim}_{\mathbb{Q}_{p}} X \leqslant \operatorname{dim}_{\mathbb{Z}_{p}} X \leqslant \operatorname{dim}_{\mathbb{Z}_{(p)}} X \geqslant \operatorname{dim}_{\mathbb{Q}} X \geqslant \operatorname{dim}_{\mathbb{Q}_{p}} X
$$

(Здесь под $X$ понимается произвольный компакт.)

Для того чтобы сформулировать аналоги остальных двух неравенств Бокштейна для любой групшы $G$, обозначим через $D_{G}$ функиию кофакторности, которая каждой группе $H$ ставит в соответствие индекс кофакторности $D_{G}(H)=(G, H)$. Тогда аналоги двух оставшихся неравенств Бокштейна могут быть сформулированы следующим образом:

$$
D_{\mathbb{Q}_{p}} X \leqslant \max \left\{D_{\mathbb{Q}} X, D_{\mathbb{Z}_{(p)}} X-1\right\}, \quad D_{\mathbb{Z}_{(p)}} X \leqslant \max \left\{D_{\mathbb{Q}} X, D_{\mathbb{Q}_{p}}+1\right\}
$$

(Здесь через $X$ обозначена произвольная группа.)

Они получаются из рассмотрения точной последовательности $0 \rightarrow \mathbb{Z}_{(p)} \rightarrow \mathbb{Q} \rightarrow$ $\mathbb{Q}_{p} \rightarrow 0$ ввиду следуюшего общего утверждения.

НЕРАВЕНСТВА ТОчНОЙ ПОСЛЕДОВАТЕЛЬНОСТИ. Для короткой точной последовательности $0 \rightarrow A \rightarrow B \rightarrow C \rightarrow 0$ справедливь следуюшие неравенства: $D_{B} \leqslant \max \left\{D_{A}, D_{C}\right\}, D_{A} \leqslant \max \left\{D_{B}, D_{C}+1\right\}, D_{C} \leqslant \max \left\{D_{B}, D_{A}-1\right\}$.

Доказательство получается из анализа точной тензорно-периодической последовательности, возникающей при умножении короткой точной последовательности на некоторую группу. Мы докажем здесь только первое неравенство, но зато в следующей более сильной форме:

НЕРАВЕНСТВО СРЕДНЕГО чЛЕНА. Для точной последовательности $A \rightarrow B \rightarrow$ $C \rightarrow D \rightarrow E$ справедливо неравенство $D_{C} \leqslant \max \left\{D_{A}, D_{B}, D_{D}, D_{E}\right\}$. 
ДокАЗАТЕльство. Пусть $G$ - группа, для которой $G \otimes(A \oplus B \oplus D \oplus E)=0$. Пусть $B^{\prime}$ является факторгруппой групшы $B$ по подгруппе, являюшейся образом $A$, и $D^{\prime}$ является факторгруппой группы $D$ по ядру гомоморфизма в $E$. Тогда и $G \otimes\left(B^{\prime} \oplus\right.$ $\left.E^{\prime}\right)=0$. Но мы имеем короткую точную последовательность $0 \rightarrow B^{\prime} \rightarrow C \rightarrow D^{\prime} \rightarrow 0$, из рассмотрения тензорного произведения которой на $G$ получаем, что $G \otimes C=0$. Пусть теперь, кроме того, $G *(A \oplus B \oplus D \oplus E)=0$. Обозначим через $C^{\prime}$ фактор-группу групшы $C$ по подгрупе, которая одновременно является образом гомоморфизма из $B$ и ядром гомоморфизма в $D$. Имеем следуюшие точные последовательности: $0 \rightarrow C^{\prime} \rightarrow$ $D \rightarrow E$ и $0 \rightarrow B \rightarrow C \rightarrow C^{\prime} \rightarrow 0$, из рассмотрения периодических произведений которых на $G$ получаем: из первой - что $G * C^{\prime}=0$, а затем из второй - что $G * C=0$, что и завершает доказательство.

\section{§. Кюннетовские алгебры полигрупп}

\section{Верхняя алгебра Кюннета}

Обозначать полигрупшы мы будем рукописньми латинскими буквами $\mathscr{F}, \mathscr{G}, \mathscr{H}, \ldots$ $n$-й член полигрупnы $\mathscr{F}, \mathscr{G}, \mathscr{H}$ и т. д. обозначается соответственно $F_{n}, G_{n}, H_{n}$ и т. д. Номер члена полигрупшы назьвается также его размерностью. Bыссотой полигруппы $\mathscr{G}$ будем назьвать максимальное $n$, для которого $G_{n} \neq 0$, обозначение: alt $\mathscr{G}$.

Будем говорить, что полигруппа $\left\{G_{0}, G_{1}, \ldots, G_{n}, \ldots\right\}$ имеет сильное начало, если $G_{0} \approx \mathbb{Z}$, Tor $G_{0}=0=\operatorname{Tor} G_{1}$ и или $G_{1} \approx \mathbb{Z}$, или $G_{1}=0$.

Полигруппы конечной высоты с сильным началом называются верхними.

Суммой полигрупп $\mathscr{G}_{\text {и }} \mathscr{H}$ назьваетсяполигруппа $\left\{G_{n} \oplus H_{n}\right\}$; обозначение: $\mathscr{G} \oplus \mathscr{H}$. Также почленно определяется операция бесконечной суммы полигруп. Операция суммы сохраняет начальные условия, и потому сумма верхних полигрупп является верхней. Верхним произведением Кюннета полигруп $\mathscr{G}$ и $\mathscr{H}$ назьвается полигруппа, обозначаемая $\mathscr{G}[\times] \mathscr{H}, n$-й член которой задается формулой

$$
\bigoplus_{i+j=n}\left(G_{i} \otimes H_{j}\right) \oplus \bigoplus_{i+j=n+1}\left(G_{i} * H_{j}\right) .
$$

Мы в дальнейшем будем обычно опускать знак кюннетовского произведения, и писать $\mathscr{G} \mathscr{H}$ вместо $\mathscr{G}[\times] \mathscr{H}$, если это не приводит к недоразумениям.

Верхнее произведение Кюннета сохраняет конечность и сильное начало. Для верх-

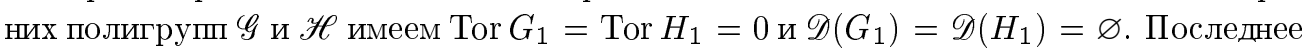
условие, как мы знаем, характеризует тензорньй тип $\mathbb{Z}$ среди групп без кручения. По формулам сложения периодов и делителей оба эти свойства сохранятся для произведения $G_{1} \otimes H_{1}$, которое как раз совпадает с первьм членом произведения $\mathscr{G} \mathscr{H}$. И начальньй член произведения $\mathscr{G} \mathscr{H}$, равньй $G_{0} \otimes H_{0}$, имеет тензорньй тип $\mathbb{Z}$ и не имеет кручения. Таким образом, на множестве верхних полигрупп определены операции сложения и умножения.

Из свойств дистрибутивности тензорного и периодического произведений непосредственно вытекает свойство дистрибутивности полигруппового произведения по отношению к полигрупповой сумме:

$$
\mathscr{G} \bigoplus_{i \in I} \mathscr{H}_{i}=\bigoplus_{i \in I} \mathscr{G}_{i}
$$


Кроме того, ясно, что полигрупповые операции сложения и умножения коммутативны и ассоциативны. Непосредственно из определения вытекают такие оценки для высоты произведения и суммы полигрупп:

$$
\begin{gathered}
\operatorname{alt}(\mathscr{G} \oplus \mathscr{H})=\max \{\text { alt } \mathscr{G}, \text { alt } \mathscr{H}\} \\
\max \{\operatorname{alt} \mathscr{G}, \text { alt } \mathscr{H}\}+1 \leqslant \operatorname{alt} \mathscr{G} \mathscr{H} \leqslant \text { alt } \mathscr{G}+\text { alt } \mathscr{H} .
\end{gathered}
$$

Назовем верхние полигрупшы $\mathscr{G}$ и $\mathscr{H}$ взаимно простыми, если $\left(G_{n}, H_{k}\right)=0$ для любых $n, k>1$. Для взаимно простых полигрупп высота произведения совпадает с нижней оценкой.

Высотность. Будем говорить, что верхние полигрупшы $\mathscr{G}$ и $\mathscr{H}$ имеют один и тот же же высотныи тип, одинаковую высотность или равновысотны, и писать ALT $\mathscr{G}=\operatorname{ALT} \mathscr{H}$, если alt $\mathscr{F} \mathscr{H}=$ alt $\mathscr{F} \mathscr{G}$ для любой верхней полигрупшы $\mathscr{F}$. Неравенство $\mathrm{ALT} \mathscr{G} \geqslant \mathrm{ALT} \mathscr{H}$ означает, что alt $\mathscr{F} \mathscr{G} \geqslant$ alt $\mathscr{F} \mathscr{H}$ для любой верхней полигрупшы $\mathscr{F}$.

Полигрупповые операции суммы и произведения индуцируют операции суммы и произведения на множестве высотных типов верхних полигрупп, что доказьвается так же, как аналогичное утверждение для тензорных типов.

Для групшы $G$ и целого $n \geqslant 0$ через $G^{(n)}$ обозначаем полигруппу, у которой тривиальны все члены, за исключением $n$-го, которьй совпадает с $G$.

Всякая верхняя полигруппа нулевой высоты имеет высотньй тип полигруппы $\mathbb{Z}^{(0)}=\{\mathbb{Z}, 0,0, \ldots\}$, которая представляет собой наименьший высотньй тип. Эта полигруппа ведет себя, как единичньй элемент, при умножении Кюннета. Bсе верхние полигрупш единичной высоты имеют высотный тип полигруппы $\mathbb{Z}^{(0)} \oplus \mathbb{Z}^{(1)}=\{\mathbb{Z}, \mathbb{Z}, 0,0, \ldots\}$. Полигрупшы высоты $\leqslant 1$ мы будем назьвать тривиальными. Наибольший высотный тип представляет стационарная полигрупа $\mathbb{Z}^{(\infty)}=\{\mathbb{Z}, \mathbb{Z}, \mathbb{Z}, \mathbb{Z}, \ldots\}$. Любое ограниченное множество высотных типов имеет точную верхнюю грань, которая представляется суммой представляюших типы полигрупп. Отсюда ввиду наличия наименьшего элемента вытекает, что любое множество высотных типов имеет точную нижнюю грань. Таким образом, множество высотных типов является решеткой. Операция суммы совпадает с операцией взятия верхней грани и не несет дополнительной информации о его структуре. Дистрибутивность умножения Кюннета по отношению к сумме влечет согласованность умножения с порядком. То есть неравенство ALT $\mathscr{G} \geqslant \operatorname{ALT} \mathscr{H}$ влечет ALT $\mathscr{G} \mathscr{F} \geqslant \operatorname{ALT} \mathscr{H} \mathscr{F}$ для любой полигруппы $\mathscr{F}$. Множество высотных типов верхних полигруп обозначаем $\mathfrak{K}^{*}$ и назьваем верхней алгеброй Кюннета. Она является решеточной полугруппой по отношению к описанному порядку и операции верхнего произведения Кюннета. Впредь по умолчанию мы будем считать, что рассматриваемые полигрупшы верхние.

Верхние моногруппы. Верхняя полигрупа, у которой тривиальны все члены размерности $>1$, за исключением, может быть, одного, назьвается верхней моногруппой. Для группы $G$ и натурального $n>1$ через $G^{[n]}$ обозначим верхнюю моногруппу $\mathbb{Z}^{(0)} \oplus \mathbb{Z}^{(1)} \oplus G^{(n)}, n$-й член которой совпадает с $G$, а все остальные члены размерности $>1$ тривиальны. Таким образом, alt $G^{[n]}=n$ для нетривиальной $G$.

Группа $G_{\text {alt }} \mathscr{G}$ назьвается высшей групой полигруппы $\mathscr{G}$ и обозначается $|\mathscr{G}|$.

Следуюшее предложение раскрывает связь между изофакторностью и равновысотностью. 
КРИТЕРИЙ РАВНОВЫСОТНОСТИ МОНОГРУПП. Верхние моногруппы $\mathscr{G}, \mathscr{H}$ равновиссотны $\mathrm{ALT} \mathscr{G}=\mathrm{ALT} \mathscr{H}$, если и только если alt $\mathscr{G}=$ alt $\mathscr{H}$ и $|\mathscr{G}| \approx|\mathscr{H}|$.

ДоКАЗАТЕЛЬСТВО. Необходимость условия совпадения высот полигрупш очевидна. Что касается изофакторности, то при умножении моногруппы $\mathscr{G}$ на моногруппу $\mathscr{F}$ высота произведения $\mathscr{G} \mathscr{F}$ определяется на основе индекса кофакторности $(|\mathscr{G}|,|\mathscr{F}|)$. А именно, она равна alt $\mathscr{F}+\operatorname{alt} \mathscr{G}$, если $(|\mathscr{G}|,|\mathscr{F}|)=3$, она равна alt $\mathscr{F}+$ alt $\mathscr{G}-1$, если $(|\mathscr{G}|,|\mathscr{F}|)=2$, и она равна $\max \{$ alt $\mathscr{F}$, alt $\mathscr{G}\}+1$, если $(|\mathscr{G}|,|\mathscr{F}|)=0$.

Моногрупша $|\mathscr{G}|^{[\text {alt } \mathscr{G}]}$ назьвается высшей моногруппой полигрупшы $\mathscr{G}$ и обозначается $\lceil\mathscr{G}\rceil$.

Полигруппы $\mathscr{G}$ и $\mathscr{H}$ будем назьвать почленно изофакторныцми и писать $\mathscr{G} \approx \mathscr{H}$, если $G_{n} \approx H_{n}$ для любого $n$.

ФАКТОРНАЯ ОПРЕДЕЛЕННОСТЬ ВЫСОТНОСТИ. Если $\mathscr{G} \approx \mathscr{H}$, mo ALT $\mathscr{G}=$ ALT $\mathscr{H}$.

ДокАЗАТЕЛЬСТвО. Ясно, что почленная изофакторность полигрупा влечет равенство их высот. Предположим, что для полигрупш высоты $<n$ доказано, что почленная изофакторность влечет равновысотность. Пусть $\mathscr{G}$ и $\mathscr{H}$ имеют высоту $n$. Пусть $\mathscr{G}^{\prime}$ и $\mathscr{H}^{\prime}-$ усеченные полигрупшы высоты $n-1$, для которых $G_{k}^{\prime}=G_{k}$ и $H_{k}^{\prime}=H_{k}$ при $k<n$. Тогда $\mathscr{G}^{\prime} \approx \mathscr{H}^{\prime}$ и по предположению индукции $\operatorname{ALT} \mathscr{G}^{\prime}=$ ALT $\mathscr{H}^{\prime}$. По критерию равновысотности высшие моногрупшы у $\mathscr{G}$ и $\mathscr{H}$ равновысотны: $\mathrm{ALT}\lceil\mathscr{G}\rceil=\operatorname{ALT}\lceil\mathscr{H}\rceil$. Но $\mathscr{G}=\mathscr{G}^{\prime} \oplus\lceil\mathscr{G}\rceil$ и $\mathscr{H}=\mathscr{H}^{\prime} \oplus\lceil\mathscr{H}\rceil$, откуда и следует, что $\operatorname{ALT} \mathscr{G}=\operatorname{ALT} \mathscr{H}$

Отметим такое следствие доказанного:

МОНОГРУППОВОЕ РАЗЛОЖЕНИЕ. Всякая полигруппа $\mathscr{G}$ имеет высотный тип суммы моногрупn: $\operatorname{ALT} \mathscr{G}=\operatorname{ALT} \bigoplus_{n \leqslant \text { alt } \mathscr{G}} G_{n}^{[n]}$.

Две моногруппы $\mathscr{G}$ и $\mathscr{G}^{\prime}$ будем называть $с$ мещеннылм друг относительно друга, если $|\mathscr{G}|=\left|\mathscr{G}^{\prime}\right|$. При этом мы говорим что $\mathscr{G}$ смещена вверх (соответственно вниз) относительно $\mathscr{G}^{\prime}$, если alt $\mathscr{G}>$ alt $\mathscr{G}^{\prime}$ (соответственно alt $\left.\mathscr{G}<\operatorname{alt} \mathscr{G}^{\prime}\right)$.

ФоРмУЛЫ СМЕЩЕнИЯ. Пусть $\mathscr{G}$ - произвольная полигруппа, $\mathscr{B}^{\prime}$ - моногруппа, смещенная вверх относительно моногруппь $\mathscr{B}$. Тогда справедливо неравенство смешения alt $\mathscr{G} \mathscr{B}^{\prime}-\operatorname{alt} \mathscr{G} \mathscr{B} \leqslant \operatorname{alt} \mathscr{B}^{\prime}-\operatorname{alt} \mathscr{B} ;$ ссли жеe alt $\mathscr{B} \mathscr{G}>$ alt $\mathscr{G}+1$ или alt $\mathscr{B} \geqslant$ alt $\mathscr{G}$, то имеет место равенство смещения alt $\mathscr{G} \mathscr{B}^{\prime}-\operatorname{alt} \mathscr{G} \mathscr{B}=\operatorname{alt} \mathscr{B}^{\prime}-\operatorname{alt} \mathscr{B}$.

ДоКАЗАТЕЛЬСТво. Если $\left(|\mathscr{B}|, G_{n}\right)=0$ при любом $n>1$, то $\mathscr{B}$ и $\mathscr{G}$ взаимно просты, так же как $\mathscr{B}^{\prime}$ и $\mathscr{G}$. Тогда alt $\mathscr{B} \mathscr{G}=1+\max \{$ alt $\mathscr{B}$, alt $\mathscr{G}\}$ и alt $\mathscr{B}^{\prime} \mathscr{G}=1+$ $\max \left\{\right.$ alt $\mathscr{B}^{\prime}$, alt $\left.\mathscr{G}\right\}$, и справедливость неравенства смешения очевидна. Если же alt $\mathscr{B} \mathscr{G}>$ alt $\mathscr{G}+1$ или alt $\mathscr{B} \geqslant$ alt $\mathscr{G}$, то получаем alt $\mathscr{B}^{\prime} \mathscr{G}=1+$ alt $\mathscr{B}^{\prime}$ и alt $\mathscr{B}^{\prime} \mathscr{G}=1+$ alt $\mathscr{B}^{\prime}$, поэтому справедливо равенство смешения.

В противном случае пусть $k=\max \left\{i+\left(G_{i},|\mathscr{B}|\right)-3 \mid\left(G_{i},|\mathscr{B}|\right)>0\right\}$. Тогда alt $\mathscr{B} \mathscr{G}=\max \{$ alt $\mathscr{B}+k$, alt $\mathscr{G}+1\}$ и alt $\mathscr{B}^{\prime} \mathscr{G}=\max \left\{\right.$ alt $\mathscr{B}^{\prime}+k$, alt $\left.\mathscr{G}+1\right\}$. Из этих формул очевидно неравенство смещения. А выполнение условий alt $\mathscr{B} \mathscr{G}>$ alt $\mathscr{G}+1$ или alt $\mathscr{B} \geqslant$ alt $\mathscr{G}$ приводит к формулам alt $\mathscr{B} \mathscr{G}=$ alt $\mathscr{B}+k$ и alt $\mathscr{B}^{\prime} \mathscr{G}=$ alt $\mathscr{B}^{\prime}+k$, из которых вытекает равенство смешения. 
Моногруппа $\mathscr{G}$ назьвается бокштейновской, если ее высшая групп $|\mathscr{G}|$ принадлежит системе Бокштейна.

ЛЕМмА О ТЕСТИРОВАНИИ. Пусть $\mathscr{G}$ и $\mathscr{H}$ являются полигруппами высот $\leqslant n$ $u \operatorname{ALT} \mathscr{G} \neq \operatorname{ALT} \mathscr{H} . \quad$ Тогда alt $\mathscr{G} \mathscr{B} \neq$ alt $\mathscr{H} \mathscr{B}$ для некоторой бокштейновской моногруппьи $\mathscr{B}$ выссоты $n$.

ДокАЗАТЕльство. Рассмотрим сначала случай, когда alt $\mathscr{G}>$ alt $\mathscr{H}$. Тогда

$$
\operatorname{alt} \mathscr{G} \mathbb{Z}^{[n]}=\operatorname{alt} \mathscr{G}+n>\text { alt } \mathscr{H}+n=\operatorname{alt} \mathscr{H} \mathbb{Z}^{[n]}
$$

Поскольку $\mathbb{Z}$ изофакторна сумме $\bigoplus \mathbb{Z}_{(p)}$, постольку для некоторого $p$ имеем $|\mathscr{G}| \otimes$ $\mathbb{Z}_{(p)} \neq 0$. Тогда

$$
\operatorname{alt} \mathscr{G} \mathbb{Z}_{(p)}^{[n]}=\operatorname{alt} \mathscr{G}+n>\text { alt } \mathscr{H}+n \geqslant \operatorname{alt} \mathscr{H} \mathbb{Z}_{(p)}^{[n]},
$$

т.е. $\mathbb{Z}_{(p)}^{[n]}$ является искомой бокштейновской моногруппой. Аналогично разбирается случай alt $\mathscr{G}<$ alt $\mathscr{H}$. Пусть теперь alt $\mathscr{G}=$ alt $\mathscr{H}$. Если ALT $\mathscr{G} \neq$ ALT $\mathscr{H}$, то для некоторой полигруппы $\mathscr{F}$ будет alt $\mathscr{G} \mathscr{F} \neq$ alt $\mathscr{H} \mathscr{F}$. Поскольку $\mathscr{F}$ имеет высотность суммы моногрупп, постольку для одного из слагаемых этой суммы, которое мы обозначим $\mathscr{F}^{\prime}$, также имеем неравенство alt $\mathscr{G} \mathscr{F}^{\prime} \neq$ alt $\mathscr{H} \mathscr{F}^{\prime}$. Но $\left|\mathscr{F}^{\prime}\right|$ изофакторна сумме бокштейновских груп, поэтому моногруппа $\mathscr{F}^{\prime}$ также изофакторна сумме бокштейновских моногрупп. Тогда для одной из этих бокштейновских моногруп, обозначаемой $\mathscr{B}^{\prime}$, также имеем неравенство alt $\mathscr{G} \mathscr{B}^{\prime} \neq$ alt $\mathscr{H} \mathscr{B}^{\prime}$. Пусть $\mathscr{B}$ - моногруппа высоты $n$, смешенная относительно $\mathscr{B}^{\prime}$. Если alt $\mathscr{B}^{\prime}>n$, то вьполнено равенство смещения, и мы получим, что alt $\mathscr{G} \mathscr{B} \neq$ alt $\mathscr{H} \mathscr{B}$, что нам и требуется. Пусть теперь alt $\mathscr{B}^{\prime}<n$, и предположим, что alt $\mathscr{G} \mathscr{B}^{\prime}>$ alt $\mathscr{H} \mathscr{B}^{\prime}$. Поскольку alt $\mathscr{H} \mathscr{B}^{\prime} \geqslant$ alt $\mathscr{H}+1=\operatorname{alt} \mathscr{G}+1$, постольку alt $\mathscr{G} \mathscr{B}^{\prime}>$ alt $\mathscr{G}+1$, и равенство смешения для $\mathscr{G}$ дает alt $\mathscr{B} \mathscr{G}=$ alt $\mathscr{B}^{\prime} \mathscr{G}+$ alt $\mathscr{B}-$ alt $\mathscr{B}^{\prime}$. A для $\mathscr{H}$ на основании неравенства смешения имеем alt $\mathscr{B} \mathscr{H} \leqslant$ alt $\mathscr{B}^{\prime} \mathscr{H}+$ alt $\mathscr{B}$ - alt $\mathscr{B}^{\prime}$. Откуда alt $\mathscr{B} \mathscr{G}>$ alt $\mathscr{B} \mathscr{H}$. Что и завершает доказательство.

Бокштейновские моногруппы одинаковой факторности, как нетрудно видеть, имеют сравнимые высотности, и справедлив следующий

КРИТЕРИЙ СРАВНЕНИЯ БОКШТЕЙНОВСКИХ МОНОГРУПП. Если $\mathscr{B} u \mathscr{B}^{\prime}-\partial \theta e$ бокштейновские моногруппь одинаковой факторности $u$ alt $\mathscr{B}>$ alt $\mathscr{B}^{\prime}$, то $\operatorname{ALT} \mathscr{B}>\operatorname{ALT} \mathscr{B}^{\prime} ;$ если же alt $\mathscr{B}=$ alt $\mathscr{B}^{\prime}$, то неравенство ALT $\mathscr{B} \geqslant \mathrm{ALT} \mathscr{B} \prime$ равносильно факторному доминированию $|\mathscr{B}| \gg\left|\mathscr{B}^{\prime}\right|$.

\section{Факторы Бокштейна}

Для $p$ - простого или нуля, определим полигрупповой р-фактор Бокштейна полигруппы $\mathscr{G}=\left\{G_{n}\right\}$ как полигрупу $\Sigma_{p} \mathscr{G}, n$-й член которой при $n>1$ совпадает с $\sigma_{p} G_{n}$ а начальные члены равны $\mathbb{Z}$. Разложение Бокштейна для групп влечет за собой разложение для полигрупп

$$
\mathscr{G} \approx \bigoplus_{p \in \mathfrak{P}_{0}} \Sigma_{p} \mathscr{G}
$$


Очевидно также следующее свойство аддитивности полигруппового фактора Бокштейна:

$$
\Sigma_{p}(\mathscr{G} \oplus \mathscr{H}) \approx \Sigma_{p} \mathscr{G} \oplus \Sigma_{p} \mathscr{H}
$$

Определим теперь моногрупповой р-фактор Бокштейна $\sigma_{p} \mathscr{G}$ полигрупшы $\mathscr{G}$ как высшую моногруппу $\sigma_{p} \mathscr{G}=\left\lceil\Sigma_{p} \mathscr{G}\right\rceil$.

Полигруппу $\mathscr{G}$ назовем гомофакторной факторности $p$, если $\mathscr{G} \approx \Sigma_{p} \mathscr{G}$ для некоторого $p$. Очевидно, что сумма и произведение гомофакторных полигрупп одинаковой факторности также гомофакторно. Из критерия сравнения бокштейновских моногрупп немедленно вытекает следуюший

ГОМОФАКТОРНЫЙ КРИТЕРИЙ СРАВНЕНИЯ. Если $\mathscr{G}$ и $\mathscr{H}$ - гомофакторные полигруппь одинаковой факторности, то $\mathrm{ALT} \mathscr{G} \geqslant \mathrm{ALT} \mathscr{H}$, если и только если $\operatorname{ALT}\lceil\mathscr{G}\rceil \geqslant \operatorname{ALT}\lceil\mathscr{H}\rceil$.

Из этого критерия мы, в частности, получаем, что $\mathrm{ALT} \sigma_{p} \mathscr{G}=\operatorname{ALT} \Sigma_{p} \mathscr{G}$ для любой полигрупшы $\mathscr{G}$, откуда немедленно следует такой факт.

МОНОГРУППОВОЕ РАЗЛОЖЕНИЕ БОКШТЕЙНА. ALT $\mathscr{G}=\operatorname{ALT} \bigoplus_{p \geqslant 0} \sigma_{p} \mathscr{G}$.

Из критерия равновысотности для моногруп и гомофакторного критерия сравнения получаем такое следствие.

ГОМОФАКТОРНЫЙ КРИТЕРИЙ СОВПАДЕНИЯ. Для гомофакторныx nолигрупn $\mathscr{G}$ $\mathscr{H}$ равенство $\mathrm{ALT} \mathscr{G}=\mathrm{ALT} \mathscr{H}$ равносильно совпадению факторов $\sigma_{p} \mathscr{G}=\sigma_{p} \mathscr{H}$.

Из отмеченной выше сравнимости высотных типов бокштейновских моногрупп и гомофакторного критерия сравнения получаем, что гомофакторные полигрупшы одинаковой факторности также имеют сравнимые высотности, откуда получаем такое

ПрАвИЛО ДОмИНИРовАНИЯ. Для гомофакторных полигрупп $\mathscr{G}, \mathscr{H}$ факторности р бокштейновский р-фактор суммы $\sigma_{p}(\mathscr{G} \oplus \mathscr{H})$ совпадает с р-фактором слагаемого большей высотности.

Для любых полигрупп из приведенных выше результатов вытекает такое свойство аддитивности $p$-факторов. Вкупе с приведенньм выше правилом доминирования оно позволяет эффективно определять факторы суммы полигруп.

ФОРМУЛА СЛОЖЕНИЯ ФАКТОРОВ. Для любых полигрупп $\mathscr{G}, \mathscr{H}$ выполнено равенство $\sigma_{p}(\mathscr{G} \oplus \mathscr{H})=\sigma_{p}\left(\sigma_{p} \mathscr{G} \oplus \sigma_{p} \mathscr{H}\right)$.

Полигрупповой $p$-фактор бокштейновской полигруппы факторности $q$, как легко понять, тривиален, если $q \neq p>0$. В случае же $p=0$ это, вообше говоря, не так. В этом случае имеется лишш такое соотношение:

РАЦИОНАЛЬНОЕ НЕРАВЕНСТВо. alt $\sigma_{0}\left(\sigma_{p} \mathscr{G}\right) \leqslant \operatorname{alt} \sigma_{0} \mathscr{G}$.

ДокАЗАТЕЛЬСтво. Заметим, что группа $G_{n}$ при любом $n$ факторно доминирует $\sigma_{p} G_{n}$, поэтому тривиальность $\mathbb{Q} \otimes G_{n}$ влечет тривиальность $\mathbb{Q} \otimes \sigma_{p} G_{n}$, а высота alt $\sigma_{0} \mathscr{G}$ - это наибольшее $n$, для которого нетривиально $\mathbb{Q} \otimes G_{n}$.

Итак, высотность полигруппы определяется ее моногрупповыми факторами. Обратное верно для надрациональных факторов, каковыми мы называем $p$-факторы, для 
которых alt $\sigma_{p} \mathscr{G} \geqslant$ alt $\sigma_{0} \mathscr{G}$, и, вообще говоря, неверно для метарациональных факторов, каковыми мы называем $p$-факторы, для которых alt $\sigma_{p} \mathscr{G}<$ alt $\sigma_{0} \mathscr{G}$. Естественное предположение, что равновысотность полигрупп в свою очередь влечет совпадение моногрупповых факторов Бокштейна, опровергается следуюшим примером.

МЕТАРАЦИОНАЛЬНАЯ АЛЬТЕРНАТИВА. Для любого $k \geqslant 0$ справедливо равенcmво $\operatorname{ALT}\left(\mathbb{Q}^{[n]} \oplus \mathbb{Z}_{(p)}^{[n-k-1]}\right)=\operatorname{ALT}\left(\mathbb{Q}^{[n]} \oplus \mathbb{Q}_{p}^{[n-k]}\right)$.

ДокАЗАТЕльство. Действительно, для непериодических групп $G$ нетривиально произведение $G \otimes \mathbb{Q}$, и потому высота произведения моногруппы $G^{[m]}$ на любую из рассматриваемых будет равна $n+m$. Если же $G=\operatorname{Tor} G$, то $\left(G, \mathbb{Z}_{(p)}\right)=\left(G, \mathbb{Q}_{p}\right)+1$, откуда следует, что $\operatorname{alt}\left(G^{[m]} \mathbb{Z}_{(p)}^{[k]}\right)=\operatorname{alt}\left(G^{[m]} \mathbb{Q}_{p}^{[k]}\right)+1$. И высоты произведений обеих рассматриваемых групп на $G^{[m]}$ равны в этом случае $m+n+k-1$.

Унифицированные факторы. Определим унифицированный моногрупповой $p$-фактор $\sigma_{p}^{+} \mathscr{G}$ как $\sigma_{p} \mathscr{G}$ в случае, если $\left|\sigma_{p} \mathscr{G}\right| \neq \mathbb{Z}_{(p)}$ или alt $\sigma_{p} \mathscr{G} \geqslant \operatorname{alt} \sigma_{0} \mathscr{G}$, и как $\mathbb{Q}_{p}^{\left[\text {alt } \sigma_{p} \mathscr{G}+1\right]}$ в противном случае. В частности, всегда верно $\sigma_{0}^{+} \mathscr{G}=\sigma_{0} \mathscr{G}$. Отметим такое свойство унифищированного фактора, непосредственно вытекающее из его определения.

УНИРАцИОНАЛЬНОсть. Если $\left|\sigma_{p}^{+} \mathscr{G}\right|=\mathbb{Z}_{(p)}, m o$ alt $\sigma_{p}^{+} \mathscr{G}=$ alt $\sigma_{0} \mathscr{G}$.

Из леммы о метарациональной альтернативе следует, что $\operatorname{ALT}\left(\sigma_{p}^{+} \mathscr{G} \oplus \sigma_{0} \mathscr{G}\right)=$ $\operatorname{ALT}\left(\sigma_{p} \mathscr{G} \oplus \sigma_{0} \mathscr{G}\right)$. Поэтому

$$
\operatorname{ALT} \bigoplus_{p \geqslant 0} \sigma_{p} \mathscr{G}=\operatorname{ALT} \bigoplus_{p \geqslant 0} \sigma_{p}^{+} \mathscr{G}=\operatorname{ALT} \mathscr{G} .
$$

Для доказательства инвариантности унифицированных факторов нам потребуется следуюшая таблица умножения на бокштейновские моногрупшы.

ФоРмУЛЫ УМНОЖЕНИЯ. Для любой полигруппь $\mathscr{G}$ высоты $\leqslant n$ справедливь следующие формуль:

(1) $\operatorname{alt} \mathscr{G} \mathbb{Q}^{[n]}=\operatorname{alt} \sigma_{0} \mathscr{G}+n$;

(2) alt $\mathscr{G} \mathbb{Q}_{p}^{[n]}=\operatorname{alt} \sigma_{p} \mathscr{G}+n+\left(\mathbb{Q}_{p},\left|\sigma_{p} \mathscr{G}\right|\right)-3$;

(3) alt $\mathscr{G} \mathbb{Z}_{p}^{[n]}=\operatorname{alt} \sigma_{p} \mathscr{G}+n+\left(\mathbb{Z}_{p},\left|\sigma_{p} \mathscr{G}\right|\right)-3$;

(4) $\operatorname{alt} \mathscr{G} \mathbb{Z}_{(p)}^{[n]}=\max \left\{\right.$ alt $\sigma_{0} \mathscr{G}+n$, alt $\left.\sigma_{p} \mathscr{G}+n\right\}$.

ДокАЗАТЕЛЬСтво. Действительно, в силу изофакторности группы сумме своих бокштейновских факторов можно считать, что $\mathscr{G}=\bigoplus \Sigma_{p} \mathscr{G}$. Пусть $\mathscr{B}$ равна либо $\mathbb{Q}_{p}^{[n]}$, либо $\mathbb{Z}_{p}^{[n]}$. Тогда $\mathscr{B}$ взаимно проста с $\Sigma_{q} \mathscr{G}$ при $q \neq p$, и потому alt $\mathscr{B} \mathscr{G}=\operatorname{alt} \mathscr{B} \Sigma_{p} \mathscr{G}$. Последнее же совпадает с $n+\left(\mathscr{B},\left|\sigma_{p} \mathscr{G}\right|\right)-3$, что и доказывает наше утверждение во втором и третьем случаях.

Моногруппа $\mathbb{Z}_{(p)}^{[n]}$ не взаимно проста с $\Sigma_{0} \mathscr{G}$ и $\Sigma_{p} \mathscr{G}$, если только последние не тривиальны, и в этом случае $\mathbb{Z}_{(p)} \otimes\left|\sigma_{p} \mathscr{G}\right| \neq 0$, так же как $\mathbb{Z}_{(p)} \otimes\left|\sigma_{0} \mathscr{G}\right| \neq 0$. Откуда вытекает четвертьй случай.

Первьй оставляем читателю. 
ИНВАРИАНТНОСТЬ УНИФИЦИРОВАННЫХ ФАКТОРОВ. Если АLT $\mathscr{G}=\operatorname{ALT} \mathscr{H}, m o$ $\sigma_{p}^{+} \mathscr{G}=\sigma_{p}^{+} \mathscr{H}$ для любого простого $p \geqslant 0$.

ДокАЗАТЕЛЬСТво. Пусть alt $\mathscr{G}=$ alt $\mathscr{H}=n$. Если ALT $\mathscr{G}=$ ALT $\mathscr{H}$, то умножая на $\mathbb{Q}^{[n]}$ полигруппы $\mathscr{G}$ и $\mathscr{H}$, получаем полигруппы одинаковых высот, откуда alt $\sigma_{0} \mathscr{G}=$ alt $\sigma_{0} \mathscr{H}$ в силу первой формулы умножения.

Если alt $\sigma_{p} \mathscr{G} \leqslant$ alt $\sigma_{0} \mathscr{G}$, то знание высот произведений полигруппы $\mathscr{G}$ на бокштейновские моногрупшы $\mathbb{Q}_{p}^{[n]}, \mathbb{Z}_{p}^{[n]}, \mathbb{Z}_{(p)}^{[n]}$ позволяет определить, основьваясь на четвертой формуле умножения, высоту alt $\sigma_{p} \mathscr{G}$, зная которую можно определить индексы кофакторности группы $\left|\sigma_{p} \mathscr{G}\right|$ с группами $\mathbb{Z}_{p}$ и $\mathbb{Q}_{p}$, что позволяет определить саму групу $\left|\sigma_{p} \mathscr{G}\right|$. То же самое относится и к $\mathscr{H}$, а поскольку высоты произведений полигрупшы $\mathscr{H}$ с бокштейновскими моногруппами такие же самые, то и результаты для $\mathscr{H}$ получатся те же. То есть alt $\sigma_{p} \mathscr{H}=$ alt $\sigma_{p} \mathscr{\mathscr { G }}$ и $\left|\sigma_{p} \mathscr{H}\right|=\left|\sigma_{p} \mathscr{G}\right|$. А поскольку в этом случае унифицированньй фактор совпадает с обычным, те же результаты справедливы и для унифицированного фактора.

Рассмотрим теперь метарациональный случай, когда alt $\sigma_{p} \mathscr{G}>$ alt $\sigma_{0} \mathscr{G}$. Если alt $\mathscr{G} \mathbb{Q}_{p}^{[n]} \neq$ alt $\mathscr{Z}_{p}^{[n]}$, то и alt $\mathscr{H} \mathbb{Q}_{p}^{[n]} \neq$ alt $\mathscr{H} \mathbb{Z}_{p}^{[n]}$, в этом случае $\sigma_{p} \mathscr{G}=\sigma_{p} \mathscr{H}=\mathbb{Z}_{p}$, и в силу третьей формулы умножения мы сможем получить, что alt $\sigma_{p} \mathscr{G}=\operatorname{alt} \sigma_{p}^{+} \mathscr{G}=$ alt $\sigma_{p} \mathscr{H}=\operatorname{alt} \sigma_{p}^{+} \mathscr{H}=\operatorname{alt} \mathscr{G} \mathbb{Z}_{p}^{[n]}-n$.

Наконец, если alt $\mathscr{G}_{\mathbb{Q}_{p}^{[n]}}^{[n]}=\operatorname{alt} \mathscr{G} \mathbb{Z}_{p}^{[n]}=\operatorname{alt} \mathscr{H} \mathbb{Q}_{p}^{[n]}=\operatorname{alt} \mathscr{H} \mathbb{Z}_{p}^{[n]}$ и $\left|\sigma_{p} \mathscr{G}\right|=\left|\sigma_{p} \mathscr{H}\right|$, то совпадение высот alt $\sigma_{p} \mathscr{G}=$ alt $\sigma_{p} \mathscr{H}$ следует из формул умножения, и отсюда в свою очередь вытекает совпадение унифицированных факторов $\left|\sigma_{p}^{+} \mathscr{G}\right|=\left|\sigma_{p}^{+} \mathscr{H}\right|$. Если же $\left|\sigma_{p} \mathscr{G}\right| \neq\left|\sigma_{p} \mathscr{H}\right|$, то один из них, допустим, первый, совпадает с $\mathbb{Z}_{(p)}$, а второй в таком случае совпадает с $\mathbb{Q}_{p}$. Формулы умножения позволяют в этом случае получить, что alt $\sigma_{p} \mathscr{G}=$ alt $\sigma_{p} \mathscr{H}-1$. Но тогда как раз получится, что alt $\sigma_{p}^{+} \mathscr{H}=$ alt $\sigma_{p} \mathscr{G}$. А поскольку в этом случае $\sigma_{p}^{+} \mathscr{G}=\sigma_{p} \mathscr{G}$, постольку получаем оба требуемых равенства $\left|\sigma_{p}^{+} \mathscr{G}\right|=\left|\sigma_{p}^{+} \mathscr{H}\right|$ и alt $\sigma_{p}^{+} \mathscr{G}=$ alt $\sigma_{p}^{+} \mathscr{H}$.

Из только что доказанной инвариантности унифицированных факторов и отмеченной изотипности полигрупшы сумме своих унифицированных факторов Бокштейна вытекает такое следствие.

ФАКТОРНЫЙ КРИТЕРИЙ СРАВНЕНИЯ. Неравенство АLT $\mathscr{G} \geqslant$ ALT $\mathscr{H}$ равносильно системе неравенств ALT $\sigma_{p}^{+} \mathscr{G} \geqslant \operatorname{ALT} \sigma_{p}^{+} \mathscr{H}, p \geqslant 0$. Равенство ALT $\mathscr{G}=$ ALT $\mathscr{H}$ равносильно совпадению всех унифицированных р-факторов $\sigma_{p}^{+} \mathscr{G}=\sigma_{p}^{+} \mathscr{H}$.

ДоКАЗАТЕЛЬСТВО. В доказательстве нуждается лишь то, что неравенство типов $\mathrm{ALT} \mathscr{G} \geqslant \mathrm{ALT} \mathscr{H}$ влечет неравенство типов факторов $\mathrm{ALT} \sigma_{p}^{+} \mathscr{G} \geqslant \mathrm{ALT} \sigma_{p}^{+} \mathscr{H}$. Поскольку в этом случае $\operatorname{ALT} \mathscr{G}=\operatorname{ALT}(\mathscr{G} \oplus \mathscr{H})$, постольку $\sigma_{p} \mathscr{G}=\sigma_{p}\left(\sigma_{p} \mathscr{G} \oplus \sigma_{p} \mathscr{H}\right)$, что значит $\mathrm{ALT} \sigma_{p} \mathscr{G} \geqslant \mathrm{ALT} \sigma_{p} \mathscr{H}$ в силу формулы сложения факторов.

Если $\mathrm{ALT} \sigma_{p} \mathscr{G}>\operatorname{ALT} \sigma_{p} \mathscr{H}$, то $\mathrm{ALT} \sigma_{p} \mathscr{G} \geqslant \operatorname{ALT} \sigma_{p}^{+} \mathscr{H}$ и, тем более, $\operatorname{ALT} \sigma_{p}^{+} \mathscr{G} \geqslant$ $\mathrm{ALT} \sigma_{p}^{+} \mathscr{H}$. Если же $\sigma_{p} \mathscr{G}=\sigma_{p} \mathscr{H}$, то ввиду неравенства ALT $\sigma_{0} \mathscr{G} \geqslant \mathrm{ALT} \sigma_{0} \mathscr{H}$ метарациональность $\sigma_{p} \mathscr{H}$ влечет метарациональность $\sigma_{p} \mathscr{G}$, и потому $\sigma_{p}^{+} \mathscr{G}=\sigma_{p}^{+} \mathscr{H}$. Что и требовалось доказать.

Следуюшие два предложения, по сушеству, являеются формой Второй Теоремы Бокштейна - теоремы о размерностях произведения. 
МУЛЬТИПЛИКАТИВНОСТЬ ФАКТОРОВ БОКШТЕЙНА. Для любыx nолигруnn $\mathscr{G}$, $\mathscr{H}$ и любого простого $p \geqslant 0$ выполнено равенство $\sigma_{p} \mathscr{G} \mathscr{H}=\sigma_{p}\left(\sigma_{p}\left(\mathscr{G} \sigma_{p} \mathscr{H}\right)\right.$.

ФОРМУЛА ФАКТОРА ПРОИЗВЕДЕНИЯ. Пусть $n=\operatorname{alt} \sigma_{p} \mathscr{G}+$ alt $\sigma_{p} \mathscr{H}$, тогда если $\left|\sigma_{p} \mathscr{G}\right| \otimes\left|\sigma_{p} \mathscr{H}\right| \neq 0, \quad$ то $\sigma_{p} \mathscr{G} \mathscr{H}=\left(\left|\sigma_{p} \mathscr{G}\right| \otimes\left|\sigma_{p} \mathscr{H}\right|\right)^{[n]}$, иначе $\sigma_{p} \mathscr{\mathscr { H }}=$ $\left(\left|\sigma_{p} \mathscr{G}\right| *\left|\sigma_{p} \mathscr{H}\right|\right)^{[n-1]}$.

ДокАЗАТЕльство. Докажем сначала формулу $\sigma_{p} \mathscr{G} \mathscr{H}=\sigma_{p}\left(\sigma_{p} \mathscr{G} \sigma_{p} \mathscr{H}\right)$. Не теряя обшности, мы можем предполагать, что $\mathscr{G}=\bigoplus_{p \geqslant 0} \sigma_{p} \mathscr{G}$ и $\mathscr{H}=\bigoplus_{p \geqslant 0} \sigma_{p} \mathscr{H}$. Ввиду взаимной простоты полигрупп $\sigma_{p} \mathscr{G}$ и $\sigma_{q} \mathscr{H}$ при различных $p, q>0$ имеем

$$
\operatorname{ALT} \mathscr{G} \mathscr{H}=\operatorname{ALT}\left(\bigoplus_{p \geqslant 0} \sigma_{p} \mathscr{G} \sigma_{p} \mathscr{H} \oplus \bigoplus_{p>0} \sigma_{0} \mathscr{G} \sigma_{p} \mathscr{H} \oplus \bigoplus_{p>0} \sigma_{p} \mathscr{G} \sigma_{0} \mathscr{H}\right) .
$$

Но второе и третье слагаемые имеют все нетривиальные члены, равные $\mathbb{Q}$, поэтому они не влияют на $p$-факторы правой части при $p>0$. На рациональный фактор правой части они также не влияют, потому что имеют высоты меньшие, чем alt $\sigma_{0} \mathscr{G} \sigma_{0} \mathscr{H}$, что нетрудно получить из рационального неравенства. Поэтому второе и третье слагаемые в сумме справа могут быть опущены. В результате получаем равенство

$$
\operatorname{ALT} \mathscr{G} \mathscr{H}=\operatorname{ALT} \bigoplus_{p \geqslant 0} \sigma_{p} \mathscr{G} \sigma_{p} \mathscr{H}
$$

У суммы справа $p$-фактор, очевидно, равен $\sigma_{p}\left(\sigma_{p} \mathscr{G} \sigma_{p} \mathscr{H}\right)$. Ввиду инвариантности факторов таким же является $p$-фактор произведения $\sigma_{p} \mathscr{G} \mathscr{H}$, что и доказьвает мультипликативность $p$-факторов.

В помош читателю при доказательстве формулы для фактора произведения в случае, когда $\left|\sigma_{p} \mathscr{G}\right| \otimes\left|\sigma_{p} \mathscr{H}\right| \neq 0$, отметим лишь, что тензорное произведение бокштейновских групп всегда само является бокштейновской группой.

Сушествуют лишь два варианта, при которых возникает второй случай. Первьй когда обе высшие группы сомножителей совпадают с $\mathbb{Q}_{p}$, и второй, когда одна из них $\mathbb{Q}_{p}$, а вторая $-\mathbb{Z}_{p}$. Высшая группа произведения во втором случае является суммой трех слагаемых. В первом варианте эти слагаемые таковы: $\mathbb{Q}_{p} * \mathbb{Q}_{p}\left(=\mathbb{Q}_{p}\right), G \otimes \mathbb{Q}_{p}$, $\mathbb{Q}_{p} \otimes G$ (где $G$ - какая-то группа). Последние два либо тривиальны либо факторно эквивалентны $\mathbb{Q}_{p}$, поэтому их сумма изофакторна $\mathbb{Q}_{p}$, что и доказьвает нужную формулу в первом варианте второго случая. Во втором варианте слагаемые таковы: $\mathbb{Q}_{p} * \mathbb{Z}_{p}=\mathbb{Z}_{p}, \mathbb{Q}_{p} \otimes C, C \otimes \mathbb{Z}_{p}$. Второе и третье слагаемые имеют факторную силу, не превосходящую $\mathbb{Z}_{p}$, поэтому их сумма изофакторна $\mathbb{Z}_{p}$. Что и доказьвает формулу во втором варианте.

\section{Коэффициентная высота}

Пусть $C$ какая-то группа, а $\mathscr{G}$ - верхняя полигруппа. Определим высоту alt $_{C} \mathscr{G}$ полигруппы $\mathscr{G}$ относительно группы коэффициентов $C$ как $\operatorname{alt}_{C} \mathscr{G}=\mathscr{G}[\times] C^{(0)}$. Высоту $\operatorname{alt}_{C} \mathscr{G}$ мы будем называть также $C$-коэффициентной высотой или, еще короче, $C$-высотой полигруппы $\mathscr{G}$.

Простые вычисления дают следующий результат. 
ФоРмУЛА КОЭФФИциЕнтной ВЫСоты. $\operatorname{alt}_{C} \mathscr{G}=\operatorname{alt} \mathscr{G} C^{[n]}-n \partial л я n \geqslant \operatorname{alt} \mathscr{G}$.

Следуюшее свойство аддитивности коэффициентных высот непосредственно вытекает из определения.

КОэФФИЦИЕНТнАЯ АДДИТивность. $Е с л и \quad C=\bigoplus_{i \in I} C_{i}, \quad m o \operatorname{alt}_{C} \mathscr{G}=$ $\max _{i} \operatorname{alt}_{C_{i}} \mathscr{G}$.

Следуюшее предложение вскрьвает связь факторной классификации групп с коэффициентной теорией Бокштейна.

КРИТЕРИЙ РАВНОСИЛЬНОСТИ КОЭФФИШИЕНТОВ. Для любъх двух групп $C$ и $D$ следующие два условия эквивалентны:

(1) $C \approx D$;

(2) $\operatorname{alt}_{C} \mathscr{G}=\operatorname{alt}_{D} \mathscr{G}$ для любой верхней полигруппьи $\mathscr{G}$.

ДоКАЗАТЕЛЬСТво. Если $C \approx D$, то согласно критерию равновысотности моногрупш имеем ALT $C^{[n]}=\operatorname{ALT} D^{[n]}$ для любого $n$, откуда alt $\mathscr{G} C^{[n]}-n=\operatorname{alt} \mathscr{G} D^{[n]}-n$, что при $n>\operatorname{alt} \mathscr{G}$ дает $\operatorname{alt}_{C} \mathscr{G}=\operatorname{alt}_{D} \mathscr{G}$.

Если $C$ не изофакторна $D$, то ALT $C^{[2]} \neq \operatorname{ALT} D^{[2]}$ согласно критерию равновысотности для моногрупп. Тогда, в силу леммы о тестировании, найдется бокштейновская моногруппа $\mathscr{B}$ высоты 2 , для которой alt $\mathscr{B} C^{[2]} \neq \operatorname{alt} \mathscr{B} D^{[2]}$, и в силу формулы коэффициентной высоты получаем, что $\operatorname{alt}_{C} \mathscr{B} \neq \operatorname{alt}_{D} \mathscr{B}$, что и требовалось доказать.

Из доказанного критерия и коэффициентной монотонности получаем такое следствие, являюшееся полигрупповой формой Первой Теоремы Бокштейна:

ПоЛнОТА СИСТЕМЫ БОКШТЕЙНА. Для любой группы $C$ и полигруппы $\mathscr{G}$ высота alt $_{C} \mathscr{G}$ равна максимуму высот alt $_{\sigma_{p} C} \mathscr{G}$.

Этот результат вкупе с критерием сравнения для бокштейновских моногрупп дает следуюший результат:

КОЭФФИЦИЕНТНАЯ монотонность. Если группа $C$ факторно доминирует группу $C^{\prime}$, mo $\operatorname{alt}_{C} \mathscr{G} \geqslant \operatorname{alt}_{C^{\prime}} \mathscr{G}$ для любой полигруппь $\mathscr{G}$.

Если $\mathrm{ALT} \mathscr{G} \neq \mathrm{ALT} \mathscr{H}$, то, как мы знаем из леммы о тестировании, найдется бокштейновская моногруппа $\mathscr{B}$ высоты, равной максимуму из высот $\mathscr{G}$ и $\mathscr{H}$, для которой alt $\mathscr{B} \mathscr{G} \neq$ alt $\mathscr{B} \mathscr{H}$. Но из формулы смещения вытекает, что при смещении вверх это неравенство сохранится, и потому получаем такой результат:

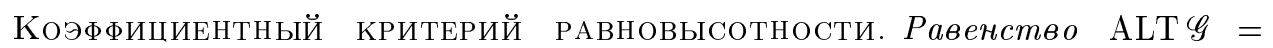
ALT $\mathscr{H}$ равносильно совпадению высот полигрупп $\mathscr{G}$ и $\mathscr{H}$ относительно любой группь коэффициентов.

Формулы умножения позволяют выразить коэффициентную высоту полигрупш относительно групш системы Бокштейна, а значит, и любых груп, через ее моногрупповые факторы Бокштейна. А именно, справедливы следуюшие формулы.

ФОРмУЛЫ КОэФФИцИЕНТнЫХ ВЫСОТ.

(1) $\operatorname{alt}_{\mathbb{Q}} \mathscr{G}=\operatorname{alt} \sigma_{0} \mathscr{G}$

(2) $\operatorname{alt}_{\mathbb{Q}_{p}} \mathscr{G}=\operatorname{alt} \sigma_{p} \mathscr{G}-1$, если $\left|\sigma_{p} \mathscr{G}\right| \neq \mathbb{Z}_{(p)}$, иначе $\operatorname{alt}_{\mathbb{Q}_{p}} \mathscr{G}=\operatorname{alt} \sigma_{p} \mathscr{G} ;$ 
(3) $\operatorname{alt}_{\mathbb{Z}_{p}} \mathscr{G}=\operatorname{alt} \sigma_{p} \mathscr{G}$, если $\left|\sigma_{p} \mathscr{G}\right| \neq \mathbb{Q}_{p}$, иначе alt $_{\mathbb{Z}_{p}} \mathscr{G}=\operatorname{alt} \sigma_{p} \mathscr{G}-1 ;$

(4) $\operatorname{alt}_{\mathbb{Z}_{(p)}} \mathscr{G}=\max \left\{\right.$ alt $\sigma_{0} \mathscr{G}$, alt $\left.\sigma_{p} \mathscr{G}\right\}$

Коэффициентная высота относительно групшы $\mathbb{Z}_{p}$ имеет особое значение, и мы будем ее обозначать через $\mathrm{alt}_{p}$ и назьвать высотой по модулю $p$ или $p$-высотой. Обозначение alt $_{0}$ мы используем для рациональной высоты alt $\mathbb{Q}_{\mathbb{Q}}$. Эти высоты обладают важным свойством.

p-ЛОГАРИФМИЧНОСТЬ УМНОЖЕНИЯ. Для любых полигрупn $\mathscr{G}$ u $\mathscr{H}$ и любого простого $p \geqslant 0$ верно равенство $\operatorname{alt}_{p} \mathscr{G} \mathscr{H}=\operatorname{alt}_{p} \mathscr{G}+\operatorname{alt}_{p} \mathscr{H}$.

ДокАЗАТЕльство. Для $p=0$ результат непосредственно вытекает из мультипликативности нулевого фактора Бокштейна. Если $p>0$, то доказательство получается перебором всех вариантов для типов высших групп $p$-факторов множителей с помошњ формулы для фактора произведения.

Неравенства Бокштейна для полигрупп. Формулы коэффициентных высот позволяют определить коеффициентные высоты полигруппы относительно бокштейновских груп и проверить выполнение неравенств Бокштейна. Другой способ получения этих неравенств - вывод их из групповых неравенств Бокштейна, которые были рассмотрены в конще параграфа о тензорной классификации абелевых групп. Так, очевидно, что неравенство факторных сил групп $B \ll C$ влечет неравенства $\operatorname{alt}_{B} \mathscr{G} \leqslant \operatorname{alt}_{C} \mathscr{G}$ для любой полигруппы $\mathscr{G}$, что для любой полигруппы $\mathscr{G}$ дает неравенства

$$
\operatorname{alt}_{\mathbb{Q}_{p}} \mathscr{G} \leqslant \operatorname{alt}_{\mathbb{Z}_{p}} \mathscr{G} \leqslant \operatorname{alt}_{\mathbb{Z}_{(p)}} \mathscr{G} \geqslant \operatorname{alt}_{\mathbb{Q}} \mathscr{G} \geqslant \operatorname{alt}_{\mathbb{Q}_{p}} \mathscr{G} .
$$

Остальные два неравенства Бокштейна -

$$
\begin{aligned}
\operatorname{alt}_{\mathbb{Q}_{p}} X & \leqslant \max \left\{\operatorname{alt}_{\mathbb{Q}} X, \operatorname{alt}_{\mathbb{Z}_{(p)}} X-1\right\}, \\
\operatorname{alt}_{\mathbb{Z}_{(p)}} X & \leqslant \max \left\{\operatorname{alt}_{\mathbb{Q}} X, \operatorname{alt}_{\mathbb{Q}_{p}} X+1\right\} .
\end{aligned}
$$

Эти неравенства ватекают из групповых неравенств Бокштейна благодаря следуюшему замечанию, доказательство которого мы оставляем читателю.

ЛЕМма О ФУНКЦИЯХ КОФАКТОРНОСТИ. Если группы $C$ и $D$ maковь, что для их функций кофакторности выполнено одно из неравенств $D_{C} \leqslant D_{B}+1$ или $D_{C} \leqslant D_{B}-1$, то аналогичные неравенства выполнены для относительных выссот: $\operatorname{alt}_{C} \leqslant \operatorname{alt}_{B}+1 u$ alt $_{C} \leqslant \operatorname{alt}_{B}-1$.

Заметим, что именно ради справедливости этого утверждения индекс кофакторности определен как $\max \{3 \operatorname{triv}(G \otimes H), 2 \operatorname{triv}(G * H)\}$, а не как $\max \{2 \operatorname{triv}(G \otimes H)$, $\operatorname{triv}(G * H)\}$.

\section{Нижняя алгебра Кюннета}

Полигруппу $\mathscr{G}$ мы называем нижней, если $G_{0}=0$. Нижние полигрупшы отличаются от верхних начальным условием и отсутствием требования конечности высоты. Полигруп, нижних и верхних одновременно, не существует. В этом разделе полигрупшы по умолчанию нижние. 
Сумма нижних полигрупп определяется почленно так же, как и для верхних. Произведение их определяется так:

$$
\mathscr{G}\langle\times\rangle \mathscr{H}=\left\{\bigoplus_{i+j=n}\left(G_{i} \otimes H_{j}\right) \oplus \bigoplus_{i+j=n-1}\left(G_{i} * H_{j}\right)\right\}_{n \geqslant 0},
$$

и называется нижним произведением Кюннета полигрупп. Мы, однако, как и в случае верхних полигрупп, будем обычно опускать знак произведения.

Глубиной нижней полигруппы $\mathscr{G}$ назьвается размерность наименьшего нетривиального члена этой полигрупп; обозначение: $\operatorname{dep} \mathscr{G}$. Таким образом, $\operatorname{dep} \mathscr{G}>0$ для любой нижней полигрупшы, а равенство $\operatorname{dep} \mathscr{G}=\infty$ характеризует нулевую полигруппу, т.е. полигрупу, все члены которой тривиальны. Для нижнего произведения и суммы очевидны следуюшие соотношения:

$$
\operatorname{dep} \mathscr{G} \mathscr{H} \geqslant \operatorname{dep} \mathscr{G}+\operatorname{dep} \mathscr{H}, \quad \operatorname{dep}(\mathscr{G} \oplus \mathscr{H})=\min \{\operatorname{dep} \mathscr{G}, \operatorname{dep} \mathscr{H}\} .
$$

Нижние полигрупшы $\mathscr{G}$ и $\mathscr{H}$ назьваются взаимно простыли, если $\operatorname{dep} \mathscr{G} \mathscr{H}=\infty$, что означает взаимную простоту всех их членов.

Очевидно, что сумма и нижнее произведение нижних полигрупп является нижней полигруппой. Подобно тому как верхнее произведение определяет высотность для верхних полигруп, нижнее произведение определяет глубинный тип, или глубинность, для нижних полигрупп.

Теория глубинных типов строится аналогично теории высотных типов. Через $\mathfrak{K}_{*}$ обозначается нижняя алгебра Кюннета - решетка глубинных типов с операциями сложения и умножения. Нулевая полигруппа является наибольшим элементом этой алгебры, а наименшшим является тип, представленный полигрупой $\mathbb{Z}^{(1)}$.

Для нижней полигруппы $\mathscr{G}$ через $|\mathscr{G}|$ обозначается ее низшая (т.е. наименьшей размерности) нетривиальная группа, а через $\lfloor\mathscr{G}\rfloor$ - низшая моногрупша, определяемая как $|G|^{(\operatorname{dep} G)}$.

Для тривиальной полигрупш $\mathscr{G}$ считаем $|\mathscr{G}|=0$. Нижняя полигруппа назьвается моногруппой, если у нее есть только одна нетривиальная группа. Для групшы $G$ полигруппа $G^{(n)}$ является нижней моногруппой глубины $n$, для которой $\left|G^{(n)}\right|=G$.

Следуюшие результаты для нижних полигруп доказьваются аналогично соответствуюшим результатам для верхних.

КРИТЕРИЙ РАВНОГЛУБИННОСТИ МОНОГРУПП. Для нижних моногрупп равенство $\mathrm{DEP} \mathscr{G}=\mathrm{DEP} \mathscr{H}$ равносильно равенству $|\mathscr{G}| \approx|\mathscr{H}|$.

ИЗОФАКТОРНАЯ ОПРЕДЕЛЕННОСТЬ ГЛУБИННОСТИ. $\mathscr{G} \approx \mathscr{H}$ влечет $\mathrm{DEP} \mathscr{G}=$ DEP $\mathscr{H}$ для любых нижних полигрупп.

НИЖняя фоРмУЛА СмЕШЕНИя. Если $\mathscr{B}$ и $\mathscr{B}^{\prime}-$ моногруппы и $|\mathscr{B}|=\left|\mathscr{B}^{\prime}\right|$, то

$$
\operatorname{dep} \mathscr{B}^{\prime} \mathscr{G}=\operatorname{dep} \mathscr{B} \mathscr{G}+\operatorname{dep} \mathscr{B}^{\prime}-\operatorname{dep} \mathscr{B} .
$$

Нижняя формула смещения проще верхней. Это приводит к упрощению доказательства леммы о тестировании. Результат же остается без изменений. Бокштейновский полигруповой фактор $\Sigma_{p} \mathscr{G}$ для нижней полигрупшы $\mathscr{G}$ определяется так же, 
как для верхней. Бокштейновский моногрупповой фактор $\sigma_{p} \mathscr{G}$ для нижней полигруппы определяется как $\left\lfloor\Sigma_{p} \mathscr{G}\right\rfloor$. Для нижних полигруп, в отличие от верхних, $p$-фактор Бокштейна может оказаться тривиальным.

При этом формулы умножения на нижние бокштейновские моногрупшы останутся такими же, как и для верхних, за исключением четвертой, где максимум заменится на минимум. Доказательства при этом не меняются.

НИЖНИЕ фОРМУЛЫ УМНОЖЕНИЯ. Для любой нижней полигруппы $\mathscr{G}$ справедливы следуюшие формуль:

(1) $\operatorname{dep} \mathscr{G} \mathbb{Q}^{(n)}=\operatorname{dep} \sigma_{0} \mathscr{G}+n$;

(2) $\operatorname{dep} \mathscr{G} \mathbb{Q}_{p}^{(n)}=\operatorname{dep} \sigma_{p} \mathscr{G}+n+\left(\mathbb{Q}_{p},\left|\sigma_{p} \mathscr{G}\right|\right)-3 ;$

(3) $\operatorname{dep} \mathscr{G} \mathbb{Z}_{p}^{(n)}=\operatorname{dep} \sigma_{p} \mathscr{G}+n+\left(\mathbb{Z}_{p},\left|\sigma_{p} \mathscr{G}\right|\right)-3$;

(4) $\operatorname{dep} \mathscr{G} \mathbb{Z}_{(p)}^{(n)}=\min \left\{\operatorname{dep} \sigma_{0} \mathscr{G}+n, \operatorname{dep} \sigma_{p} \mathscr{G}+n\right\}$.

МЕТАРАЦИОНАЛЬНАЯ АЛЬТЕРНАТИВА. Для любого $k \geqslant 0$ справедливо равенcmво $\operatorname{DEP}\left(\mathbb{Q}^{(n)} \oplus \mathbb{Z}_{(p)}^{[n+k+1]}\right)=\operatorname{DEP}\left(\mathbb{Q}^{(n)} \oplus \mathbb{Q}_{p}^{(n+k)}\right)$.

Из-за метарациональной альтернативы бокштейновские факторы не являются инвариантами глубинного типа и их также приходится унифищировать.

Нижний унифииированный моногрупповой $p$-фактор $\sigma_{p}^{-} \mathscr{G}$ определяется как $\sigma_{p} \mathscr{G}$ в случае, если $\left|\sigma_{p} \mathscr{G}\right| \neq \mathbb{Z}_{(p)}$ или $\operatorname{dep} \sigma_{p} \mathscr{G} \leqslant \operatorname{dep} \sigma_{0} \mathscr{G}$, и как $\mathbb{Q}_{p}^{\left(\operatorname{dep} \sigma_{p} \mathscr{G}-1\right)}$ в противном случае. Нижние унифицированные факторы Бокштейна оказьваются полной системой инвариантов глубинного типа.

Разложение Бокштейна в нижней алгебре выглядит так же, как в верхней:

$$
\operatorname{DEP} \bigoplus_{p \geqslant 0} \sigma_{p} \mathscr{G}=\operatorname{DEP} \bigoplus_{p \geqslant 0} \sigma_{p}^{-} \mathscr{G}=\operatorname{DEP} \mathscr{G} .
$$

Факторы Бокштейна для нижних полигрупп, как и для верхних имеют свойства аддитивности и мультипликативности:

$$
\sigma_{p}(\mathscr{G} \oplus \mathscr{H})=\sigma_{p}\left(\sigma_{p} \mathscr{G} \oplus \sigma_{p} \mathscr{H}\right), \quad \sigma_{p} \mathscr{G} \mathscr{H}=\sigma_{p}\left(\sigma_{p} \mathscr{G} \sigma_{p} \mathscr{H}\right) .
$$

ФОРМУЛА ФАКТОРА ПРОИЗВЕДЕНИЯ. Пусть $n=\operatorname{dep} \sigma_{p} \mathscr{G}+\operatorname{dep} \sigma_{p} \mathscr{H}$, тогда если $\left|\sigma_{p} \mathscr{G}\right| \otimes\left|\sigma_{p} \mathscr{H}\right| \neq 0$, mо $\sigma_{p} \mathscr{G} \mathscr{H}=\left(\left|\sigma_{p} \mathscr{G}\right| \otimes\left|\sigma_{p} \mathscr{H}\right|\right)^{(n)}$, иначе $\sigma_{p} \mathscr{G} \mathscr{H}=\left(\left|\sigma_{p} \mathscr{G}\right| *\right.$ $\left.\left|\sigma_{p} \mathscr{H}\right|\right)^{(n+1)}$.

Глубина полигрушы $\mathscr{G}$ относительно групы коэффищиентов $C$ определяется как $\operatorname{dep}_{C} \mathscr{G}=\mathscr{G}\langle\times\rangle C^{(0)}$. И, очевидно, $\operatorname{dep}_{C}=\left\{\operatorname{dep} \mathscr{G} C^{(n)}-n\right\}$ для любого $n$. Коэффициентная аддитивность, монотонность, коэффициентный критерий равноглубинности, критерий равносильности коэффициентов и теорема о базисности системы Бокштейна для нижних полигрупп формулируются и доказываются так же, как для верхних.

ФоРмУлЫ КОЭФФИЦИЕНТНЫХ ГЛУБИн.

(1) $\operatorname{dep}_{\mathbb{Q}} \mathscr{G}=\operatorname{dep} \sigma_{0} \mathscr{G}$

(2) $\operatorname{dep}_{\mathbb{Q}_{p}} \mathscr{G}=\operatorname{dep} \sigma_{p} \mathscr{G}+1$, если $\left|\sigma_{p} \mathscr{G}\right| \neq \mathbb{Z}_{(p)}$, иначе $\operatorname{dep}_{\mathbb{Q}_{p}} \mathscr{G}=\operatorname{dep} \sigma_{p} \mathscr{G} ;$

(3) $\operatorname{dep}_{\mathbb{Z}_{p}} \mathscr{G}=\operatorname{dep} \sigma_{p} \mathscr{G}$, если $\left|\sigma_{p} \mathscr{Y}\right| \neq \mathbb{Q}_{p}$, иначе $\operatorname{dep}_{\mathbb{Z}_{p}} \mathscr{G}=\operatorname{dep} \sigma_{p} \mathscr{G}+1$;

(4) $\operatorname{dep}_{\mathbb{Z}_{(p)}} \mathscr{G}=\min \left\{\operatorname{dep} \sigma_{0} \mathscr{G}, \operatorname{dep} \sigma_{p} \mathscr{G}\right\}$. 
Коэффициентная глубина относительно $\mathbb{Z}_{p}$ назьвается $p$-глубиной или глубиной по модулю $p$ и обозначается $\operatorname{dep}_{p}$. Через $\operatorname{dep}_{0}$ обозначается рациональная глубина. И так же, как в верхнем случае, доказывается для любого простого $p \geqslant 0$ логарифмический закон

$$
\operatorname{dep}_{p} \mathscr{G} \mathscr{H}=\operatorname{dep}_{p} \mathscr{G}+\operatorname{dep}_{p} \mathscr{H}
$$

\section{Отношение совместимости}

Будем говорить, что пара $\mathscr{G}, \mathscr{H}$, состояшая из верхней полигруппы $\mathscr{G}$ и нижней полигруппы $\mathscr{H}$, совместима, и писать $\mathscr{G} \tau \mathscr{H}$, если $G_{n} \otimes H_{m}=0$ при $n>m$ и $G_{n} * H_{m}=0$ при $n>m+1$. Как нетрудно видеть, условие совместимости можно выразить эквивалентным образом следующими двумя способами

ВЫСОТНЫЙ КРИТЕРИЙ СОВМЕСТИМОстИ. Следующие три условия равносильньц для пары $\mathscr{G}, \mathscr{H}$ из верхней и нижней полигрупп:

(1) $\mathscr{G} \tau \mathscr{H}$

(2) alt $\mathscr{G} H_{n}^{[n+1]} \leqslant 2 n+1$ для любого $n>0$;

(3) $\operatorname{alt}_{H_{n}} \mathscr{G} \leqslant n$ для любого $n>0$.

ГЛУБИННЫЙ КРИТЕРИЙ СОВМЕСТИМОсТИ. Следующие три условия равносильньг для пары $\mathscr{G}, \mathscr{H}$ из верхней и нижней полигрупп:

(1) $\mathscr{G} \tau \mathscr{H}$;

(2) $\operatorname{dep} G_{n}^{(n-1)} \mathscr{H} \geqslant 2 n-1$ для любого $n>0$;

(3) $\operatorname{dep}_{G_{n}} \mathscr{H} \geqslant n$ для любого $n>0$.

Из высотного критерия вытекает, что при замене $\mathscr{G}$ на равновысотную полигруппу $\mathscr{G}^{\prime}$ условие совместимости сохраняется. Из глубинного критерия вытекает, что при замене полигрупш $\mathscr{H}$ на равноглубинную условие совместимости также сохраняется. Таким образом, получаем следуюшее утверждение.

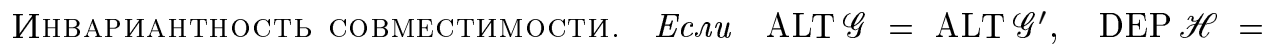
$\mathrm{DEP} \mathscr{H}^{\prime} u \mathscr{G} \tau \mathscr{H}$, mo $\mathscr{G}^{\prime} \tau \mathscr{H}^{\prime}$.

Таким образом, отношение совместимости является, по сушеству, отношением между высотньми и глубинными типами полигрупп.

Если $\mathscr{G} \tau \mathscr{H}$ и $\operatorname{ALT}^{\mathscr{G}} \mathscr{G}^{\prime} \leqslant \mathrm{ALT} \mathscr{G}$, то для любого $n$ имеем неравенства alt $\mathscr{G} H_{n}^{[n]} \leqslant n$ и alt $\mathscr{G}^{\prime} H_{n}^{[n]} \leqslant \operatorname{alt} \mathscr{G} H_{n}^{[n]}$, откуда alt $\mathscr{G}^{\prime} H_{n}^{[n]} \leqslant n$, и, следовательно, $\mathscr{G}^{\prime} \tau \mathscr{H}$. Аналогично доказьвается, что отношение совместимости сохраняется при увеличении типа $\mathscr{H}$. Таким образом, справедливо следующее предложение.

Монотонность Совместимости. Если $\mathscr{G} \tau \mathscr{H} \quad u \quad \operatorname{ALT} \mathscr{G}^{\prime} \leqslant \operatorname{ALT} \mathscr{G}, a$ $\mathrm{DEP} \mathscr{H}^{\prime} \geqslant \mathrm{DEP} \mathscr{H}$, mo $\mathscr{G}^{\prime} \tau \mathscr{H}^{\prime}$.

Отметим такие свойства аддитивности отношения совместимости:

Нижняя АдДитивность. Пусть $\mathscr{G} \tau \mathscr{H}_{i}$ для любой нижней полигруппь из некоторого индексированного $i \in I$ семейства, тогда $\mathscr{G} \tau \bigoplus_{i \in I} \mathscr{H}_{i}$. 
ВЕРХняя АДДИТИВность. Пусть $\mathscr{G}_{i} \tau \mathscr{H}$ для любой верхней полигруппь из некоторого индексированного $i \in I$ семейства, тогда $\bigoplus_{i \in I} \mathscr{G}_{i} \tau \mathscr{H}$.

ДокАЗАТЕЛЬСтвО. Ограничимся доказательством верхней аддитивности. Воспользуемся высотным критерием совместимости. При любом $i \in I$ и любом натуральном $n$ имеем alt $\mathscr{G}_{i} H_{n}^{[n]} \leqslant n$. A поскольку alt $\bigoplus_{i \in I} \mathscr{G}_{i} H_{n}^{[n]}=\max$ alt $\mathscr{G}_{i} H_{n}^{[n]}$, постольку $\bigoplus_{i \in I} \mathscr{G}_{i} H_{n}^{[n]} \leqslant n$, и согласно тому же критерию получаем $\bigoplus_{i \in I} \mathscr{G}_{i} \tau \mathscr{H}$.

ФАКТОРНЫЙ КРИТЕРИЙ СОВМЕСТИМОСТИ. Полигруппь $\mathscr{G}$ и $\mathscr{H}$ совместимь $\mathscr{G} \tau \mathscr{H}$, если и только если при любом простом $p \geqslant 0$ совместимы их унифицированные факторы Бокштейна $\sigma_{p}^{+} \mathscr{G} \tau \sigma_{p}^{-} \mathscr{H}$.

ДокАЗАТЕЛЬСтво. Если $\mathscr{G} \tau \mathscr{H}$, то неравенства ALT $\sigma_{p}^{+} \mathscr{G} \leqslant \mathrm{ALT} \mathscr{G}$ и DEP $\mathscr{H} \leqslant$ $\mathrm{DEP} \sigma_{p}^{-} \mathscr{H}$ влекут за собой отношение $\sigma_{p}^{+} \mathscr{G} \tau \sigma_{p}^{-} \mathscr{H}$ в силу монотонности отношения совместимости. Доказательство обратного утверждения, ввиду разложений ALT $\mathscr{G}=$ $\mathrm{ALT} \bigoplus \sigma_{p}^{+} \mathscr{G}$ и $\mathrm{DEP} \mathscr{H}=\mathrm{DEP} \bigoplus \sigma_{p}^{-} \mathscr{H}$, сводится благодаря свойствам верхней и нижней аддитивности отношения совместимости к проверке вьполнения соотношений $\sigma_{p}^{+} \mathscr{G} \tau \sigma_{q}^{-} \mathscr{H}$ при всевозможных парах $p, q$. При этом нам известно, что при $p=q$ соотношения вьполняются. Но при $p \neq q$ любой $p$-фактор взаимно прост с $q$-фактором, поэтому отношение $\sigma_{p}^{+} \mathscr{G} \tau \sigma_{q}^{-} \mathscr{H}$ вьполняется для любых полигрупп $\mathscr{G}$ и $\mathscr{H}$.

Верхняя полигруппа $\mathscr{G}$ и нижняя полигруппа $\mathscr{H}$ называются $n$-двойственнылми, если $H_{k}=G_{n-k-1}$ при $k<n$ и $G_{k}=H_{k}=0$ при $k \geqslant n$.

КРИТЕРИЙ ДВОЙСТВЕНнОСТИ. Пусть верхняя полигруппа $\mathscr{G}$ и нижняя $\mathscr{H}$ являются $n$-двойственными, тогда для любой верхней полигруппь $\mathscr{F}$ неравенство alt $\mathscr{F} \mathscr{G}<n$ равносильно отношению $\mathscr{F} \tau \mathscr{H}$.

ДокАЗАТЕЛЬСТвО. Первое условие для тензорных произведений расшифровывается так: $F_{i} \otimes G_{j}=0$ при $i+j>n-1$. Пусть $k=n-j-1$, тогда это неравенство можно преобразовать в следующее: $i>k$, что как раз и является тензорньм условием отношения $\mathscr{F} \tau \mathscr{H}$ ввиду того, что $H_{k}=G_{j}$. Аналогично проверяется равносильность периодических условий.

\section{$\S$ 3. Кодовая решетка}

\section{Биаддитивные решетки}

Биаддитивной решеткой называется множество $L$ с заданным на нем отношением частичного порядка $\leqslant$ и парой аддитивно записьваемых операций, обозначаемых $\langle+\rangle$ и [†] и назьваемых соответственно мажорным.м и минорным сложением, если для любых $x, y, z \in L$ вьполнены следующие условия.

(1) Сушествуют $\sup \{x, y\}$ и $\inf \{x, y\}$.

(2) Операции $[+]$ и $\langle+\rangle$ коммутативны и ассоциативны.

(3) Обе операции имеют общий нейтральный элемент.

(4) $x\langle+\rangle \inf \{y, z\}=\inf \{x y\}\langle+\rangle \inf \{x, z\}, x[+] \sup \{y, z\}=\sup \{x y\}[+] \sup \{x, z\}$.

(5) $x\langle+\rangle(y[+] z) \geqslant(x\langle+\rangle y)[+] z$. 
Первое условие означает решеточную упорядоченность $L$. Второе означает, что $L$ является абелевой полугруппой по отношению к обеим операциям. Общий нейтральный элемент операций обозначаем как 0 и назьваем аддитивнылм нулем решетки. Четвертое условие выражает согласованность сложений с порядком и называется парной дистрибутивностью сложений по отношению к порядку. Последнее условие называется парной ассоциативностью. При подстановке $y=0$ из неравенства парной ассоциативности получается неравенство между суммами $x\langle+\rangle z \geqslant x[+] z$, оправдываюшее их названия.

Биаддитивная решетка называется полной, если каждое подмножество в ней имеет точную верхнюю и нижнюю грань и, кроме того, вьполнено следующее усиленное свойство парной дистрибутивности сложений по отношению к порядку:

$$
x\langle+\rangle \inf A=\inf \{x\langle+\rangle a \mid a \in A\} \quad x[+] \sup A=\sup \{x[+] a \mid a \in A\} .
$$

В полной решетке есть наибольший и наименьший элементы. В дальнейшем мы будем иметь дело, в основном, с полными решетками, и потому по умолчанию считаем решетки полными.

Бесконечный элемент. Элемент решетки мы будем назьвать бесконечныцм относительно какой-то операции сложения, если его сумма относительно этой операции с любым элементом решетки равна ему самому.

Ясно, что бесконечный элемент для каждой операции один. Если бесконечный элемент положителен (т.е. больше нуля), то он совпадает с наибольшим элементом решетки. Действительно, пусть $i-$ бесконечньй элемент относительно операции,+ a $M-$ наибольший элемент решетки. Тогда $i=i+M \geqslant 0+M=M$. Аналогично, отрицательньй бесконечный элемент обязательно является наименьшим элементом решетки.

Сигнатурная триада. Простейшим примером биаддитивной решетки является сигнатурная триада. Она состоит всего из трех элементов $\mathfrak{S}=\{-, 0,+\}$, записанных в порядке возрастания и называемых соответственно минусом или отрицательной сигнатурой, нулем и плюсом или положительной сигнатурой. Операции на сигнатурной триаде определяются согласно следуюшим правилам:

(1) $x\langle+\rangle x=x[+] x=x$ (идемпотентность);

(2) $x\langle+\rangle=x[+] 0=x$ (нейтральность нуля);

(3) $-\langle+\rangle+=+$ и $-[+]+=-$.

Плюс является мажсорно-бесконечным элементом сигнатурной триады, а минус минорно-бесконечным. Проверим парную ассоциативность сигнатур. Если $x=-$, то левая часть отрищательна, и потому неравенство вьполнено при любой правой части. Если $z=+$, то правая часть положительна, что и обеспечивает справедливость неравенства.

Пусть теперь $x \geqslant 0 \geqslant z$. Тогда если $y=-$, то $y\langle+\rangle z=-$, и левая часть отрицательна и неравенство справедливо. Если $y=+$, то аналогичным образом получаем, что правая часть положительна и неравенство вьполнено. Наконец, если $\operatorname{sgn} y=0$, то выражение слева равняется $x[+] z$, так же как и выражение справа.

Двухскобочные знаки. В дальнейшем, чтобы не дублировать одни и те же формулы, мы будем использовать двухскобочные знаки: $[\langle+\rangle],\langle[+]\rangle,[\langle \pm\rangle],[\langle\mp],\langle[ \pm]\rangle,\langle\{\mp]\rangle$. 
Всякое арифметическое выражение, содержашее двухскобочные знаки, представляет собой запись двух выражений. Одно получается, если во всех двухскобочных знаках оставить внешнюю скобку, убрав внутреннюю, а другое - если убрать внешнюю, оставив внутреннюю. При этом мы считаем, что $\langle \pm\rangle=[\mp]=+$ и $[ \pm]=\langle\mp\rangle=-$.

Так, правила сложения сигнатур могут быть выражены следуюшими тремя формулами: $x[\langle+\rangle] x=x, x[\langle+\rangle] 0=x,+[\langle+\rangle]-=[\langle \pm\rangle]$. А формула $\pm[\langle+\rangle] 0= \pm$ представляет собой сокрашенную запись сразу четырех выражений, которые получаются, когда мы независимо расшифровываем двойные и двухскобочные знаки.

Разности. На всякой полной решетке $L$ можно ввести операции мажсрной, обозначаемой $\langle-\rangle$, и минорной, обозначаемой [-], разностей следующим образом:

$$
\alpha[-] \beta=\sup \{\gamma \mid \gamma[+] \beta \leqslant \alpha\}, \quad \alpha\langle-\rangle \beta=\inf \{\gamma \mid \gamma\langle+\rangle \beta \geqslant \alpha\} .
$$

Если $\beta\langle+\rangle \gamma \ngtr \alpha$ при любом $\gamma \in L$, мы считаем мажорную разность $\alpha\langle-\rangle \beta$ неопределенной. Аналогично, если $\beta[+] \gamma \not \alpha$ при любом $\gamma \in L$, то мы считаем неопределенной минорную разность $\alpha[-] \beta$.

Если решетка содержит положительный бесконечный элемент $+\infty$, то мажорная разность сушествует для любых операндов, а если содержит $-\infty$, то минорная разность всегда определена.

Следуюшая таблица содержит все результаты, касаюшиеся вычисления минорной и мажорной разностей в сигнатурной триаде. Уменьшаемое берется из левого столбца таблицы, а вычитаемое - из верхней строки. Разность находится на пересечении строки уменьшаемого со столбцом вычитаемого. Результат минорного вычитания получается при выборе квадратных скобок в соответствуюшем двухскобочном выражении, а результат мажорного - при выборе круглых.

Таблица вьчитания сигнатур

\begin{tabular}{|c|c|c|c|}
\hline$\langle[-]\rangle$ & + & 0 & - \\
\hline+ & $\langle[\mp]\rangle$ & + & + \\
\hline 0 & - & 0 & + \\
\hline- & - & - & $\langle[\mp]\rangle$ \\
\hline
\end{tabular}

Пополненный натуральный ряд. Множество, получающееся при добавлении к натуральному ряду нуля и $+\infty$, называется пополненным натуральным рядом и обозначается $\widetilde{\mathbb{N}}$. Ноль является наименьшим элементом $\widetilde{\mathbb{N}}, \mathrm{a}+\infty-$ наибольшим. Порядок на конечных натуральных числах обьчньй. На пополненном натуральном ряде определяется операция сложения так, что для конечных слагаемых она совпадает с обычной арифметической. A $+\infty$ является бесконечным элементом этой операции. Минорная и мажорная разности натуральных чисел совпадают с арифметической, если уменьшаемое не меньше чем вычитаемое, иначе мажорная разность равна нулю, а минорная не существует. 


\section{Основные неравенства}

Непосредственно из определения разностей вытекают такие их свойства:

ПРИНЦИПЫ ПЕРЕНОСА. $x[+] y \leqslant z \Longleftrightarrow x \leqslant z[-] y, x\langle+\rangle y \geqslant z \Longleftrightarrow x \geqslant z\langle-\rangle y$.

Если $x \geqslant y$, то $\sup \{x, y\}=x$ и $\inf \{x, y\}=y$. Поэтому условие парной дистрибутивности позволяют получить такой результат.

КовАРИАНТНОСТь СЛОЖЕНИЯ. $x \leqslant y$ влечет $x\langle+\rangle z \leqslant y\langle+\rangle z u x[+] z \leqslant y[+] z$.

Ясно, что любая разность не убывает при увеличении уменьшаемого (ковариантна уменшшаемому) и не возрастает при увеличении вычитаемого (контравариантна вычитаемому).

ВАРИАНТНОСТЬ ВЫЧИТАНИЯ. $z \geqslant x$ влечет $z\langle[-]\rangle y \geqslant x\langle[-]\rangle y u y\langle[-]\rangle x \geqslant y\langle[-]\rangle z$.

Следующие неравенства будут часто применяться далее.

КОМПЕНСАЦИОННЫЕ НЕРАВЕНСТВА. Для любых әлементов биаддитивной решетки выполняются следующие неравенства:

$$
(x\langle-\rangle y)\langle+\rangle y \geqslant x \geqslant(x[-] y)[+] y, \quad(x\langle+\rangle y)\langle-\rangle y \leqslant x \leqslant(x\langle+\rangle y)[-] y
$$

ДокАЗАТЕЛЬСТВО. Если для любого $z$ из некоторого множества $Z$ выполнено неравенство $z\langle+\rangle y \geqslant x$, то в силу дистрибутивности будет $\inf Z\langle+\rangle y \geqslant x$. Это доказывает неравенство $(x\langle-\rangle y)\langle+\rangle y \geqslant x$. Аналогично доказывается неравенство $(x[-] y)\langle+\rangle y \leqslant x$. Если в неравенстве $x\langle+\rangle y \leqslant x\langle+\rangle y$ перенести согласно правилам переноса $y$ справа налево, то получится неравенство $(x\langle+\rangle y)\langle-\rangle y \leqslant x$, а если в неравенстве $x[+] y \leqslant x[+] y$ перенести $y$ направо, то получится $x \leqslant(x[+] y)[-] y$.

ТРАНСПОЗИЦИОННЫЕ НЕРАВЕНСТВА. При любы $x, y, z$ справедливы неравенства

$$
(x[\langle+\rangle] z)\langle-\rangle y \leqslant(x\langle-\rangle y)[\langle+\rangle] z, \quad(x[\langle+\rangle] z)[-] y \geqslant(x[-] y)[\langle+\rangle] z .
$$

ДокаЗАТЕльство. Докажем первое неравенство. В силу общих принципов переноса оно равносильно следующему: $((x\langle-\rangle y)[\langle+\rangle z)\langle+\rangle y \geqslant x[\langle+\rangle z$. А последнее доказывается цепочкой неравенств, используя парную ассоциативность и компенсационные неравенства $((x\langle-\rangle y)[\langle+\rangle])\langle+\rangle y \geqslant((x\langle-\rangle y)\langle+\rangle y)[\langle+\rangle z \geqslant x[\langle+\rangle] z$. Второе неравенство доказьвается аналогично.

Двухсторонние операции и регулярные элементы. В случае, когда минорная сумма совпадает с мажорной: $x[+] y=x\langle+\rangle y$, мы будем говорить, что определена двухсторонняя сумма. И будем в этом случае опускать скобочки и записывать эту сумму как $x+y$. Аналогичное соглашение мы применяем к разности, используя запись $x-y$ в случае, если совпадают односторонии разности $x[-] y=x\langle-\rangle y$, и назьвая такую разность односторонней. Элемент $n$ называется регулярнылм, если справедливость любого из неравенств $x\langle[+]\rangle n \leqslant y\langle[+]\rangle n$ влечет неравенство $x \leqslant y$. Непосредственно из определения регулярного элемента вытекает такой 
ПРИНЦИП СОКРАШЕНИЯ. Если $n$ регулярный, то при любых $x, y$ имеем:

$$
(x[\langle+\rangle n)\langle-\rangle(y[\langle+\rangle n)=x\langle-\rangle y, \quad(x[\langle+\rangle] n)[-](y[\langle+\rangle] n)=x[-] y .
$$

Односторонние порядки. Две операции разности индуцируют на аддитивной решетке в дополнение к основному отношению порядка $\leqslant$ еше два: мажорныц $\langle\leqslant\rangle$ и минорный $[\leqslant]$. Отношение $x[\geqslant\rangle] y$ расшифровывается так: $x=y[\langle-\rangle] z$ для некоторого $z \geqslant 0$. Транзитивность отношений [ $\geqslant]$ вытекает из следуюшего предложения.

ФормУлы ПОВТОРНОГО ВЫЧИТАНИя. $(x\langle[-]\rangle y)\langle[-]\rangle z=x\langle[-]\rangle(y\langle[+]\rangle z)$.

ДокАЗАТЕЛЬство. Левая часть мажорной формулы определяется как нижняя грань множества $A=\{w \mid w\langle+\rangle z \geqslant x\langle-\rangle y\}$. Перенося $y$ влево и группируя $z$ и $y$, получаем $A=\{w \mid w\langle+\rangle(z\langle+\rangle y) \geqslant x\}$, но это уже множество, нижняя грань которого определяет $x\langle-\rangle(y+z)$. Таким образом, мажорное равенство доказано. Минорное доказывается аналогично.

Отношение $x\langle\leqslant\rangle$ мы будем выражать словами, говоря, что $x$ мажорно подчинен $y$. Аналогично, $x$ минорно подчинен $y$, если $x[\leqslant] y$.

УРАВНЕНИЕ ПОДЧИНЕНИЯ. $x[\geqslant] y \Longleftrightarrow x=y[\langle-\rangle](y[\langle-\rangle x)$.

ДоКАЗАТЕльство. Если $x=y[\langle-\rangle(y[\langle-\rangle x)$, то, полагая $z=y[\langle-\rangle]$, получаем

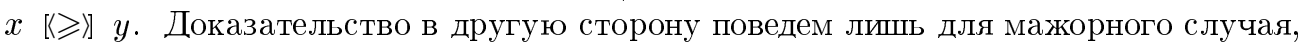
оставив минорный читателю. Если $x\langle\leqslant\rangle y$, то $x=y\langle-\rangle z$ для некоторого $z$. Тогда $z+x \geqslant y$, откуда $z \geqslant y\langle-\rangle x$. И в силу контравариантности разности вычитаемому отсюда следует неравенство $x=y\langle-\rangle z \leqslant y\langle-\rangle(y\langle-\rangle x)$. Но обратное неравенство $x \geqslant y\langle-\rangle(y\langle-\rangle x)$ также верно, ибо равносильно верному неравенству $x+(y\langle-\rangle x) \geqslant y$. Что и требовалось доказать.

Будем говорить, что сумма $x\langle+\rangle y$ представляет собой минимальное разложение элемента $z$, и писать $z\langle=\rangle x\langle+\rangle y$, если $z \leqslant x\langle+\rangle y$ и для любых $x^{\prime}<x$ и $y^{\prime}<y$ будет $x^{\prime}\langle+\rangle y \ngtr z$ и $x\langle+\rangle y^{\prime} \ngtr z$. Непосредственно из определений мажорной разности вытекает

КРИТЕРИЙ МИНИМАЛЬНОСТИ. $z\langle=\rangle x+y \Longleftrightarrow x=z\langle-\rangle$ y $u=z\langle-\rangle x$.

Из этого критерия получаем

СЛЕДСТВИЕ. Если $z\langle=\rangle x+y$, mo $x\langle\leqslant\rangle z u y\langle\leqslant\rangle z$.

Формулировку и доказательство аналогичных результатов про максимальные разложсения мы оставляем читателю.

Опираясь на таблицу вычитания сигнатур, нетрудно получить таблицы сравнения для односторонних порядков.

$$
-\langle<\rangle+\langle<\rangle 0, \quad+[<]-[<]<0
$$

\section{Триплеты}

Множество триплетов, обозначаемое $\mathbb{N}^{ \pm}$, является подмножеством прямого произведения $\widetilde{\mathbb{N}} \times \mathfrak{S}$, содержашим все элементы этого произведения, за исключением следуюших пяти: $(+\infty,+),(+\infty,-),(1,-),(0,+),(0,-)$, называемых некорректныли триплетами. 
Для триплета $\alpha=(a, b)$ его первая координата $a$ называется основой, а вторая координата $b$ - сигнатурой. Основа триплета $\alpha$ обозначается $|\alpha|$, а сигнатура $\operatorname{sgn} \alpha$. Для триплетов определяются две односторонние операции сложения: мажорная и минорная, обозначаемые $\langle+\rangle$ и [+] соответственно. Формулы, задающие эти операции для конечных триплетов, таковы:

$$
\mid x[\langle+\rangle y|=| x|+| y \mid, \quad \operatorname{sgn} x[\langle+\rangle]=\operatorname{sgn} x[\langle+\rangle] \operatorname{sgn} y .
$$

$\mathrm{A}+\infty$ является бесконечньм элементом относительно обеих операций, т.е. $+\infty[\langle+\rangle] x$ $=+\infty$ для любого триплета $x$. Триплеты с конечной основой $(n,+)$ и $(n,-)$ записываются как $n^{+}$и $n^{-}$соответственно.

Множество триплетов с описанными выше отношением порядка и парой операций сложения является биаддитивной решеткой. Парная ассоциативность триплетов следует из парной ассоциативности сигнатур.

КРИТЕРИЙ РЕГУЛЯРНосТИ. $x \in \mathbb{N}^{ \pm}$является регулярным әлементом, если и только если $x<+\infty u \operatorname{sgn} x=0$.

ДокаЗАтельство. Регулярность натуральных чисел очевидна. Если $\operatorname{sgn} x \neq 0$, то $2\langle+\rangle x=2^{\operatorname{sgn} x}\langle+\rangle x$, что демонстрирует нерегулярность $x$.

В силу этого критерия мы отождествляем натуральные числа с регулярными триплетами. Триплеты ненулевой сигнатуры назьваются сингулярными. Триплеты отрицательной сигнатуры назьваются минорнымми, а положительной - мажорными .

Триплеты мы обозначаем мальми греческими $\alpha, \beta, \gamma$ или малыми латинскими $x, y, z$ буквами. Буквами $k, m, n$ обозначаются натуральные числа.

Неособое вычитание. Вычитанием мы будем назьвать тройку, образованную двумя операндами (уменьшаемым и вычитаемьм) и операцией вычитания. А разностью называется результат вычитания. Вычитание $x[\langle-\rangle y$ будем называть неособым, если оба операнда конечны и разность определяется по следующим формулам неособого выччтания:

$$
\mid x[\langle-\rangle y|=| x|-| y \mid, \quad \operatorname{sgn} x[\langle-\rangle] y=\operatorname{sgn} x[\langle-\rangle] \operatorname{sgn} y .
$$

Всегда, когда формулы неособого вычитания задают триплет, этот триплет является искомой разностью. Однако эти формулыне задают триплета при $x<y$ или когда они определяют один из следующих трех некорректных триплетов: $1^{-}, 0^{+}, 0^{-}$. В этих случаях для получения значения разности нужно провести коррекцию результата. Положим corr ${ }^{+} 1^{-}=1=\operatorname{corr}^{+} 0^{+}, \operatorname{corr}^{+} 0^{-}=0$ и $\operatorname{corr}^{-} 0^{+}=0=\operatorname{corr}^{-} 1^{-}$.

В таком случае односторонние разности можно было бы выразить формулой

$$
x\left[\langle-\rangle y=\operatorname{corr}^{[\langle \pm\rangle]}(|x|-|y|, \operatorname{sgn} x[\langle-\rangle] \operatorname{sgn} y) .\right.
$$

Самое большее, что может дать плюс-коррекция из некорректного кода, - это единицу. Поэтому, если мажорная разность превышает единицу, то вычитание неособо. И самое большее, что может дать минус-коррекция из некорректного кода, - это нуль. В результате получаем следуюшее предложение. 
НЕОСОБОСТЬ НЕМАЛЫХ РАЗНОСТЕЙ. Если $x \geqslant y$, то неособо минорное вычитание $x[-] y$, а если $x>y+1$, то неособо мажсорное $x\langle-\rangle y$.

Мажорную разность мы будем назьвать малой, если она не превосходит единицы, а минорную, если она не превосходит нуля. В случае, если разность не мала, она, как показано вьше, получается неособым вычитанием. Следуюшее предложение доказывается непосредственной проверкой.

ФОРМУЛЫ МАЛЫХ РАЗНОСТЕЙ.

(1) $\alpha\langle-\rangle \beta=0$, если и только если $\alpha \leqslant \beta$;

(2) $\alpha\langle->\beta=1$, если и только если $\beta<\alpha \leqslant \beta+1$;

(3) $\alpha[-] \beta=0$, если и только если $\beta \leqslant \alpha<\beta+1$.

Будем говорить, что вычитание линейно, если основа разности равна разности основ уменьшаемого и вычитаемого. Мы также будем употреблять термин линейная разность для обозначения результата линейного вычитания. В случае малых разностей линейность может нарушаться, но всегда выполнены следуюшие неравенства.

НЕРАВЕНСТВА ПОЛУЛИНЕЙНОСТИ.

$|x\langle-\rangle| \geqslant|x|-|y|$ (мажсорная суперлинейность),

$|x[-] y| \leqslant|x|-|y|$ (минорная сублинейность).

Из этих неравенств вытекает такое соотношение между основами односторонних разностей:

$$
|x\langle-\rangle y| \geqslant|x[-] y| \text {. }
$$

Из неравенств полулинейности получаем

СлЕДСТВИЕ. Двухстороннее вычитание линейно.

КрИТЕРИЙ ДВУХстОронностИ. Если $n$ натуральное $u x \neq n$, mо $x\langle-\rangle n=$ $x[-] n$, если и только если $x \geqslant(n+1)$, и $n\langle->x=n[-] x$, если и только если $x \leqslant n-1$.

ДокАЗАТЕЛЬСТво. Если $x>n+1$, то разности $x[\langle-\rangle] n$ не малые, а потому неособые, а потому совпадают. Если $x=n+1$, то обе разности равны единице. Если $n<x<n+1$, то $x[-] n=0$ и $x\langle-\rangle n=1$. Так, доказано первое утверждение. Второе мы оставляем читателю.

Отметим, что из проведенного анализа вытекает такое следствие:

НЕОСОБОСТЬ ДВУХСТОРОННЕЙ РАЗНОСТИ. Если один из операндов вычитания натурален, то разность является двухсторонней в том и только том случае, когда минорное и мажорное вычитания неособы.

КРИТЕРИЙ МАЖОРНОГО ПОДЧИНЕНИЯ. ДЛя любого $y>1$ будет $0,1, y\langle-\rangle 1\langle<\rangle$. Если $1<x<y$, то $x\langle<\rangle y$, если и только если $x \leqslant y-1 u \operatorname{sgn} x\langle\leqslant\rangle \operatorname{sgn} y$.

ДоКАЗАТЕЛЬСТВО. Ноль и $y$ мажорно подчинены $y$ ввиду минимальности разложения $y\langle=\rangle y\langle+\rangle 0$. Подчиненность единищы вытекает из разложения $y\langle=\rangle(y\langle-\rangle 1)\langle+\rangle 1$.

Пусть теперь $1<x<y$ и $x=y\langle-\rangle z$. Тогда разность немала, вьгитание $y\langle-\rangle z$ неособо, и потому $\operatorname{sgn} x=\operatorname{sgn} y\langle-\rangle \operatorname{sgn} z$ и $\operatorname{sgn} y\langle<\rangle \operatorname{sgn} z$. Поскольку $x \neq y$, постольку 
$z \geqslant 1$. Следовательно, $x \leqslant y-1$. И необходимость сформулированного условия доказана.

Пусть теперь $1<x \leqslant y-1$ и $\operatorname{sgn} x\langle\leqslant \operatorname{sgn} y$. Тогда $y>2$. Если $x=y-1$, то $x\langle<\rangle y$. Если $x<y-1$, то $x+1<y$, и в этом случае выгитание $y\langle-\rangle x$ является неособым. В таком случае $\operatorname{sgn} x=\operatorname{sgn} y\langle-\rangle \operatorname{sgn}(y\langle-\rangle x)$ и $|x|=|y\langle-\rangle(y\langle-\rangle x)|$. Откуда $x=y\langle-\rangle(y\langle-\rangle x)$. Что и требовалось доказать.

Целые части. Для конечного триплета $x$ определим его надиелую часть $\lceil x\rceil$ как наименьшее целое число, не уступаюшее $x$, и определим подчелую часть $\lfloor x\rfloor$ как наибольшее целое число, не превосходяшее $x$. И полагаем $\lceil\infty\rceil=\lfloor\infty\rfloor=\infty$.

ФОРМУЛА ЦЕЛОЙ чАСТИ. Если $\operatorname{sgn} x=0$, mo $\lceil x\rceil=\lfloor x\rfloor=|x| ;$ ecлu $\operatorname{sgn} x=+$, $m o\lceil x\rceil=|x|+1 u\lfloor x\rfloor=|x| ;$ ecлu $\operatorname{sgn} x=-, m o\lfloor x\rfloor=|x|-1 u\lceil x\rceil=|x|$.

Таким образом, тройка чисел $\lceil x\rceil,|x|$ и $\lfloor x\rfloor$ однозначно определяет триплет.

ФОРМУЛЫ ЦЕЛОЙ ЧАСТИ СУММЫ. Для любого триплета $x$ и любого натурального п справедливы следующие соотношения:

(1) $\lceil x+n\rceil=\lceil x\rceil+n,\lfloor x+n\rfloor=\lfloor x\rfloor+n$;

(2) $\left\lceil x[+] n^{-}\right\rceil=|x|+n,\left\lfloor x\langle+\rangle n^{+}\right\rfloor=|x|+n$;

(3) $\left\lceil x[+] n^{+}\right\rceil=\lfloor x\rfloor+n+1,\left\lfloor x\langle+\rangle n^{-}\right\rfloor=\lceil x\rceil+n-1$.

ДоКАЗАТЕЛЬСтво. Ограничимся доказательством первого равенства третьего пункта. Если $x$ не минорен, то $|x|=\lfloor x\rfloor$ и мажорна сумма $x[+] n^{+}$. Откуда $\left\lceil x[+] n^{+}\right\rceil=\left|x[+] n^{+}\right|+1=|x|+n+1=\lfloor x\rfloor+n+1$. Если $x$ минорен, то минорна сумма $x[+] n^{+}$и $\lfloor x\rfloor=|x|-1$, откуда $\left\lceil x[+] n^{+}\right\rceil=\left|x[+] n^{+}\right|=|x|+n=n+\lfloor x\rfloor+1$.

ПРИНЦИПЫ ТЕСТИРОВАНИЯ. Для любой триплет $x$ однозначно определяется любой из следующих двух четверок натуральных чисел:

(1) $n,\lceil x+n\rceil,\left\lceil x[+] n^{+}\right\rceil,\left\lceil x[+] n^{-}\right\rceil$;

(2) $n,\lfloor x+n\rfloor,\left\lfloor x\langle+\rangle n^{+}\right\rfloor,\left\lfloor x\langle+\rangle n^{-}\right\rfloor$.

ДОКАЗАТЕЛЬСТВО. Действительно, в силу формул целой части суммы имеем $\lceil x\rceil=\lceil x+n\rceil-n,|x|=\left\lceil x[+] n^{-}\right\rceil-n,\lfloor x\rfloor=\left\lceil x[+] n^{+}\right\rceil-n-1$. Поэтому первая четверка определяет $x$. Аналогично доказьвается, что вторая тоже.

При любых $x, y$ справедливы такие неравенства иелой части

$$
\lfloor x\rfloor+\lfloor y\rfloor \leqslant\lfloor x\langle+\rangle y\rfloor \leqslant\lceil x\rceil+\lfloor y\rfloor \leqslant\lceil x[+] y\rceil \leqslant\lceil x\rceil+\lceil y\rceil .
$$

\section{Триплетные кодировки}

Триплетнылми последовательностями мы будем назьвать функции из $\mathfrak{P}_{0}$ в $\mathbb{N}^{ \pm}$, принимающие в нуле регулярное или бесконечное значение. Обозначать триплетные последовательности будем большими латинскими буквами $A, B, C, D \ldots$ Триплетная последовательность назьвается знакопостоянной, если либо все ее члены положительны (положительная последовательность), либо нулевые (нулевая последовательность). Триплетная последовательность $D$ называется унирегулярной, если регулярность $D(p)$ влечет равенство $D(p)=D(0)$. 
Знакопостоянная унирегулярная триплетная последовательность назьвается корректной. Корректные триплетные последовательности будем кратко назьвать кодировками. Совокупность кодировок обозначается $\mathfrak{C D}$ и называется кодовой алгеброй.

Отношение порядка на триплетах порождает отношение частичного порядка на кодировках (почленный порядок). А именно, считаем, что $D \geqslant C$, если $D(p) \geqslant C(p)$ для любого простого $p \geqslant 0$.

Операции на триплетах порождают аналогичные операции на триплетных кодировках. Для двух триплетных последовательностей $C, D$ определим их односторонние суммы $C[\langle+\rangle D$ почленно. Так что $(C[\langle+\rangle])(p)=C(p)[\langle+\rangle D(p)$. Тогда сумма знакопостоянных последовательностей - знакопостоянна, а сумма унирегулярных унирегулярна. Следовательно, эти операции, совершаемые над кодировками, дают кодировки.

Постоянная триплетная последовательность корректна в том и только том случае, когда она принимает регулярное значение. Постоянную последовательность, принимающую натуральное значение $n$, мы также обозначаем как $n$ и назьваем натуральной кодировкой.

Для произвольной положительной триплетной последовательности $C$ определим две корректные последовательности $C^{+}$и $C^{-}$, называемые соответственно плюс-коррекиией и минус-коррекиией триплетной последовательности $C$, следующим образом: $C^{+}(p)=C(p)=C^{-}(p)$, если $C(p)$ нерегулярно или $C(p)=C(0)$, иначе $C^{+}(p)=$ $C(p)^{+}$, a $C^{-}(p)=C(p)^{-}$. Ясно, что для корректной последовательности $C$ обе ее коррекции совпадают с $C$. Операции коррекции применяются при определении точньх граней.

ФОРМУЛЫ ТОЧНЫХ ГРАНЕЙ. Для любой пары кодировок $C, D$ плюс-коррекиия последовательности $\max \{C(p), D(p)\}$ является их точной верхней гранью в $\mathfrak{C} \mathfrak{D}$, а минус-коррекиия последовательности $\min \{C(p), D(p)\}$ является точной нижней.

ДоКАЗАТЕЛЬСТВО. Если $C(0)=D(0)$, то формулы $E(p)=\max \{C(p), D(p)\}$ и $F(p)=\min \{C(p), D(p)\}$ задают корректные кодировки, которые, очевидно, являются точными гранями. В случае, если $\mathrm{C}(0) \neq D(0)$, эти формулы могут дать последовательности, принимающие два регулярных значения - $E(0)$ и $F(0)$. Плюс-коррекция $E^{+}$, как следует из ее определения, почленно превосходит как $C$, так и $D$. Если же $B$ - кодировка, превосходящая $C$ и $D$, то при любом $p$ будет $B(p) \geqslant E(p)$. Если же мы предположим, что $B(p)<E^{+}(p)$, то $B(p)=E(p)$ регулярно и $E(p) \neq E(0)$, поэтому $E(p)=F(0)<E(0)$. В силу унирегулярности $B$ имеем $B(p)=B(0)$, откуда $B(0)<E(0)$, что противоречит условию $B \geqslant E$. Аналогично разбирается случай нижней грани.

ЛЕмма о ДИСТРИБУтивностИ. Для любых трех кодировок имеем:

$$
C[+] \sup \{A, B\}=\sup \{C[+] A, C[+] B\}, \quad C\langle+\rangle \inf \{A, B\}=\inf \{C\langle+\rangle A, C\langle+\rangle B\} .
$$

ДоКАЗАТЕЛЬСТВО. Пусть $E(p)=\max \{A(p), B(p)\}$ - почленньй максимум кодировок $A, B$. Тогда левая часть равна $C[+] E^{+}$, а правая равна $(C[+] E)^{+}$. Если для некоторого $p$ имеем $E^{+}(p)=E(p)$, то или $E(p)$ нерегулярно и тогда нерегулярно 
$E(p)[+] C(p)$, или $E(p) \neq E(0)$ и тогда $E(p)[+] C(p) \neq E(0)+C(0)$, т.е. в обоих случаях имеем $(C[+] E)^{+}(p)=(C[+] E)(p)$. И потому левая часть равна правой. В противном случае $E(p)$ регулярно и отлично от $E(0)$. Тогда слева получается $E(p)^{+}[+] C(p)$. Если $C(p)$ регулярно, то $C(p)$ [+] $E(p)$ также регулярно и отлично от $C(0)+E(0)$, поэтому значение правой части будет $(E(p)+C(p))^{+}$, что совпадает со значением левой. Если же $C(p)$ нерегулярно, то получим $\left.E(p)^{+}{ }^{+}+\right] C(p)=E(p)[+] C(p)=(E[+] C)^{+}(p)$, что и требовалось доказать. Доказательство мажорной дистрибутивности аналогично.

ЛЕмМа о НАПРАВЛЕННостИ. Пусть $D_{i}$ - направленное множество кодировок. Тогда если $D_{i}$ направленно по возрастанию, то $D(p)=\sup D_{i}(p)$ является корректной кодировкой и $D=\sup D_{i}$, а если по убьванию, то $D(p)=\inf D_{i}(p)$ является корректной кодировкой и $D=\inf D_{i}$.

ДокАЗАТЕЛЬСТво. Пусть $D_{i}$ направленно по возрастанию. Если одно из $D_{i}$ положительно, то $D(p)$ также положительно. Пусть $D(p) \neq D(0)$ конечны, тогда найдется $i$, для которого $D_{i}(0)=D(0)$, и найдется $j$, для которого $D_{j}(p)=D(p)$. Пусть $k$ такое, что $D_{k} \geqslant D_{i}, D_{j}$. Тогда $D_{k}(p)=D(p)$ и $D_{k}(0)=D(0)$. Ввиду унирегулярности $D_{k}$ получаем, что $D_{k}(p)=D(p)$ нерегулярно, что доказывает унирегулярность $D$. Случай убьваюшей направленности разбирается аналогично.

Теорема. Множество кодировок с определенной выше парой сумм является полной биаддитивной решеткой, ненулевьми регулярными әлементами которой являются натуральные кодировки.

ДокАЗАТЕЛЬСТво. Пусть $C_{i}, i \in I,-$ произвольное множество кодировок. Тогда для любого конечного $J \subset I$ положим $C_{J}=\sup _{i \in J} C_{i}$ (супремум сушествует в соответствии с формулой точных граней). Тогда множество супремумов конечных подмножеств, будучи направленным по возрастанию, имеет супремум, согласно лемме о направленности. И этот супремум и будет супремумом множества $C_{i}$. Тем самым установлено, что $\mathfrak{C D}$ является полной решеткой.

Коммутативность и ассоциативность операций сложения кодировок вытекает из аналогичных свойств триплетов, ведь эти операции вьполняются почленно. Ковариантность операций порядку очевидна по этой же причине. Дистрибутивность мажорного сложения по отношению к супремуму для конечных множеств вытекает из леммы о дистрибутивности, а для бесконечных вьводится из конечного результата с помошью леммы о направленности в духе проведенного вьше доказательства полноты.

Нейтральной по отношению к обеим операциям является нулевая кодировка. А свойство парной ассоциативности кодировок вытекает из этого свойства триплетов в силу почленной определенности операций сложения и неравенств.

Регулярность натуральных кодировок очевидна. Проверку того, что никакая ненатуральная кодировка регулярной не является, мы оставляем читателю и в дальнейшем этот факт не используем.

В дальнейшем нам потребуется еще такое свойство кодовой решетки, которое мы будем назьвать счетной теснотой.

Счетная ТЕСНОТА КОДИРОвОК. Всякое множество кодировок содержит счетное подмножество, которое имеет такой же супремум и инфимум. 
ДоКАЗАТЕЛЬСТвО. Поскольку множество имеет такой же супремум (инфимум) как направленное множество, образованное супремумами (инфимумами) его конечных подмножеств, доказательство достаточно провести для направленных множеств. Пусть $\Delta$ - некоторое несчетное направленное множество кодировок, тогда его верхняя и нижняя грани согласно лемме о направленности вычисляются почленно. Для всякого $p$ существует счетное множество $\Delta_{p} \subset \Delta$, для которого супремум и инфимум множества значений в $p$ такие же, как у всего $\Delta$. В таком случае $\bigcup_{p \in \mathfrak{P}_{0}} \Delta_{p}$ будет счетным множеством с такими же супремумом и инфимумом, что и $\Delta$.

\section{Разности кодировок}

Разности кодировок, в отличие от сумм, не всегда определяются почленно. Справедливы лишь следуюшие сравнения.

ПочЛЕННЫЕ СРАВНЕНИЯ. Для любых кодировок $C, D$ при любом простом $p$ справедливы неравенства $(D\langle-\rangle C)(p) \geqslant D(p)\langle-\rangle C(p),(D[-] C)(p) \leqslant D(p)[-] C(p)$.

ДокАЗАТЕЛЬСТВО. Поскольку $C\langle+\rangle(D\langle-\rangle C) \geqslant D$, постольку при любом $p$ имеем $C(p)\langle+\rangle(D\langle-\rangle C)(p) \geqslant D(p)$. Перенося $C(p)$ в правую часть, получаем нужное мажорное неравенство. Минорное доказьвается аналогично.

Почленная разность $D(p)$ [<-〉] $C(p)$ нередко оказьвается некорректной последовательностью. В тех же случаях, когда она корректна, она и является разностью $C[\langle-\rangle] D$.

ЛЕММА О КОРРЕКТНОЙ РАЗНОСТИ. Пусть $C$ и $D-$ две кодировки. Тогда если $B(p)=D(p)[\langle-\rangle C(p)$ является кодировкой, то $B=D[\langle-\rangle C$.

ДокАЗАТЕЛЬСтво. Ограничимся рассмотрением мажорного случая. Очевидно неравенство $B\langle+\rangle C \geqslant D$. Откуда $B \geqslant D\langle-\rangle C$. Обратное неравенство доказано вьше.

Важньй частньй случай, когда почленная разность корректна, представлен следующим предложением.

ПОЧЛЕННОСТЬ НЕМАЛОЙ РАЗНОСТИ. Если разность $C(p)[\langle-\rangle D(p)$ не является малой ни для какого $p$, то $(C[\langle-\rangle D)(p)=C(p)[\langle-\rangle] D(p)$.

ДокАЗАТЕльство. Если $C(p)[\langle-\rangle D(p)$ не является малой, то она неособа и ее регулярность означает одновременную регулярность $C(p)$ и $D(p)$. Поскольку $C$ и $D$ унирегулярны, отсюда следует, что $C(p)=C(0)$ и $D(p)=D(0)$. Откуда $C(p)-D(p)=C(0)-D(0)$. Тем самым и доказано, что почленная разность дает корректную кодировку.

ПОчЛЕННОСТЬ ДВУХСТОРОННЕЙ РАЗНОСТИ. Если $D\langle-\rangle C=D[-] C, \quad m o$ $(D-C)(p)=D(p)-C(p)$ для всякого $p$.

ДокАЗАТЕльство. Поскольку $D\langle-\rangle C \geqslant 0$, постольку и $D[-] C \geqslant 0$, а следовательно, $D \geqslant C$. Если $D=C$, то обе разности нулевые и почленные. Если $D>C$, то $D\langle-\rangle C>0$, и потому $D\langle-\rangle C \geqslant 1$. Откуда $D[-] C \geqslant 1$. Следовательно, $D \geqslant C+1$. Но в таком случае минорное вычитание $D(p)[-] C(p)$ немалое при любом $p$, и, следовательно, минорная разность $D[-] C$ почленна. Поэтому $D\langle->C$ совпадает с почленной 
минорной разностью. Но почленная мажорная разность, с одной стороны, не меньше почленной минорной, а с другой - не больше мажорной. Откудаполучаем почленность мажорной разности. Что и требовалось доказать.

КРИТЕРИЙ ДВУХСТОРОНнОсТИ. Пусть $n-$ натуральная кодировка. Если $D \geqslant$ $n+1$, и только в этом случае, $D\langle->n=D[-] n$. Eсли $D \leqslant n-1$, и только в этом случае, $n\langle-\rangle D=n[-] D$.

ДокАЗАТЕльство. Ограничимся доказательством первого утверждения. Если $D \geqslant n+1$, то согласно доказанному в параграфее о триплетах критерию двухсторонности разности при любом $p$ разность $D(p)-n$ двухсторонняя и неособая. Поэтому ее регулярность влечет регулярность $D(p)$, а потому и равенство $D(p)-n=D(0)-n$. Поэтому $D(p)-n$ унирегулярна и корректна. Поэтому двухсторонность разности $D-n$ вытекает из леммы о корректной разности. Если же разность $D-n$ двухсторонняя, то она почленная. И неравенство $D(p) \geqslant n+1$ следует теперь из триплетного критерия двухсторонности разности.

КРИТЕРИЙ ПОДЧИНЕНИЯ. Кодировка $D$ мажсорно подчинена натуральной $n$, если и только если $D \leqslant n-1$.

ДокаЗАТЕЛЬСТво. Если $D \leqslant n-1$, то $n\langle-\rangle D=n[-] D$ и $(n-D)(p)=n-D(p)$ согласно вышедоказанному. Если $D=0$, то $n\langle-\rangle(n-D)=n\langle-\rangle n=0=D$. Если $D>0$, то $n-D \leqslant n-1$, и потому разность между $n$ и $n-D$ двухсторонняя и почленно определенная. Поэтому $(n-(n-D))(p)=n-(n-D(p))=D(p)$ в силу критерия подчинения триплетов. Итак, если $D \leqslant n-1$, то $D\langle<\rangle n$. Если же $D=n\langle-\rangle(n\langle-\rangle D)$, и $D \neq n$, то $(n\langle-\rangle D) \geqslant 1$, и потому $D \leqslant n\langle-\rangle 1=n-1$. Что и требовалось доказать.

\section{Тестирование}

Нормы кодировок. Наименьшее натуральное число $n$, для которого $n \geqslant D$, называется верхней нормой триплетной последовательности $D$ или ее размерностью и обозначается $\|D\|$. Отметим, что существует единственная кодировка единичной размерности, и она тождественно равна единице.

Наибольшее целое число $n$, для которого $n \leqslant D$, назьвается нижней нормой триплетной последовательности $D$ или ее связностью и обозначается $\langle\langle D\rangle\rangle$. Таким образом, равенство $\|D\|=\langle\langle D\rangle\rangle$ характеризует постоянную последовательность. Верхняя и нижняя норма могут быть выражены соотношениями:

$$
\|D\|=\max \lceil D(p)\rceil, \quad\langle\langle D\rangle\rangle=\min \lfloor D(p)\rfloor .
$$

Следующие оценки норм суммы триплетных последовательностей вытекают из триплетных неравенств для целых частей

$$
\begin{gathered}
\langle\langle D\rangle\rangle_{1}+\left\|D_{2}\right\| \leqslant\left\|D_{1}+D_{2}\right\| \leqslant\left\|D_{1}\right\|+\left\|D_{2}\right\|, \\
\langle\langle D\rangle\rangle_{1}+\langle\langle D\rangle\rangle_{2} \leqslant\left\langle\left\langle D_{1}+D_{2}\right\rangle\right\rangle \leqslant\left\|D_{1}\right\|+\langle\langle D\rangle\rangle_{2} .
\end{gathered}
$$


Экстремальные кодировки. Ввиду полноты решетки кодировок среди всех кодировок, принимающих при данном $p$ значение $\alpha$, найдутся как наименьшая, так и наибольшая. Наименьшую назьваем $(\alpha, p)$-минимальной и обозначаем $M^{(\alpha, p)}$, наибольшую назьваем $(\alpha, p)$-максимальной и обозначаем $M_{(\alpha, p)}$ Непосредственно из определения экстремальных кодировок вытекают такие формуль разложения

$$
D=\sup _{p \geqslant 0} M^{(D(p), p)}=\inf _{p \geqslant 0} M_{(D(p), p)} .
$$

СвОЙСТВА МИНИМАЛЬНОГО КОДА. Пусть $M=M^{(n, p)}$, mогда:

(1) $|M(q)|=1$ для любого $q \neq p, 0$;

(2) если $\alpha$ сингулярно, то $M(q)=1$ для всех $q \neq p$;

(3) если а регулярно, то $M(0)=M(p)$.

ДоКАЗАТЕЛЬСТво. Если $|M(q)|>1$, то, изменив значение $M(q)$ на $1^{+}$в случае $q \neq p, 0$, получим корректную кодировку, опровергающую минимальность $M(q)$. Таким образом, первый пункт доказан. Если $\alpha$ сингулярно, то корректной является кодировка, равная единице всюду, за исключением $p$, где она равна $\alpha$, поэтому $M$ не может иметь больших значений. А меньше - только нули. Наконец, если $\alpha$ регулярно, то $M(0)=\alpha$ в силу корректности кода.

СВОЙСТВА МАКСИМАЛЬНОГО КОДА. Пусть $M=M_{(n, p)}$, moгда:

(1) $|M(q)|=+\infty$ для любого $q \neq p, 0$;

(2) если $\alpha$ сингулярно, то $M(q)=+\infty$ для всех $q \neq p$;

(3) если а регулярно, то $M(0)=M(p)$.

ДоКАЗАТЕльство предоставляется читателю.

ФоРМУЛЫ ДОБАВЛЕНИЯ МИНИМАЛЬНЫХ КОДИРОВОК. $E c \Omega u\|D\|<n, m o$ :

(1) $\left\|D[+] M^{(n, p)}\right\|=\max \{\lceil D(p)\rceil, D(0)\}+n$;

(2) $\left\|D[+] M^{\left(n^{+}, p\right)}\right\|=\lfloor D(p)\rfloor+n+1$;

(3) $\left\|D[+] M^{\left(n^{-}, p\right)}\right\|=|D(p)|+n$.

ДоКАЗАТЕЛЬСТВо. Если $\|D\|<n$, то $n \geqslant|D(q)|+1=\left|D(q)[+] M^{(n, p)}(q)\right|$ при любом $q \neq p, 0$. И, с другой стороны, если $\|D\|>0$, то $\left|D(p)[+] M^{(n, p)}(p)\right| \geqslant 1+n$. Поэтому $D[+] M^{(n, p)}$ принимает максимальное значение в $p$ или нуле, а в остальных случаях в $p$, и нужные нам равенства вытекают из триплетных формул для целой части суммы.

ФОРМУЛЫ ДОБАВЛЕНИЯ МАКСИМАЛЬНЫХ КОДИРОВОК. Для любой $D$ имеем:

(1) $\left\langle\left\langle D\langle+\rangle M_{(n, p)}\right\rangle\right\rangle=\min \{\lfloor D(p)\rfloor, D(0)\}+n$;

(2) $\left\langle\left\langle D\langle+\rangle M_{\left(n^{-}, p\right)}\right\rangle\right\rangle=\lceil D(p)\rceil+n-1$;

(3) $\left\langle\left\langle D\langle+\rangle M_{\left(n^{+}, p\right)}\right\rangle\right\rangle=|D(p)|+n$.

ДоКАЗАТЕльСТВо предоставляется читателю.

Итог нашим рассмотрениям подводит пара теорем. 
ТЕОРема о РАЗМЕРНОСТНОм ТЕСТИРОВАНИИ. Для совпадения конечномерных кодировок $C$ и $D$ необходимо и достаточно, чтобы равенство $\|C[+] B\|=$ $\|D[+] B\|$ выполнялось для любой кодировки $B$.

ДокАЗАТЕЛЬСТво. Необходимость условия очевидна, докажем достаточность. Равенство $\left\|C[+] M^{(n, 0)}\right\|=\left\|D[+] M^{(n, p)}\right\|$ позволяет, в силу формул добавления минимальных кодировок, получить $C(0)=D(0)$. Если $C(p)>C(0)$, то $\lceil C(p)\rceil>C(0)$, и потому $\left\|C[+] M^{(n, p)}\right\|>n+C(0)$, поэтому и $\left\|D[+] M^{(n, p)}\right\|>n+D(0)$, откуда $D(p)>D(0)$ и $\lceil D(p)\rceil=\lceil C(p)\rceil$. Сложение с минимальньми кодировками других типов дает равенства $|D(p)|=|C(p)|$ и $\lfloor D(p)\rfloor=\lfloor C(p)\rfloor$. Поэтому $C(p)=D(p)$, если одно из них больше чем $C(0)=D(0)$. Пусть теперь $C(p), D(p)<C(0)$. В этом случае $C(p)$ и $D(p)$ сингулярны. Их основы совпадают и для заключения о совпадении их сигнатур достаточно равенства подщелых частей. Если $C(p)=C(0)$, то $D(p) \leqslant D(0)$ и $|D(p)|=|C(p)|=\lfloor C(p)\rfloor=\lfloor D(p)\rfloor$, откуда следует неминорность $D(p)$ и вместе с ней равенство $D(p)=D(0)=C(p)$. Теорема полностью доказана.

ТЕОРема о СВЯЗНОСТНОм ТЕСТИРОВАНИИ. Для совпадения кодировок $C u$ $D$ необходимо и достаточно, чтобы при любом $B$ выполнялось равенство $\langle\langle C\langle+\rangle B\rangle\rangle=\langle\langle D\langle+\rangle B\rangle\rangle$.

ДоКАЗАТЕЛЬСТВо предоставляется читателю.

\section{Кодирование}

Прежде чем кодировать полигрупш, кодируем бокштейновские группы. Положим $\operatorname{sgn}\left(\mathbb{Z}_{(p)}\right)=0, \operatorname{sgn}\left(\mathbb{Z}_{p}\right)=+$ и $\operatorname{sgn}\left(\mathbb{Q}_{p}\right)=-$.

Пусть $\mathscr{G}$ - некоторая верхняя полигруппа. Поставим ей в соответствие кодировку Alt $\mathscr{G} \in \mathfrak{C D}$, полагая $|\operatorname{Alt} \mathscr{G}(p)|=\operatorname{alt}_{p} \mathscr{G}$ и $\operatorname{sgn} \operatorname{Alt} \mathscr{G}(p)=-\operatorname{sgn}\left(\left|\sigma_{p}^{+} \mathscr{G}\right|\right)$ для любого простого $p \geqslant 0$.

Всякой нижней полигруппе $\mathscr{G}$ поставим в соответствие кодировку Dep $\mathscr{G} \in \mathfrak{C} \mathfrak{D}$, полагая $|\operatorname{Dep} \mathscr{G}(n)|=\operatorname{dep}_{p} \mathscr{G}$ и $\operatorname{sgn} \operatorname{Dep} \mathscr{G}(p)=\operatorname{sgn}\left(\left|\sigma_{p}^{-} \mathscr{G}\right|\right)$ для любого простого $p \geqslant 0$. Кодировкой нулевой полигруппы считается постоянная, принимающая значение $+\infty$. Кодировки Alt $\mathscr{G}$ и Dep $\mathscr{G}$ назьваются факторными кодировками полигрупп.

Отображение $f$ частично упорядоченных множеств назьвается изотонным, если оно инъективно и неравенство $x \leqslant y$ равносильно неравенству $f(x) \leqslant f(y)$ для любых $x, y$.

Основньм результатом этого параграф̆а является следующая теорема.

ТЕОРЕМА О КОДИРОВАНИИ ПОЛИГРУПП.

(1) Alt: $\mathfrak{K}^{*} \rightarrow \mathfrak{C D}$ изотонно отображает $\mathfrak{K}^{*}$ на множество кодировок конечной размерности.

(2) Dep: $\mathfrak{K}_{*} \rightarrow \mathfrak{C D}$ изотонно отображсает $\mathfrak{K}_{*}$ на множсество ненулевых кодировок.

(3) Alt $\mathscr{G}[\times] \mathscr{H}=$ Alt $\mathscr{G}[+]$ Alt $\mathscr{H}$ для любьх верхних полигрупп.

(4) $\operatorname{Dep} \mathscr{G}\langle\times\rangle \mathscr{H}=\operatorname{Dep} \mathscr{G}\langle+\rangle$ Dep $\mathscr{H}$ для любых нижних полигрупп.

(5) alt $\mathscr{G}=\|$ Alt $\mathscr{G} \|$ для любой верхней полигруппьи $\mathscr{G}$.

(6) $\operatorname{dep} \mathscr{H}=\langle\langle\operatorname{Dep} \mathscr{H}\rangle\rangle$ для любой нижней полигруппь $\mathscr{H}$.

(7) $\mathscr{G} \tau \mathscr{H} \Longleftrightarrow$ Alt $\mathscr{G} \leqslant$ Dep $\mathscr{H}$ для любых верхней $\mathscr{G}$ и нижней $\mathscr{H}$ полигрупп. 
ДокАЗАТЕЛЬство. Для конечномерной кодировки $D$ определим верхнюю полигруппу $\sigma(D)$ как сумму $\sigma(D)=\bigoplus_{p \geqslant 0} \sigma_{p}(D)$, где $\sigma_{p}(D)=\mathbb{Z}_{(p)}^{[|D(p)|]}$, ecли $\operatorname{sgn} D(p)=0$, $\sigma_{p}(D)=\mathbb{Z}_{p}^{[|D(p)|]}$, если $\operatorname{sgn} D(p)=-$ и $\sigma_{p}(D)=\mathbb{Q}_{p}^{[|D(p)|+1]}$, если $\operatorname{sgn} D(p)=+$.

Тогда, как нетрудно проверить с помощью формулы для $p$-высоты, имеет место равенство $\sigma_{p}(\sigma \operatorname{Alt} \mathscr{G})=\sigma_{p}^{+} \mathscr{G}$. Откуда следует, что $\operatorname{ALT}(\sigma \operatorname{Alt} \mathscr{G})=\operatorname{ALT} \mathscr{G}$. И нетрудно проверить также, что Alt $\sigma D=D$ для любой кодировки. Таким образом, отображения $\mathscr{G} \rightarrow \operatorname{Alt} \mathscr{G}$ и $D \rightarrow \sigma D$ являются взаимно обратньми биекциями между верхней алгеброй Кюннета и конечномерной кодовой решеткой.

Условие сохранения порядка, в силу критерия факторов, достаточно проверить для бокштейновских моногруп. Все возможные значения $p$-фактора Бокштейна представляют собой линейно упорядоченное множество моногрупп, которое в порядке возрастания выстраивается в следующую последовательность: $\mathbb{Z}_{(p)}^{[0]}, \mathbb{Z}_{(p)}^{[1]}, \mathbb{Q}_{p}^{[2]}, \mathbb{Z}_{p}^{[2]}$, $\mathbb{Z}_{(p)}^{[2]}, \mathbb{Q}_{p}^{[3]}, \mathbb{Z}_{p}^{[3]}, \mathbb{Z}_{(p)}^{[3]}, \ldots$ Эта последовательность посредством Alt переводится в выстроенную в порядке возрастания последовательность триплетов $0,1,1^{+}, 2^{-}, 2$, $2^{+}, 3^{-}, 3, \ldots$. То есть порядок сохраняется. Таким образом, доказан первьй пункт теоремы.

Второй доказьвается аналогично. При этом формула для отображения, обратного к Dep, такая же, как для обратного к Alt, только иначе определяется значение $\sigma_{p} D$ в случае, если $\operatorname{sgn} D(p) \neq 0$, а именно, $\sigma_{p} D=\mathbb{Z}_{p}^{[|D(p)|]}$, если $\operatorname{sgn} D(p)=+$, и $\sigma_{p}(D)=$ $\mathbb{Q}_{p}^{[|D(p)|-1]}$, если $\operatorname{sgn} D(p)=-$.

Равенство Alt $\mathscr{G} \mathscr{H}=$ Alt $\mathscr{G}[+]$ Alt $\mathscr{H}$ вьводится из теоремы о факторе произведения перебором всех вариантов факторов сомножителей. То же самое относится к равенству $\operatorname{Dep} \mathscr{G} \mathscr{H}=\operatorname{Dep} \mathscr{G}\langle+\rangle \operatorname{Dep} \mathscr{H}$.

Докажем теперь последний пункт теоремы - триплетньй критерий совместимости. Пусть Alt $\mathscr{G} \leqslant$ Dep $\mathscr{H}$. Согласно факторному критерию совместимости нужньй результат вытекает из следующей леммы.

ЛЕмма. Верхняя и нижняя бокштейновские моногруппь $\mathscr{G}$ и $\mathscr{H}$ одинаковой факторности совместимы тогда и только тогда, когда $\operatorname{Alt} \mathscr{G} \leqslant \operatorname{Dep} \mathscr{H}$.

ДокаЗАТЕльство. Рассмотрим все возможные случаи. Первый случай. Пусть $\operatorname{Alt} \mathscr{G}(p)=n$ регулярно, тогда $G_{n}=\mathbb{Z}_{(p)}$. Если Dep $\mathscr{H}(p)=n$, то $H_{m}=0$ при $m<n$, и условие совместимости вьполняется. Если же $\operatorname{Dep} \mathscr{H}(p)=n^{-}$, то $H_{n-1}=\mathbb{Q}_{p}$ и $G_{n} \otimes H_{n-1} \neq 0$, т.е. совместимости нет. Таким образом, минимальное значение Dep $\mathscr{H}$, при котором имеется совместимость $\mathscr{G} \tau \mathscr{H}$, равно в этом случае $n$, т.е. совпадает с Alt $\mathscr{G}(p)$.

Второй случай. Пусть теперь $\operatorname{Alt} \mathscr{G}(p)=n^{-}$. Тогда $G_{n}=\mathbb{Z}_{p}$. Если $\operatorname{Dep} \mathscr{H}(p)=$ $n^{-}$, то $H_{n-1}=\mathbb{Q}_{p}$. Тогда условия совместимости вьполнены, ибо $\mathbb{Z}_{p} \otimes \mathbb{Q}_{p}=0$. Если же $\operatorname{Dep} \mathscr{H}(p)=(n-1)^{-}$, то $H_{n-1}=\mathbb{Z}_{p}$, и условие совместимости не вьполнено, ибо $G_{n} \otimes H_{n-1} \neq 0$.

Последний случай $\operatorname{Alt} \mathscr{G}(p)=n^{+}$. Тогда $G_{n+1}=\mathbb{Q}_{p}$. Если $\operatorname{Dep} \mathscr{H}(p)=n^{+}$, то $H_{n}=\mathbb{Z}_{p}$. Здесь совместимость имеется. Если же Dер $\mathscr{H}(p)=n$, то $H_{n}=\mathbb{Z}_{(p)}$, и неравенство нулю произведения $G_{n+1} \otimes H_{n}$ приводит к несовместимости $\mathscr{G}$ и $\mathscr{H}$. Что и завершает доказательство леммы. 


\section{Коэффициентные нормы}

Пусть теперь $G$ - некоторая абелева группа. Определим верхнюю $G$-норму $\|D\|_{G}$ кодировки $D$ как $G$-высоту полигруппы, факторная кодировка которой совпадает с $D$. Непосредственно из этого определения, в силу коэффициентной монотонности, получаем следующее свойство:

Монотонность $G$-нормы. $E с л и ~ C \leqslant D, m o\|C\|_{G} \leqslant\|D\|_{G}$.

Сопоставление правил кодирования с формулами коэффициентных высот из второго параграфа дает следующие результаты.

Формулы основных $G$-норм. Для любой $D$ справедливы равенства:

(1) $\|D\|_{\mathbb{Q}}=D(0)$;

(2) $\|D\|_{\mathbb{Z}_{p}}=|D(p)|$;

(3) $\|D\|_{\mathbb{Q}_{p}}=\lfloor D(p)\rfloor$;

(4) $\|D\|_{\mathbb{Z}_{(p)}}=\max \{\lceil D(p)\rceil, D(0)\}$.

Теорема КУЗЬмИНОВА. Для всякой абелевой группь $G$ и всякого натурального $n>1$ существует такая кодировка $T_{G}^{n}$ размерности $n$ (называемая пробной), что для любой кодировки $D$ положительной размерности $\leqslant n$ выполнено равенство:

$$
\left\|D[+] T_{G}^{n}\right\|=n+\|D\|_{G} .
$$

ДокАЗАТЕльство. Пусть сначала $G$ принадлежит системе Бокштейна. Из формул сложения с минимальньми кодами и приведенных вьше формул основных $G$-норм получаются следуюшие результаты. $T_{\mathbb{Z}_{(p)}}^{n}=M^{(n, p)}, T_{\mathbb{Z}_{p}}^{n}=M^{\left(n^{-}, p\right)}$, $T_{\mathbb{Q}_{p}}^{n}=M^{\left((n-1)^{+}, p\right)}$ и $T_{\mathbb{Q}}^{n}=M^{(n, 0)}$.

Наконец, для произвольной группы $G$ пробная кодировка $T_{G}^{n}$ определяется как $\sup \left\{T_{\sigma_{p} G}^{n} \mid p \geqslant 0\right\}$. Тогда при любом $p \geqslant 0$ имеем $T_{G}^{n}(p)[+] D(p)=T_{\sigma_{p} G}^{n}[+] D(p)=$ $n+\|D\|_{\sigma_{p} G}$, и потому $\left\|D+T_{G}^{n}\right\|=n+\max _{p \geqslant 0}\|D\|_{\sigma_{p} G}=n+\|D\|_{G}$, что и требовалось доказать.

\section{Глава 2. Топология}

\section{$\S$ 4. Теория экстмерности}

В этом параграфе пространства по умолчанию сепарабельны и метризуемы, а комплексы счетны и метризуемы. Поскольку всякий счетньй $\mathrm{CW}$-комплекс гомотопически эквивалентен метризуемуму, а возможность продолжения отображений определяется гомотопическим типом, постольку предположение метризуемости рассматриваемых комплексов не уменьшает обшности полученных результатов.

ТЕОРЕМА ОБ ЭКСТМЕРНОСТИ КОМПОНЕНТ. Если любая компонента связности компакта $X$ әкстенсивна по отношению $к$ комплексу $K$, то и сам компакт также $K$-экстенсивен. 
ДокАЗАТЕльство. Пусть дано частичное отображение $f$ компакта $X$ в $K$. Для любой компоненты $C$ зафиксируем продолжение $f_{C}$ отображения $f$ на окрестность $O C$ этой компоненты. Не ограничивая общности, можем предполагать $O C$ открыто-замкнутой. Пусть $O C_{1}, O C_{2}, \ldots, O C_{n}$ образуют покрытие $X$. Тогда искомое продолжение $f$ на $X$ можно построить, полагая $f(x)$ равной $f_{C_{i}}(x)$, где $i$ - наименьшее, для которого $x \in O C_{i}$.

ТЕОРема СЧеТНОЙ СУММЫ [20]. Если пространство Х представлено в виде оббединения счетного числа замкнутых подпространств $X_{i}$, әкстенсивных по отношению к некоторому полиэдру $L$, то и $X \tau L$.

ДоказАТЕльство. Пусть задано отображение $f: A \rightarrow L$ замкнутого подмножества $A \subset X$. Тогда в силу свойства $X_{1} \tau L$ его можно распространить на $X_{1}$. Ввиду того, что $L$ является абсолютным окрестностньм ретрактом, отображение можно распространить также на некоторую замкнутую окрестность объединения, обозначаемую $Y_{1}$. Тогда $Y_{1}$ содержит во внутренности объединение $A \cup X_{1}$, и имеется отображение $f_{1}: Y_{1} \rightarrow L$, продолжающее $f$. Применяя ту же процедуру к $f_{1}$ и $X_{2}$, строим замкнутую окрестность $Y_{2}$ объединения $Y_{1} \cup X_{2}$ и отображение $f_{2}: Y_{2} \rightarrow L$, продолжающее $f_{1}$. Продолжая процесс далее, строим последовательность замкнутых множеств $Y_{i}$ и их отображений $f_{i}$ так, что $Y_{i+1} \supset Y_{i} \cup X_{i}$ и $f_{i+1}$ продолжает $f_{i}$. Тогда совокупность $f_{i}$ определяет некоторое отображение $F$ из $X$ в $L$, продолжающее $f$, а его непрерывность доказьвается так. Если $x \in X$, то $x \in X_{k}$ для некоторого $k$. Следовательно, некоторая окрестность $O x$ принадлежит $Y_{k+1}$. Но в таком случае $F$ совпадает на $O x$ с $f_{k+1}$, которое непрерьвно. Значит и $F$ непрерывно в $x$.

$\varepsilon$-непрерывность. Для отображения $f$ его колебание в точке $x$ обозначается $\operatorname{osc}_{x} f$ и определяется как предел диаметров образов $\varepsilon$-окрестностей точки $x$ при $\varepsilon$, стремящемся к нулю. Через osc $f$ обозначается верхняя грань колебаний функщии $f$ по всем точкам ее области определения.

Функция, имеюшая колебание $<\varepsilon$, называется $\varepsilon$-непрерывной.

Гомотопическим диаметром метрики на некотором пространстве $M$ назовем нижнюю грань тех $\varepsilon$, для которых существуют $\varepsilon$-близкие негомотопные отображения в $M$ какого-либо пространства. Гомотопическим радиусом метрики, имеющей положительньй гомотопический диаметр, назовем верхнюю грань таких $\varepsilon$, что любое $\varepsilon$-непрерывное отображение в пространство $M$ находится на расстоянии, меньшем трети гомотопического диаметра $M$, от некоторого непрерывного отображения.

ЛЕМмА О МЕТРИКЕ. На всяком локально-компактном метризуемом ANRпространстве $K$ существует согласованная с топологией метрика $\rho$ положительного гомотопического радиуса.

ДокАЗАТЕЛЬСТво. Зафиксируем вложение $K$ в гильбертово пространство и ретракцию $r: O K \rightarrow K$ некоторой его окрестности. Выберем столь мелкое локально конечное открытое покрытие $\omega=\left\{U_{i}\right\}_{i \in I}$ пространства $K$, чтобы всякий его элемент $U_{i}$ содержался в $O K$ вместе со своей вьпуклой оболочкой и имел компактное замькание. Пусть $\varphi_{i}$ - подчиненное этому покрытию разбиение единицы. Пусть dist - метрика, индуцированная из гильбертова пространства. Рассмотрим метрику $\rho$, определяемую формулой $\rho(x, y)=\operatorname{dist}(x, y)+\sum_{i \in I}\left|\varphi_{i}(x)-\varphi_{i} y\right|$. Гомотопический 
диаметр этой метрики не меньше единицы. Действительно, если две точки находятся на $\rho$-расстоянии меньше чем 1 , то они принадлежат одному и тому же элементу покрытия, и потому отрезок, соединяющий их, лежит в $O K$, и, ретрагируя его на $K$, получаем путь, соединяющий эти точки и непрерывно от них зависяший. Для вьполнения второго условия выберем столь мелкое локально конечное покрытие $\omega^{\prime}=\left\{V_{a}\right\}_{a \in A}$, звездно вписанное в $\omega$, чтобы образ вьпуклой оболочки любой звезды $S t\left(x, \omega^{\prime}\right)$ при ретракщии $r$ имел $\rho$-диаметр меншше чем $1 / 4$. Пусть $-\psi_{a}$ подчиненное $\omega^{\prime}$ разбиение единицы. Определим метрику $\rho^{\prime}$, полагая $\rho^{\prime}(x, y)=\rho(x, y)+\frac{1}{4} \sum_{a \in A}\left|\psi_{i}(x)-\psi_{i} y\right|$. Тогда гомотопический диаметр $\rho^{\prime}$ также больше единицы и всякое множество $\rho^{\prime}$-диаметра меньшего $1 / 4$ содержится в какой-то $\omega^{\prime}$-звезде.

Пусть $f: X \rightarrow M$ - какое-то 1/6-непрерьвное отображение относительно $\rho^{\prime}$. Для точки $x \in X$ обозначим через $[f](x)$ пересечение замьканий образов $\bigcap[f(U)]$, где $U$ пробегает все окрестности точки $x$. Многозначное отображение $[f]$ полунепрерьвно сверху, и образы точек у этого отображения компактны и имеют диаметр $<1 / 6$. Вьпуклую оболочку компакта $[f](x)$ обозначим через $\langle f\rangle(x)$. Тогда вьпуклозначное отображение $\langle f\rangle$ также полунепрерьвно сверху, имеет диаметры $r$-образов меньше чем $1 / 6$ и по теореме фон Неймана как угодно близко аппроксимируется непрерьвными однозначными отображениями. Пусть $F$ - столь близкая его аппроксимация, что $r F(x)$ лежит на $\rho^{\prime}$-расстоянии от $r(\langle f\rangle(x))$, меньшем $1 / 6$. Тогда $\rho^{\prime}(F(x), f(x))<1 / 6+$ $1 / 6=1 / 3$. Таким образом, гомотопический радиус $\rho^{\prime}$ больше либо равен $1 / 4$. И лемма полностью доказана.

ЛЕмма ВолША [36]. Пусть $f: A \rightarrow K$ - отображение произвольного подмножества пространства $X$ в метризуемый локально компактный ANR. Тогда найдется такое отображение $g: U \rightarrow K$ открытого множсества $U \subset X$, ограничение которого на $A$ гомотопно $f$.

ДокАЗАТЕльство. Выберем на $K$ метрику положительного гомотопического радиуса $r$ и диаметра $d$. Множество $F=\left\{x \in X \mid\right.$ osc $\left._{x} f \geqslant r\right\}$ замкнуто. Пусть $A^{\prime}$ представляет собой разность замькания $A$ и $F$. Доопределим $f$ в точках $x \in A^{\prime} A$, выбирая $f(x)$ из множества $[f](x)$ на основании аксиомы произвольного выбора. При таком доопределении отображения $f$ его колебания не меняются, оставаясь меньше гомотопического радиуса. Поэтому найдется непрерьвное отображение $g^{\prime}: A^{\prime} \rightarrow K$, которое $d / 3$-близко к $f$, и потому ему гомотопно. Множество $A^{\prime}$ замкнуто в $X F$, и потому найдется некоторое открытое в $X F$, а значит, и в $X$, множество $U$, на которое непрерьвно продолжается $g^{\prime}$. Это продолжение и является искомым $g$.

Следуюшая теорема, по сушеству, содержится у Волша [36].

ТЕорема о монотонностИ ЭКСТмерностИ. Если сепарабельное метрическое пространство $X$ әкстенсивно по отношению к комплексу $L$ u $X^{\prime} \subset X$, то и $X^{\prime} \tau L$, если или $X^{\prime}$ открыто, или $L$ метризуем и локально компактен.

ДокАЗАТЕльство. Если $X^{\prime}$ замкнуто, то это очевидно. Если $X^{\prime}$ открыто, то оно является объединением счетного числа замкнутых, и $L$-экстенсивность его вытекает из теоремы счетной суммы. Пусть теперь $X^{\prime}$ - любое подмножество и дано отображение $f: A \rightarrow L$ некоторого его замкнутого подмножества. По лемме Волша найдется такая открытая окрестность $O A$, на которую продолжается отображение, гомотопное отображению $f$. Пусть $A^{\prime}$ является пересечением замыкания $A$ с дополнением к 
$O A$. Тогда разность замькания $A$ и множества $A^{\prime}$ замкнута в открытом множестве $X \backslash A^{\prime}$. В силу монотонности экстмерности по открытым множествам отсюда вытекает возможность продолжить отображение, гомотопное $f$, на множество $X \backslash A^{\prime}$, которое содержит $X^{\prime}$. Что и требовалось.

Джойн. Через $M * N$ обозначается джойн комплексов $M$ и $N$. То есть фактор-пространство произведения $M \times[0,1] \times N$ по отношению $(x, t, y) \simeq\left(x^{\prime}, t, y^{\prime}\right)$, если $t=0$ и $y=y^{\prime}$ или если $t=1$ и $x=x^{\prime}$. Таким образом, нижнее основание джойна $t=0$ гомеоморфно $M$, а верхнее $-N$. Мы в дальнейшем нередко будем отождествлять $M$ и $N$ с его основаниями. Имеются естественные отображения $\pi: M * N \rightarrow[0,1]-$ уровневая проекиия, $\pi_{M}: M * N N \rightarrow M-$ нижняя проекиия и $\pi_{N}: M * N M \rightarrow N$ - верхняя проекиия.

ТЕОРемА УРЫСОНА-ДЫДАКА [24]. Если сепарабельное метрическое пространство $X$ представлено в виде обвединения двух подпространств $X=X_{1} \cup X_{2}$, из которых первое әкстенсивно относительно комплекса $M$, а второе относительно $N$, то $X \tau M * N$.

ДокАЗАТЕЛЬство. Пусть даны замкнутое множество $A \subset X$ и отображение $f: A \rightarrow M * N$. Пусть $X_{1}^{\prime}=X_{1} f^{-1} N$ и $X_{2}^{\prime}=X_{2} f^{-1} M$. Обозначим через $f_{1}$ отображение, непрерьвно продолжающее на $X_{1}^{\prime}$ композицию $\pi_{M} f$, а через $f_{2}-$ продолжающее $\pi_{N} f$ на $X_{2}^{\prime}$. Такие сушествуют в силу монотонности экстмерности. Лемма Волша позволяет считать отображения $f_{i}$ определенными на открытых окрестностях $O_{i} \supset X_{i}^{\prime} \cup A$. Тогда $O_{1} \cup O_{2}=X$ и дополнения до множеств $O_{i}$ являются замкнутыми непересекающимися множествами. Пусть $\varphi: X \rightarrow[0,1]-$ функция Урысона, принимающая значение 0 на дополнении к $O_{2}$ и 1 на дополнении к $O_{1}$, которая на $A$ совпадает с $\pi f$. Тогда искомое продолжение задается формулой $x \rightarrow\left(f_{1}(x), \varphi(x), f_{2}(x)\right)$.

Частичным отображением пространства мы назьваем отображение его замкнутого подмножества. Последовательность частичных отображений $f_{n}$ компакта $X$ в комплекс $L$ называем представительной, если для любого подкомпакта $X^{\prime} \subset X$, на которьй продолжимы все частичные отображения этого семейства, вьполнено условие $X^{\prime} \tau L$.

ЛЕММА О ПРЕДСТАВИТЕЛЬНОЙ ПОСЛЕДОВАТЕЛЬНОСТИ. Для любbx комnaкта $X$ и комплекса L существует представительная последовательность $\left\{f_{n}: X \rightarrow L\right\}$.

ДокАЗАТЕЛЬСтво. Рассмотрим какую-то аддитивную (т.е. замкнутую по конечным объединениям) счетную базу открытых множеств $\left\{U_{i}\right\}$ компакта $X$. Для $i$-го элемента базы выберем последовательность отображений $f_{i j}:\left[U_{i}\right] \rightarrow L$, представляюшую все гомотопические классы отображений из его замыкания в рассматриваемьй комплекс. Тогда совокупность всех $\left\{f_{i j}\right\}$ будет представительной. Действительно, для любого компакта $X^{\prime} \subset X$, любого его частичного отображения $g: X^{\prime \prime} \rightarrow L$ это отображение продолжится на окрестность, а всякая окрестность компакта содержит в себе меньшую, принадлежащую аддитивной базе, поэтому $g$ окажется продолженньм на некоторое $U_{i}$ и, значит, гомотопным некоторому $f_{i j}$, а раз последнее продолжается на $X^{\prime}$, то и $g$ туда продолжается по лемме Борсука. 
Пусть $f: A \rightarrow L$ и $g: B \rightarrow K-$ два частичных отображения компакта $X$. Назовем соединяющим всякое отображение $\varphi: A \cup B \rightarrow L * K$, которое удовлетворяет условиям $f=\pi_{L} \varphi$ и $g=\pi_{K} \varphi$.

ЛЕММА О СОЕДИНЯЮШЕМ ОТОБРАЖЕНИИ. ДЛя любых двух частичных отображений $f: A \rightarrow L$ и $g: B \rightarrow K$ компакта $X$ существует соединяющее отображение, и любье два соединяющих отображсения гомотопны.

ДокАЗАТЕЛЬСтво. Не теряя общности, можем предполагать отображения $f$ и $g$ определенными на открытых окрестностях $O A$ и $O B$ множеств $A$ и $B$. Зафиксируем функцию Урысона $\varphi: X \rightarrow[0,1]$ для пары $A \backslash O B$ и $B \backslash O A$, принимающую на первом значение 0 , а на втором 1 . Тогда соединяющее отображение может быть задано формулой $x \rightarrow(f(x), \varphi(x), g(x))$. Эта формула корректно определяет отображение в джойн, ибо там, где не определено $f(x)$, будет $\varphi(x)=1$, и потому значение отображения определяется уже одним $g(x)$. Аналогичное имеет место в случае, когда не определено $g(x)$. Если имеются два соединяюших отображения $\alpha_{0}$ и $\alpha_{1}$, то гомотопия $\alpha_{t}$ между ними проходит также через соединяюшие отображения, а потому для ее задания достаточно указать уровневую проекцию, которая задается формулой $\pi \alpha_{t}=t \alpha_{1}+(1-t) \alpha_{0}$.

Следующая теорема, доказанная Дранишниковым [37], является экстмерным аналогом теоремы Эйленберга.

ТЕОРЕМА ЭЙЛЕНБЕРГА-ДРАНИШНИКОВА. Если джойн комплексов $K * L$ коәкстенсивен пространству $X$, то любое отображсение $f: X^{\prime} \rightarrow K$ замкнутого в $X$ множества может быть продолжено на дополнение к некоторому замкнутому L-экстенсивному $X^{\prime \prime} \subset X$.

ДокАЗАТЕЛЬство. Пусть $g_{i}: X_{i} \rightarrow L-$ представительная последовательность частичных отображений компакта $X$ в комплекс $L$. Пусть $f * g_{1}: X \rightarrow K * L$-какое-то соединяющее отображение для $f$ и $g_{1}$. Тогда композиция $f_{1}=\pi_{K} f * g_{1}$ определена на $X_{1}=X^{\prime} \cup\left(f * g_{1}\right)^{-1}(K)$, продолжает $f$ и отображение $g_{1}$ продолжается на $X X_{1}^{\prime}$ (а именно, продолжается посредством $\pi_{L} f * g_{1}$ ). Пусть $O X_{1}$ - некоторая замкнутая окрестность множества $X_{1}$, на которую можно продолжить $f_{1}$. Зафиксируем такое продолжение и будем его также обозначать $f_{1}$.

Продолжаем по индукщии строить растущую последовательность замкнутых множеств $X_{i}$ и их замкнутых окрестностей $O X_{i}$ так, чтобы $O X_{i+1} \supset X_{i+1} \supset O X_{i}$, и такую согласованную (т.е. значения отображений совпадают там, где они определены) систему отображений $f_{i}: O X_{i} \rightarrow K$, чтобы все отображения $g_{j}$ при $j \leqslant i$ продолжались на $X X_{i}$.

Очередной шаг индукции подобен первому шагу. Если уже построено $f_{n}: X_{n} \rightarrow K$, то полагаем $X_{n+1}=O X_{n} \cup\left(f * g_{n+1}\right)^{-1}(K)$ и $f_{n+1}=\pi_{K} f * g_{n+1}$ и после доопределяем $f_{n+1}$ на некоторой замкнутой окрестности $O X_{n+1}$.

Теперь согласованная система отображений $f_{i}$ определяет нам некоторое отображение $F$, продолжающее $f$, заданное на объединении окрестностей $\cup O X_{i}$ и непрерьвное на объединении их внутренностей, обозначаемом $U$. Тогда $U \supset \cup X_{i}$, и дополнение к $U$ экстенсивно по отношению к $L$, ибо на него по построению продолжаются все отображения представляющей последовательности. Что и требовалось доказать. 
УТочненИЕ. При тех же предположениях, что имеются в теореме Эйленберга-Дранишникова, моэнно дополнительно добиться, чтобы $X^{\prime \prime}$ не пересекало любого наперед заданного $K$-әкстенсивного сигма-компактного множества $Z$.

ДокАЗАТЕльство. Если $Z=\bigcup Z_{i}$, где $Z_{i}$ компактны, то можно при построении очередного $X_{i}$ к нему добавлять $Z_{i}$, ведь нет препятствий для продолжения $f_{i}$ на $Z_{i}$.

В случае, когда один из комплексов в теореме Эйленберга-Дранишникова является нульмерной сферой, получаем следствие, которое позволяет доказать, что экстраординарная теория, порожденная комплексом, удовлетворяет аксиоме границы.

ТЕОРЕМА О ПЕРЕГОРОДКЕ. Если компакт $X$ әкстенсивен по отношению $\kappa$ надстройке $\Sigma K$ некоторого комплекса, то для любьх двух непересекающихся замкнутых множеств $A, B \subset X$ найдется такое открытое множество $U$, что $A \subset U, B$ лежит в дополнении замыкания множества $U$ и граница $U$ является К-экстенсивной.

Экстмерная теорема о пополнении, доказанная Ольшевским [26], сушественно труднее теоремы Л.А. Тумаркина для обычной размерности и играет в теории экстмерности большую роль.

ТЕОРЕМА ОЛЬШЕВСКОГО. ДЛя любого сепарабельного метрического пространства $X$, әкстенсивного по отношению $к$ счетному комплексу $K$, найдется такое пополнение $\widetilde{X}$, что $\widetilde{X} \tau K$.

ДокАЗАТЕЛЬСтво. Для отображения $f$ через $D(f)$ обозначаем область его определения. Нам придется здесь иметь дело с отображениями, имеюшими различные области определения. В связи с этим уточним, что никакие отображения, имеющие различные области определения, не будут считаться гомотопньми.

Считаем, что $X$ является подпространством гильбертова куба $Q$. Зафиксируем на $Q$ аддитивную счетную базу открытых множеств $\mathscr{B}$. Пару множеств из $\mathscr{B}$ мы будем называть допустимой, если замыкание меншшего множества содержится во внутренности большего.

Для каждого элемента базы зафиксируем по представителю из каждого гомотопического класса отображений этого элемента в $K$. Для каждого такого представителя $f: B \rightarrow K$ зафиксируем некоторую окрестность пространства $X$, на которую можно продолжить отображение $f$, и само продолжение $f^{\prime}$. Так что $D\left(f^{\prime}\right) \supset B$ и $X \subset$ int $D\left(f^{\prime}\right)$. Счетное множество всех таких продолжений обозначим через $K^{\mathscr{B}}$.

Отображение, определенное на некоторой окрестности пространства $X$, будем называть надотображсением пространства $X$.

Пусть дано надотображение $f: O X \rightarrow K$ нашего пространства и две допустимые пары множеств $V \subset U$ и $C \subset D$. Мы будем говорить, что $f$ совместимо с этой парой пар, если $D \cap V \subset O X$. И, наконец, скажем, что отображение $g: C \cap U \rightarrow K$ согласовано с $f$, если его ограничение на $C \cap V$ совпадает с $f$.

Для отображения $f: O X \rightarrow K$ и совместимой с ним пары допустимых пар множеств $V \subset U, C \subset D$ и согласованного отображения $g: C \cap U \rightarrow K$ определим $n е$ рестройку отображения $f$ посредством пятерки $V, U, C, D, g$ как любое отображение $f^{\prime}: O^{\prime} X \rightarrow K$ некоторой окрестности пространства $X$, которое на $C \cap U$ совпадает с $g$, 
а на $(O X \cap V) \cup(O X$ int $D)$ совпадает с $f$. Возможность перестройки доказьвается ввиду замкнутости $(V \cap O X) \cup(O X \operatorname{int} D) \cup(U \cap C)$ в $O X \cup \operatorname{int} D$, которая в свою очередь вытекает из условия совместимости.

Систему надотображений $\mathscr{F}$ пространства $X$ в комплекс $K$ назовем nерестраиваемой, если для любого ее элемента $f: O X \rightarrow K$, любой совместимой с ним пары допустимых пар $V \subset U, C \subset D$ и любого отображения $g: C \cap U \rightarrow K$ найдется отображение $f^{\prime} \in \mathscr{F}$, которое является перестройкой отображения $f$ относительно пятерки $V, U, C, D, g^{\prime}$, где $g^{\prime}: C \cap U \rightarrow K$ - отображение, гомотопное $g$.

Докажем, что всякая счетная система надотображений содержится в счетной перестраиваемой системе надотображений. Действительно, для любой данной счетной системы $\mathscr{F}$ надотображений пространства $X$ множество всех перестроек ее элементов счетно, поэтому по индукции можно построить растушую последовательность $\mathscr{F}=\mathscr{F}_{1}, \mathscr{F}_{2}, \ldots, \mathscr{F}_{n}, \ldots$ счетных систем надотображений так, чтобы $\mathscr{F}_{i+1}$ содержало перестройки всех элементов из $\mathscr{F} i$. Тогда объединение элементов этой последовательности является, очевидно, счетной перестраиваемой системой.

Итак, пусть $\mathscr{F}=\left\{f_{i}: O_{i} X \rightarrow K\right\}$ - некоторая перестраиваемая счетная система надотображений пространства $X$ в комплекс $K$, содержашая $K^{\mathscr{B}}$. Пусть $X^{\prime}=$ $\bigcap_{i=1}^{\infty} O_{i} X$. Тогда $X^{\prime}$ и будет пополнением пространства $X$, экстенсивньм относительно $K$. Докажем это. Пусть дано $f: A \rightarrow K$ - частичное отображение из $X^{\prime}$ в $K$. В силу леммы Волша мы не ограничим общности рассуждений, если будем считать, что $f$ определено на некотором открытом множестве $U$ гильбертова куба. Для построения продолжения этого отображения на $X$ фиксируем две последовательности $U_{i}$ и $A_{i}$ элементов базы $\mathscr{B}$ так, чтобы при любом $i$ пары $U_{i} U_{i+1}$ и $A_{i}, A_{i+1}$ были допустимьми, последовательность $U_{i}$ была возрастающей и имела объединением $U, \mathrm{a}$ последовательность $A_{i}$ была убьвающей и имела пересечением $A$.

Обозначим через $\mathscr{G}$ множество всех надотображений из $X$ в $K$, которые гомотопны отображениям из $\mathscr{F}$.

По индукции строим последовательность $g_{i}$ отображений из $\mathscr{G}$ так, чтобы:

(1) $D\left(g_{i}\right) \supset U_{i} \cap A_{i}$,

(2) $g_{n}(x)=g_{n+1}(x)$, если $x \in X^{\prime} \cap\left(\left(Q\right.\right.$ int $\left.\left.A_{n}\right) \cup U_{n}\right)$,

(3) $g_{n}(x)=f(x)$ для $x \in A_{n} \cap U_{n}$.

В качестве первого отображения $g_{1}$ возьмем любое продолжение отображения $f$, ограниченного на $U_{1}$, гомотопное отображению из $K^{\mathscr{B}}$.

Если отображение $g_{n}$, удовлетворяющее перечисленным условиям, уже построено, то пара пар $U_{n}, U_{n+1}, A_{n+1}, A_{n}$ является совместимой с $g_{n}$ ввиду первого условия. Ограничение отображения $f$ на $U_{n+1} \cap A_{n+1}$ в силу третьего условия согласовано с $g_{n}$, и потому можем определить $g_{n+1}$ как перестройку отображения $g_{n}$ по пятерке $U_{n}, U_{n+1}, A_{n+1}, A_{n},\left.f\right|_{U_{n+1} \cap A_{n+1}}$. Тогда все условия выполняются и индукция продолжается. В результате последовательность $g_{n}$ определяет отображение из $X^{\prime}$ в $K$, являюшееся продолжением отображения $\left.f\right|_{A}$. Что и требовалось доказать.

Следуюшая теорема Дранишникова обрашает теорему Урысона-Дыдака и усиливает теорему Эйленберга-Дранишникова.

ТЕОРема УРЫСОНА-ДРАНИШНИКОВА. Если компакт $X$ әкстенсивен по отношению $к$ джойну комплексов $K * L$, то существуют такие $G_{\delta}$-множества $G_{1} u G_{2}$, что $X=G_{1} \cup G_{2} u G_{1} \tau K, G_{2} \tau L$. 
ДокАЗАТЕльство. Пусть $\left\{f_{i}\right\}$ - представительная последовательность частичных отображений из $X$ в $L$. Для каждого такого отображения в силу теоремы Эйленберга-Дранишникова найдется замкнутое $K$-экстенсивное множество $X_{i}$, на дополнение к которому продолжается отображение $f_{i}$. Тогда объединение $X_{i}$ является $K$-экстенсивным $F_{\sigma}$-множеством, а на дополнение к этому объединению продолжаются все отображения $g_{i}$, и потому все компактные (а значит, и $F_{\sigma^{-}}$) подмножества в нем будут $L$-экстенсивны. Теперь в силу теоремы Ольшевского найдется $G_{\delta}$-множество $G_{1}$, содержашее объединение $\cup X_{i}$ и также $K$-экстенсивное. Тогда дополнение к нему согласно сделанному вьше замечанию будет $L$-экстенсивно. Применяя к этому дополнению теорему Ольшевского, получаем искомое $G_{2}$.

УТОчненИЕ. При условиях теоремы о разложении можно дополнительно добиться, чтобы $G_{1}$ содержало любое наперед заданное сигма-компактное $K$-экстенсивное множество $Z$.

ДокАЗАТЕльство. Все $f_{i}$ можно считать содержашими $Z$ в силу уточнения к теореме Эйленберга-Дранишникова.

\section{$\S$ 5. Критерий экстенсивности}

Следуюшая теорема Дранишникова, представляюшая собой половину сформулированного во введении критерия экстенсивности, играет в теории размерности роль аналогичную той, которую играет в теории гомотопий теорема Гуревича об изоморфизме первых нетривиальных групп гомологий и гомотопий. Абелевьли мы, следуя М.М. Постникову [32], назьваем гомотопически простые пространства, т.е. пространства с тривиальным действием фундаментальной групшы на гомотопиях.

ТеоРема ГУРеВИЧА-ДРАНИШНИКОВА. Для абелева комплекса $K$ полигруппа гомотопий $\pi_{*} K$ изофакторна полигруппе гомологий $H_{*} K$, m.e. $\sigma_{p} \pi_{*} K=\sigma_{p} H_{*} K$ для любого $p \geqslant 0$.

Для доказательстванам потребуется как теорема Гуревича по модулю класса Серра абелевых груп, которую мы будем назьвать теоремой Гуревича-Серра, так и теория локализации и пополнения Сулливана [38].

Применение теоремы Гуревича-Серра по модулю класса Серра групा $G$, для которых $G \otimes \mathbb{Z}_{(p)}=0$, позволяет доказать совпадение всех гомотопических и гомологических $p$-факторов абелева комплекса, за исключением метарациональных. Для метарационального случая приходится применять теорию Сулливана локализации и пополнения гомотопического типа. Из этой теории получается заодно и доказательство в дорациональном случае. Поэтому перейдем к изложению результатов Сулливана.

Напомним понятие локализации гомотопического типа. Будем считать далее, что $\mathbb{Z}_{(0)}=\mathbb{Q}$, чтобы не говорить отдельно о локализации в нуле. $p$-локализующим гомоморфизмом групшы $G$ назьвается ее гомоморфизм в $G \otimes \mathbb{Z}_{(p)}$, переводяший $g$ в $g \otimes 1$. Групша назьвается $p$-локальной, если этот гомоморфизм является изоморфизмом.

ТЕОРема СУЛЛИВАНА О ЛОКАЛИЗАЦИИ. Для любых абелева комплекса $K u$ простого $p \geqslant 0$ существуют комплекс $K_{(p)}$, называемый $p$-локализацией комплекса $K$, и такое отображение $l_{p}: K \rightarrow K_{(p)}$ (локализующее отображсение), для 
которого все индуцированные гомоморфизмы всех гомотопических и гомологических групп положительных размерностей являются р-локализующими.

Этой теоремы хватает для доказательства теоремы Гуревича-Дранишникова для дорациональных факторов. Так, для доказательства совпадения бокштейновских 0 -факторов $\pi_{*} K$ и $H_{*} K$ рассмотрим 0-локализацию $l_{0}: K \rightarrow K_{(0)}$. Согласно теореме Гуревича первая нетривиальная гомотопическая группа у $K_{(p)}$ встречается в той же размерности $n$, что и гомологическая. То есть $\pi_{k} \otimes \mathbb{Q}=H_{k} \otimes \mathbb{Q}=0$ при $k<n$ и $\pi_{n} \otimes \mathbb{Q}=H_{n} \otimes \mathbb{Q} \neq 0$. Но это означает, что $\sigma_{0} H_{k}=\sigma_{0} \pi_{k}=0$ при $k<n$ и $\sigma_{0} H_{k}=\sigma_{0} \pi_{k}=\mathbb{Q}$ при $k=n$, что и означает равенство $\sigma_{0} H_{*}=\sigma_{0} \pi_{*}$. Аналогично доказывается совпадение дорациональных $p$-факторов. Потому что для групшы $G$ наличие ненулевого $p$-фактора равносильно нетривиальности произведения $G \otimes \mathbb{Z}_{(p)}$, и для любой периодической групшы $G \otimes \mathbb{Z}_{(p)}=G_{p}$ имеет такой же $p$-фактор, что и $G$. В случае, когда первьй $p$-фактор Бокштейна встречается в той же размерности, что и рациональный, ибо $\sigma_{p} G=\sigma_{p}\left(G \otimes \mathbb{Z}_{(p)}\right)$ (лемма о факторе локализации).

Для выявления метарациональных факторов нам потребуется следующая теорема, уточняющая теорему Сулливана о том, что локальность всех гомологических групш абелева комплекса равносильна локальности всех его гомотопических групп.

ТЕОРЕМА ГУРеВИЧА-СуЛЛИВАНА. Для абелева комплекса $K$ первая размерность $n$, в которой встречается не р-локальная гомотопическая группа, совпадает с первой размерностью $m$, в которой встречается первая не р-локальная гомологическая группа.

ДоКАЗАТЕльство. Предположим противное. Пусть, например, $n<m$. Возьмем $m$-мерный остов комплекса $K$ и добавим к нему $(m+1)$-мерные клетки так, чтобы убить $m$-мерные гомологии. Полученное пространство $K^{\prime}$ имеет $p$-локальными все гомологии, ибо его гомологии совпадают с гомологиями $K$ в размерностях $<m$ и равны нулю в остальных размерностях. А гомотопии этого пространства совпадают с гомотопиями комплекса $K$ в размерностях меньше $m$ и, в частности, в размерности $n$ и не являются $p$-локальными вопреки теореме Сулливана о равносильности локальности. Если $n>m$, то мы заклеиваем все гомотопии комплекса $K$ начиная с размерности $n$ и получаем аналогичное противоречие.

Применение теоремы Гуревича-Сулливана позволяет доказать, что размерности появления нетривиальных $p$-факторов у гомотопических и гомологических групп комплекса $K$ совпадают даже в метарациональном случае, но не позволяет определить тип этих факторов. А именно, рассмотрим $p$-локализованный комплекс $K_{(p)}$. Пусть $q$ - простое число, отличное от $p$, тогда размерность появления первого нетривиального $p$-фактора совпадает с размерностью первой не $q$-локальной группы (ведь $\mathbb{Q}$ локальна для любого простого), и поэтому теорема Гуревича-Сулливана позволяет утверждать совпадение размерностей гомологического и гомотопического $p$-факторов. Для определения их типа потребуется следующая теорема Сулливана о пополнении. $p$-пополнение группы $G$ - это обратньй предел из факторгрупп $G / p^{n} G$; обозначение: $G_{[p]}$. Важно, что пополнение рациональных чисел, как и любой $p$-делимой группы, тривиально. В частности, тривиально оно и для $\mathbb{Q}_{p}$. Для $\mathbb{Z}_{(p)}$ пополнением является кольцо целых $p$-адических чисел, которое ему факторно эквивалентно, ибо кручения не имеет и делится на все простые числа кроме $p$. 
ЛЕММА о ФАКТорАХ ПОПОЛнЕНИЯ. $\sigma_{q} G_{[p]}=0$ npu $q \neq p, \sigma_{p} G_{[p]}=\sigma_{p} G$, ecли $\sigma_{p} G$ равняется $\mathbb{Z}_{(p)}$ или $\mathbb{Z}_{p}$, иначе $\sigma_{p} G_{[p]}=0$.

ДокАЗАТЕЛЬСТВО. Всякий элемент $g \in G$, не делящийся на $p^{n}$ для какого-то $n$, переходит при пополняюшем гомоморфизме не в нуль, ибо не нули его образыв $G / p^{m} G$ при $m>n$. Порядок элемента при переходе к пополнению не меняется. Поэтому если в $G$ наличествовали элементы бесконечного порядка, не деляшиеся на $p$, то таковые будут и в пополнении. То есть, если $\sigma_{p} G=\mathbb{Z}_{(p)}$, то и $\sigma_{p} G_{[p]}=\mathbb{Z}_{(p)}$. Если же таковых элементов бесконечного порядка не было, а были конечного, то есть, в случае $\sigma_{p} G=\mathbb{Z}_{p}$, то тоже будет для $\sigma_{p} G_{[p]}$. При любом $n$ групп $G / p^{n} G$ является $p$-группой и однозначно делима на $q$, поэтому и предел делим на $q$. В то же время в пределе нет элементов порядка $q$. Этого хватает для доказательства равенства $\sigma_{q} G_{[p]}=0$.

ТЕОРЕМА СУЛЛИВАНА О ПОПОЛНЕНИИ. Для любого простого $p$ и любого абелева комплекса $K$ существует такой комплекс $K_{[p]}$, называемый р-пополнением комплекса $K$, все гомотопические и гомологические группы которого являются р-пополнениями соответствующих групп комплекса $K$.

Итак, рассмотрим $p$-пополнение нашего комплекса $K$; применим к нему теорему Гуревича. Ввиду доказанной леммы о факторах пополнения мы получим такой результат. Первые $p$-факторы гомотопий и гомологий, отличные от $\mathbb{Q}_{p}$, совпадают как по типу, так и по месту появления. Эта информашия, дополненная ранее добытой информацией о совпадении первых мест появления гомологических $p$-факторов, позволяет восстановить как тип, так и место появления первых гомотопических и гомологических факторов и убедиться в их совпадении.

А именно, определение типа первого $p$-фактора происходит по следуюшему алгоритму. Если места его появления в группах комплекса $K$ и его $p$-пополнения не совпадают, то этот фактор есть $\mathbb{Q}_{p}$, иначе он совпадает с первым $p$-фактором пополнения.

Итак, теорема Гуревича-Дранишникова полностью доказана. Доказанная вьше теорема несколько точнее доказанной Дранишниковым в [21]. То, что доказано там, в нашей терминологии соответствует совпадению высотностей гомотопической и гомологической полигрупп, т.е. их унифищированных $p$-факторов Бокштейна. Отметим такое следствие этой теоремы:

О ГОМОЛОГИЯХ КОМПЛЕКСОВ ЭЙЛЕНБЕРГА-МАКЛЕЙНА. Группа $G$ факторно доминирует любую группу гомологий $H_{m}(K(G, n))$ комплекса Эйленберга-Маклейна.

Действительно, все нетривиальные факторы Бокштейна в группах гомотопий у комплекса Эйленберга-Маклейна появляются одновременно, ибо у него единственная нетривиальная группа гомотопий. Значит, и для гомологий это так. Что как раз и означает то, что утверждается.

ГОМОЛОГИЧЕСКИЙ КРИТЕРИЙ ЭКСТЕНСИВНОСТИ. Для әкстенсивности конечномерного компакта $X \kappa$ связному абелеву комплексу $K$ необходимо и достаточно, чтобы при любом $n>0$ были выполненьи неравенства $\operatorname{dim}_{H_{n}(K)} X \leqslant n$.

Сформулированное условие равносильно, как мы знаем, совместимости полигруш $\mathscr{H}^{*}(X)$ и $H_{*}(K)$, а в силу теоремы Гуревича-Дранишникова полигрупшу гомологий можно заменить здесь на полигруппу гомотопий. Совместимость же полигрупп 
$\mathscr{H}^{*}(X)$ и $\pi_{*}(K)$ расшифровьвается как цепочка неравенств $\operatorname{dim}_{\pi_{n} K} X \leqslant n$ для любого $n$. Поэтому достаточность гомологического условия экстенсивности следует из гомотопического.

ГОМОТОПИЧЕСКОЕ УСЛОВИЕ ЭКСТЕНСИВНОСТИ. Если для конечномерного компакта $X$ при любом натуральном $n$ выполнены неравенства $\operatorname{dim}_{\pi_{n}} X \leqslant n$, mo $X \tau K$.

ДоказАТЕЛЬСтво. Пусть дано отображение $f: A \rightarrow K$ какого-то замкнутого множества $A \subset X$. Рассмотрим систему Постникова комплекса $K$ и будем по индукции строить продолжения отображений $i_{n} f$ (где $i_{n}: K \rightarrow K^{(n)}$ - канонические отображения) в комплексы $K^{(n)}$ - элементы этой системы. Для $n=1$ имеем: $K^{(1)}$ имеет тип комплекса Эйленберга-Маклейна $K\left(\pi_{1}(K), 1\right)$, и продолжение возможно в силу того, что $\operatorname{dim}_{\pi_{1}} X \leqslant 1$. Если уже построено отображение $f_{n}: X \rightarrow K^{(n)}$, продолжаюшее $i_{n} f$, то рассмотрим расслоение Серра $p_{n}: K^{(n+1)} \rightarrow K^{(n)}$. У нас уже имеется отображение $i_{n+1} f: A \rightarrow K^{(n+1)}$, накрьвающее отображение $i_{n} f: A \rightarrow K^{(n)}$ так, что $p_{n} i_{n+1} f=i_{n} f$. Попробуем продолжить это поднятие до отображения $f_{n+1}$ такого, что $p_{n} f_{n+1}=f_{n}$. Слоем отображения $p_{n}$ служит комплекс Эйленберга-Маклейна $K\left(\pi_{n+1} K, n+1\right)$. Единственное возникаюшее при этом препятствие лежит в когомологиях $H^{n+2}\left(X, A, \pi_{n+1} K\right)$. Но эта группа нулевая ввиду условия $\operatorname{dim}_{\pi_{n+1} K} X \leqslant$ $n+1$. Таким образом, отображение $f_{n+1}$ можно построить. Так, построим отображение $f_{N}$ для $N>\operatorname{dim} X$. Но отображение $i_{N}$ является гомотопической эквивалентностью до размерности $N$, и потому найдется отображение $F: X \rightarrow K$, ограничение которого на $A$ совпадает с $f$, а композиция $i_{N} F$ гомотопна $F$. Это $F$ и есть то, что надо было построить.

Доказательство необходимости. Перейдем к доказательству необходимости гомологического условия в критерии экстенсивности. Для пространства $X$ через $\mathrm{SP}^{n} X$ обозначается его $n$-я симметрическая степень, т.е. факторпространство его $n$-й степени по действию групшы перестановок координат. Элементы $\mathrm{SP}^{n}$ - такие наборы точек пространства $X$ с кратностями, что сумма кратностей равна $n$. Если в $X$ отмечена точка, то $\mathrm{SP}^{n}$ можно вложить в $\mathrm{SP}^{n+1}$ отображением, которое увеличивает на единицу кратность отмеченной точки. Возникающий при этом прямой предел из $\mathrm{SP}^{n}$ назьвается бесконечной симметрической степенью $\mathrm{SP}^{\infty} X$ пространства $X$.

Следующее рассуждение принадлежит Дранишникову [21].

ЛЕММА О СИММЕТРИЧЕСКОЙ СТЕПЕНИ. Если компакт $X$ әкстенсивен по отношению $к$ комплексу $K$, то $X \tau \mathrm{SP}^{\infty} K$.

ДокАЗАтельство. Пусть $f: A \rightarrow \mathrm{SP}^{\infty} K$ - какое-то частичное отображение компакта $X$. Тогда образ $f(A)$, будучи компактом, лежит в некотором $\mathrm{SP}^{n} K$. Зафиксируем вложение $K$ в гильбертов куб $Q$. Воспользовавшись тем фактом, что $\mathrm{SP}^{n} Q$ является абсолютным ретрактом, продолжим отображение $f$ до отображения $F: X \rightarrow \operatorname{SP}^{n} Q$. Пусть $G(F)=\{(x, y) \in X \times Q \mid y \in F(x)\}$ (здесь принадлежность точки из $Q$ к элементу из $\operatorname{SP}^{n} Q$ трактуется как ее ненулевая кратность). Проекция произведения конечнократно отображает $G(F)$ на $X$ и в силу леммы о конечнократных отображениях из $\S 7$ получаем, что $G(F) \tau K$. Обозначим через $G(A)$ пересечение $G(F)$ и $A \times Q$. Тогда $G(A)$ при проектировании на $Q$ попадает в $K$. Продолжим это 
отображение до отображения $\Phi: G(F) \rightarrow K$. Через $\mathrm{SP}^{n} \Phi$ обозначим индуцированное им отображение симметрических степеней. Тогда композиция $\mathrm{SP}^{n} \Phi F$ является искомьм продолжением.

Следующая неэлементарная теорема представляет основной аргумент в доказательстве критерия экстенсивности в части необходимости.

ТЕОРема ДОЛЬДА-ТОма [39]. Для любого комплекса $K$ его бесконечная симметрическая степень $\mathrm{SP}^{\infty}$ K имеет слабый гомотопический тип произведения комплексов Эйленберга-Маклейна $\prod K\left(H_{n}(K), n\right)$.

Пусть компакт $X$ экстенсивен к комплексу $K$. Тогда в силу леммы о симметрической степени $X \tau \mathrm{SP}^{\infty} K$. В силу теоремы Дольда-Тома получаем, что $X \tau \prod K\left(H_{n}(K), n\right)$. Откуда получаем, что $X \tau K\left(H_{n}(K), n\right)$ при любом $n$. Следовательно, $\operatorname{dim}_{H_{n}(K)} X \leqslant n$ для любого $n$. Что и требовалось доказать.

\section{§ 6. Размерность и связность}

Кюннетовское представление связностной алгебры. Комплекс по умолчанию означает связный пунктированный, счетный клеточный комплекс с топологией Уайтхеда. Гомологии - приведеннье гомологии. Через $K \vee L$ обозначается букет комплексов, а через $K \wedge L$ - приведенное произведение (smash-product), т.е. $K \wedge L=K \times L / K \vee L$.

Полигруппа целочисленных сингулярных приведенных гомологий комплекса $K$ обозначается $H_{*}(K)$. Она является нижней полигруппой для связного комплекса. Обозначим через $\operatorname{Cin} K$ триплетную последовательность Dep $H_{*}(K)$ и назовем ее связностным кодом комплекса $K$.

Теорема. Отображсение, сопоставляющее связному абелеву комплексу полигруппу его приведенных гомологий, индуцирует изоморфизм межжду связностной алгеброй и нижней алгеброй Кюннета.

ДоКАЗАТЕЛЬСТво основано на трех соображениях:

(1) $H_{*}(K \wedge L)=H_{*} K\langle\times\rangle H_{*} L$;

(2) $\operatorname{cin} K=\operatorname{dep} H_{*} K$;

(3) для всякой нижней полигрупшы $\mathscr{G}$ глубины больше единищы найдется такой односвязный $K$, что $H_{*} K=\mathscr{G}$.

Первое утверждение представляет собой формулу Кюннета для гомологий, а второе - определение. В качестве $K$ для третьего утверждения годится букет комплексов Мура $M\left(G_{k}, k\right)$.

Пусть DEP $H_{*} K \neq \operatorname{DEP} H_{*} L$, тогда Dep $H_{*} K \neq \operatorname{Dep} H_{*} L$. B силу теоремы о тестировании найдется такая кодировка $C$, что $\left\langle\left\langle\operatorname{Dep} H_{*} K\langle+\rangle C\right\rangle\right\rangle\left\langle\left\langle\operatorname{Dep} H_{*} L\langle+\rangle C\right\rangle\right\rangle$. Причем $C$ можно считать имеюшей связность больше единицы, просто увеличив ее на 1 , если необходимо. В силу теоремы кодирования полигрупп $C=\operatorname{Dep} \mathscr{G}$ для некоторой полигруппы $G$. Пусть $M$ - такой комплекс, что $H_{*} M=\mathscr{G}$. Тогда $\operatorname{cin} M \wedge K \neq$ $\operatorname{cin} M \wedge L$, и, следовательно, $\mathrm{CIN} K \neq \mathrm{CIN} L$.

Обратно, пусть $\mathrm{CIN} K \neq \mathrm{CIN} L$. Тогда $\operatorname{cin} M \wedge K \neq \operatorname{cin} M \wedge L$ для некоторого $M$. Откуда $\operatorname{dep}\left(H_{*} M\langle\times\rangle H_{*} K\right) \neq \operatorname{dep}\left(H_{*} M\langle\times\rangle H_{*} L\right)$, и, следовательно, DEP $H_{*} K \neq$ $\mathrm{DEP} H_{*} L$. 
Изотонность отображения $H_{*}$ вытекает из формулы $H_{*}(K \wedge L)=H_{*} K \oplus H_{*}(L)$. Если комплекс $L$ односвязен, то произведение $L \times K\left(G_{1}, 1\right)$ абелево и имеет гомотопии $G_{1}^{(1)} \oplus \pi_{*}(L)$. Ho Alt $\pi_{*}(L)=\operatorname{Alt} H_{*}(L)$ в силу теоремы Гуревича-Дранишникова. Поэтому $\operatorname{Alt}\left(G_{1}^{(1)} \oplus \pi_{*}(L)\right)=\operatorname{Alt}\left(G_{1}^{(1)} \oplus H_{*}(L)\right)$. А так как $H_{*}(L)$ по доказанному вьше может представлять любую полигруппу глубины $>1$, то $G_{1}^{(1)} \oplus H_{*}(L)$ может представлять любую нижнюю полигруппу. Таким образом, установлена сюръективность гомологий.

Итак, гомологии индуцируют биективное изотонное соответствие между $\mathfrak{K}_{*}$ и $\mathfrak{C} \mathfrak{N}$, сохраняющее операцию. Теорема доказана.

Кодирование размерности. Когомологический размерностньй тип компакта $X$ кодируется высотным типом его тотальных когомологий, который по теореме о триплетном представлении представляется триплетной последовательностью, обозначаемой $\operatorname{Dim} X$ и называемой размернымм кодом компакта $X$.

Прежде всего, отметим, что для непустого конечномерного компакта полигруппа его тотальных когомологий является верхней полигруппой. Если компакт нульмерен, то никаких когомологий в положительных размерностях у него нет и его полигрупа тотальных когомологий тривиальна. Для общего случая нужное нам утверждение вытекает из следующей леммы.

ЛЕММА ОБ ОДНОМЕРНЫХ КОГОМОЛОГИЯХ. Для любого компакта $X$ nоложительной размерности и любого замкнутого $A \subset X$ группа $H^{1}(X, A)$ факторно эквивалентна $\mathbb{Z}$ и не имеет кручения.

ДоказАТельство. Во-первых, $H^{1}(X, A)$ не имеет кручения. Действительно, пусть $z \in H^{1}(X, A)$ имеет порядок $n$. Пусть $f: X \rightarrow S^{1}$ - отображение, представляюшее $z$ (для которого $f(A)$ одноточечно). Пусть $g: S^{1} \rightarrow S^{1}$ - возведение в степень $n$. Тогда по предположению композиция $g f$ гомотопна нулю гомотопией, фиксированной на $A$. Но $g$ - накрытие и гомотопию можно поднять. Что и даст стягивание в точку для $f$.

Итак, $H^{1}(X, A)$ не имеет кручения. Обозначим через $C(A)$ конус над $A$ и будем считать, что $C(A) \cup X=A$ является основанием этого конуса. Напишем отрезок точной последовательности пары $C(A) \cup X, X$, заменяя $H^{1}(C(A) \cup X, X)$ на эквивалентное ему $H^{1}(\Sigma A)=H^{0}(A)$ и $H^{1}(C(A) \cup X)$ на $H^{1}(X, A)$ :

$$
H^{0}(A) \rightarrow H^{1}(X, A) \rightarrow H^{1} X \rightarrow 0
$$

Так как группа $H^{0}(A)$ свободна, а $H^{1}(X)$ не имеет кручения при тензорном умножении на любую групу $G$, точность последовательности сохранится, и мы получаем, что нетривиальность $G \otimes H^{0}(A)$ влечет нетривиальность $G \otimes H^{1}(X, A)$. То есть $H^{1}(X, A)$ тензорно эквивалентна $\mathbb{Z}$ и, не имея кручения, будет и факторно эквивалентна $\mathbb{Z}$.

КОДИРОВАННЫЙ КРИТЕРИЙ ЭКСТЕНСИВНОСТИ. Для любого конечномерного компакта $X$ и абелева комплекса $K$ условие $X \tau K$ равносильно неравенству $\operatorname{Dim} X \leqslant \operatorname{Cin} K$. 
ДоказАТЕльСтво. $\operatorname{Dim} X \leqslant \operatorname{Cin} K$, как мы знаем из теоремы о кодировании полигрупп, равносильно совместимости $\mathscr{H}^{*}(X) \tau H_{*}(K)$. А это условие расшифровьвается, в силу высотного критерия совместимости, как совокупность неравенств alt $_{H_{n}(K)} \mathscr{H}^{*}(X) \leqslant n$, которая, в свою очередь, равносильна цепочке $\operatorname{dim}_{H_{n}(K)} X \leqslant$ $n$, что равносильно экстенсивности $X \tau K$ согласно критерию Дранишникова.

\section{Теорема реализации}

Минимальные компакты. Первые компакты с непостоянным размерностным кодом - знаменитые понтрягинские поверхности [6] - имели коды типа $D(q)=1$ при $q \neq p$ и $D(p)=2^{-}$. Их $n$-мерными аналогами естественно считать компакты $\left(n^{-}, p\right)$-минимального размерностного кода $(n>1, p>0)$. Поэтому мы будем называть их компактами Понтрягина.

Следуюший тип кода был реализован В.Г. Болтянским [8]: $D(q)=1$ при $q \neq p$ и $D(p)=1^{+}$. Компакты, размерностный код которых $\left(n^{+}, p\right)$-минимален $(n>0, p>0)$, мы будем называть компактами Болтянского.

Ю. Кодама [12] построил компактыс кодом $D(q)=1^{+}$при $q \neq p, 0$ и $D(p)=D(0)=$ 2 . Компакты $(n, p)$-минимального размерностного кода $(n=|n|>1, p \geqslant 0)$ будем называть компактами Кодама. Причем компакты, реализуюшие $(n, 0)$-минимальный код, называем рачиональным.м компактами Кодама.

Простое число, фигурируюшее в определениях этих компактов, назьвается их факторностью.

Перечисленными типами компактов исчерпьваются все минимальные компакты. Кузьминов называл такие компакты основнылми. Он доказал [14], что через суммы этих компактов можно реализовать компакты любой кодировки. Все минимальные компакты были построены А. Н. Дранишниковым [15].

Компакт $X$ назьвается размерно-однородным, если $\operatorname{Dim} X^{\prime}=\operatorname{Dim} X$ для любого подкомпакта с непустой внутренностью.

Следуюшее предложение уточняет теорему Дранишникова о реализации размерностных типов в $\mathbb{R}^{n}$. Другая теорема о промежуточных экстмерностях содержится в статье $[40]$.

ТЕОРЕМА О ПРОМЕЖУТОЧНОЙ РАЗМЕРНОСТИ. Размерно-однородный компакт $X$ c $\langle\langle\operatorname{Dim} X\rangle \geqslant 2$ содержит подкомпакт $X(C)$ любого размерностного кода $C$, мажсорно подчиненного $\operatorname{Dim} X-1$.

ДокАЗАТЕльство. Во-первых, заметим, что условие $\langle\langle\operatorname{Dim} X\rangle \geqslant 2$ влечет двухсторонность вычитания $\operatorname{Dim} X-1$, в силу критерия двухсторонности из $\S 3$.

Положим $D=(\operatorname{Dim} X-1)\langle-\rangle C$. Пусть $N, M-$ комплексы связностей $\operatorname{Cin} N=D$ и $\operatorname{Cin} M=C$. Поскольку $D\langle+\rangle C+1 \geqslant \operatorname{Dim} X$ в силу критерия экстенсивности получаем $X \tau M * N$. В силу теоремы о разложении имеем $X=Y \cup Z$, где $Y \tau N, Z \tau M$ и $Y=\bigcup Y_{i}$, где все $Y_{i}-$ компакты. Пусть $B=\sup \operatorname{Dim} Y_{i}$. Тогда $B=C$. Действительно, мы знаем, что $X_{i} \tau N$, и потому в силу критерия экстенсивности имеем неравенство $\operatorname{Dim} X_{i} \leqslant C$ при любом $i$. Откуда $B \leqslant D$. Если же предположить, что $B<D$, то в силу экстмерной теоремы счетной суммы получим $Y \tau K$ для абелева комплекса $K$ связности $\operatorname{Cin} K=B$. Но тогда в силу теоремы Урысона-Дыдакаполучим $X \tau K * M$, и в силу критерия экстенсивности $\operatorname{Dim} X \leqslant B\langle+\rangle D+1$. Откуда $B\langle+\rangle D \geqslant \operatorname{Dim} X-1$, 
что противоречит минимальности разложения $\operatorname{Dim} X-1\langle=\rangle C\langle+\rangle D$, вытекающего из условия $C\langle\leqslant \operatorname{Dim} X-1$.

Далее, заметим, что ввиду размерной однородности $X$ то же рассуждение, примененное к $X^{\prime}$ с непустой внутренностью, показьвает, что $\sup \operatorname{Dim}\left(X^{\prime} \cap Y_{i}\right)=C$. Фиксируем точку $x \in X$ и компакт $X(C)$ определяем как объединение последовательности компактов, индексированных простыми числами $X_{p}$, где $X_{p}$ лежит в пересечениии $Y$ с $1 / i$-окрестностью точки $x$ и $\operatorname{Dim}_{p} X_{p}=C(p)$. При этом можем считать все $X_{p}$ содержашими $x$. Тогда их объединение компактно и имеет нужную размерность.

В силу доказанной теоремы и критерия подчинения из $\S 3$ получаем:

РЕАЛИЗАЦИОННАЯ ТЕОРЕМА ДРАНИШНИКОВА. Всякая ограниченная кодировка $N$ является размерностным кодом некоторого компакта $X$, лежащего в $\mathbb{R}^{n}$, если $\|N\|<n-1$.

\section{Компакт без промежуточной размерности}

Проанализируем теперь сушественность требования подчиненности в теореме о промежуточных размерностях.

Объединение $n$-мерного рационального компакта Кодама $K_{0}^{n}$ с $n$-мерным компактом Понтрягина $P_{p}^{n}$ факторности $p$ ни при каком $n$ не содержит двумерного компакта компакта Кодама $K_{p}^{2}$. Действительно, предположим, что $K_{p}^{2} \subset K_{0}^{n} \cup P_{p}^{n}$. Тогда $\operatorname{Dim}_{p}\left(K_{p}^{2} \cap P_{p}^{n}\right) \leqslant 2^{-}$, ибо рациональная размерность этого пересечения не превосходит единицы. И $\operatorname{Dim}_{p}\left(K_{p}^{2} \cap K_{0}^{n}\right) \leqslant 1$, ибо единична размерность $\operatorname{dim}_{p} K_{0}^{n}$. В результате получаем, что $\operatorname{Dim}_{p} K_{p}^{2} \leqslant 2^{-}$, а она должна равняться двум.

Правда, построенный пример не является размерно-однородным, но на его основе можно получить и размерно-однородный пример. Будем говорить, что компакт $Z$ является смесью компактов $Y$ и $X$, если любая его неодноточечная компонента связности гомеоморфна или какой-то компоненте связности компакта $X$, или какой-то компоненте связности компакта $Y$.

Мы построим ниже размерно-однородную смесь для любых компактов. Доказательство того факта, что двумерный $p$-факторный компакт Кодама не содержится также в рационально-понтрягинской смеси, опирается на теорему о размерности компонент и использует те же аргументы, которые были приведены выше при доказательстве того, что он не содержится в объединении понтрягинского компакта и рационального компакта Кодама.

ТЕОРема о СмЕШИВАНИИ. Для любых двух компактов $X, Y$ существует размерно-однородньй компакт $Z$ размерности $\operatorname{Dim} Z=\sup \{\operatorname{Dim} X, \operatorname{Dim} Y\}$, являющийся их смесью.

Доказательство основано на следующей лемме.

ЛЕмма о $\sigma$-РАСШИРЕНИИ. Для любого компакта $X$ и любого компакта $Y$ существует такой компакт $Z \supset X$ (его $Y^{\sigma}$-расширение), что $Z \backslash X$ всюду плотно в $Z$ и является обгединением последовательности непересекающихся компактов, гомеоморфных $Y$, с диаметрами, стремящимися к нулю, и существует ретракиия $r: Z \rightarrow X$. 
ДокАЗАТЕльство. По теореме Урысона мы можем считать $X$ лежашим в гильбертовом кубе $Q$ в качестве нигде не плотного подмножества. Выберем в $Q$ последовательность точек $y_{n}$, не принадлежаших $X$, множество частичных пределов которой совпадает с $X$. У каждой точки последовательности выберем столь малую окрестность $O y_{n}$, чтобы эти окрестности не пересекались ни с $X$, ни между собой, а диаметры их стремились к нулю. Теперь, выбрав в каждой окрестности компакт $Y_{n}$, гомеоморфньй $Y$, что возможно в силу той же теоремы Урысона, в качестве искомого $Z$ можем взять объединение $X$ и последовательности $Y_{n}$. Ретракцию $r$ можно определить, отображая каждое $Y_{n}$ в одну (произвольно выбранную) из ближайших к $y_{n}$ точек компакта $X$.

ДОКАЗАТЕЛЬСТВО ТЕОРЕМЫ О СМЕШИВАНИИ. По ИНдуКцИИ строится Последовательность компактов $Z_{n}$ и ретракций $r_{n}: Z_{n} \rightarrow Z_{n-1}$. Полагаем $Z_{0}=X$, в качестве $Z_{1}$ берем $Y^{\sigma}$-расширение компакта $X$ и в качестве $r_{1}$ - ретракцию этого расширения на $X$. При нечетном $n$ определяем $Z_{n}$ как $Y^{\sigma}$-расширение, а при четном - как $X^{\sigma}$-расширение компакта $Z_{n-1}$, а в качестве $r_{n}$ берем ретракцию расширения. Искомьй компакт $Z$ получается теперь как обратный предел построенной последовательности. Доказательство того, что $Z$ является смесью компактов $X$ и $Y$, основано на следуюшем свойстве ретракции $\sigma$-расширения $r: Z \rightarrow X$ : для любого континуума $C \subset Z$ либо $r(C)$ одноточечно, либо $C \subset X$ и ограничение $r$ на $C$ тождественно.

Есть два случая, когда триплет с малой основой не подчиняется триплету с большой основой. Первьй случай - когда большой сингулярен, а малый регулярен, и второй - ког да большой имеет отрицательную сигнатуру, в то время как малый положительную.

Первому случаю соответствует разобранньй нами вопрос о компактах Кодама. Второй касается нахождения компактов Болтянского в компактах Понтрягина. В связи с последним интересно было бы выяснить следующее:

ПРОБЛЕМА ПРОМЕЖУТОЧНОЙ РАЗМЕРНОСТИ. Существует ли компакт Понтрягина размерности 3 , не содержащий никакой поверхности Болтянского?

\section{Теорема кодирования размерности-связности}

МУЛЬТИПЛИКАТИВНОСТЬ ТОТАЛЬНЫХ КОГОМОЛОГИЙ. Для любой әрупnы $G u$ любых компактов $X, Y$ полигруппа $\mathscr{H}^{*}(X \times Y)$ почленно изофакторна верхнему произведению Кюннета $\mathscr{H}^{*}(X)[\times] \mathscr{H}^{*}(Y)$.

ДокАЗАТЕльСТво. Обозначим через $\mathscr{H}^{* \prime}(X)$ и $\mathscr{H}^{* \prime}(Y)$ полигруппы, полученные из $\mathscr{H}^{*}(X)$ и $\mathscr{H}^{*}(Y)$ сдвигом градуировки на единищу вниз. Тогда $\operatorname{ALT} \mathscr{H}^{* \prime}(X) \leqslant$ $\operatorname{ALT} \mathscr{H}^{*}(X)$ и $\operatorname{ALT} \mathscr{H}^{* \prime}(Y) \leqslant \operatorname{ALT} \mathscr{H}^{*}(Y)$. Множество $A \subset X \times Y$ назовем доминированным, если полигруппа $H^{*}(X \times Y, A)$ почленно факторно доминируется суммой полигрупп $\mathscr{H}^{*}(X) \oplus \mathscr{H}^{*}(Y) \oplus \mathscr{H}^{* \prime}(X) \oplus \mathscr{H}^{* \prime}(Y)$.

И мы будем доказьвать, что вся полигруппа $\mathscr{H}^{*}(X \times Y)$ почленно факторно доминируется этой же суммой полигрупп. Мы докажем для этого, что все подмножества произведения доминированы. Из этого будет вытекать неравенство $\operatorname{ALT} \mathscr{H}^{*}(X \times Y) \leqslant \operatorname{ALT}\left(\mathscr{H}^{*}(X)[\times] \mathscr{H}^{*}(Y)\right)$. Противоположное неравенство вытекает из формулы Кюннета, так как при любом $A \subset X$ и $B \subset Y$ в силу формулы 
Кюннета $H^{*}(X, A) H^{*}(Y, B)=H^{*}(X \times Y, X \times B \cup A \times Y)$. То есть все слагаемые у $\mathscr{H}^{*}(X)[\times] \mathscr{H}^{*}(Y)$ являются также слагаемыми у $\mathscr{H}^{*}(X \times Y)$.

Основной шаг заключается в доказательстве следующей леммы.

Лемма. Пусть даны два замкнутых подмножсества $A, B$ компакта $X$. Тогда $H^{n}(X, A \cap B)$ факторно доминируется суммой пяти групп $H^{n}(X, A) \oplus H^{n}(X, B) \oplus$ $H^{n}(X, A \cup B) \oplus H^{n+1}(X, A) \oplus H^{n+1}(X, B)$.

Доказательство получается рассмотрением отрезка точной последовательности Майера-Виеториса в силу факторного неравенства среднего члена

$$
\begin{aligned}
H^{n}(X, A & \cup B) \rightarrow H^{n}(X, B) \oplus H^{n}(X, A) \\
& \rightarrow H^{n}(X, A \cap B) \rightarrow H^{n+1}(X, A \cup B) \rightarrow H^{n+1}(X, A) \oplus H^{n+1}(X, B) .
\end{aligned}
$$

Назовем $n$-әлементарнылм подмножество произведения $X \times Y$, если оно представляется пересечением $n$ множеств вида $\left(X \times B_{i}\right) \cup\left(A_{i} \times Y\right)$. Индукцией по "индексу элементарности", основываясь на доказанной лемме, получаем, что всякое элементарное множество доминируемо. Далее, произвольное множество является пересечением последовательности элементарных, и его когомологии, являясь прямым пределом доминируемых когомологий, сами доминируемы в силу неравенства $\bigoplus_{i \in I} G_{i} \gg \lim G_{i}$, вытекающего из перестановочности тензорного и периодического произведений с прямым пределом. Итак, мультипликативность тотальных когомологий доказана.

ТеОРема. Отображсние, ставящее в соответствие произвольному конечномерному компакту полигруппу его тотальньх когомологий, индуцирует изоморфизм между размерностной алгеброй и верхней алгеброй Кюннета.

ДокаЗАтельство. Пусть $\operatorname{ALT}_{\mathscr{H}^{*}}(X) \neq \operatorname{ALT}_{\mathscr{H}^{*}}(Y) . \quad$ Tогда Alt $\mathscr{H}^{*}(X) \neq$ Alt $\mathscr{H}^{*}(Y)$ и по теореме о тестировании найдется такая кодировка $C$, что $\| C[+]$ Alt $\mathscr{H}^{*}(X)\|\neq\| C[+]$ Alt $\mathscr{H}^{*}(Y) \|$. В силу теоремы реализации найдется компакт $Z$, для которого $\operatorname{Dim} Z=C$. Тогда в силу мультипликативности тотальных когомологий имеем $\operatorname{dim}(X \times Z)=\operatorname{alt} \mathscr{H}^{*}(X \times Z) \neq \operatorname{alt} \mathscr{H}^{*}(Y \times Z)=\operatorname{dim}(Y \times Z)$, и потому DIM $X \neq \operatorname{DIM} Y$.

Обратно, если $\mathrm{DIM} X \neq \mathrm{DIM} Y$, то для некоторого компакта $Z$ будет $\operatorname{dim}(X \times Z)$ $\neq \operatorname{dim}(Y \times Z)$, откуда alt $\mathscr{H}^{*}(X \times Z) \neq \operatorname{alt} \mathscr{H}^{*}(Y \times Z)$, а значит, и $\operatorname{ALT} \mathscr{H}^{*}(X) \neq$ $\operatorname{ALT} \mathscr{H}^{*}(Y)$. Изотонность отображения $\mathscr{H}^{*}$ вытекает из аддитивности тотальных когомологий, т.е. равенства $\mathscr{H}^{*}(X \cup Y)=\mathscr{H}^{*}(X) \oplus \mathscr{H}^{*}(Y)$, справедливого, если $X \cap Y=\varnothing$. Это завершает доказательство теоремы.

Итог нашим рассмотрениям подводит следуюшая теорема.

ТЕОРЕМА О КОДИРОВАНИИ РАЗМЕРНОСТИ-СВЯЗНОСТИ.

(1) Dim: $\mathfrak{D M} \rightarrow \mathfrak{C D}$ изотонно отображает $\mathfrak{D M}$ на множество кодировок конечной размерности.

(2) Cin: $\mathfrak{C N} \rightarrow \mathfrak{C D}$ изотонно отображает $\mathfrak{C N}$ на множество ненулевых кодировок.

(3) $\operatorname{Dim}(X \times Y)=\operatorname{Dim} X[+] \operatorname{Dim} Y$ для любых компактов.

(4) $\operatorname{Cin}(K \wedge L)=\operatorname{Cin} K\langle+\rangle \operatorname{Dep} L$ для любых комплексов.

(5) $\operatorname{dim} X=\|\operatorname{Dim} X\|$ для любого компакта $X$. 
(6) $\operatorname{cin} K=\langle\langle\operatorname{Cin} K\rangle \partial$ дя любого комплекса $K$.

(7) $X \tau K \Longleftrightarrow \operatorname{Dim} X \leqslant \operatorname{Cin} K$ для любых компакта $X$ и комплекса $K$.

\section{Глава 3. Геометрия}

\section{$\S$ 7. Теоремы об устойчивых пересечениях}

\section{Конечнократные отображения}

Классом компактов назьвается множество компактных подмножеств некоторого пространства. В качестве объемлющего пространства, как правило, выступает гильбертов куб.

Класс компактов назьвается монотоннылм, если всякое замкнутое подмножество компакта из класса принадлежит классу. Класс назьвается аддитивнылм, если он монотонен и содержит конечные объединения своих элементов.

Пусть $f: P \rightarrow \mathbb{R}^{n}$ - отображение полиэдра $P$ с фиксированной на нем триангулящией. Линеаризаиией отображения $f$ назьваем отображение, совпадающее с $f$ на нульмерном остове и линейное на каждом симплексе.

Мы будем говорить, что два пространства $X, Y$ имеют одинаковую классность по отношению к классу пространств $\mathfrak{X}$, если они либо оба принадлежат, либо оба не принадлежат этому классу. Класс компактов в $\mathbb{R}^{n}$ назьвается линейно инвариантнылм, если для любого элемента класса его образ при линейном гомеоморфизме пространства также принадлежит этому классу.

Через $\mathfrak{F}(X, Y)$ обозначается пространство непрерьвных отображений компакта $X$ в пространство $Y$ с компатно-открытой топологией.

АППРОКСИМАЦИОННАЯ ЛЕММА [7]. Для любого компакта $X \subset \mathbb{R}^{n}$ и любого аддитивного, линейно инвариантного класса $\mathfrak{X}$ в пространстве $\mathfrak{F}\left(X, \mathbb{R}^{n}\right)$ всюду плотно множсество отображсений, образы которых имеют с $X$ одинаковую $\mathfrak{X - к л а с с н о с т ь . ~}$

ДокАЗАтельство. Пусть $f: X \rightarrow \mathbb{R}^{n}$ - какое-то непрерьвное отображение и дано $\varepsilon>0$. Не нарушая общности, мы можем предполагать, что $f$ определено на некоторой компактной полиэдральной окрестности $O X$ компакта $X$ в $\mathbb{R}^{n}$. Причем $O X$ является замьканием своей внутренности, т.е. размерно однородным полиэдром. Берем столь мелкую триангулящию окрестности, чтобы линеаризация $f^{\prime}$ отображения $f$ по отношению к этой триангулящии была $\varepsilon / 2$-близка к $f$. Пошевелив отображение $f$, можно получить $\varepsilon / 2$-близкое отображение $f_{1}$, ограничение которого на нульмерном остове в образе дает множество общего положения. Тогда линеаризация отображения $f_{1}$, обозначаемая $f_{1}^{\prime}$, будет $\varepsilon$-близка к $f$, и ограничение ее на любой $n$-мерньй симплекс триангулящии будет гомеоморфизмом. Пусть $T_{1}, \ldots, T_{m}$ - все $n$-мерные симплексы нашей триангулящии. Тогда их объединение дает $O X$. Обозначим через $X_{k}$ пересечение $X \cap T_{k}$. Тогда если $X \in \mathfrak{X}$, то в силу монотонности $X_{k} \in \mathfrak{X}$ при любом $k$. Но $Y_{k}=f_{1}^{\prime}\left(X_{k}\right)$ гомеоморфно $X_{k}$, поскольку $f_{1}^{\prime}$ не вырождено на $n$-мерных симплексах. Поэтому из линейной инвариантности $\mathfrak{X}$ заключаем: $Y_{k} \in \mathfrak{X}$ при любом $k$, и теперь на основании аддитивности $\mathfrak{X}$ заключаем: $f_{1}^{\prime}(X) \in \mathfrak{X}$. Аналогично доказывается, что если $f_{1}^{\prime}(X) \in \mathfrak{X}$, то и $X \in \mathfrak{X}$. 
Класс компактов назьвается топологически инвариантныл, если со всяким компактом содержит и гомеоморфньй ему. Все рассматриваемые в дальнейшем классы компактов по умолчанию предполагаются топологически инвариантными.

Класс компактов назьвается замкнутым, если содержит обратные пределы последовательностей, все члены которых ему принадлежат. Класс компактов назьвается полнылм, если он имеет тип $G_{\delta}$ в пространстве $\exp Q$ всех компактных подмножеств гильбертова куба, рассматриваемого с топологией, порожденной метрикой Хаусдорфа (топологией Виеториса).

Так, класс компактов, экстенсивных относительно данного комплекса $K$, является аддитивньм, замкнутым и полным. Все эти свойства, за исключением полноты, были доказаны в $\S 4$. Докажем полноту. Для комплекса $L$ и пространства $S$ обозначим через $\exp _{L} S$ подпространство $\exp S$ - пространства компактных подмножеств $S$, состояшее из $L$-экстенсивных компактов.

ПоЛНОТА ЭКСПОНЕНТЫ. Для полного метрического сепарабельного $S$ и счетного комплекса L пространство $\exp _{L} S$ полно.

ДокАЗАТЕльство. В силу теоремы Урысона можем считать $S$ подмножеством гильбертова куба $Q$. Поскольку $\exp _{L} S=\exp S \cap \exp _{L} Q$, достаточно доказать полноту $\exp _{L} Q$. Для этого рассмотрим представительную последовательность $\left\{f_{i}\right\}$ частичных отображений из $Q$ в $L$. Для каждого $i$ множество тех компактов в $Q$, на которые продолжается $f_{i}$, открыто. А пересечение последовательности таких множеств как раз и дает $\exp _{L} Q$. Что и требовалось доказать.

ЛЕМма О ПОЛнотЕ. Пусть $\mathfrak{X}$ - некоторый полный класс. Тогда для любого компакта $X$ и любого замкнутого подмножества гильбертова пространства $S$ в пространстве $\mathfrak{F}(X, S)$ множество отображсений, образы которых принадлежсат $\mathfrak{X}$, имеет muп $G_{\delta}$.

ДокаЗАТЕльство. Действительно, отображение $\operatorname{Im}$ из $\mathfrak{F}(X, S)$ ставяшее в соответствие отображению его образ, очевидно, непрерьвно. Множество $\mathfrak{X} \cap \exp S$ имеет тип $G_{\delta}$ как пересечение замкнутого и $G_{\delta}$-множеств, и $\mathfrak{F}(X, S)=\operatorname{Im}^{-1} \mathfrak{X} \cap \exp S$.

ЛЕММА О СОХРАНЕНИИ КЛАССНОСТИ. Пусть $X$ является компактным подмножсеством евклидова пространства $\mathbb{R}^{n}$, принадлежсаци аддитивному полному классу $\mathfrak{X}$. Тогда в $\mathfrak{F}\left(X, \mathbb{R}^{n}\right)$ полно и всюду плотно множсество с образами из $\mathfrak{X}$.

ДокАЗАТЕльство. В силу аппроксимационной леммы в пространстве отображений всюду плотно множество отображений, образы которых принадлежат классу $\mathfrak{X}$. А в силу леммы о полноте это множество имеет тип $G_{\delta}$. Что и требовалось доказать.

Приведенная ниже лемма является небольшой вариацией соответствующего рассуждения из статьи [41].

ЛЕММа О КОНЕЧНОКРАТНОМ ОТОБРАЖЕНИИ. Если образ компакта $X$ при конечнократном отображении принадлежит замкнутому аддитивному классу $\mathfrak{X}$, то и сам компакт принадлежстт этом классу. 
ДокАЗАТЕльство. Пусть компакт $X$ лежит в $n$-мерном кубе $I^{n}$. Обозначим через $\pi_{k}: I^{n} \rightarrow I^{k}$ проекщию куба на его грань, переводящую точку с координатами $\left(x_{1}, \ldots, x_{k}, \ldots, x_{n}\right)$ в точку $\left(x_{1}, \ldots, x_{k}, 0, \ldots, 0\right)$. Для рассматриваемого конечнократного отображения $f: X \rightarrow Y$ обозначим $X_{k}=\left\{\left(\pi_{k} x, y\right) \mid x \in X, y \in Y, f(x)=y\right\}$. Тогда $f$ разлагается в композиции отображений из $X_{k+1}$ в $X_{k}$, если считать, что $X_{0}=Y$. Все отображения этой композиции также конечнократны и, кроме того, параллельны отрезку. Поэтому в конечномерном случае задача сведена к отображениям, параллельным отрезку.

В бесконечномерном случае она также сводится к таким отображениям, только к их бесконечной композиции (обратной последовательности). Этот случай мы оставляем читателю.

Итак, считаем $X \subset Y \times I$ и $f$ - ограничением $Y$-проекции произведения. Прямоугольником будем назьвать подмножество произведения вида $A \times J$, где $J$ - некоторьй связньй промежуток. Множество, являюшееся объединением конечного числа прямоугольников, назьваем мультипрямоугольником. Выберем последовательность замкнутых мультипрямоугольных окрестностей $\left\{O_{n}\right\}$ у компакта $X$ так, чтобы $X=\bigcap_{n=1}^{\infty} O_{n}$. Для каждой такой окрестности определим пространство $Y_{n}$ как факторпространство от $O_{n}$ по разбиению, элементами которого служат компоненты связности пересечений $O_{n} \cap(y \times I), y \in Y$. Тогда имеются естественные отображения из $Y_{n}$ в $Y_{m}$ при $n>m$, и само $Y$ естественно гомеоморфно обратному пределу из $Y_{n}$. Поэтому достаточно доказать, что любое $Y_{n}$ принадлежит $\mathfrak{X}$.

Для каждого прямоугольника $Y^{\prime} \times J$ из $O_{n}$ имеется естественное отображение из его основания $Y^{\prime}$ в $Y_{n}$. А именно, точке $y$ ставится в соответствие та компонента, которая содержит $y \times J$. Образ этого отображения гомеоморфен основанию. Таким образом, все $Y_{n}$ оказывается покрытым конечным числом образов оснований, которые принадлежат классу $\mathfrak{X}$, а значит, и само $Y_{n}$ ему принадлежит.

Следуюшая теорема является вариацией теоремы Дранишникова-Успенского

ТЕОРЕМА О НУЛЬМЕРНОМ ОТОБРАЖЕНИИ [41]. Ecли образ компакта при нульмерном отображсении принадлежсит замкнутому аддитивному классу $\mathfrak{X}$, то и сам компакт принадлежит этому классу.

ДокаЗАТЕльство. Пусть $f: X \rightarrow Y$ - нульмерное отображение компактов. Тогда, как известно, $f$ можно представить в виде обратного предела конечнократных отображений, поэтому теорема вытекает из леммы о конечнократном отображении.

ЛЕММА О КОНЕЧНОКРАТНЫХ ОТОБРАЖЕНИЯХ. В пространстве отображений из компакта $X$ размерности $<n$ в евклидово пространство $\mathbb{R}^{n}$ множсество отображсний кратности $<n$ является всюду плотным типа $G_{\delta}$.

ДокАЗАТЕльство. Скажем, что отображение $f$ является $(n, \varepsilon)$-отображением, если прообраз каждой точки при нем является объединением $n$ дизъюнктных множеств диаметра $<\varepsilon$. Как легко проверить, множество $(n, \varepsilon)$-отображений открыто в пространстве отображений $C\left(X, \mathbb{R}^{n}\right)$. Оно также всюду плотно. Действительно, всякое отображение компакта в $\mathbb{R}^{n}$ апроксимируется композицией $\varepsilon$-отображения из $X$ в полиэдр той же размерности (теорема П. С. Александрова) и кусочно-линейного отображения этого полиэдра в $\mathbb{R}^{n}$. Но всякое общего положения кусочно-линейное отображение полиэдра размерности $<n$ в $\mathbb{R}^{n}$ имеет кратность $<n$. А композиция 
отображения кратности $n$ и $\varepsilon$-отображения является $(n, \varepsilon)$-отображением. Пересечение множеств $(n, \varepsilon)$-отображений, когда $\varepsilon$ пробегает любую стремяшуюся к нулю последовательность, совпадает с множеством отображений кратности $\leqslant n$, что и требовалось доказать.

Эта лемма вместе с теоремой о нульмерных отображениях и леммой о сохранении классности приводит к такому результату.

АППРОКСИМАЦИОННАЯ ТЕОРЕМА. Пусть $\mathfrak{X}-$ аддитивныц̆, полньй, замкнутый класс компактов. Тогда для любого компакта $X \subset \mathbb{R}^{n}$ этого класса в пространстве $\mathfrak{F}\left(X, \mathbb{R}^{n}\right)$ отображсения с образами из $\mathfrak{X}$ составляют полное всюду плотное множество.

\section{Теорема об устойчивых пересечениях}

В этом разделе через $\mathfrak{F}$ мы обозначаем некоторое множество в пространстве отображений $\mathfrak{F}\left(X, \mathbb{R}^{n}\right)$ и называем его классом отображений. Класс $\mathfrak{F}$ называем полнылм, если он имеет тип $G_{\delta}$ и, согласно теореме Александрова, допускает полную метрику.

Если в классе $\mathfrak{F}$ всюду плотно множество тех отображений, образы которых не пересекают некоторого подмножества $Y \subset \mathbb{R}^{n}$, тогда мы будем говорить, что класс $\mathfrak{F}$ устраним с множества $Y$. А множество $Y$ мы будем назьвать в этой ситуации $\mathfrak{F}$-пренебрежимым.

СЧЕТНАЯ АДДИТИВНОСТЬ ПРЕНЕБРЕЖИМОСТИ. Облединение последовательности замкнутых множеств, пренебрежимых некоторым полным классом отображсений $\mathfrak{F}$, является $\mathfrak{F}$-пренебрежсимым.

ДокАЗАТЕльство. Поскольку всякое замкнутое множество в $\mathbb{R}^{n}$ является объединением последовательности компактных, которые также будут $\mathfrak{F}$-пренебрежимьми как подмножества $\mathfrak{F}$-пренебрежимых, постольку утверждение достаточно доказать для компактов. Но множество отображений компакта, образы которых пересекают некоторое компактное множество, замкнуто в пространстве отображений. Поэтому для любого $\mathfrak{F}$-пренебрежимого компакта в классе $\mathfrak{F}$ множество отображений, не пересекающих его, является открытьм и всюду плотным. Теперь ссылка на теорему Бэра завершает доказательство.

Для числа $x$ через $[x]$ обозначим ближайшее к нему целое число. Эта функция однозначно определена для всех чисел, за исключением полущель $x$, каковыми мы называем половины нечетных целых чисел. И положим $\{x\}=|x-[x]|$ для неполуцелых $x$ и $\{x\}=1 / 2$ для полуцелых $x$. Равенство $\{x\}=1 / 2$ характеризует полуцелые числа.

Для набора чисел $x=\left(x_{1}, \ldots, x_{n}\right)$ через $\min _{k} x$ обозначим абсолютную величину $k$-го по абсолютной величине члена этого набора. Так что $\min _{1} x=\min \left\{\left|x_{i}\right|\right\}$ и $\min _{n} x=\max \left\{\left|x_{i}\right|\right\}$.

Точку $x \in \mathbb{R}^{n}$ назовем $k$-(полy) иелой, если она имеет не менее $k$ (полу)целых координат. Ясно, что топологическая размерность множества $k$-цельх точек равна $n-k$. Через $\operatorname{dist}_{0}$ обозначаем метрику на $\mathbb{R}^{n}$, порожденную $\operatorname{sup-нормой~(т.е.~} \operatorname{dist}_{0}(x, y)=$ $\left.\max \left|x_{i}-y_{i}\right|\right)$. 
ЛЕМма ОБ ОКРУГЛЕНИИ. Если компакт $X \subset \mathbb{R}^{n}$ не содержит $k$-полуцелых точек, то он допускает непрерывный сдвиг в $(n-k+1)$-иелье точки, смещающий его точки в метрике dist $_{0}$ не далее чем на половину.

ДоКАЗАТЕльство. Для $x \in \mathbb{R}^{n}$ положим $\{x\}=\left(\left\{x_{1}\right\}, \ldots,\left\{x_{n}\right\}\right)$. Для любой точки $x \in X$ имеем $\min _{n-k+1}\{x\}<1 / 2$. Действительно, предположение противного означало бы, что $\left\{x_{i}\right\}=1 / 2$ для $k$ координат, что означало бы $k$-полуцелость $x$. Определим теперь “округляющее” отображение $s: X \rightarrow \mathbb{R}^{n}$ следующим образом. Если $x_{i} \leqslant \min _{k}\{x\}$, то $s(x)_{i}=\left[x_{i}\right]$ (определение корректно в силу только что доказанного $)$, иначе $s(x)_{i}=\left(2 \min _{k}\{x\}\right) x_{i}+\left(1-2 \min _{k}\{x\}\right)\left[x_{i}\right]$. Последнее определение корректно и для полуцелых $x_{i}$, потому что в этом случае обращается в нуль коэффищиент при $\left[x_{i}\right]$. Тогда покоординатное различие между $s(x)$ и $x$ не превосходит половины, а образ $s(X)$ состоит из $(n-k+1)$-целых точек.

Доказательство следующего утверждения, по существу, принадлежит Г. С. Чогошвили $[42] .^{5}$

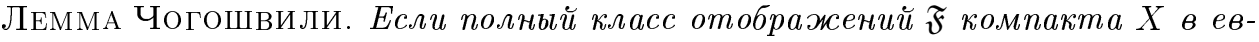
клидово пространство $\mathbb{R}^{n}$ устраним со всякой плоскости размерности $k$, то почти все отображсения этого класса имеют образ размерности меньше чем $n-k$.

ДокАЗАТЕльство. Обозначим через $R(n, k)$ множество $k$-полуцелых точек пространства $\mathbb{R}^{n}$. Для положительного $c$ положим $c R(n, k)=\{c x \mid x \in R(n, k)\}$. Тогда объединение множеств $\bigcup_{m \in \mathbb{N}} \frac{1}{m} R(n, k)$ является $\mathfrak{F}$-пренебрежимым как объединение счетного числа $k$-мерных плоскостей.

Таким образом, для доказательства леммы достаточно убедиться, что всякий компакт, лежащий в дополнении к этому объединению, имеет размерность $<n-k$. А для этого достаточно убедиться в том, что он допускает сколь угодно малые сдвиги в компакты размерности $\leqslant n-k-1$. Но всякий компакт, лежаший в дополнении к $c R(n, k)$, допускает $c$-сдвиг (в метрике dist $\left._{0}\right)$ в $(n-k-1)$-мерньй полиэдр, как это следует из леммы об округлении.

Гиперплоскость в $\mathbb{R}^{n}$, задаваемая уравнением $x_{i}=0$, называется координатной. И координатной плоскостью назьвается пересечение координатных гиперплоскостей. Как заметил Понтрягин, при доказательстве леммы Чогошвили используется лишь устранимость с плоскостей, параллельных координатным.

Поэтому если некоторое семейство $k$-мерных плоскостей $\mathfrak{L}$ параллельно инвариантно, т.е. со всякой плоскостью содержит все ей параллельные и содержит все координатные плоскости, то в силу замечания Понтрягина $\mathfrak{L}$-устранимости достаточно для справедливости заключения леммы Чогошвили.

А так как набор плоскостей, достаточно близкий к координатному, сам является координатным в некоторой системе координат, то замечание Понтрягина позволяет доказать следуюшее уточнение леммы Чогошвили.

\footnotetext{
${ }^{5}$ Сам Чогошвили в цитированной работе формулировал и пытался доказать неверное утверждение, что компакт, устранимый с любой плоскости размерности $k$ в $\mathbb{R}^{n}$, имеет размерность $<(n-k)$. Контрпример к этому утверждению построен А. Н. Дранишниковым [43].
} 
УТОЧНЕННАЯ ЛЕММА ЧОГОШВИЛИ. Если полный класс отображсений $\mathfrak{F}$ некоторого компакта в $\mathbb{R}^{n}$ устраним со всякой плоскости, принадлежащей некоторому параллельно инвариантному и плотному множеству $k$-мерных плоскостей, то почти все отображения класса имеют размерность образа меньше чем $n-k$.

Устойчивые пересечения. Пусть даны классы отображений $\mathfrak{F}_{i}: X_{i} \rightarrow S$. Набор отображений $\left\{f_{i}: X_{i} \rightarrow S\right\}$ называется устойчиво пересекающимся относительно классов $\mathfrak{F}_{i}$, если $f_{i} \in \mathfrak{F}_{i}$ и для всяких достаточно близких к ним отображений $f_{i}^{\prime} \in \mathfrak{F}_{i}$ непусто пересечение $\bigcap_{i} f_{i}^{\prime}\left(X_{i}\right)$.

И мы говорим, что отображение $f \in \mathfrak{F}$ устойчиво по отношению к $\mathfrak{F}$ пересекает некоторое множество, если всякое достаточно близкое к $f$ отображение этого класса также пересекает это множество.

Пусть дан набор классов $\left\{\mathfrak{F}_{i}\right\}_{i \leqslant k}$ отображений компактов $X_{1}, X_{2}, \ldots, X_{k}$ в $\mathbb{R}^{n}$. Их произведением $\prod \mathfrak{F}_{i}$ назьвается класс непрерьвных отображений из произведения $X_{1} \times X_{2} \times \cdots \times X_{k}$ в пространство $\mathbb{R}^{n k}$ с фиксированной на нем структурой произведения $\mathbb{R}^{n k}=\mathbb{R}^{n} \times \mathbb{R}^{n} \times \cdots \times \mathbb{R}^{n}$, имеющих вид прямых произведений $f_{1} \times f_{2} \times \cdots \times f_{k}$. Очевидно, что произведение полных классов полно.

Плоскость $L$ размерности $n$ в произведении $\mathbb{R}^{n k}$ будем назьвать косой, если ее проекция накрьвает любой из множителей этого произведения. Всякая косая плоскость при любой паре $i, j \leqslant k$ определяет линейньй гомеоморфизм $L_{i j}: \mathbb{R}^{n} \rightarrow \mathbb{R}^{n}$, определяемьй следующим способом: для $x \in \mathbb{R}^{n}$ выбираем точку $y \in L$, проекция которой на $i$-ю координату равна $x$, и в качестве $L_{i j}(x)$ берем проекцию $y$ на $j$-ю координату. Набор классов отображений называют устойчиво пересекающимся, если из каждого класса можно выбрать по представителю и получить устойчиво пересекающийся по отношению к этим классам набор отображений. И мы говорим, что класс отображений устойчиво пересекает множество, если хотя бы один представитель этого класса устойчиво пересекает это множество.

ЛЕММА О КОСОЙ ПЛОСКОСТИ. Продуктивный класс $\mathfrak{F}=\mathfrak{F}_{1} \times \mathfrak{F}_{2} \times \cdots \times \mathfrak{F}_{n}$ устойчиво пересекает некоторую косую плоскость $L$ в том и только том случае, когда устойчиво пересекаются классь отображений $L_{i 1} \circ \mathfrak{F}_{i}$.

ДокАЗАТЕЛЬство. Пересечение образа отображения $f_{1} \times f_{2} \times \cdots \times f_{k}$ с $L$, как нетрудно понять, проектируется на первый множитель как раз на пересечение образов композиций $L_{i 1} f_{i}$. Поэтому эти множества пусты или непусты одновременно. Откуда и следует нужное нам утверждение.

ТЕОРЕМА ОБ УСТОЙЧИВОМ ПЕРЕСЕЧЕНИИ. Пусть дан набор компактов $X_{1}, \ldots, X_{k}$ размерностей $<n$, размерность произведения которьх $\operatorname{dim} \prod X_{i} \geqslant$ $n k-n$, тогда найдутся такие отображсения $f_{i}: X_{i} \rightarrow \mathbb{R}^{n}$, которые в совокупности устойчиво пересекаются.

ДокАЗАТЕЛЬСТво. Обозначим через $\mathfrak{F}_{i}: X_{i} \rightarrow \mathbb{R}^{n}$ полньй класс непрерывных отображений из $X_{i}$ в $\mathbb{R}^{n}$, имеюших кратность $<n$ (см. лемму о конечнократных отображениях). Продуктивный класс $\prod \mathfrak{F}_{i}$ отображений в $\mathbb{R}^{n k}$ обозначим $\mathfrak{F}$. Так как 
размерность образа при нульмерных отображениях не понижается (теорема о нульмерных отображениях), то образы всех отображений класса $\mathfrak{F}$ имеют размерность $\geqslant n k-n$. Поскольку множество косых $n$-мерных плоскостей в $\mathbb{R}^{n k}$ параллельно инвариантно и всюду плотно, постольку уточненная лемма Чогошвили позволяет заключить, что найдется косая плоскость размерности $n$, которую класс $\mathfrak{F}$ устойчиво пересекает. Это значит, что найдутся линейные гомеоморфизмы $L_{1}, \ldots, L_{n}$, для которых устойчиво относительно $\mathfrak{F}_{i}$ пересекаются отображения $L_{i} f_{i}$ (лемма о косой плоскости). Но почти все непрерывные отображения из $X_{i}$ в $\mathbb{R}^{n}$ принадлежат $\mathfrak{F}_{i}$ (лемма о конечнократных отображениях), поэтому устойчивость относительно $\mathfrak{F}_{i}$ влечет устойчивость относительно класса всех непрерьвных отображений. Что и требовалось доказать.

\section{Теорема о непренебрежимости}

Поликомпакты. Конечньй упорядоченньй набор компактов $\mathscr{X}=\left\{X_{1}, \ldots, X_{n}\right\}$ будем называть поликомпактом. При этом компакт $X_{i}$ назьваем $i$-й компонентой поликомпакта $\mathscr{X}$. Поликомпакты обозначаем рукописными буквами $\mathscr{X}, \mathscr{Y}$, а их компоненты соответствуюшими печатными $X_{i}, Y_{j}$. Через $\prod \mathscr{X}$ обозначаем произведение $X_{1} \times X_{2} \times \cdots \times X_{n}$ компонент поликомпакта.

Число компактов, составляюших данньй поликомпакт, назьваем длиной поликомпакта. Размерностью поликомпакта $\mathscr{X}=\left\{X_{i}\right\}_{1 \leqslant i \leqslant m}$ назьвается максимум $\operatorname{dim} X_{i}$; обозначение: $\operatorname{dim} \mathscr{X}$.

Для двух поликомпактов $\mathscr{X}$ и $\mathscr{Y}$ через $\mathscr{X}, \mathscr{Y}$ обозначаем составной поликом$n a \kappa m$, полученный объединением поликомпактов $\mathscr{X}$ и $\mathscr{Y}$, порядок на котором задается так, что любая $\mathscr{X}$-компонента предшествует любой $\mathscr{Y}$-компоненте, порядок $\mathscr{X}$-компонент такой же, как в $\mathscr{X}$, а $\mathscr{Y}$-компонент - такой же, как в $\mathscr{Y}$.

И будем назьвать отображением $F: \mathscr{X} \rightarrow S$ поликомпакта $\mathscr{X}=\left\{X_{1}, \ldots, X_{m}\right\}$ в пространство $S$ совокупность отображений $F=\left\{f_{i}: X_{i} \rightarrow S\right\}$.

Пространство отображений поликомпакта $\mathscr{X}=\left\{X_{1}, \ldots, X_{m}\right\}$ естественно отождествляется с произведением $\prod_{i=1}^{m} \mathfrak{F}\left(X_{i}, S\right)$ и рассматривается с топологией произведения. А расстояние между отображениями определяется как максимум из расстояний между их соответствуюшими компонентами: $\operatorname{dist}(F, G)=\max \operatorname{dist}\left(f_{i}, g_{i}\right)$.

Для отображения $F=\left\{f_{i}: X_{i} \rightarrow S\right\}_{1 \leqslant i \leqslant m}$ поликомпакта $\mathscr{X}$ определим его пересеченный образ $F^{\cap}(\mathscr{X})$ как пересечение $F^{\cap}(\mathscr{X})=\bigcap_{1 \leqslant i \leqslant m} f_{i}\left(X_{i}\right)$.

Будем говорить, что поликомпакт $\mathscr{X}$ не имеет устойчивых пересечений в пространстве $S$, если всякое отображение из $\mathscr{X}$ в $S$ аппроксимируется отображением с пустьм пересеченным образом. И будем говорить, что поликомпакты $\mathscr{X}=\left\{X_{1}, \ldots, X_{n}\right\}$ и $\mathscr{Y}=\left\{Y_{1}, \ldots, Y_{m}\right\}$ не имеют устойчивых пересечений в пространстве $S$, если устойчивых пересечений в $S$ не имеет составной поликомпакт $\mathscr{X}, \mathscr{Y}$. В противном случае мы будем говорить, что поликомпакты $\mathscr{X}$ и $\mathscr{Y ~ и м е ю т ~ у с т о и ̆ ч и в ы е ~ п е р е с е ч е н и я ~ в ~} S$ или что $\mathscr{X}$ и У устойчиво пересекаются в $S$.

И мы назьваем множество $R \subset S$ пренебрежимым относительно поликомпакma $\mathscr{X}$, если всякое отображение $F: \mathscr{X} \rightarrow R$ аппроксимируется отображениями, пересеченный образ которых не пересекает $R$.

Следуюшая лемма для компактов была доказана Торуньчиком [7]. 
ЛЕмма ТорУньчИКА. Если поликомпакты $\mathscr{X}$ и У्У не имеют устойчивых пересечений в полном метрическом пространстве $S$, то пересеченный образ почти всех отображений поликомпакта $\mathscr{X}$ является $\mathscr{Y - п р е н е б р е ж и м ы м . ~}$

ДоКАЗАТЕЛЬСТвО. Пусть $\left\{F_{i}\right\}$ и $\left\{G_{i}\right\}$ - всюду плотные последовательности отображений из $\mathscr{X}$ и $\mathscr{Y}$ в $S$. С помошью бесконечного процесса мы “пошевелим" все эти отображения таким образом, чтобы “пошевеленные" отображения $F_{i}^{\prime}$ и $G_{i}^{\prime}$ обладали следуюшими свойствами:

(1) $\operatorname{dist}\left(F_{i}, F_{i}^{\prime}\right) \leqslant 1 / 2^{i}$ и $\operatorname{dist}\left(G_{i}, G_{i}^{\prime}\right) \leqslant 1 / 2^{i}$ (близость);

(2) $F_{j}^{\prime \cap}(\mathscr{X}) \cap G_{k}^{\prime \cap}(\mathscr{Y})=\varnothing$ при любых $j, k$ (дизъюнктность).

Покажем, как из существования пошевеленной последовательности вытекает утверждение леммы. Прежде всего, заметим, что пошевеленные последовательности ввиду условия близости сохраняют свойства всюду плотности, которыми обладали первоначальные.

Для любого отображения $F_{k}^{\prime}$ в пространстве отображений поликомпакта $\mathscr{У ~ о б о - ~}$ значим через $C_{k}$ множество тех из них, для которых пересеченный образ не пересекается с $F_{k}^{\prime \cap} \mathscr{X}$. Множество $C_{k}$ открыто и всюду плотно, потому что содержит последовательность $G^{\prime}{ }_{i}$. Таким образом, пересечение всех $C_{k}$ будет всюду плотным $G_{\delta}$-множеством. Но любой элемент этого пересечения имеет пересеченньй образ, дизъюнктный с пересеченными образами всех $F_{k}^{\prime \cap}$. А всякое множество, лежашее в дополнении ко всем пересеченным образам последовательности $G_{k}^{\prime}$, является $\mathscr{Y}$-пренебрежимым, ибо последовательность отображений $G_{k}^{\prime}$ всюду плотна.

Алгоритм шевеления выглядит так. Введем понятие текущего $k$-го отображения составного поликомпакта $\mathscr{X}, \mathscr{Y}$. Первоначально оно совпадает с $F_{k}, G_{k}$. В процессе построения оно меняется. А конечньй результат - отображения $F^{\prime}, G^{\prime}$, будет получаться как предел последовательности соответствующих текуших отображений.

На первом шаге мы заменяем первое текущее отображение на $\varepsilon_{1}$-близкое с пустым пересеченным образом. И запоминаем число $\delta_{1}-$ меру дизғюнктной устойчивости этого отображения, которое обладает тем свойством, что всякое $\delta_{1}$-близкое к нему отображение также имеет пустой пересеченньй образ.

На $k$-м шаге мы заменяем все текушие отображения с первого по $k$-е на $\varepsilon_{k}$-близкие и обладающие тем свойством, что при любых $i, j \leqslant k$ дизъюнктны пересеченные образы $i$-го текущего отображения поликомпакта $\mathscr{X}$ и $k$-го текущего отображения поликомпакта $\mathscr{Y . ~ И ~ з а п о м и н а е м ~ м е р у ~ у с т о и ̆ ч и в о с т и ~} \delta_{k}$ этой ситуации.

Возможность замены любого текущего отображения и даже любой конечной совокупности таких отображений на сколь угодно близкие отображения с дизъюнктными пересеченными образами вытекает из условия неустойчивости пересечений.

Построение описано. Осталось описать, как выбирать $\varepsilon_{k}$, чтобы при предельном переходе сохранить свойства дизъюнктности пересечений, которых мы добились для текуших отображений. Для этого достаточно выбирать $\varepsilon_{k}$ таким образом, чтобы при любом $n$ вьполнялось неравенство $\sum_{i=n+1}^{\infty} \varepsilon_{i} \leqslant \delta_{n} / 2$. Чтобы удовлетворить этому условию, достаточно выбирать $\varepsilon_{k}$ не превосходяшим $\delta_{k-i} / 3^{i}$. Лемма Торуньчика доказана.

Условие отсутствия устойчивых пересечений для отображений поликомпактов $\mathscr{X}$ 
и $\mathscr{Y ~ р а в н о с и л ь н о ~ п р е н е б р е ж и м о с т и ~ п р о с т р а н с т в а ~} S$ составным поликомпактом $\mathscr{X}, \mathscr{Y}$. Такая переформулировка позволяет обобшить лемму Торуньчика следующим образом.

ОТНОСИТЕЛЬНАЯ ЛЕМмА ТОРУНЬЧИКА. Пусть $\mathscr{X}, \mathscr{Y}$ - два поликомпакта, $S$ - полное метрическое пространство и $A$ - замкнутое подпространство $S$. Тогда если $A$ является пренебрежимым для составного поликомпакта $\mathscr{X}, \mathscr{Y}$, то для почти всех отображсений поликомпакта $\mathscr{X}$ пересечение их пересеченных образов с А является У-пренебрежимым.

Доказательство относительной версии леммы Торуньчика, по сушеству, не отличается от приведенного вьше доказательства абсолютной и предоставляется читателю. Из леммы Торуньчика и апшроксимационной леммы получаем такое следствие.

ТЕОРЕМА О НЕПРЕНЕБРЕЖИМОСТИ. Если поликомпакт $\left\{X_{0}, X_{1}, \ldots, X_{m}\right\}$ uмeет в $\mathbb{R}^{n}$ устойчивые пересечения $и X_{0} \subset \mathbb{R}^{n}$, то $X_{0}$ не пренебрежсим поликомпактом $X_{1}, \ldots, X_{m}$.

ДокАЗАТЕльство. Рассмотрим класс компактов, являюшихся пренебрежимыми по отношению к поликомпакту $X_{1}, \ldots, X_{n}$. Он аддитивен и линейно инвариантен. Предполагая $X_{0}$ пренебрежимьм поликомпактом $X_{1}, \ldots, X_{m}$, рассмотрим произвольное отображение $f_{0}, \ldots, f_{m}: X_{0}, \ldots, X_{m} \rightarrow \mathbb{R}^{n}$. Отображение $f_{0}$ имеет апшроксимацию $f_{0}^{\prime}$ с образом, пренебрежимым поликомпактом $X_{1}, \ldots, X_{n}$ в силу апшроксимационной леммы. Тогда, взяв аппроксимацию $f_{1}^{\prime}, \ldots, f_{m}^{\prime}$, пересечение образов которой минует $f_{0}^{\prime}\left(X_{0}\right)$, получим в итоге отображение из $X_{0}, \ldots, X_{m}$ с пустым пересеченньм образом, аппроксимирующее исходное. То есть мы доказали, что устойчивых пересечений у компактов $X_{0}, \ldots, X_{m}$ в $\mathbb{R}^{n}$ нет, вопреки предположению.

\section{$\S$ 8. Верхние оценки размерности пересечения}

\section{Леммы о множествах дикости}

КРИТЕРИЙ $n$-ПРЕНЕБРЕЖИМОСТИ. Для сигма-компактного подмножества $X \subset \mathbb{R}^{n}$ и иелого $k \in[0, n]$ следующие два условия әквивалентны:

(1) $X$ пренебрежимо $k$-шаром;

(2) $X$ пренебрежимо любым компактом размерности $\leqslant k$.

ДокаЗАТЕльство. Пусть выполнено первое условие. Докажем, что тогда $X$ пренебрежимо полиэдром $P$ размерности $\leqslant k$. Действительно, при данных условиях в пространстве отображений $\mathfrak{F}\left(P, \mathbb{R}^{n}\right)$ множество тех, ограничение которых на данньй симплекс не пересекает $X$, всюду плотно (в силу пренебрежимости $X$ шаром размерности $k$ ) и полно (так как $X$ - сигма-компактно). Поэтому и пересечение таких множеств по всем симплексам полиэдра также имеет эти свойства. А всюду плотность этого пересечения как раз и означает $P$-пренебрежимость для $X$.

Если теперь $Y$ - компакт размерности $\leqslant k$, то всякое отображение из $Y$ в $\mathbb{R}^{n}$ приближается, в силу теоремы Александрова, отображением, образом которого служит полиэдр $P$ той же размерности. А последний мальм шевелением снимается с $X$ в силу вьшедоказанного. Что и позволяет доказать $Y$-пренебрежимость множества $X$. 
Множества евклидова пространства, удовлетворяюшие условиям доказанного вьше предложения, называются пренебрежимылми в размерности $n$ или, короче, n-пренебрежимыми.

Следующее утверждение, по существу, принадлежит Франклю [44].

ТЕОРема ФранкЛя. Компакт $X \subset \mathbb{R}^{n}$ имеет размерность $\operatorname{dim} X<n-1$ в том и только том случае, когда является 1-пренебрежимым.

ДоКАЗАТЕЛЬСТво. Если $\operatorname{dim} X \geqslant n-1$, то $\operatorname{dim} X \times I \geqslant n$, и по теореме об устойчивых пересечениях сушествуют устойчивые пересечения $X$ и $I$ в $\mathbb{R}^{n}$, а по теореме о непренебрежимости отсюда следует $I$-непренебрежимость $X$.

Если же компакт $X$ разбивает пространство, то одна из компонент разбиения ограничена. Обозначим ее $C$. Тогда параллельными переносами можно устроить счетное покрытие пространства копиями множества $C$, обозначим это покрытие $\omega$. Тогда сжимаюшие гомототии дадут последовательность $\varepsilon_{i}$-покрытий $\omega_{i}$ для последовательности $\varepsilon_{i} \rightarrow 0$. Тогда объединение границ элементов покрытий будет иметь размерность такую же, как гранища у $C$, т.е. $<n-1$, а дополнение будет нульмерно. И мы получим противоречие с неравенством Урысона.

ЛЕММА О НУЛЬМЕРНОМ ПЕРЕСЕЧЕНИИ С ДИСКОМ. Ecли сигма-компакт $X \subset \mathbb{R}^{n}$ имеет размерность $\operatorname{dim} X<n-1$ и $n>3$, то почти все отображсения двумерного диска $f: D \rightarrow \mathbb{R}^{n}$ имеют нульмерное и 2-пренебрежимме пересечение $f(D) \cap X$.

ДокАЗАТЕльство. Начнем с замечания, что случай сигма-компактного $X$ вытекает из случая компактного $X$. Действительно, пусть $X_{i}$ - последовательность компактов, дающая в объединении $X$. Обозначим через $\mathfrak{F}_{i}$ множество отображений из $D$ в $\mathbb{R}^{n}$, для которых пересечение с $X_{i}$ нульмерно и 2-пренебрежимо. Если мы знаем, что дополнение к $\mathfrak{F}_{i}$ первой категории, то почти все отображения из $\mathfrak{F}\left(D, \mathbb{R}^{n}\right)$ принадлежат пересечению $\mathfrak{F}_{i}$. Но отображения из этого пересечения имеют нульмерное пересечение с $X$, ибо объединение последовательности нульмерных компактов нульмерно, и имеют 2-пренебрежимые пересечения с $X$, ибо объединение последовательности 2-пренебрежимых компактов 2-пренебрежимо. Итак, в дальнейшем считаем $X$ компактом.

В пространстве отображений диска в $\mathbb{R}^{n}$ множество $\mathfrak{F}_{\varepsilon}$ отображений, имеющих компоненту связности у пересечения образа с $X$, диаметра не больше некоторого $\varepsilon$, замкнуто. Докажем нигде не плотность этого множества. Пусть дано $\varepsilon>0$. Зафиксируем столь мелкую триангуляцию на диске, чтобы образы симплексов при $f$ имели диаметр меньше чем $\varepsilon$. Тогда в силу 1 -пренебрежимости $X$ найдется $\varepsilon$-близкое отображение $f^{\prime}$, при котором образ одномерного остова триангуляции не пересекает $X$. Если $n>4$, то дальше мы, просто приводя в общее положение отображение $f^{\prime}$, получаем вложение. Но тогда пересечения образов различных симплексов триангулящии с $X$ дизъюнктны и имеют диаметр не больше $3 \varepsilon$.

Если же $n=4$, то приведенное в общее положение отображение $f^{\prime}$ имеет конечное число двойных точек. Тогда малой изотопией всего пространства мы сдвигаем двойные точки с $X$. В результате получаем, что пересечения образов симплексов у произотопированного отображения с $X$ дизъюнктны. 
В силу теоремы Бэра получаем, что множество $\mathfrak{F}_{0}$, состоящее из отображений, не принадлежащих $\mathfrak{F}_{\varepsilon}$ ни при каком $\varepsilon>0$, является всюду плотным $G_{\delta}$-множеством в пространстве отображений, а его элементами как раз являются отображения с нульмерным пересечением.

Для почти всех пар отображений диска в $\mathbb{R}^{n}$ их пересечение с $X$ пусто. Действительно, поскольку множество пар с пустым пересечением открыто, достаточно доказать его всюду плотность. Для этого можно ограничиться рассмотрением кусочно-линейных отображений. Но кусочно-линейные отображения в общем положении пересекаются по конечному числу точек (если $n=4)$, и эти точки в общем положении не лежат на $X$, ввиду нигде не плотности последнего. Далее применяем уточненную лемму Торуньчика к отображениям набора $D, D$ в $\mathbb{R}^{n}$ относительно $X$ и получаем, что для почти всех отображений из $D$ в $\mathbb{R}^{n}$ пересечение образа с $X$ является 2-пренебрежимым. В таком случае для почти всех отображений мы имеем одновременно 2-пренебрежимость и нульмерность пересечения образа отображения с $X$.

ЛЕММА ОБ ОДНОМЕРНОМ ПЕРЕСЕЧЕНИИ С ДИСКОМ. Ecлu cuгмa-Комnaкm $X \subset \mathbb{R}^{n}$ имеет размерность $\operatorname{dim} X<n \quad u n>3$, то почти все отображения двумерного диска $f: D \rightarrow \mathbb{R}^{n}$ имеют одномерное и 2-пренебрежимое пересечение $f(D) \cap X$.

ДокАЗАтельство. Редукция сигма-компактного $X$ к компактному вьполняется так же, как и в предыдущей лемме. Поэтому считаем $X$ компактом. Выберем в диске $D$ всюду плотную последовательность $\left\{x_{i}\right\}$. Тогда для любого $i$ множество $\mathfrak{F}_{i}$ тех отображений из $D$ в $\mathbb{R}^{n}$, для которых образ $x_{i}$ не принадлежит $X$, является открытым и всюду плотньм в пространстве отображений $\mathfrak{F}\left(D, \mathbb{R}^{n}\right)$. При $f \in \bigcap \mathfrak{F}_{i}$ будет $\operatorname{dim} f^{-1}(X) \leqslant 1$, т.е. это вьполняется для почти всех отображений.

Далее, для почти всех отображений из $\mathfrak{F}\left(D, \mathbb{R}^{n}\right) f^{-1}(X)$ содержится в множестве точек однократности. Действительно, пусть $D_{1}$ и $D_{2}$ - два непересекаюшихся рациональных (т.е. с рациональньми центрами и радиусами) круга в $D$; аналогично отмеченному при доказательстве предыдущей леммы, для почти всех отображений из $D$ в $\mathbb{R}^{n}$ пересечение образов этих дисков с $X$ пусто. Так как рациональных кругов счетное количество, то для почти всех отображений и любых непересекающихся рациональных кругов пересечение их образов с $X$ пусто. Но отображения такого типа не имеют на $X$ точек неоднократности. Поэтому для почти всех отображений из $\mathfrak{F}\left(D, \mathbb{R}^{n}\right)$ будет $\operatorname{dim} f(D) \cap X \leqslant 1$. Наконец то, что для почти всех отображений диска их пересечение с $X$ является 2-пренебрежимьм, доказывается так же, как в лемме о нульмерном пересечении.

Ручные компакты. Компакт $X$ в $\mathbb{R}^{n}$ размерности $\leqslant n-3$ называется ручнылм, если для всякого односвязного открытого множества $U$ односвязна разность $U \backslash X$.

ЛЕММА О 2-ПРЕНЕБРЕЖИМОСТИ. Всякий 2-пренебрежимый компакт яляется ручным.

ДокАЗАТЕльство. Пусть $U$ - односвязное открытое множество. Пусть $f: S^{1} \rightarrow$ $U \backslash X-$ произвольная петля. Пусть $\varepsilon$ столь мало, что всякая $\varepsilon$-близкая к ней петля ей гомотопна. Продолжим $f$ до отображения $F: D \rightarrow U$. Это возможно в силу односвязности $U$. Пошевелим $F$ и получим $\varepsilon$-близкое отображение $F^{\prime}$, для которого 
образ не пересекает $X$, что возможно в силу 2-пренебрежимости последнего. Тогда $F^{\prime}$ осуществляет гомотопию нулю своего ограничения на край, которое по выбору $\varepsilon$ гомотопно $f$. Односвязность $U \backslash X$ доказана.

ЛЕММА О НУЛЬМЕРНОМ МНОЖЕСТВЕ ДИКОСТИ. Для всякого сигма-компакта $X$ размерности $\operatorname{dim} X<n-1$, лежсащего в $\mathbb{R}^{n}, n>3$, найдется такое нульмерное 2-пренебрежсимое $F_{\sigma}$-множество $Z$ (множество дикости), что всякий компакт $C \subset X \backslash Z$ является 2-пренебрежимым.

ДокАЗАТЕльство. Опираясь на лемму о пересечении с диском, в пространстве всех отображений двумерного диска в $\mathbb{R}^{n}$ выбираем всюду плотную последовательность отображений $f_{i}$, образы которых имеют нульмерные и 2-пренебрежимые пересечения с $X$. Пусть $Z$ является объединением пересечений $f_{i}(D) \cap X$. Тогда любой компакт из дополнения $X \backslash Z$ является $D$-пренебрежимым и потому ручным.

ЛЕММА ОБ ОДНОМЕРНОМ МНОЖЕСТВЕ ДИКОСТИ. Для всякого сигма-компакта $X$ размерности $\operatorname{dim} X<n$, лежащего в $\mathbb{R}^{n}, n>3$, найдется такое одномерное 2-пренебрежимое $F_{\sigma}$-множество $Z$ (множество дикости), что всякий компакт $C \subset X \backslash Z$ является 2-пренебрежимым.

ДоКАЗАТЕЛЬСТВО. Эта лемма вьводится из леммы об одномерном пересечении с диском точно так же, как предыдущая из леммы о нульмерном пересечении.

\section{Верхние оценки парных пересечений}

Результаты этого раздела будут усилены в следующем.

ЛЕММА ОБ ЭКСТЕНСИВНОСТИ ДОПОЛНЕНИЯ. Ecли $X-$ компакт в шаре $B^{n}$ размерности $\operatorname{dim} X<n-2$ - имеет в шаре односвязное дополнение и $Y$ является компактом, для которого $\operatorname{dim} X \times Y<n$, то $Y \tau\left(B^{n} \backslash X\right)$.

ДоКАЗАТЕЛЬСТво. Пусть $\mathscr{H}^{*}(X), \mathscr{H}^{*}(Y)$ обозначают тотальные когомологии компактов $X$ и $Y$ соответственно. Тогда

$$
\text { alt } \mathscr{H}^{*}(Y) H^{*}\left(X, \partial B^{n} \cap X\right) \leqslant \operatorname{alt} \mathscr{H}^{*}(X) \mathscr{H}^{*}(Y)=\operatorname{dim} X \times Y<n .
$$

Полигрупша гомологий $H_{*}\left(B^{n} \backslash X\right)$ является $n$-двойственной полигрупе когомологий $H^{*}\left(X, \partial B^{n} \cap X\right)$ в силу двойственности Александера. По критерию двойственности для полигрупш отсюда следует совместимость $\mathscr{H}^{*}(Y) \tau H_{*}\left(B^{n} \backslash X\right)$. Что означает $\operatorname{Dim} Y \leqslant \operatorname{Cin} B^{n} \backslash X$ в силу теоремы о кодировании и влечет $Y \tau B^{n} \backslash X$ в силу критерия экстенсивности Дранишникова.

Следуюший результат Дранишникова играет ключевую роль в дальнейших рассмотрениях.

КРИТЕРИЙ ПРЕНЕБРЕЖИМОСТИ КОМПАКТОМ. Ручной компакт $X$ размерности меньше чем $n-2$, лежсащий в $\mathbb{R}^{n}$, пренебрежим компактом $Y$, если и только если $\operatorname{dim} X \times Y<n$. 
ДоКАЗАТЕльСТво. Если $\operatorname{dim} X \times Y \geqslant n$, то в силу теоремы об устойчивом пересечении компакты $X$ и $Y$ устойчиво пересекаются в $\mathbb{R}^{n}$, а потому $X$ не является $Y$-пренебрежимым в силу теоремы о непренебрежимости. Необходимость условия на размерность произведения тем самым доказана.

Доказательство достаточности ведется индукцией по размерности компакта $X$. Пусть $\operatorname{dim} X=k+1$ и теорема доказана для компактов размерности $k$. Рассмотрим разложение Урысона $X=X_{k} \cup X_{0}$, где $X_{k}$ типа $F_{\sigma}$ и $\operatorname{dim} X_{k}=k, \operatorname{dim} X_{0}=0$. Тогда в силу индукционного предположения и счетной аддитивности пренебрежимости имеем, что для почти всех отображений $f: Y \rightarrow \mathbb{R}^{n}$ пересечение $f(Y) \cap X$ нульмерно. Далее, как мы знаем, почти все отображения из $Y$ в $\mathbb{R}^{n}$ конечнократны. Поэтому нам достаточно показать, что конечнократные отображения, нульмерно пересекающие $X$, аппроксимируются отображениями с пустым пересечением.

Итак, дано конечнократное отображение $f: Y \rightarrow \mathbb{R}^{n}$, для которого $\operatorname{dim} f(Y) \cap$ $X=0$, и дано $\varepsilon>0$. Обозначим через $Y^{\prime}$ прообраз $f^{-1} X$. Тогда $\operatorname{dim} Y^{\prime}=0$ в силу конечнократности $f$ и найдется такое конечное дизъюнктное покрытие для $Y^{\prime}$ замкнутыми подмножествами $Y_{i}$, что $\operatorname{diam} f\left(Y_{i}\right)<\varepsilon / 2$ и граница $Y_{i}$, обозначаемая $Y_{i}^{\prime}$, не пересекает $Y^{\prime}$ ни при каком $i$.

Пусть $B_{i}$ - шар радиуса $\varepsilon / 2$, содержащий образ $f\left(Y_{i}\right)$. Обозначим через $X_{i}$ пересечение этого шара с $X$. Тогда наша задача будет решена, если для любого $i$ мы построим отображение $f_{i}: Y_{i} \rightarrow B_{i} \backslash X_{i}$, которое совпадает с $f$ на $Y_{i}^{\prime}$. Действительно, в этом случае отображение $f^{\prime}$, совпадающее с $f_{i}$ на $B_{i}$ и с $f$ на дополнении к их объединению, будет непрерывной $\varepsilon$-аппроксимацией, минующей $X$.

Итак, мы имеем задачу продолжения. Но такая задача в данном случае разрешима, потому что лемма об экстенсивности позволяет утверждать, что $Y_{i} \tau B_{i} \backslash X_{i}$.

СТАТИЧЕСКАЯ ВЕРХНЯЯ ОЦЕНКА. Пусть $Y-$ сигма-компакт размерности $<n$, лежаший в $\mathbb{R}^{n}(n>3)$, и $X-$ компакт размерности $<n-1$. Тогда для почти всех отображсений $f: X \rightarrow \mathbb{R}^{n}$ имеем: $\operatorname{Dim}(f(X) \cap Y) \leqslant(\operatorname{Dim} X[+] \operatorname{Dim} Y)\langle-\rangle n$.

ДокАЗАТЕЛЬСтво. При доказательстве этой теоремы мы следуем А.Н. Дранишникову [34]. Пусть $D=(\operatorname{Dim} X[+] \operatorname{Dim} Y)\langle-\rangle n$ и $D^{\prime}=\operatorname{Dim} Y\langle-\rangle(D+1)$, тогда $\operatorname{Dim} Y \leqslant D^{\prime}\langle+\rangle D+1$. Пусть $M$ и $N$ - абелевы комплексы, для которых $\operatorname{Cin} M=D^{\prime}$ и $\operatorname{Cin} N=D$. Тогда $\operatorname{Cin} M * N=\operatorname{Cin} M\langle+\rangle \operatorname{Cin} N+1$, и по критерию экстенсивности имеем $Y \tau M * N$. Пусть $Y=Y_{1} \cup Y_{2}$ - разложение сигма-компакта $Y$, поставляемое теоремой Урысона-Дранишникова, так что $Y_{1} \tau M$ и $Y_{2} \tau N$ и, кроме того, $Y_{2}$ можно, в силу уточнения к этой теореме, считать содержащим множество дикости компакта $Y$.

Действительно, если $\operatorname{dim} Y<n-1$, то множество дикости нульмерно и ничто не препятствует его включению в $Y_{2}$. Если же $\operatorname{Dim} Y=n-1$, то множество дикости одномерно и для включения его в $Y_{2}$ необходимо, чтобы $D \geqslant 1$. Последнее вьполнено, если $\operatorname{dim} X>1$. Поэтому теорему в случае $\operatorname{dim} Y=n-1$ и $\operatorname{dim} X=1$ нужно доказывать отдельно. Мы оставляем этот случай читателю.

Заменяя $Y_{1}$ на дополнение к $Y_{2}$, добиваемся того, что $Y_{1}$ имеет тип $F_{\sigma}$ и является ручным. Размерностньй код у любого компакта в $Y_{1}$ не превосходит $D^{\prime}$, и если мы докажем, что $D^{\prime}[+] \operatorname{Dim} X$ не превосходит $n-1$, то в силу критерия пренебрежимости получим $X$-пренебрежимость любого компакта в $Y_{1}$, а стало быть, и всего $Y_{1}$ как суммы счетного числа $X$-пренебрежимых. А поскольку множество отображений ком- 
пакта, минующих множество типа $F_{\sigma}$, имеет тип $G_{\delta}$, то мы и докажем, что оценка на размерность пересечения вьполнена для почти всех отображений. Таким образом, для доказательства теоремы нам остается убедиться в справедливости неравенства $D^{\prime}[+] \operatorname{Dim} X \leqslant n-1$, которое равносильно следуюшему: $D^{\prime} \leqslant(n-1)[-] \operatorname{Dim} X=$ $(n-1)-\operatorname{Dim} X$ (замена минорной разности на двухстороннюю опирается на неравенство $\operatorname{dim} X \leqslant n-2)$. Подставляя значение $D^{\prime}$ в левую часть, получаем неравенство $(\operatorname{Dim} Y\langle-\rangle(D+1)) \leqslant(n-1)-\operatorname{Dim} X$. Положим $x=\operatorname{Dim} X, y=\operatorname{Dim} Y$ и $m=n-1$. Тогда неравенство примет такой вид $y\langle-\rangle(((x[+] y)\langle-\rangle(m+1))+1) \leqslant m-x$. Вычитаемое в левой части в силу транспозиционного неравенства оценивается снизу как $(x[+] y+1)\langle-\rangle(m+1)$, что в силу принципа сокрашения равно $(x[+] y)\langle-\rangle m$. Поэтому левая часть оценивается сверху выражением $y\langle-\rangle((x[+] y)\langle-\rangle m)$, и наше неравенство вытекает из следуюшего: $y\langle-\rangle((x[+] y)\langle-\rangle m) \leqslant m-x$. Перенося вычитаемое из левой части вправо, получаем такое неравенство: $((x[+] y)\langle-\rangle m)\langle+\rangle(m-x) \geqslant y$. Но, опираясь на неравенства транспозиции, ассоциативности и компенсации, можем написать такую цепочку неравенств: $((x[+] y)\langle-\rangle m)\langle+\rangle(m-x) \geqslant((x[+] y)\langle+\rangle(m-x))\langle-\rangle m \geqslant$ $((x\langle+\rangle(m-x))[+] y)\langle-\rangle m \geqslant(m+y)\langle-\rangle m=y$. Что и требовалось доказать.

ДИНАМИЧЕСКАЯ ВЕРХНЯЯ ОЦЕНКА. Если $X$ и $Y$ - компакты размерностей $<n-1, n>3$, тогда для почти всех отображений $f, g: X, Y \rightarrow \mathbb{R}^{n}$ пересечение $f(X) \cap g(Y)$ ручное и справедливо неравенство

$$
\operatorname{Dim}(f(X) \cap g(Y)) \leqslant \operatorname{Dim} X[+] \operatorname{Dim} Y\langle-\rangle n .
$$

ДокАЗАТЕльство. Пусть $D=(\operatorname{Dim} X[+] \operatorname{Dim} Y)\langle-\rangle n$ и $D^{\prime}=(n-1)\langle-\rangle D$, тогда $n \leqslant D^{\prime}\langle+\rangle D+1$. Пусть $M$ и $N$ - абелевы комплексы, для которых $\operatorname{Cin} M=D^{\prime}$ и $\operatorname{Cin} N=D$. Тогда $\operatorname{Cin} M * N=\operatorname{Cin} M\langle+\rangle \operatorname{Cin} N+1$ и по критерию экстенсивности имеем $\mathbb{R}^{n} \tau M * N$.

Пусть $\mathbb{R}^{n}=R_{1} \cup R_{2}$ - разложение пространства $Y$, поставляемое теоремой Урысона-Дранишникова, так что $R_{1} \tau M$ и $R_{2} \tau N$.

Заменяя $R_{1}$ на дополнение к $R_{2}$, добиваемся того, что $R_{1}$ имеет тип $F_{\sigma}$. Пусть $Z \subset R_{1}$ - произвольньй компакт. Тогда $\operatorname{Dim} Z \leqslant D^{\prime}<n-1$. Поэтому, в силу только что доказанной теоремы, для почти всех отображений $f: X \rightarrow \mathbb{R}^{n}$ размерность пересечения оценивается сверху как $\operatorname{Dim} X[+] \operatorname{Dim} Z\langle-\rangle n$. Кроме того, почти все отображения минуют множество дикости компакта $Z$ (ибо последнее является ручным). Поэтому для почти всех отображений пересечение $f(X) \cap Z$ является ручным. Поэтому можно воспользоваться критерием Дранишникова, чтобы доказать его $Y$-пренебрежимость. Для этого нужно проверить справедливость неравенства $\operatorname{dim}(f(X) \cap Z) \times Y<n$. То есть достаточно доказать следующее неравенство: $((\operatorname{Dim} X[+] \operatorname{Dim} Z)\langle->n)[+] \operatorname{Dim} Y \leqslant n-1$. Подставляя вместо $\operatorname{Dim} Z$ его верхнюю оценку, получаем:

$$
((\operatorname{Dim} X[+]((n-1)-(\operatorname{Dim} X[+] \operatorname{Dim} Y)\langle->n))\langle->n)[+] \operatorname{Dim} Y \leqslant n-1 .
$$

Размерностные коды компактов далее, ради краткости, будем обозначать соответствующими малыми буквами. Перенося $\operatorname{Dim} Y=y$ вправо, в соответствии с принятыми сокрашениями получаем следующее эквивалентное неравенство:

$$
(x[+]((n-1)-(x[+] y)\langle->n))\langle->n \leqslant(n-1)[-] y .
$$


Поскольку $y \leqslant n-2$, разность справа - двухсторонняя. Поэтому, перенося $n$ из левой части вправо, получаем в правой части $((n-1)-y)\langle+\rangle n$, что в силу транспозиционного неравенства не уступает $(2 n-1)-y$. Поэтому нужное нам неравенство вытекает из следуюшего: $(x[+]((n-1)-(x[+] y)\langle->n)) \leqslant(2 n-1)-y$. В силу принципа сокрашения имеем $(n-1)-(x[+] y)\langle-\rangle n=(2 n-1)-(((x[+] y)\langle-\rangle n)+n), \mathrm{a}$ так как $((x[+] y)\langle-\rangle n)+n \geqslant(x[+] y)$, то левая часть оценивается сверху величиной $x[+]((2 n-1)-(x[+] y))$. Поэтому нужное нам неравенство вытекает из следующего: $x[+]((2 n-1)-(x[+] y)) \leqslant(2 n-1)-y$. Так как разность справа двухсторонняя, то, считая ее минорной и перенося $y$ влево, получаем, что последнее неравенство эквивалентно следуюшему: $(x[+] y)[+]((2 n-1)-(x[+] y)) \leqslant 2 n-1$. А это неравенство справедливо в силу принципов компенсации, поскольку $x+y \leqslant 2 n-4$ и разность слева, будучи двухсторонней, может рассматриваться как минорная.

Итак, пересечение $f(X) \cap Z$ почти для всех $f$ является $Y$-пренебрежимым. Поэтому для почти всех пар отображений $f, g: X, Y \rightarrow \mathbb{R}^{n}$ имеем: $f(X) \cap f(Y)$ не пересекается ни с каким компактом в $R_{1}$, а значит, и со всем $R_{1}$, ибо оно является счетным объединением компактов. Откуда вытекает, что это пересечение почти всегда содержится в $R_{2}$, и потому имеет размерность $\leqslant D$, что и требовалось доказать.

\section{Верхние оценки кратных пересечений}

КРИТЕРИЙ ПРЕНЕБРЕЖИМОСТИ ПОЛИКОМПАКТОМ. Пусть $X \subset \mathbb{R}^{n}(n>3)$ является компактом размерности $<n$. Тогда $X$ является пренебрежимылм поликомпактом У размерности $<n-1$ и длинь $m>1$ в том и только том случае, $\kappa о г д а \operatorname{dim} X \times \prod \mathscr{Y}<n m$.

ДокАЗАТЕльство. Если $\operatorname{dim} X \times \prod \mathscr{Y} \geqslant n m$, то непренебрежимость $X$ поликомпактом $\mathscr{Y}$ непосредственно вытекает из теоремы о непренебрежимости.

Пусть теперь $\operatorname{dim} X \times \prod \mathscr{Y}<n m$. Докажем в этом случае $\mathscr{Y}$-пренебрежимость компакта $X$. Доказательство ведем индукцией по длине поликомпакта $m$. Пусть $m=2$. Тогда множество дикости компакта $X$ ручное и не более чем одномерное и потому является $Y_{1}$-пренебрежимьм в силу критерия Дранишникова, так как $\operatorname{dim} Y_{1} \leqslant n-2$. Значит, для почти всех отображений из $Y_{1}$ в $\mathbb{R}^{n}$ пересечение их с $X$ является ручным. Далее, из доказанной выше теоремы следует, что для почти всех отображений из $Y_{1}$ в $\mathbb{R}^{n}$ вьполнено неравенство $\operatorname{Dim} f\left(Y_{1}\right) \cap X \leqslant$ $\operatorname{Dim} X \times Y_{1}\langle-\rangle n$, из которого, в частности, следует, что размерность пересечения $f$ с $X$ не превосходит $n-3$. Поэтому к этому пересечению применим критерий пренебрежимости Дранишникова, и для доказательства $Y_{2}$-пренебрежимости этого пересечения, а с ним и доказательства $\mathscr{Y}$-пренебрежимости компакта $X$ достаточно проверить неравенство $\left(\operatorname{Dim} X \times Y_{1}\langle-\rangle n\right)[+] \operatorname{Dim} Y_{2} \leqslant n-1$. Перенося вправо сначала $\operatorname{Dim} Y_{2}$, а потом $n$, получаем, что это неравенство равносильно следуюшему: $\operatorname{Dim} X \times Y_{1} \leqslant n\langle+\rangle\left((n-1)[-] \operatorname{Dim} Y_{2}\right)$. Но правая часть равна $(2 n-1)-\operatorname{Dim} Y_{2}$, поскольку $\operatorname{Dim} Y_{2} \leqslant n-2$, и потому разность справа - двухсторонняя. Перенося теперь $\operatorname{Dim} Y_{2}$ влево, получаем неравенство $\operatorname{Dim} X \times Y_{1} \times Y_{2} \leqslant 2 n-1$, равносильное тому условию на размерность произведения, которое нам дано. Итак, случай $m=2$ разобран.

Пусть наше утверждение доказано для поликомпактов длины $<m$. Так же как и в случае $m=2$, заметим, что для почти всех отображений из $Y_{1}$ в $\mathbb{R}^{n}$ пересечение их 
образа $X^{\prime}=X \cap g\left(Y_{1}\right)$ имеет размерностньй код $\operatorname{Dim} X^{\prime} \leqslant \operatorname{Dim} X[+] \operatorname{Dim} Y_{1}\langle-\rangle n$.

Докажем, что компакт $X^{\prime}$ с таким кодом является пренебрежимым поликомпактом $\mathscr{Y}^{\prime}=\left\{Y_{2}, \ldots, Y_{m}\right\}$. Отсюда немедленно вытекает $\mathscr{Y}$-пренебрежимость компакта $X$.

Поскольку длина $\mathscr{Y}^{\prime}$ не меньше двух и меньше $m$, для него критерий пренебрежимости справедлив в силу предположения индукции. Воспользуемся этим критерием. Согласно этому критерию нам нужно доказать такое неравенство: $\operatorname{dim}\left(X^{\prime} \times \prod \mathscr{Y}^{\prime}\right)<$ $n(m-1)$.

Подставляя вместо $\operatorname{Dim} X^{\prime}$ его верхнюю оценку, сводим задачу к доказательству следующего неравенства:

$$
\left(\operatorname{Dim} X \times Y_{1}\langle->n)[+] \operatorname{Dim} \prod \mathscr{Y}^{\prime}<m n-n\right.
$$

Доказательство этого неравенства получается из рассуждения для $m=2$, если вместо $\operatorname{Dim} Y_{2}$ подставить $\operatorname{Dim} \prod \mathscr{Y}^{\prime}$.

ДИНАМИЧЕСКАЯ ВЕРХНЯЯ ОЦЕНКА. Пусть $\mathscr{X}-$ поликомпакт длины $m \geqslant 2$ и размерности $<n-1$. Тогда всякое отображсние из $\mathscr{X}$ в $\mathbb{R}^{n}$ можнно аппроксимировать таким, размерностный код пересеченного образа которого не превосходит $\operatorname{Dim} \prod \mathscr{X}\langle-\rangle(m n-n)$.

ДокАЗАТЕльство. Доказательство проводится индукцией по $m$. Если $m=2$, то соответствуюшая формула совпадает с полученной ранее для пары отображений. Пусть она доказана для $m=k$. В таком случае произвольный набор отображений компактов $X_{1}, \ldots, X_{k+1}$ апроксимируется набором, для которого пересечение образов всех, за исключением последнего, компактов оцениваются сверху величиной $D=\operatorname{Dim} \prod_{i=0}^{k} X_{i}\langle-\rangle(k n-n)$ и которое к тому же можно предполагать ручным. Обозначим это пересечение через $Y$ и оценим сверху размерность пересечения $X_{k+1}$ с $Y$ согласно статической верхней оценке. Получим: $\left(D[+] \operatorname{Dim} X_{k+1}\right)\langle-\rangle n$. Пусть $y=\operatorname{Dim} \prod_{i=1}^{k} X_{i}, y^{\prime}=\operatorname{Dim} X_{k+1}$. Таким образом, нам достаточно доказать справедливость следуюшего неравенства: $\left((y\langle-\rangle(k-1) n)[+] y^{\prime}\right)\langle-\rangle n \leqslant\left(y+y^{\prime}\right)\langle-\rangle k n$. Перенося вправо $n$, получаем, что нам достаточно доказать такое неравенство: $y\langle-\rangle(k-1) n \leqslant\left(\left(\left(y[+] y^{\prime}\right)\langle-\rangle n k\right)+n\right)[-] y^{\prime}$. По формуле транспозиции правая часть оценивается снизу как $\left(\left(y[+] y^{\prime}\right)\langle->n k)[+]\left(n[-] y^{\prime}\right)\right.$, и вторичное применение транспозиции с учетом неравенства $y^{\prime} \leqslant n-1$ дает такую нижнюю оценку правой части: $\quad\left(\left(y[+] y^{\prime}\right)[+]\left(n-y^{\prime}\right)\right)\langle-\rangle n k=\left(y[+]\left(y^{\prime}[+]\left(n-y^{\prime}\right)\right)\right)\langle-\rangle n k \geqslant(y[+] n)\langle-\rangle n k=$ $y\langle-\rangle(k-1) n$. Что и требовалось доказать.

КРИТЕРИЙ СУЩЕСТВОВАНИЯ УСТОЙЧИВЫХ ПЕРЕСЕЧЕНИЙ. Пусть $\mathscr{X}-$ nоликомпакт размерности $<n-1$, длины $m>2$, тогда он имеет устойчивье пересечения в $\mathbb{R}^{n}$ в том и только том случае, когда $\operatorname{dim} \prod \mathscr{X} \geqslant m n-n$.

ДокАЗАТЕльство. Достаточность условия на размерность произведения вытекает из теоремы об устойчивом пересечении.

В силу формулы пересечения отображений почти все отображения поликомпакта $X_{1}, X_{2}$ имеют ручной пересеченный образ с размерностным кодом, не превосходящим даваемого формулой пересечения. Пересечение образов $X_{1}$ и $X_{2}$ обозначаем $Y$. Докажем, что $Y$ является пренебрежимьм поликомпактом $X_{3}, \ldots, X_{m}$, опираясь на доказанный вьше критерий. Итак, нам известно, что 
$\operatorname{Dim} Y \leqslant\left(\operatorname{Dim} X_{1}[+] \operatorname{Dim} X_{2}\right)\left\langle->n\right.$. Обозначим $\operatorname{Dim} X_{1} \times X_{2}=x$ и $\operatorname{Dim} \prod_{i=3}^{m} X_{i}=z$. Тогда $x[+] z \leqslant(m-1) n$, а нам нужно доказать, что $(x\langle-\rangle n)[+] z \leqslant(m-2) n$. Перенося в последнем неравенстве слева направо $z$ и $n$, получаем эквивалентное неравенство $x \leqslant((m-2) n[-] z)+n$. Но $z<(m-2) n$, поэтому разность справа двухсторонняя, и, трактуя ее как мажорную, можем на основании транспозиционного неравенства заключить: $((m-2) n[-] z)+n \geqslant(m-1) n-z$. Поэтому нужное нам неравенство следует из неравенства $x \leqslant(m-1) n-z$, равносильного условию $x[+] z \leqslant(m-1) n$. Что и требовалось доказать.

СТАТИЧЕСКАЯ ВЕРХНЯЯ ОЦЕНКА. Пусть $X$ - является сигма-компактным подмножеством в $\mathbb{R}^{n}$ размерности $\operatorname{dim} X<n$, пусть $n>3$, и пусть дан поликомпакт УУ длинь $m \geqslant 1$ и размерности $<n-1$. Тогда для почти всех отображений $F: \mathscr{Y} \rightarrow \mathbb{R}^{n}$ справедливо неравенство

$$
\operatorname{Dim}_{X} F^{\cap}(\mathscr{Y}) \leqslant \operatorname{Dim}\left(X \times \prod \mathscr{Y}\right)\langle-\rangle n m .
$$

ДокАЗАТЕЛЬСтво. Доказательство ведем индукцией по $m$. В соответствии со статической теоремой для пар, почти всякое отображение из $Y_{1}$ в $\mathbb{R}^{n}$ имеет ручное пересечение образа с $X$, обозначаемое $Y$, размерности $\operatorname{Dim} Y \leqslant \operatorname{dim} X \times Y_{1}\langle-\rangle n \leqslant n-3$. Пусть $\mathscr{Y}^{\prime}=Y_{2}, \ldots, Y_{m}$. Тогда по предположению индукции получается, что для почти всех отображений из $\mathscr{Y}^{\prime}$ в $\mathbb{R}^{n}$ размерность пересечения с $Y$ оценивается сверху величиной $((D\langle->n)[+] C)\langle->k n$, где через $C$ обозначен размерностньй код произведения компактов из $\mathscr{Y}^{\prime}$, через $D$ - размерностный код произведения $X \times Y_{1}$, а $k$ равняется длине $\mathscr{Y}^{\prime}$. Поэтому теорема будет доказана, если мы докажем неравенство $((D\langle-\rangle n)[+] C)\langle-\rangle k n \leqslant(D[+] C)\langle-\rangle(k+1) n$, подобное тому, которое мы доказьвали при вьводе формулы кратного пересечения.

\section{$\S$ 9. Нижние оценки размерности пересечения}

Вьше мы, исходя из размерностей компактов, оценивали сверху размерность их типичных пересечений с поликомпактами. Теперь мы займемся обратной задачей. Обратную задача заключается в получении размерностных оценок компакта через типичные размерности его пересечений. Задачи такого типа мы будем назьвать томографическими.

ТОМОГРАФИЧЕСКАЯ ВЕРХНЯЯ ОЦЕНКА. Пусть $\mathscr{X}$ - поликомпакт размерности $<n$, длины $m$ и $Y$ - сигма-компактное подмножество в $\mathbb{R}^{n}$ (где $n>3$ ) такие, что $\operatorname{dim} Y \times \prod \mathscr{X} \geqslant$ тп. Если для почти всех отображений из $\mathfrak{F}\left(\mathscr{X}, \mathbb{R}^{n}\right)$ выполнено неравенство $\operatorname{Dim} F^{\cap}(\mathscr{X}) \cap Y \leqslant C$, mо $\operatorname{Dim} Y \leqslant C\langle+\rangle\left(m n-\operatorname{Dim} \prod \mathscr{X}\right)$.

ДокАЗАТЕЛЬСТВо. Зафиксируем всюду плотную в пространстве отображений $\mathfrak{F}\left(\mathscr{X}, \mathbb{R}^{n}\right)$ последовательность $F_{i}$, такую что $\operatorname{Dim}\left(F_{i}^{\bigcap}(\mathscr{X}) \cap Y\right) \leqslant C$ для любого $i$. Обозначим объединение этих пересечений через $Y_{1}$. Тогда $\operatorname{Dim} Y_{1} \leqslant C$. Заключим $Y_{1}$ в $G_{\delta}$-множество $Y_{1}^{\delta}$, имеюшее тот же размерностный код (применяем теорему Ольшевского). Тогда дополнение $Y_{2}=Y Y_{1}^{\delta}$ является объединением счетного числа компактов $Y_{2}^{i}$, которые $\mathscr{X}$-пренебрежимы. Тогда согласно теореме о непренебрежимости компакты $Y_{2}^{i}$ не имеют устойчивых пересечений с $\mathscr{X}$, и по теореме об устойчивом пересечении мы заключаем, что $\operatorname{dim} X \times Y_{2}^{i}<n$. Поэтому код $\operatorname{Dim} Y_{2}$ 
не превосходит $(m n-1)[-] \operatorname{Dim} \prod \mathscr{X}$. В таком случае для $Y$ код оценивается сверху согласно неравенству Урысона-Дыдака величиной $\left((m n-1)[-] \operatorname{Dim} \prod \mathscr{X}\right)\langle+\rangle C+1$. Поскольку $\operatorname{Dim} \mathscr{X}<n$, постольку минорная разность в этой формуле определена и возможно сокращение единиц. В результате верхняя оценка для $\operatorname{Dim} Y$ приобретает требуемый вид.

ЛЕММА О ТИПИЧНОМ ПЕРЕСЕЧЕНИИ. Пусть даны компакт $X \subset \mathbb{R}^{n}$ u поликомпакт У, тогда существует такая кодировка D, что:

(1) $\operatorname{Dim}\left(F^{\cap}(\mathscr{Y}) \cap X\right) \leqslant D$ для почти всех $F \in \mathfrak{F}\left(\mathscr{Y}, \mathbb{R}^{n}\right)$;

(2) первое условие не вьполняется ни для какой $C^{\prime}<C$.

ДоКАЗАТЕЛЬСТВо. Для кодировки $C$ положим $\mathfrak{F}_{C}=\left\{F \mid \operatorname{Dim}\left(F^{\cap}(\mathscr{Y}) \cap X\right) \leqslant C\right\}$. Пусть $\Delta=\left\{C \in \mathfrak{C D} \mid \mathfrak{F}_{C}\right.$ всюду плотно $\}$. Тогда, ввиду счетной тесноты кодировок, найдется такая последовательность кодировок $\left\{D_{i}\right\}$, нижняя грань которой, обозначаемая $D$, совпадает с нижней гранью $\Delta$. При любом $C \in \Delta$ множество $\mathfrak{F}_{C}$ полно, как следует из поликомпактной версии леммы о полноте из $\S 7$. Тогда и $\mathfrak{F}_{D}$ всюду плотно и имеет тип $G_{\delta}$. Поэтому $D$ удовлетворяет первому условию. И $D$ удовлетворяет второму условию ввиду того, что $D=\inf \Delta$.

Кодировка $D$, о которой идет речь в доказанной лемме, называется типичной размерностью пересечения компакта $X$ и поликомпакта $\mathscr{Y}$ и обозначается $\operatorname{Dim}_{\mathscr{Y}}^{\bigcap} X$.

C помошью этого понятия мы можем представить результат предыдущей теоремы как нижнюю оценку для типичной размерности пересечения.

СлЕДСТВИЕ. При условиях теоремы о статической томографии справедливо неравенство:

$$
\operatorname{Dim}_{\mathscr{X}}^{\cap} Y \geqslant \operatorname{Dim}\left(Y \times \prod \mathscr{X}\right)\langle-\rangle m n
$$

Сопоставляя этот результат со статической теоремой о верхней оценке, получаем следуюший результат:

ФОРМУЛА ТИПИЧНОГО ПЕРЕСЕЧЕНИЯ. Пусть $Y \subset \mathbb{R}^{n}(n>3)$ является компактом размерности $<n, \mathscr{X}-$ поликомпактом размерности $\leqslant n-2$ и длинь $m$, u nусть $\operatorname{Dim}\left(Y \times \prod \mathscr{X}\right) \geqslant m n$. Tогда $\operatorname{Dim}_{\mathscr{X}}^{\cap} Y=C\langle+\rangle\left(m n-\operatorname{Dim} \prod \mathscr{X}\right)$.

Развитая теория позволяет также получить оценки для коэффициентных когомологических размерностей пересечений.

ЛЕММА О КОМПЛЕКСЕ ЭЙЛЕНБЕРГА-МАКЛЕЙНА. $\|\operatorname{Cin} K(G, n)\|_{G}=n$.

ДокАЗАТЕЛьСтвО. Действительно, комплекс Эйленберга-Маклейна характеризуется тем, что $X \tau K(G, n)$ равносильно $\operatorname{dim}_{G} X \leqslant n$. В силу критерия экстенсивности Дранишникова получаем отсюда, что $\operatorname{Cin} K(G, n)=\sup \left\{D \mid\|D\|_{G} \leqslant n\right\}$. Так как существуют коды, имеющие верхнюю $G$-норму, равную $n$, то из монотонности $G$-нормы получаем $\|\operatorname{Cin} K(G, n)\|_{G} \geqslant n$. C другой стороны, компакт $X$ размерности $\operatorname{Dim} X=\operatorname{Cin} K(G, n)$ экстенсивен к $K(G, n)$ и потому имеет $G$-размерность $\leqslant n$. Откуда и следует обратное неравенство. 
НИжНЯя КОЭФФИЦИЕНТНАЯ ОЦЕНКА. Пусть $G$ - какая-то абелева әруппа, $\mathscr{X}-$ поликомпакт размерности $<n$, длины $m u X_{0} \subset \mathbb{R}^{n}$ - сигма-компактное множество. Тогда если $\operatorname{dim}\left(X_{0} \times \prod \mathscr{X}\right) \geqslant m n$, то непустую внутренность имеет множество отображсений $F$, удовлетворяющих неравенству

$$
\operatorname{dim}_{G} X_{0} \cap F^{\cap}(\mathscr{X}) \geqslant \operatorname{dim}_{G}\left(X_{0} \times \prod \mathscr{X}\right)-m n .
$$

ДокАЗАТЕЛЬСТвО. Пусть $N=\operatorname{dim}_{G}\left(X_{0} \times \prod \mathscr{X}\right)-m n$. Предположение противного приводит к утверждению, что всюду плотно в $\mathfrak{F}\left(\mathscr{X}, \mathbb{R}^{n}\right)$ множество отображений $F$, для которых $\operatorname{dim}_{G} X_{0} \cap F^{\cap}(\mathscr{X}) \leqslant N-1$. Обозначим через $C$ связностньй код комплекса $K(G, N-1)$. Тогда в силу критерия экстенсивности получим, что $\operatorname{Dim} X_{0} \cap F^{\cap}(\mathscr{X}) \leqslant C$ для всюду плотного множества отображений. Откуда в силу полученной вьше нижней оценки типичной размерности пересечения получим неравенство $C \geqslant \operatorname{Dim}\left(X_{0} \times \prod \mathscr{X}\right)\langle-\rangle m n$. Откуда $\|C\|_{G} \geqslant\left\|\operatorname{Dim}\left(X_{0} \times \prod \mathscr{X}\right)\langle-\rangle m n\right\|_{G}$. Но $\|C\|_{G}=N-1$ согласно лемме о комплексе Эйленберга-Маклейна. Полученное противоречие доказывает теорему.

\section{Теорема об устойчивой нижней оценке}

Будем говорить, что компакт $X \subset \mathbb{R}^{n}$ динамически пренебрежим компактом $Y$, если для любого отображения $f: Y \rightarrow \mathbb{R}^{n}$ и любого $\varepsilon>0$ найдутся такие $\varepsilon$-сдвиг $g$ компакта $X$ и $\varepsilon$-близкое к $f$ отображение $f^{\prime}$ компакта $Y$, что $f^{\prime}(Y) \cap g(X)=\varnothing$. Другими словами, если тождественное вложение компакта $X$ неустойчиво пересекает класс отображений $\mathfrak{F}\left(Y, \mathbb{R}^{n}\right)$.

АДДИЦИОННАя ЛЕмМА. Если два компакта $X_{1}, X_{2} \subset \mathbb{R}^{n}$ являются динамически пренебрежимыми компактом $Y$, а их пересечение является $Y$-пренебрежимым, то их обвединение будет динамически $Y$-пренебрежимым.

ДокАЗАТЕЛЬство. Пусть даны произвольное отображение $f: Y \rightarrow \mathbb{R}^{n}$ и положительное $\varepsilon$. В силу условия пренебрежимости пересечения можем, не нарушая обшности рассуждений, предполагать, что $f(Y) \cap X_{1} \cap X_{2}=\varnothing$. Пусть $\delta$ столь мало, что $f(Y) \cap O_{\delta} X_{1} \cap O_{\delta} X_{2}=\varnothing$. Пусть $\gamma=\frac{1}{9} \min \{\delta, \varepsilon\}$. В силу динамической пренебрежимости $X_{i}(i=1,2)$ найдутся такое $\gamma$-близкое к $f$ отображение $f_{i}$ и такой $\gamma$-сдвиг $g_{i}: X_{i} \rightarrow \mathbb{R}^{n}$, что $g_{i}\left(X_{i}\right) \cap f_{i}(Y)=\varnothing$.

Пусть $Y_{i}=\left\{y \in Y \mid \operatorname{dist}\left(f(y), X_{i}\right)<4 \gamma\right\}(i=1,2)$ и $Y_{0}=Y \backslash\left(Y_{1} \cup Y_{2}\right)$. Множества $Y_{1}$ и $Y_{2}$ не пересекаются, ибо $4 \gamma<\delta / 2$. Зафиксируем функцию Урысона $\beta(y)$ так, что $\beta\left(Y_{1}\right)=0$ и $\beta\left(Y_{2}\right)=1$.

Пусть также $X_{0}$ - это открытая $\gamma$-окрестность пересечения $X_{1} \cap X_{2}$ в $X$ и $\alpha: X \rightarrow$ $[0,1]$ - функция Урысона, принимаюшая значение 0 на $X_{1}$ и 1 на $X_{2}$.

Произвольным образом продолжая отображения $g_{1}$ и $g_{2}$ до $\gamma$-сдвигов всего $X$, определим теперь отображения $f^{\prime}: Y \rightarrow \mathbb{R}^{n}$ и $g: X \rightarrow \mathbb{R}^{n}$ следуюшими формулами:

$$
f^{\prime}(y)=(1-\beta(y)) f_{1}(y)+\beta(y) f_{2}(y), \quad g(x)=(1-\alpha(x)) g_{1}(x)+\alpha(x) g_{2}(x) .
$$

Тогда $f^{\prime}$ будет $\gamma$-близко к $f$, а $g$ будет $\gamma$-сдвигом. Убедимся, что $g(X) \cap f^{\prime}(Y)=\varnothing$. Если $y \in Y_{1}$, то $f^{\prime}(y)=f_{1}(y)$ не принадлежит $g\left(X_{1} \backslash X_{0}\right)=g_{1}\left(X_{1} \backslash X_{0}\right)$ в силу условия $g_{1}\left(X_{1}\right) \cap f_{1}(Y)=\varnothing$. Далее, $X_{0} \cup X_{2}$ содержится в $\gamma$-окрестности $X_{2}$, поэтому 
образ этого множества при $g$ содержится в $2 \gamma$-окрестности компакта $X_{2}$. С другой стороны, $\operatorname{dist}\left(f^{\prime}(y), f(y)\right)<\gamma$, поэтому принадлежность $g(y)$ образу $f^{\prime}\left(X_{0} \cup X_{2}\right)$ влечет неравенство $\operatorname{dist}\left(f(y), X_{2}\right)<3 \gamma$, что несовместимо с принадлежностью $y \in Y_{1}$. Итак, $f^{\prime}\left(Y_{1}\right) \cap g(X)=\varnothing$. Аналогично $f^{\prime}\left(Y_{2}\right) \cap g(X)=\varnothing$. Если же $y \in Y_{0}$, то $\operatorname{dist}(f(y), X)>4 \gamma$, поэтому $\operatorname{dist}\left(f^{\prime}(y), X\right)>3 \gamma$, откуда $\operatorname{dist}\left(f^{\prime}(y), g(X)\right)>2 \gamma$. То есть $f^{\prime}(y) \notin g(X)$. Что и завершает доказательство.

ЛЕМма О ГИПЕРПЛОСКОМ КОМПАКТЕ. Всякий компакт $X$ коразмерности $>2$, лежащий в гиперплоскости, ручной.

ДокАЗАТЕльство. Считаем $H$ линейным подпространством в $\mathbb{R}^{n}$. В силу леммы об одномерном пересечении с диском для почти всех отображений диска $D$ прообраз $H$ одномерен. Пусть $f: D \rightarrow \mathbb{R}^{n}$ - одно из таких отображений. В силу теоремы Франкля при любом $\varepsilon>0$ найдется $\varepsilon$-близкое к $f$ отображение $g: f^{-1}(f(D) \cap H) \rightarrow H$, образ которого не пересекает $X$. Пусть $d f: D \rightarrow H$ - отображение, по модулю не превосходящее $\varepsilon$ и продолжающее отображение $f-g$ с $f^{-1}(f(D) \cap H)$ на все $D$. Тогда $f+d f$ будет отображением, $\varepsilon$-близким к $f$ и не пересекаюшим $X$.

Компактное множество $X \subset \mathbb{R}^{n}$ назьвается клеточно-разделеннылм, если для любого $\varepsilon>0$ существует конечное множество дизъюнктных $n$-клеток (т.е. компактов гомеоморфных $n$-шару) диаметров, меншших $\varepsilon$, внутренности которых покрывают $X$.

КРИТЕРИЙ МАК-МИЛЛАНА [45]. Нульмерное подмножество в $\mathbb{R}^{n}$ является клеточно-разделенным в том и только том случае, когда оно ручное.

Критерий Мак-Миллана имеет сравнительно элементарное доказательство при $n>4$ и именно при этом ограничении доказан Мак-Милланом. При $n=4$ он вытекает из результатов $Ф$ ридмана о четырехмерной гипотезе Пуанкаре. В размерности 3 его нетрудно вьвести из леммы Дена.

ЛЕмма о $\varepsilon$-ИзОтоПИИ. Пусть дан $X$ - ручной нульмерный компакт, а $Y$ некоторое замкнутое нигде не плотное подмножество $\mathbb{R}^{n}$. Тогда при любом $\varepsilon>0$ найдется $\varepsilon$-изотопия, сдвигающая $X$ с $Y$ и неподвижная за пределами в-окрестности $X$.

ДокАЗАТЕЛЬСТво. Покроем $X$ дизъюнктными клетками $C_{i}$ диаметров $<\varepsilon$. Во внутренности каждой клетки выберем точку $x_{i}$, не лежащую в $Y$. Введем на $C_{i}$ координаты так, чтобы она представляла собой единичньй шар с центром в $x_{i}$. Тогда для некоторого $r>0$ шар радиуса $r$ не пересекает $Y$ и для некоторого $R<1$ шар радиуса $R$ содержит $X \cap C_{i}$. Если теперь обозначить через $l(t)$ гомеоморфизм отрезка $[0,1]$, для которого $l(1)=1$ и $l(R)=r$, то изотопия в сферических координатах $\varphi, \rho$, задаваемая формулой $(\varphi, \rho) \rightarrow(\varphi,(1-t) \rho+t l(\rho))$ неподвижна на границе клетки и сдвигает $X \cap C_{i}$ с $Y$. Совокупность таких изотопий задает неподвижную за пределами объединения клеток искомую изотопию пространства.

ЛЕМма О НУЛЬМЕРНОм $F_{\sigma}$. Пусть дана последовательность нульмерных компактов $X_{i}$, тогда существует последовательность дизбюнктных компактов $c$ диаметрами, стремящимися к нулю, и таким же обвединением, как первая. 
ДокаЗАТЕльство. Пусть $X_{i}^{\prime}=X_{i} \backslash \bigcup_{j<i} X_{j}$. Тогда $X_{i}^{\prime}$ является дизъюнктной последовательностью из локально-компактных нульмерных множеств. Каждое $X_{i}^{\prime}$ можно представить в виде объединения растущей последовательности дизъюнкт-

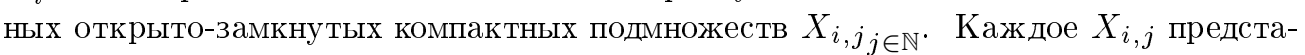
вим в виде объединения конечного числа дизъюнктных компактов $X_{i, j, k}$ диаметров $<1 /(i+j)$. Тогда совокупность всех $X_{i, j, k}$ является искомой последовательностью.

Следующая лемма для ручных $X$ была доказана в [19].

ЛЕММА О ГИПЕРПЛОСКОМ СЕЧЕНИИ. Для всякого компакта $X$, лежсащего $\boldsymbol{\theta}$ $\mathbb{R}^{n}$, и гиперплоскости $H$ при любом $\varepsilon>0$ найдется гомеоморфизм $h: \mathbb{R}^{n} \rightarrow \mathbb{R}^{n}$,

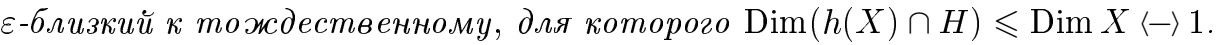

ДокаЗАТЕЛЬСтво. Пусть $D=\operatorname{Dim} X\langle-\rangle$. Тогда $\operatorname{Dim} X \leqslant D+0+1$, и по критерию экстенсивности имеем $X \tau \Sigma L$, где $\operatorname{Cin} L=D$. По уточненной теореме о разложе-

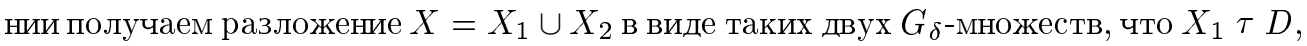
$\operatorname{dim} X_{2}=0$ и $X_{1}$ содержит множество дикости компакта $X$. Тогда дополнение к $X_{1}$, обозначаемое $Y$, является объединением счетного числа ручных нульмерных компактов. И лемма будет доказана, если мы построим малую изотопию пространства $\mathbb{R}^{n}$, сдвигающую $Y$ с $H$. На основании леммы о $F_{\sigma}$-множестве представим $Y$ в виде объединения последовательности $Y_{i}$ непересекаюшихся компактов со стремяшимися к нулю диаметрами. Пространство гомеоморфизмов $\mathbb{R}^{n}$ топологически полно, поэтому на нем существует полная метрика $\rho$. Приняв это, строим $\rho$-последовательность Коши гомеоморфизмов $\left\{h_{i}\right\}$, предел которой и даст нужньй гомеоморфизм, сдвигаюший $Y$ с $H$. На первом шаге мы построим гомеоморфизм $h_{1}$, сдвигаюший $Y_{1}$ с $H$, находяшийся на не более чем единичном $\rho$-расстоянии от тождественного и сдвигающий точки меньше чем на $\varepsilon / 2$. На $k$-м шаге мы строим гомеоморфизм $h_{k}$, неподвижный на всех $X_{i}$ при $i<k$, сдвигаюший точки меньше чем на $\varepsilon / 2^{k}$ и такой, что $\rho\left(h_{k}, h_{k-1}\right)<1 / 2^{k}$. Все это можно сделать на основании леммы о $\varepsilon$-изотопии. Тогда предел последовательности $h_{k}$ будет гомеоморфизмом ввиду полноты метрики $\rho$, будет $\varepsilon$-сдвигом и будет сдвигать объединение $X_{i}$ с $H$. Что и требовалось доказать.

ЛЕММА О ДИНАМИЧЕСКОЙ ПРЕНЕБРЕЖИМОСТИ. РУчной динамически $Y$-npeнебрежимый компакт $X$ коразмерности $>2$ является $Y$-пренебрежимым.

ДокАЗАТЕльство. Доказательство будем вести индукцией по размерности $X$. Если $\operatorname{dim} X=0$ и $X$ является динамически $Y$-пренебрежимым, то $\operatorname{dim} Y<n$. Действительно, в противном случае найдется существенное, в смысле Александрова, отображение $f: Y \rightarrow \mathbb{R}^{n}$ на шар, содержаший $X$ в своей внутренности. Тогда всякое достаточно близкое отображение к $f$ имеет образ, содержащий некоторую $\varepsilon$-окрестность компакта $X$, и потому пересекает образы $X$ при $\varepsilon$-сдвигах. А раз $\operatorname{dim} Y<n$, то $\operatorname{dim} X \times Y<n$ и $X$ является $Y$-пренебрежимым в силу критерия Дранишникова.

Пусть для компактов размерности $<k$ утверждение доказано. Рассмотрим динамически $Y$-пренебрежимьй компакт $X$ размерности $k$. Докажем, что $X$ с $Y$ в $\mathbb{R}^{n}$ не имеют устойчивых пересечений. Пусть дано какое-то отображение $f: X \rightarrow \mathbb{R}^{n}$ и положительное $\varepsilon$. Зафиксируем столь мелкую триангулящию на $\mathbb{R}^{n}$, чтобы линеаризация продолжения $f$ на некоторую подиэдральную окрестность $O X$ компакта $X$ была $\varepsilon$-близка к $f$. Далее, эту линеаризацию $l$ мы, как в аппроксимационной лемме, можем считать однозначной на всех симплексах триангулящии. Саму триангулящию 
мы в силу леммы о гиперплоском сечении можем, не нарушая обшности, считать такой, что ее $(n-1)$-мерный остов пересекает $X$ по множеству размерности $<k$. Это множество ручное в силу леммы о гиперплоских подмножествах. И в силу индукционного предположения мы можем заключить, что оно $Y$-пренебрежимо. Тогда образ $l(X)$ будет объединением конечного числа динамически $Y$-пренебрежимых компактов (образов пересечений $X$ с $n$-мерными симплексами триангулящии), пересечения которых с объединениями остальных являются $Y$-пренебрежимьми. Поэтому аддиционная лемма позволяет нам утверждать, что $l(Y)$ динамически $Y$-пренебрежим. Тогда $l$ не может устойчиво пересекать никакого отображения из $Y$ в $\mathbb{R}^{n}$. Тогда и $f$ не может $\varepsilon$-устойчиво пересекать никакого отображения из $\mathfrak{F}\left(Y, \mathbb{R}^{n}\right)$. А ввиду произвольности $\varepsilon$ отсюда получаем отсутствие устойчивых пересечений компактов $X$ и $Y$ в $\mathbb{R}^{n}$. Теперь на основании теоремы об устойчивых пересечениях получаем $\operatorname{dim} X \times Y<n$ и на основании критерия Дранишникова получаем $Y$-пренебрежимость компакта $X$. Это доказьвает лемму.

ЗАмечАниЕ. Доказанные вьше аддиционная лемма и лемма о динамической пренебрежимости верны и для поликомпактов. Доказательство не меняется.

Для компакта $X$ коразмерности 2 , являюшегося динамически $Y$-пренебрежимым, мы на основании тех же рассуждений можем доказать неравенство $\operatorname{dim} X \times Y<n$, но не можем заключить отсюда его $Y$-пренебрежимость. Таким образом, справедливо также следующее утверждение.

ТЕОРЕМА О ДИНАМИЧЕСКОЙ НЕПРЕНЕБРЕЖИМОСТИ. Ecли компакт $X \subset \mathbb{R}^{n} u$ поликомпакт У длины $m$ имеют размерности $\leqslant n-2 u \operatorname{dim}\left(X \times \prod \mathscr{Y}\right) \geqslant m n$, то $X$ не является динамически $Y$-пренебрежимым.

ЛЕмма О СДВИГЕ. Пусть компакты $X_{1}, \ldots, X_{k} \subset \mathbb{R}^{n}$ пересекаются по мно-

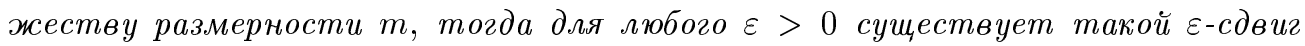
$h: \mathbb{R}^{n} \rightarrow \mathbb{R}^{n}$, чmo $\operatorname{dim}\left(h\left(X_{1}\right) \cap \cdots \cap h\left(X_{k}\right)\right) \leqslant m$.

ДокАЗАТЕльство. Пусть $\omega$ - какое-то $\varepsilon$-покрытие пересечения $\bigcap X_{i}$ открытыми в $\mathbb{R}^{n}$ множествами кратности $\leqslant m+1$. Объединение элементов покрытия обозначим $U$. Пусть $\delta<\varepsilon-$ какое-то положительное число, меньшее всех диаметров множеств из $\bigcup X_{i} \backslash U$, пересекаюших все $X_{i}$. Пусть $\alpha$ - такое покрытие множества $\bigcup X_{i} \backslash U$ открытыми в $\mathbb{R}^{n}$ множествами, что максимальньй диаметр составляющих его множеств меньше чем $\delta / 6$ и все его элементы пересекают $\bigcup X_{i} \backslash U$. Тогда $\beta=\alpha \cup \omega$ является покрытием для $\bigcup X_{i}$. Выбрав в каждом $\beta$ по точке, рассмотрим барищентрическое отображение $g: \bigcup X_{i} \rightarrow \mathbb{R}^{n}$, переводящее точку $x \in \bigcup X_{i}$ в центр тяжести системы выбранных точек с весами, равными расстоянию от $x$ до дополнения, соответствующего выбранной точке, элемента покрытия $\beta$. Тогда $g$ и является искомым $\varepsilon$-сдвигом. Действительно, пусть множество $A$ пересекает все $X_{i}$ и переходит при $g$ в некоторую точку. Тогда $A$ обязательно содержит точку, не покрытую $\alpha$. Действительно, в противном случае для всякого $a \in A$ найдется $V \in \alpha$ такой, что $a \in V$. А так как $V$ пересекает $\bigcup X_{i} \backslash U$, то найдется $a^{\prime} \in \bigcup X_{i} \backslash U$, которая $\delta / 3$-близка к $a$. Совокупность таких точек составляет множество $A^{\prime}$ диаметра меньше чем $\operatorname{diam} A+\delta / 3$. Но множество $A$ имеет диаметр, не превосходящий $\delta / 3$, ибо переходит в точку посредством $\delta / 6$-сдвига. Таким образом, получаем, что $A^{\prime}$ имеет диаметр $<\delta$, не пересекает $U$ и пересекает все $X_{i}$, что противоречит выбору $\delta$. 
Итак, имеется $a \in A$, не покрытая элементами $\alpha$. Но тогда ее образ, совпадающий с $g(A)$, принадлежит образу нерва покрытия $\omega$. То есть мы доказали, что $\bigcap g\left(X_{i}\right)$ содержится в образе нерва покрытия $\omega$, и потому имеет размерность $\leqslant m$, что и требовалось доказать.

Определим устойчивую размерность пересечения отображения $F: \mathscr{X} \rightarrow \mathbb{R}^{n} \mathrm{c}$ множеством $Y$, обозначаемую $\operatorname{sim} Y \cap F$, как наименьшее целое $m$, для которого при любом $\varepsilon>0$ найдутся такое $\varepsilon$-близкое к $F$ отображение $G$ и такой $\varepsilon$-сдвиг $g$ компакта $Y$, что $\operatorname{dim} g(Y) \cap G^{\cap}(\mathscr{X}) \leqslant m$.

ТЕОРЕМА ОБ УСТОЙЧИВОЙ НИЖНЕЙ ОЦЕНКЕ. Пусть $Y$ является компактным подмножеством $\mathbb{R}^{n}$ размерности $\operatorname{dim} Y<n$ и $\mathscr{X}$ такой поликомпакт размерности $<n-1$ и длинь $m$, что $N=\operatorname{dim}\left(Y \times \prod \mathscr{X}\right) \geqslant m n$. Тогда множество отображений $F: \mathscr{X} \rightarrow \mathbb{R}^{n}$, для которьх $\operatorname{sdim} Y \cap F \geqslant N-$ mn, имеет непустую внутренность.

ДокАЗАТЕЛЬСТво. Пусть $k=(n+1) m-N$ и $B^{k}-$ шар размерности $k$. Тогда $0 \leqslant k \leqslant n$ в силу имеющихся у нас ограничений. Составной поликомпакт $Y, \mathscr{X}, B^{k}$ в таком случае имеет размерность произведения составляющих его компактов, равную $(n+1) m$, и согласно теореме об устойчивых пересечениях имеет в $\mathbb{R}^{n}$ устойчивые пересечения. Отсюда согласно теореме о динамической непренебрежимости получаем, что $Y$ не является динамически пренебрежимым составным поликомпактом $B^{k}, \mathscr{X}$. Следовательно, найдутся такое отображение $F: \mathscr{X} \rightarrow \mathbb{R}^{n}$ и такое отображение $g: B^{k} \rightarrow \mathbb{R}^{n}$, что составное отображение $i, g, F: Y, B^{k}, \mathscr{X} \rightarrow \mathbb{R}^{n}$ устойчиво пересекается. Здесь через $i$ обозначено тождественное вложение компакта $Y$ в $\mathbb{R}^{n}$. Покажем, что тогда $\operatorname{sim} Y \cap F \geqslant n-k$. Предположим, что имеется $\varepsilon / 2$-близкое к $F$ отображение $F^{\prime}$, для которого $\operatorname{dim} Y \cap F^{\prime \cap}(\mathscr{X})<n-k$. В силу леммы о сдвиге найдется $\varepsilon / 2$-сдвиг $h$ объединения образов составляюших $F^{\prime}$ отображений $f_{i}^{\prime}$ и компакта $Y$ такой, что пересеченньй образ отображения $h F^{\prime}=\left\{h f_{i}^{\prime}\right\}$ пересекает $h(Y)$ по подмножеству полиэдра размерности $<n-k$. Но тогда мы можем заменить отображение шара $B^{k}$ на $\varepsilon$-близкое и не пересекающее $h G^{\cap}(\mathscr{X}) \cap h(Y)$ отображение $g^{\prime}$. В результате получаем, что составное отображение $h, g^{\prime}, h F^{\prime}$ является $\varepsilon$-близким к $i, g, F$ и имеет пустой пересеченный образ. Ввиду произвольности $\varepsilon$ это противоречит предположению об устойчивости пересечения $i, g, F$. Таким образом, доказано, что $\operatorname{sdim} Y \cap F \geqslant n-k$. Вспоминая теперь, что $k=(n+1) m-N$, получаем требуемое неравенство. Теорема доказана.

Будем говорить, что компакт $X$ имеет устойчивый размерностный тип, если найдется такое $\varepsilon>0$, что для всякого $\varepsilon$-отображения $f: X \rightarrow Y$ будет $\operatorname{Dim} Y \geqslant$ $\operatorname{Dim} X$. Следующее утверждение автору доказать не удалось:

ГИПОТЕЗА. Если компакты $X, Y$, коразмерности 2, имеют устойчивый размерностный тип, то множество пар отображений $f, g: X, Y \rightarrow \mathbb{R}^{n}$, для котоpых $\operatorname{Dim} f(X) \cap g(Y) \geqslant \operatorname{Dim}(X \times Y)\langle-\rangle$, имеет непустую внутренность. 


\section{СПИСОК ЛИТЕРАТУРЫ}

[1] Урысон П.С. Труды по топологии и другим областям математики. М.: Гостехиздат, 1951.

[2] Александров П. С., Пасынков Б.А. Введение в теорию размерности. М.: Наука, 1973.

[3] Гуревич В., Волмен Г. Теория размерности. М.: ИЛ, 1948.

[4] Alexandroff P.S. Dimensionstheorie. Ein Beitrag zur Geometrie der abgeschlossenen Mengen // Math. Ann. 1932. V. 106. P. 161-238.

[5] Понтрягин Л.С. О моих работах по топологии и топологической алгебре // Труды МИАН. 1984. Т. 168. С. 236-249.

[6] Pontryagin L.S. Sur une hypothèse fondamentale de la dimension // C. R. Acad. Sci. 1930. V. 190. P. 1105-1107.

[7] Dranishnikov A. N., Repovs D., Shchepin E. V. On approximation and embedding problems for cohomological dimension // Topol. Appl. 1994. V. 55. № 1. P. 67-86.

[8] Болтянский В. Г. Пример двумерного компакта, топологический квадрат которого имеет размерность, равную трем // Докл. АН СССР. 1949. Т. 67. № 4. С. 597-599.

[9] Болтянский В.Г. О размерной полноценности компактов // Докл. АН СССР. 1949. T. 67. № 5. C. 773-777.

[10] Бокштейн М. Ф. О гомологических инвариантах топологических пространств, I // Труды МMO. 1956. Т. 5. С. 3-80.

[11] Бокштейн М. Ф. О гомологических инвариантах топологических пространств, II // Труды ММО. 1957. Т. 6. С. 3-133.

[12] Kodama Y. On a problem of Alexandroff concerning the dimension of product spaces, I // J. Math. Soc. Japan. 1958. V. 10. P. 380-404.

[13] Kodama Y. Test spaces for homological dimension // Duke Math. J. 1962. V. 29. P. 41-50.

[14] Кузьминов В.И. Гомологическая теория размерности // УМН. 1968. Т. 23. № 5. C. $3-49$.

[15] Дранишников А. Н. О реализации размерных функций. II // Сиб. матем. журн. 1988. T. 29. № 1. C. $32-38$.

[16] Дранишников А. Н. Гомологическая теория размерности // УМН. 1988. Т. 43. № 4. C. $11-55$.

[17] McCullough D., Rubin L. R. Some $m$-dimensional compacta admitting a dense set of imbeddings into $\mathbb{R}^{2 m} / /$ Fund. Math. 1989. V. 133. № 3. P. 237-245.

[18] Spiez S., Toruńczyk H. Moving compacta in $\mathbb{R}^{n}$ apart // Topol. Appl. 1991. V. 41. № 3. P. 193-204.

[19] Dranishnikov A. N., Repovs D., Shchepin E. V. Transversal intersection formula // Topol. Appl. 1998. V. 85. P. 93-117.

[20] Dranishnikov A.N. On the mapping intersection problem // Pacific J. Math. 1996. V. 173. № 2. P. 403-412.

[21] Дранишников А. Н. Продолжение отображений в CW комплексы // Матем. сб. 1991. T. 182. №9. C. 1300-1310.

[22] Dranishnikov A. N., Repovs D., Shchepin E. V. On the failure of the UrysohnMenger sum formula for cohomological dimension // Proc. Amer. Math. Soc. 1994. V. 120. P. $1267-1270$.

[23] Rubin L. R. Characterizing cohomological dimension: The cohomological dimension of $A \cup B$ // Topol. Appl. 1991. V. 40. № 3. P. 233-263.

[24] Dydak J. Cohomological dimension of metrizable spaces // Trans. Amer. Math. Soc. 1993. V. 337. № 1. P. 219-234.

[25] Dydak J. Cohomological dimension of metrizable spaces, II // Trans. Amer. Math. Soc. 1996. V. 348. № 4. P. 1647-1661.

[26] Olszewski W. Completion theorem for cohomological dimensions // Proc. Amer. Math. Soc. 1995. V. 123. P. 2261-2264.

[27] Александров П. С. Гомологическая теория размерности. М.: Наука, 1971.

[28] Скляренко Е. Г.О зацеплении циклов // УМН. 1966. Т. 21. № 4. С. 77-90. 
[29] Dranishnikov A. N., Dydak J. Extension dimension and extension types // Труды МИАН. 1996. Т. 212. С. 61-94.

[30] Dranishnikov A. N., Repovs D. Cohomological dimension with respect to perfect groups // Topol. Appl. 1996. V. 20. P. 1-18.

[31] Dydak J., Yokoi K. Hereditarily aspherical compacta // Proc. Amer. Math. Soc. 1996. V. 124. №6. P. 1933-1940.

[32] Постников М. М. Локализация топологических пространств // УМН. 1977. Т. 32. №6. C. $117-181$.

[33] Дранишников А.Н. О теории продолжения отображений компактов // УМН. 1998. № 5. C. $65-72$.

[34] Dranishnikov A. N. On the dimension of the product of two compacta and the dimension of their intersection in general position in Euclidean space // Trans. Amer. Math. Soc. (to appear)

[35] Фукс Л. Бесконечные абелевы группы. Т. 1. М.: Мир, 1974.

[36] Walsh J. J. Dimension, cohomological dimension, and cell-like mappings // Lecture Notes in Math. 1981. V. 870. P. 105-118.

[37] Дранишников А.Н. Теорема Эйленберга-Борсука для отображений в произвольньй комплекс // Матем. сб. 1994. Т. 185. № 4. С. 81-90.

[38] Сулливан Д. Геометрическая топология. М.: Мир, 1975.

[39] Dold A., Thom R. Quasifaserungen und unendliche symmetrische Produkte // Ann. of Math. 1958. V. 67. P. 239-281.

[40] Dranishnikov A., Dydak J. Extension theory of separable metrizable spaces with applications to dimension theory // Preprint, 1997.

[41] Dranishnikov A. N., Uspenskij V. V. Light maps and extensional dimension // Topol. Appl. 1997. V. 80. №1-2. P. 91-99.

[42] Chogoshvili G.S. On a theorem in the theory of dimensionality // Compositio Math. 1937. V. 5. P. 292-298.

[43] Dranishnikov A. N. On Chogoshvili's conjecture // Proc. Amer. Math. Soc. 1997. V. 125. № 7. P. $2155-2160$.

[44] Frankl F. Charakterisierung der $(n-1) r$-dimensionalen abgeschlossenen Mengen des $R^{n}$ // Math. Ann. 1930. V. 103. P. 784-787.

[45] Штанько М. А. Вложения компактов в эвклидово пространство // Матем. сб. 1970. T. 83. № 2. C. 234-255. 